\title{
The role of humoral autoimmunity in gastrointestinal neuromuscular diseases
}

\author{
Andrew William Hubball \\ A thesis submitted for the degree of Doctor of Philosophy at the University of London
}

August 2010

The Wingate Institute of Neurogastroenterology

Blizard Institute of Cell and Molecular Science

Barts and The London School of Medicine and Dentistry

Queen Mary, University of London 
To Carlton 


\section{ABSTRACT}

Dysfunction of the gastrointestinal neuromuscular apparatus (including interstitial cells of Cajal) is presumed to underlie a heterogeneous group of disorders collectively termed gastrointestinal neuromuscular diseases (GINMDs). Humoral (antibody)-mediated autoimmunity directed against ligand- or voltage-gated ion channels or associated proteins involved in neuromuscular transmission is associated with several acquired neuromuscular diseases of the periphery and is now implicated in an increasing number of less well-characterised diseases. Anti-channel antibodies have been reported in small numbers of patients with GINMD, particularly in those with GI dysfunction secondary to an underlying disease such as neoplasia or infection. However, little is known of humoral autoimmunity in primary GINMDs.

This thesis investigated the association between anti-channel antibodies and GINMD, and the human GI tract as a potential target of anti-channel antibodies. The presence of antivoltage- and ligand-gated antibodies was investigated in the serum of patients with primary achalasia, enteric dysmotility and intestinal pseudo-obstruction, and in those with GINMD secondary to Chagas' disease. The functional effect of sera from some of these patients on colonic smooth muscle contractility was also characterised. Finally, the distribution of six voltage-gated potassium channels (VGKCs), which serve essential roles in the peripheral and central nervous systems and are known targets of pathological autoantibodies, were investigated in all layers of the human GI tract.

Our findings suggest that currently recognised anti-channel antibodies are not a common pathogenic factor in primary GINMDs. Anti-channel antibodies, in particular anti-VGKC antibodies, were however found in a significant number of individuals with Chagas' disease. Furthermore, circulating factors in patients with chagasic GI disease altered GI smooth muscle contractility in vitro which may have pathological relevance to GI 
dysfunction. VGKCs were found throughout the human GI tract, especially in enteric neurons and epithelial cells, which has significance for both normal function and in relation to the GI tract as a target organ for anti-channel autoimmunity. 


\section{ACKNOWLEDGEMENTS}

I would like to thank my supervisors, Mr Charles Knowles and Professor Joanne Martin, for their support and kindness throughout my studies. I would also like to thank Professor Jeremy Powell-Tuck for his contribution to this work, especially in the early stages. I am extremely grateful to the Pseudo-obstruction Research Trust (PORT) and the Harold Hyam Wingate Foundation for their financial support, without which much of this research would not have been possible. Thank you to Christopher Evagora and the staff of the BICMS Pathology Lab for assisting with the immunohistochemical procedures. My thanks also go to Dr Bethan Lang and Professor Angela Vincent at the John Radcliffe Hospital, Oxford, who welcomed me warmly into their department and taught me a great deal about autoimmune channelopathies. Thank you also to Professor Ricardo Brandt de Oliveria, and Dr Miguel Souza at the Departamento de Clinica Medica Faculdade de Medicina, University of Sao Paulo, for collection of sera from patients with Chagas' disease, and to Professor Roberto De Giorgio at St. Orsola-Malpighi Hospital, Bologna, Italy, for his continuing efforts examining the functional significance of our findings. Finally, I would like to thank all the brilliant friends I have made during this time. 


\section{PUBLICATIONS AND ABSTRACTS}

\section{Papers}

A Hubball, JE Martin, B Lang, R De Giorgio, CH Knowles. The role of humoral autoimmunity in gastrointestinal neuromuscular diseases. Progress in Neurobiology 2009;87(1):10-20

\section{Abstracts}

A Hubball, R Patel, M Baker, J Powell-Tuck, CH Knowles, and JE Martin. KV1 voltagegated potassium channels in the gastrointestinal tract: Distribution and potential mediators of motility dysfunction. Gut 2007;56(suppl. 2):a195

A. Hubball, R Patel, D Valliani, M Baker, J Powell-Tuck, CH Knowles, JE Martin. The Distribution of $\mathrm{K}_{\mathrm{V}} 1$ Voltage-gated Potassium Channel Subunits in the Human Gastrointestinal Tract. Journal of Pathology. 2007; 213 (suupl.1): 35

A Hubball, $\mathrm{CH}$ Knowles, JE Martin. $\mathrm{K}_{\mathrm{V}} 1$ voltage-gated potassium channels in the gastrointestinal tract: distribution and potential mediators of motility dysfunction. Neurogastroenterology and Motility. 2008; 20 (suppl 1): 51 


\section{CONTENTS}

Title page

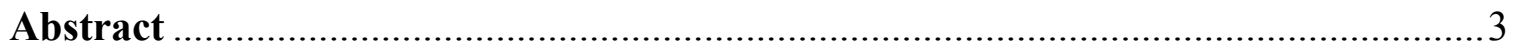

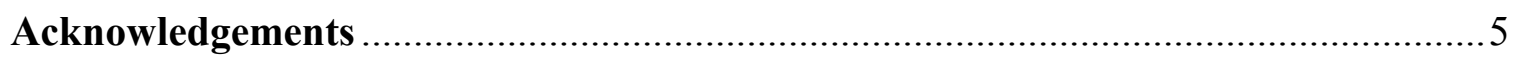

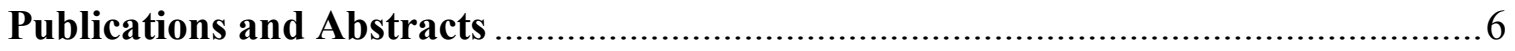

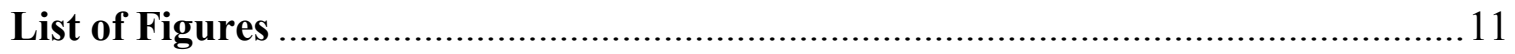

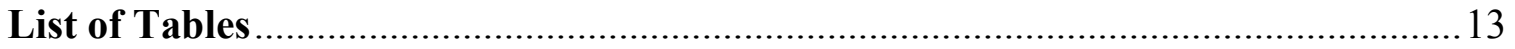

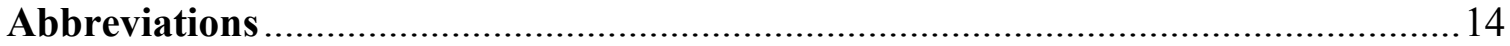

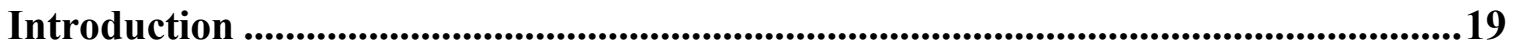

1.1 Gastrointestinal neuromuscular diseases ....................................................... 19

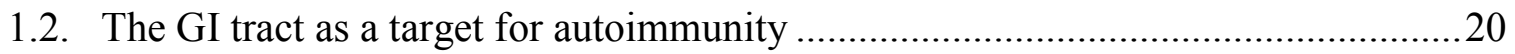

1.2.1 Neuronal ion channels of the GI tract..............................................21

1.3. 'Classical' antibody-mediated neurological diseases .......................................26

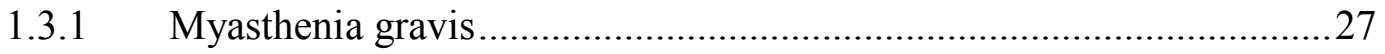

1.3.2 Lambert Eaton myasthenic syndrome ….......................................27

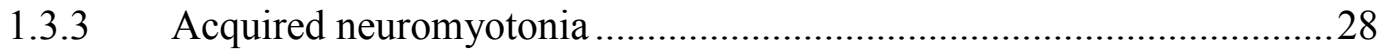

1.4 Evidence for antibody mediation of autoimmune diseases .................................29

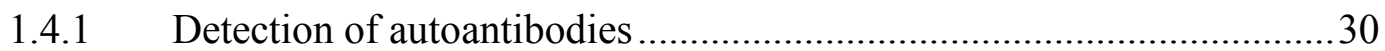

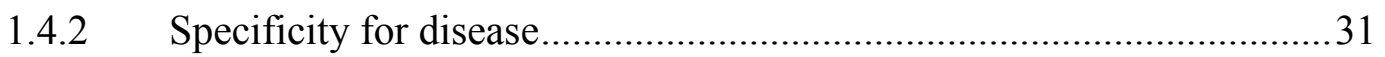

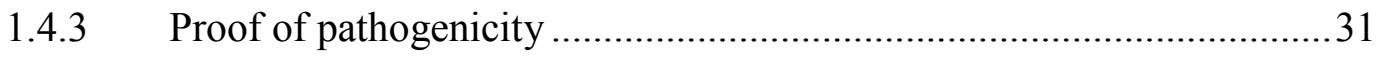

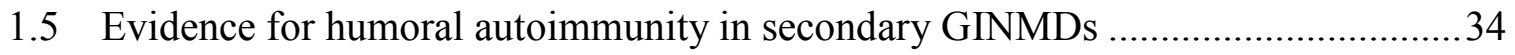

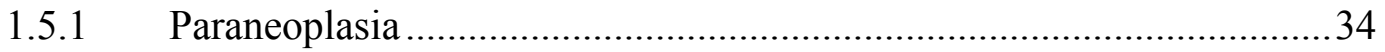

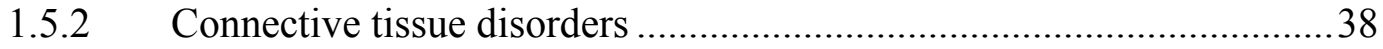

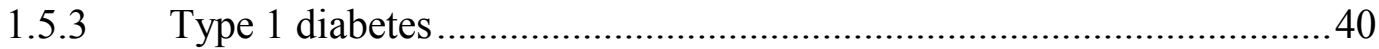

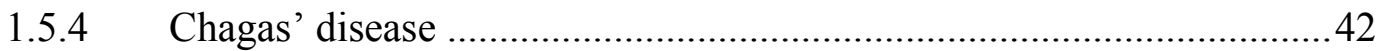


1.6 Evidence for humoral autoimmunity in primary GINMDs

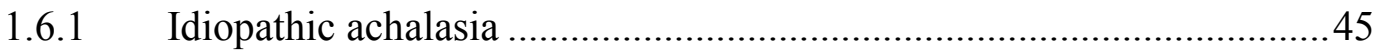

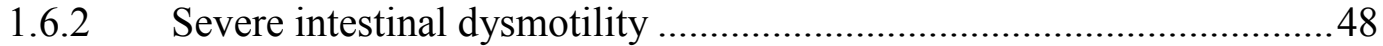

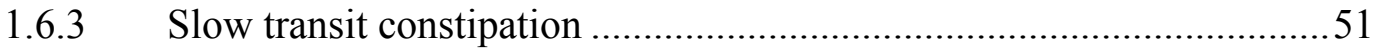

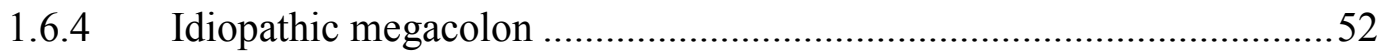

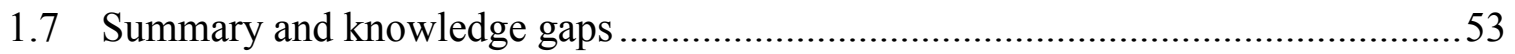

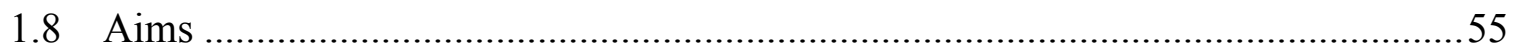

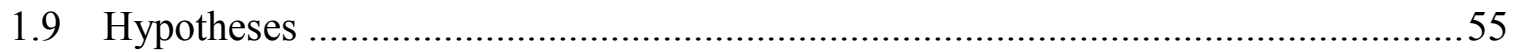

Materials and Methods ...............................................................................................56

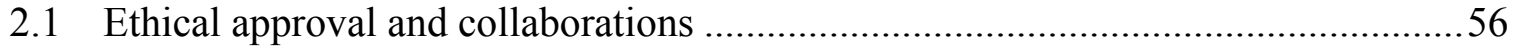

2.2 Detection of autoantibodies in patients with GINMDs ..................................5

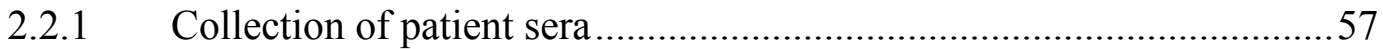

2.2.2 Anti-neuronal autoantibody immunofluorescent assay .......................6 68

2.2.3 Anti-channel antibody radioimmunoprecipitation assay.....................69

2.2.4 Anti-M $\mathrm{M}_{2}$ muscarinic cell-based assay .......................................... 73

2.3 Functional in vitro effects of chagasic sera .................................................. 79

2.3.1 In vitro contractility experiments ................................................... 79

2.4 Expression and localisation of enteric $\mathrm{K}_{V} 1$ channels in the human GI tract ...........82

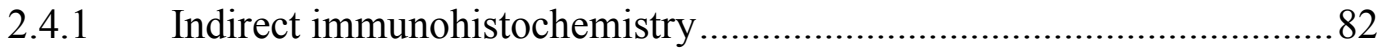

2.4.2 Characterisation of enteric neuronal $\mathrm{K}_{\mathrm{V}} 1.4$ localisation .....................8 85

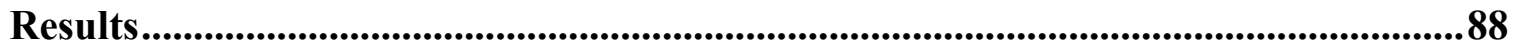

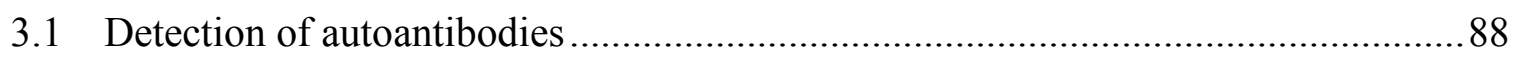

3.1.1 Anti-neuronal antibody assay ................................................. 88

3.1.2 Anti-channel antibody radioimmunoprecipitation assays ....................90

3.1.3 Anti-M $\mathrm{M}_{2}$ muscarinic acetylcholine receptor cell-based assay ............... 95 


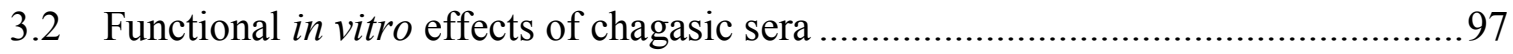

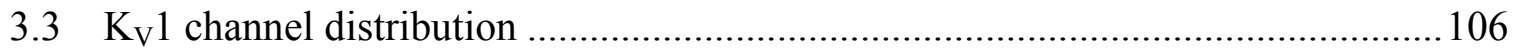

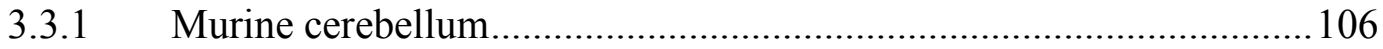

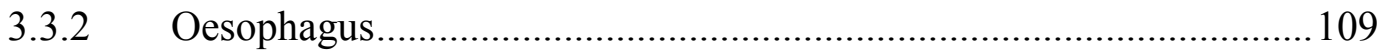

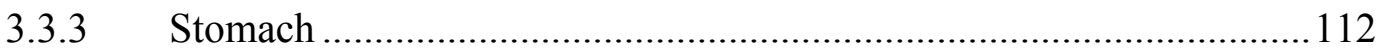

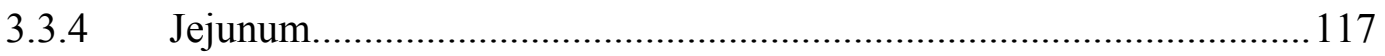

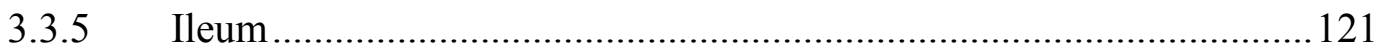

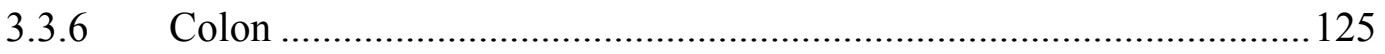

3.3.7 $\quad \mathrm{K}_{\mathrm{V}} 1.4$ localisation in human gastric myenteric plexus.........................129

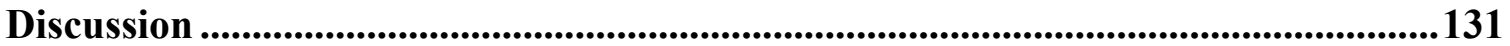

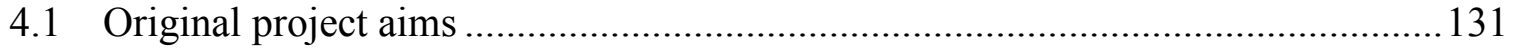

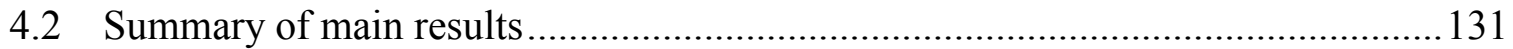

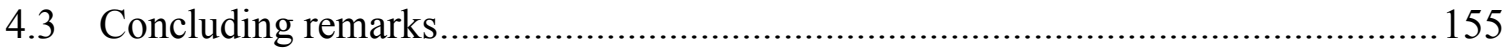

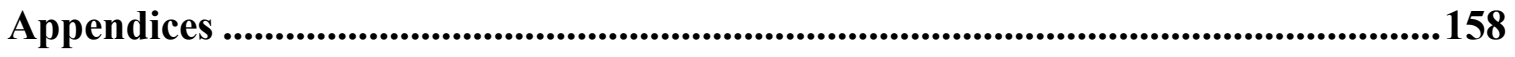

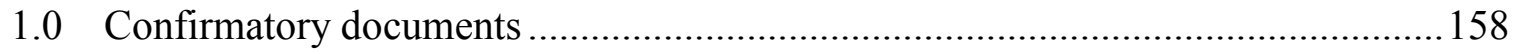

2.0 Anti-neuronal immunofluorescent assay ............................................................. 164

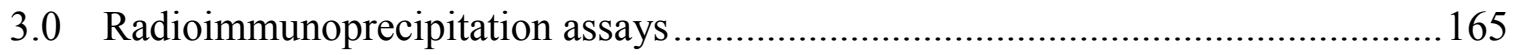

3.1 Preparation of channel extracts ............................................................. 165

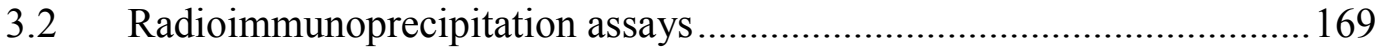

3.3 Conversion of observed counts to concentrations ..................................173

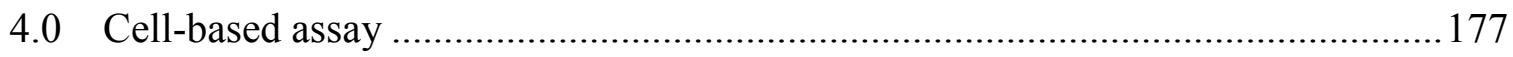

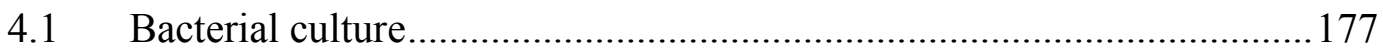

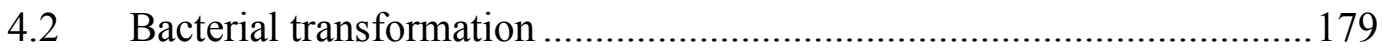

4.3 DNA isolation and sequencing ............................................................ 180

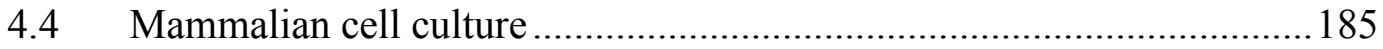




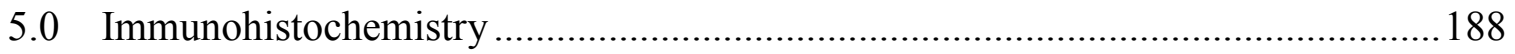

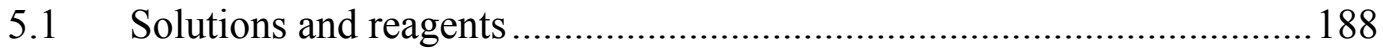

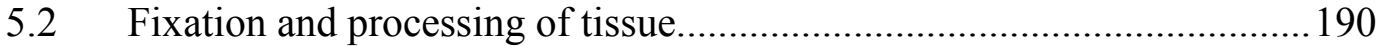

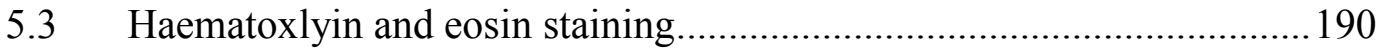

$5.4 \quad$ Indirect-immunohistochemical staining protocol ............................... 191

5.5 Indirect-immunofluorescent staining protocol .................................. 192

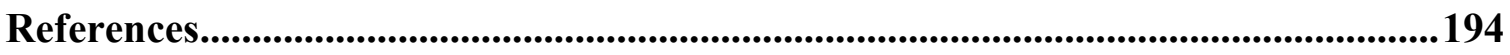




\section{LIST OF FIGURES}

Figure 1.1: Phylogenetic tree for the voltage-gated potassium channel family.......................24

Figure 1.2: Pro-apoptotic effects of anti-Hu antibody on cultured enteric neurons.....................37

Figure 2.1: Oesophageal manometry tracing showing a normal swallow...........................59

Figure 2.2: $\quad$ Oesophageal manometry tracing from a patient with achalasia......................60

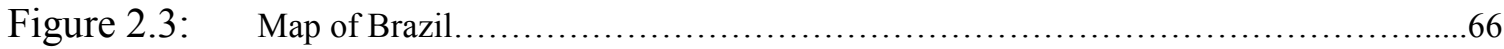

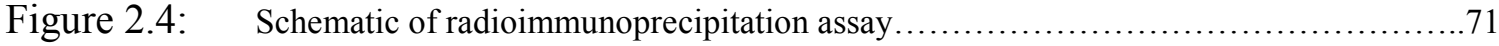

Figure 2.5: Organ bath for electrical field stimulation and measurement of smooth

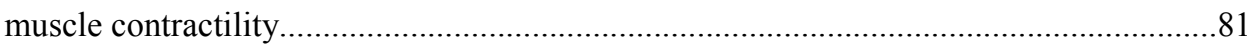

Figure 3.1: Cerebellar anti-neuronal immunostaining ....................................... 89

Figure 3.2: $\quad$ Scatter plot of results from anti- $\alpha 3$ nAChR immunoprecipitation assay...................91

Figure 3.3: $\quad$ Scatter plot of results from anti-GAD immunoprecipitation assay......................92

Figure 3.4: Scatter plot of results from anti-VGCC immunoprecipitation assay....................93

Figure 3.5: Scatter plot of results from anti-VGKC immunoprecipitation assay......................94

Figure 3.6: Indirect immunofluorescent staining of transfected HEK293 cells with

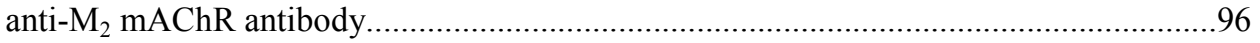

Figure 3.7: Indirect immunofluorescent staining of transfected HEK293 cells with chagasic sera.....97

Figure 3.8: Human colonic smooth muscle responses to EFS and pharmacological

modulation of responses.

Figure 3.9: Effects of serum from patients with Chagas' colon disease on human colonic smooth muscle responses to EFS.

Figure 3.10: Effects of serum from Chagas' disease and healthy controls patients on human colonic smooth muscle responses to EFS

Figure 3.11: Serum effects on human colonic smooth muscle EFS responses in the presence of atropine and L-NAME

Figure 3.12: Serum effects on human colonic baseline muscle tension in the presence of atropine and L-NAME.

Figure 3.13: Negative immunostaining control of mouse cerebellum. 107 
Figure 3.14: Anti-K $\mathrm{K}_{\mathrm{V}} 1$ immunostaining of mouse cerebellum................................. 108

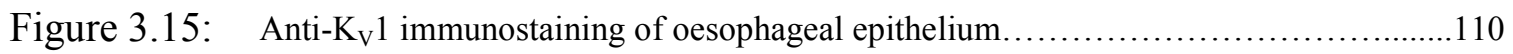

Figure 3.16: Anti- $\mathrm{K}_{\mathrm{V}} 1$ immunostaining of human oesophageal myenteric ganglia..............................111

Figure 3.17: Anti- $\mathrm{K}_{\mathrm{V}} 1.5$ immunostaining of human oesophageal smooth muscle................................112

Figure 3.18: $\quad \mathrm{K}_{\mathrm{V}} 1$ immunostaining of human gastric epithelium........................................................114

Figure 3.19: $\mathrm{K}_{\mathrm{V}} 1$ immunostaining of human gastric submucosal neurons..........................................115

Figure 3.20: $\quad \mathrm{K}_{\mathrm{V}} 1$ immunostaining of human gastric myenteric ganglia...........................................116

Figure 3.21: $\mathrm{K}_{\mathrm{V}} 1$ immunostaining of human jejunal mucosa..........................................................118

Figure 3.22: $\quad \mathrm{K}_{\mathrm{V}} 1$ immunostaining of human jejunal submucosal ganglia...........................................119

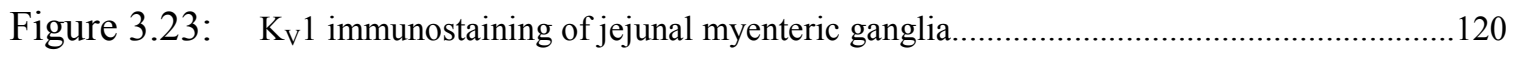

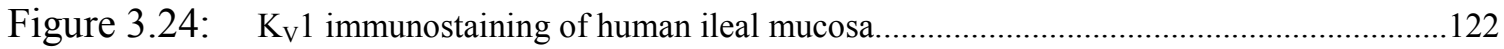

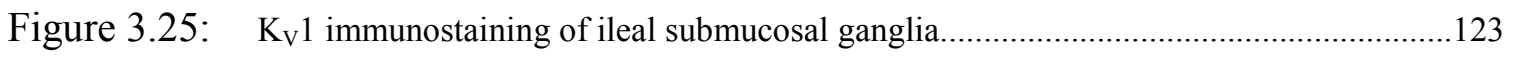

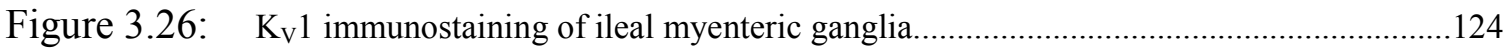

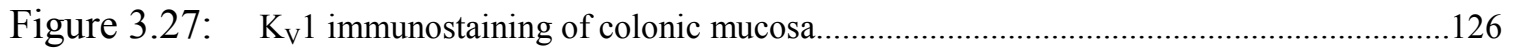

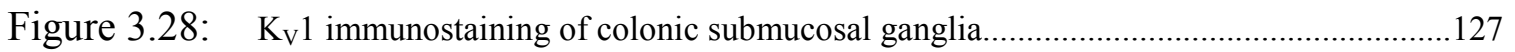

Figure 3.29: $\quad \mathrm{K}_{\mathrm{V}} 1$ immunostaining of colonic myenteric ganglia.......................................................128

Figure 3.30: Co-localisation of $\mathrm{K}_{\mathrm{V}} 1.4$ and neuronal markers in the gastric myenteric plexus............130

Figure 4.1: Geographical distributions of T.cruzi lineages (Tc I - VI) ............................................140 


\section{LIST OF TABLES}

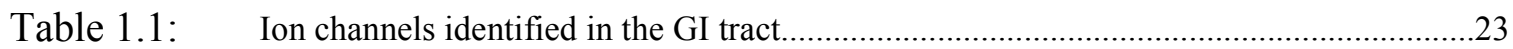

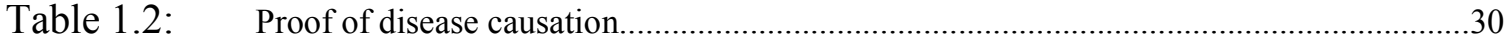

Table 1.3: Evidence for humoral autoimmunity in AINMD and GINMD.........................................54

Table 2.1: $\quad$ Symptoms of severe intestinal dysmotility .....................................................61

Table 2.2: Investigation findings in patients with SID $\quad$................................................................62

Table 2.3: Histopathological findings in full-thickness jejunal biopsies from patients with SID......63

Table 2.4: Disease-subgroups of Chagas' disease patients..................................................................65

Table 2.5: $\quad$ Basic clinical data and demographics for patients included in assay studies....................67

Table 2.6: Anti- $\mathrm{K}_{\mathrm{V}} 1$ channel antibody dilutions used for indirect immunohistochemistry.................85

Table 2.7: Antibodies used in indirect immunofluorescence..........................................................

Table 3.1: Subjects investigated for sera effects on colonic smooth muscle contractility..................96 


\begin{tabular}{|c|c|}
\hline \multicolumn{2}{|c|}{ ABBREVIATIONS } \\
\hline $\mathrm{ACh}$ & acetylcholine \\
\hline AGID & autoimmune gastrointestinal dysmotility \\
\hline AINMD & autoimmune neuromuscular disease \\
\hline ANA & anti-neuronal autoantibody \\
\hline ANNA-1 & anti-neuronal nuclear antibody type-1 \\
\hline ANNA-2 & anti-neuronal nuclear antibody type- 2 \\
\hline BICMS & Blizzard Institute of Cell and Molecular Science \\
\hline BSA & bovine serum albumin \\
\hline cAMP & cyclic adenosine monophosphate \\
\hline cGMP & cyclic guanosine monophosphate \\
\hline${ }^{\circ} \mathrm{C}$ & degrees Celsius \\
\hline cDNA & deoxyribonucleic acid \\
\hline ChAT & choline acetyl transferase \\
\hline CHRM2 & chromosome 2 \\
\hline $\mathrm{CI}$ & confidence interval \\
\hline CIPO & chronic intestinal pseudo-obstruction \\
\hline $\mathrm{cm}^{2}$ & centimetres squared \\
\hline $\mathrm{CmTx}$ & conotoxin MVIIC \\
\hline CNS & central nervous system \\
\hline $\mathrm{COX}-2$ & cyclo-oxygenase-2 \\
\hline $\mathrm{CO}_{2}$ & carbon dioxide \\
\hline DAB & 3,3'-Diaminobenzidine \\
\hline DAPI & 4',6-diamidino-2-phenylindole \\
\hline D-IBS & diarrhoea-predominant irritable bowel syndrome \\
\hline DMEM & Dulbecco’s Modified Eagles Medium \\
\hline DNA & deoxyribonucleic acid \\
\hline DnTx & dendrotoxin \\
\hline
\end{tabular}




$\begin{array}{ll}\text { ECG } & \text { electrocardiograph } \\ \text { EcoRI } & \text { Escherichia coli restriction enzyme one } \\ \text { E.coli } & \text { Escherichia coli } \\ \text { ED } & \text { enteric dysmotility } \\ \text { EFS } & \text { electrical field stimulation } \\ \text { ELISA } & \text { enzyme-linked immunoadsorbent assay } \\ \text { ENS } & \text { enteric nervous system } \\ \text { Epb } & \text { epibatidine } \\ \text { ERG } & \text { ether-a-go-go } \\ \text { FCS } & \text { fetal calf serum } \\ \text { fM } & \text { femtomole } \\ \text { g } & \text { gram } \\ \text { GAD } & \text { glutamic acid decarboxylase } \\ \text { GFP } & \text { green fluorescent protein } \\ \text { GI } & \text { gastrointestinal } \\ \text { GINMD } & \text { gastrointestinal neuromuscular disease } \\ \text { GORD } & \text { gastro-oesophageal reflux disease } \\ \text { h } & \text { hours } \\ \mathrm{H}_{2} \mathrm{O}_{2} & \text { hydrogen peroxide } \\ \mathrm{H \& E} & \text { intestinal epithelial cell line } 6 \\ \mathrm{HEK} & \text { haematoxylin and eosin } \\ \mathrm{HEPES} & \text { human embryonic kidney } \\ \mathrm{HLA} & \text { 4-(2-hydroxyethyl)-1-piperazineethanesulfonic acid } \\ \mathrm{Hz} & \text { human leucocyte antigen } \\ \mathrm{I}_{\mathrm{A}} & \text { irritable bowel syndrome } \\ \text { IBS } & \text { iCC } \\ \text { IEC-6 } & \end{array}$




\begin{tabular}{|c|c|}
\hline $\operatorname{IgG}$ & immunoglobulin $\mathrm{G}$ \\
\hline $\mathrm{I}_{\mathrm{K}}$ & potassium current \\
\hline IMS & industrial methylated spirit \\
\hline iNOS & inducible nitric oxide synthase \\
\hline $\mathrm{K}$ & potassium \\
\hline $\mathrm{K}_{\mathrm{Ca}}$ & calcium-activated potassium (channel) \\
\hline $\mathrm{KCl}$ & potassium chloride \\
\hline $\mathrm{KH}_{2} \mathrm{PO}_{4}$ & monopotassium phosphate \\
\hline $\mathrm{K}_{\mathrm{V}}$ & voltage-gated potassium (channel) \\
\hline $\mathrm{K}_{\mathrm{V}} 1$ & group 1 voltage-gated potassium (channel) \\
\hline $\mathrm{L}$ & litre \\
\hline LB & lysogeny broth \\
\hline LEMS & Lambert-Eaton myasthenia syndrome \\
\hline L-NAME & $\mathrm{N}$ - $\omega$-nitro-L-arginine methyl ester \\
\hline LOS & lower oesophageal sphincter \\
\hline $\mathrm{M}_{2,3}$ & muscarinic receptor 2,3 \\
\hline $\mathrm{mA}$ & milliampere \\
\hline $\mathrm{mAChR}$ & muscarinic acetylcholine receptor \\
\hline MG & myasthenia gravis \\
\hline $\mathrm{MgSO}_{4}$ & magnesium sulphate \\
\hline $\min$ & minute \\
\hline $\mathrm{ml}$ & millilitre \\
\hline $\mathrm{mm}$ & millimetre \\
\hline $\mathrm{mM}$ & millimole \\
\hline mRNA & messenger ribonucleic acid \\
\hline $\mathrm{ms}$ & millisecond \\
\hline MuSK & muscle-specific receptor tyrosine kinase \\
\hline nAChR & nicotinic acetylcholine receptor \\
\hline
\end{tabular}




\begin{tabular}{|c|c|}
\hline $\mathrm{NaCl}$ & sodium chloride \\
\hline $\mathrm{NaHCO}_{3}$ & sodium bicarbonate \\
\hline NF & neurofilament \\
\hline $\mathrm{Ng}$ & nanogram \\
\hline NK & neurokinin \\
\hline $\mathrm{nm}$ & nanometre \\
\hline NMT & neuromyotonia \\
\hline NO & nitric oxide \\
\hline NOS & nitric oxide synthase \\
\hline $\mathrm{O}_{2}$ & oxygen \\
\hline PBS & phosphate buffered saline \\
\hline PCA & Purkinje cell cytoplasmic antibody \\
\hline $\mathrm{PGE}_{2}$ & prostaglandin E2 \\
\hline $\mathrm{pM}$ & picomole \\
\hline PNS & peripheral nervous system \\
\hline PSA & penicillin, amphotericin and streptomycin \\
\hline RNA & ribonucleic acid \\
\hline RT & room temperature \\
\hline $\mathrm{rpm}$ & revolutions per minute \\
\hline s & second \\
\hline SCLC & small-cell lung cancer \\
\hline SID & severe intestinal dysmotility \\
\hline $\mathrm{SSc}$ & systemic sclerosis \\
\hline STC & slow-transit constipation \\
\hline TFB & transformation buffer \\
\hline $\mathrm{T}$ & temperature \\
\hline TTX & tetrodotoxin \\
\hline$\mu$ & micron \\
\hline
\end{tabular}




$\begin{array}{ll}\mu \mathrm{g} & \text { microgram } \\ \mu \mathrm{l} & \text { microlitre } \\ \mathrm{UV} & \text { ultraviolet } \\ \mathrm{V} & \text { volt } \\ \text { VGCC } & \text { voltage-gated calcium channel } \\ \text { VGKC } & \text { voltage-gated potassium channel } \\ \text { WB } & \text { wash buffer } \\ \text { w/v } & \text { weight / volume } \\ \text { XhoI } & \text { Xanthomonas holcicola I }\end{array}$




\section{INTRODUCTION}

\subsection{GASTROINTESTINAL NEUROMUSCULAR DISEASES}

Gastrointestinal neuromuscular diseases (GINMDs) are a clinically heterogeneous group of disorders in which symptoms are presumed or proven to arise as a result of neuromuscular (including interstitial cell of Cajal) dysfunction (Knowles et al. 2010). This classification requires both symptoms of impaired motor activity and demonstrable abnormalities in motor function, for example manometric abnormalities with or without evidence of transient or persistent radiological visceral dilatation (Wingate et al. 2002). These disorders may be due to relatively rare congenital defects, for example Hirschsprung's disease, where the pathophysiologies are to some extent elucidated (Heanue et al. 2007), however most GINMDs are acquired in later life and may be primary or secondary to another established disease.

Primary GINMDs include oesophageal achalasia (Hertz 1915), gastroparesis (Shellito et al. 1984), enteric dysmotility (ED) (Wingate et al. 2002) and chronic idiopathic intestinal pseudo-obstruction (CIPO) (Dudley et al. 1958) (for the purposes of this thesis ED and CIPO are referred to collectively as severe intestinal dysmotility: SID), slow-transit constipation (STC) (Preston et al. 1986) and idiopathic megabowel (Bodian et al. 1949).. Some international consensus exists on the clinico-physiological diagnosis of such disorders (Wingate et al. 2002). Histopathological changes in nerves (Schuffler et al. 
1982), smooth muscle (Smith et al. 1997) and interstitial cells of Cajal (ICC) (Huizinga 1998; He et al. 2000; Farrugia 2008) have been variably described in most and have received recent attention by an international working group (Knowles et al. 2010).

Clinically similar disorders can arise in association with other diseases. Such secondary GINMDs include intestinal pseudo-obstruction occurring as a paraneoplastic syndrome, most commonly in association with small cell lung carcinoma (SCLC) (Schuffler et al. 1983; Chinn et al. 1988; Sodhi et al. 1989; Lucchinetti et al. 1998), but also in cases of bronchial carcinoid (Gerl et al. 1992), thymoma (Pande et al. 1999), ganglioneuroblastoma and neuroblastoma (Schobinger-Clement et al. 1999; Martin et al. 2008) and ovarian carcinoma (Lee et al. 2001). GINMDs may also occur in association with infective systemic diseases such as Chagas' disease (South American trypanosomiasis) (Ferreira-Santos 1961), connective tissue disorders such as scleroderma (Treacy et al. 1963; Cohen et al. 1972; Battle et al. 1981) and some endocrine and metabolic disorders e.g. diabetes mellitus (Werth et al. 1992) and amyloidosis (Legge et al. 1970).

\subsection{THE GI TRACT AS A TARGET FOR AUTOIMMUNITY}

A significant proportion of GI diseases have an inflammatory / immune-mediated aetiology e.g. coeliac and inflammatory bowel disease. Although less studied, there is increasing experimental and clinical evidence that some GINMDs could also be immunemediated. Innate and adaptive immune mechanisms can both modulate neuromuscular function acutely, and lead to changes in sensorimotor function that are sustained after resolution of a discrete inflammatory stimulus including one confined to the mucosa. This evidence, based on extensive study of the Trichinella-infected jejunum of small experimental animals (Collins 1996; Barbara et al. 1997) is supported by several studies in IBS that demonstrate increased numbers of intra-mucosal inflammatory cells including 
mast cells (Barbara et al. 2004; Wang et al. 2004) and T lymphocytes (Spiller et al. 2000; Chadwick et al. 2002), as well as increased levels of pro-inflammatory mediators (Gonsalkorale et al. 2003; O'Mahony et al. 2005). Inflammatory cells have also been shown to infiltrate deeper layers of the muscle wall or the myenteric plexus (hence the terms of leiomyositis and ganglionitis, respectively) and tend to be associated with more severe motor dysfunction. Similar findings have been made in CIPO (Ruuska et al. 2002; Tornblom et al. 2002; Knowles et al. 2004; Haas et al. 2005; Tornblom et al. 2007; Knowles et al. 2008; Lindberg et al. 2009).

Humoral (antibody)-mediated autoimmunity is associated with several well-established acquired neuromuscular diseases (below) and is implicated in an increasing number of less well-characterised disorders (Newsom-Davis et al. 1991), particularly of the central nervous system (Lang et al. 2003). Although less studied, there is some evolving evidence, especially in secondary GINMDs that the enteric neuromusculature could be a target for autoimmune attack. Here the diversity of ion channels and other receptors, and in this regard, potential epitopes, is only currently being realised (Vianna-Jorge et al. 2000; Galligan 2002; Schaufele et al. 2005).

\subsubsection{NEURONAL ION CHANNELS OF THE GI TRACT}

It is likely (based on observations of neurotransmitter heterogeneity and numbers of enteric neurons, akin to the spinal cord (Gershon et al. 1981; Furness 2000) that the profile of ion channels in the gut is similar to that of the central and peripheral nervous systems (CNS and PNS respectively), although less well-studied. To date, a diversity of voltage-gated calcium (Starodub et al. 1999), potassium (Zholos et al. 1999; Hatton et al. 2001) and sodium channels (Franklin et al. 1993), ligand-gated ion channels (Galligan 2002) and G-protein-coupled (North et al. 1985), neurokinin (Portbury et al. 1996), adrenergic (Scheibner et al. 2002) and serotinergic receptors (Pan et al. 1997) have been 
identified in the enteric nervous system (ENS) and play important roles in gastrointestinal sensation and motility (Cervero et al. 2003) (Table 1.1). Of particular relevance to this thesis are voltage-gated potassium channels (VGKCs) which are described in more detail below.

\subsubsection{Group 1 voltage-gated potassium channels}

Amongst the major classes of $\mathrm{K}^{+}$channels, the greatest diversity is to be found amongst VGKCs which activate in response to a change in membrane potential (Hille 2001). A large gene family encodes twelve subfamilies $\left(\mathrm{K}_{\mathrm{V}} 1-12\right)$ of the principle pore-forming $\alpha$ subunit (Figure 1.1). Structurally four $\alpha$-subunits, from the same subfamily, assemble into a tetrameric complex together with modifying auxiliary subunits, usually called $\beta$ subunits, to form a functional channel (Christie et al. 1990; Pongs 1992). Channel diversity is further enhanced by the assembly of $\alpha$-subunits into heteromultimeric channels with intermediate properties (Christie et al. 1990; Sheng et al. 1993; Scott et al. 1994). A wide range of biophysically distinct VGKCs is thus available to cells, which they may selectively express to meet their functional requirements. VGKCs are involved in numerous processes including setting resting membrane potentials, regulation of cellular excitability and synaptic transmission. The most extensively studied members are the $\mathrm{K}_{\mathrm{V}} 1$ or Shaker-related subfamily. Numerous studies have investigated the distribution of these channels with a view to establishing their role in normal function and disease states. 
Table 1.1: Ion channels identified in the GI tract

\begin{tabular}{|c|c|c|}
\hline Class & Subclass & Ion channel \\
\hline \multirow[t]{3}{*}{ G-protein coupled } & Class A (Rhodopsin - like) & $\begin{array}{l}\mathrm{M}_{2} / \mathrm{M}_{3} \mathrm{mAChR} \\
\text { Adrenergic } \\
\text { Histamine 1-4 } \\
5 \mathrm{HT}_{1,2,4,6,7} \\
\text { Bradykinin 1-4 } \\
\text { NK1-3 } \\
\text { Protease-activated (PAR) 1-4 } \\
\text { Prostaglandin 1-4 } \\
\text { Purinoceptors 1-11 }\end{array}$ \\
\hline & Class B (Secretin - like) & $\begin{array}{l}\text { Calcitonin } \\
\text { CRF 1-2 }\end{array}$ \\
\hline & Class C (mGluR-like) & $\begin{array}{l}\text { Glutamate 1-8 } \\
\text { GABA B1-2 } \\
\text { Ca-sensing } \\
\text { Taste T1-2R }\end{array}$ \\
\hline Ligand-gated & & $\begin{array}{l}\text { nAChR } \\
\text { P2X purinergic } \text { 5HT }_{3} \\
\text { NMDA } \\
\text { AMPA } \\
\text { Kainate } \\
\text { Glycine } \\
\text { GABAA }\end{array}$ \\
\hline Regulatory and sensory & & $\begin{array}{l}\text { TRP } \\
\mathrm{K}_{\mathrm{V}} \\
\mathrm{K}_{\mathrm{Ca}} \\
\mathrm{KcsA} \\
\mathrm{Na}_{\mathrm{V}} \\
\mathrm{L}, \mathrm{N}, \mathrm{P} / \mathrm{Q} \text {-type } \mathrm{VGCC} \\
\mathrm{Cl}^{-} \\
\text {ASICs }\end{array}$ \\
\hline Growth factor & & $\begin{array}{l}\text { Trk-A } \\
\text { RET }\end{array}$ \\
\hline
\end{tabular}

ASIC, acid sensing ion channel; CRF, corticotrophin-releasing factor; $\mathrm{K}_{\mathrm{Ca}}$, calcium-activated potassium channel; $\mathrm{K}_{\mathrm{V}}$, voltage-gated potassium channel; $\mathrm{mAChR}$, muscarinic acetylcholine receptor; $\mathrm{nAChR}$, nicotinic acetylcholine receptor; NK, neurokinin; TRP, transient receptor potential; Trk, tyrosine kinase receptor; VGCC, voltage-gated calcium channel 


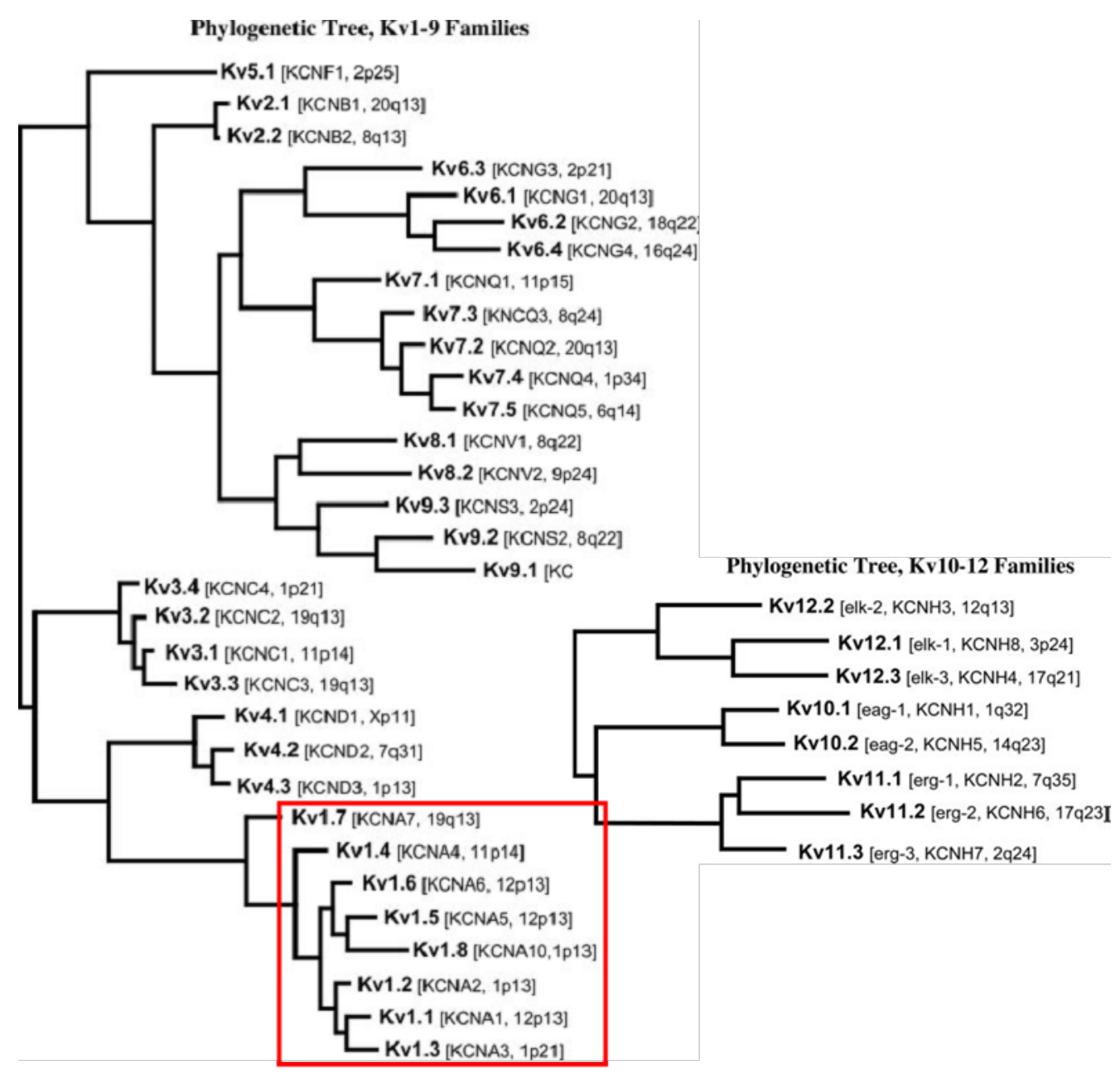

Figure 1.1: Phylogenetic tree for the voltage-gated potassium channel family. $T^{2} K_{V} 1$ subfamily, studied in this thesis, are outlined. (Gutman et al. 2005).

\subsubsection{2 $K_{V} 1$ channels of the GI tract}

In the CNS and peripheral neuromusculature regional and cellular $K_{V} 1$ channel distribution is well characterised (Beckh et al. 1990; Veh et al. 1995; Coleman et al. 1999; Chung et al. 2001; Glazebrook et al. 2002). In comparison, little is known of $\mathrm{K}_{V} 1$ subunit expression in neurons innervating the gut. Existing studies are described below. 


\section{Animal studies}

VGKC currents contribute to regulating excitability and repolarisation of action potentials in extrinsic sensory neurons innervating the oesophagus, stomach, ileum and colon. As a result, blocking these currents leads to a significant increase in action potential duration. Macroscopically, these currents can be divided into a rapidly inactivating current $\left(\mathrm{I}_{\mathrm{A}}\right)$ and a delayed rectifier or $\mathrm{I}_{\mathrm{K}}$ current. Pharmacological and molecular evidence points to a contribution of $\mathrm{K}_{\mathrm{V}} 1$ channels to both of these currents (Yoshimura et al. 1996; Glazebrook et al. 2002; Dang et al. 2004). Functionally within the gut, $\mathrm{K}_{\mathrm{V}} 1$ currents are associated with a number of processes including epithelial cell maturation and migration (Rao et al. 2002), nutrient absorption (McDaniel et al. 2001), modulation of enteric motor nerve excitability (Suarez-Kurtz et al. 1999; Vianna-Jorge et al. 2000) and generation of smooth muscle 'slow-wave' contractions (Thornbury et al. 1992; Thornbury et al. 1992; Carl 1995) As such, in a similar manner channel characterisation in the CNS and PNS, several studies have aimed to elucidate the specific $\mathrm{K}_{\mathrm{V}}$ subunit expression in the gut. In the mouse, Hatton and co-workers reported $\mathrm{K}_{\mathrm{V}} 1.1$ immunoreactivity expression in murine (as well as guinea pig and canine) myenteric ganglia and ICC (Hatton et al. 2001). However, others describe $\mathrm{K}_{\mathrm{V}} 1.1$ expression limited to enteroglial cells, with no immunoreactivity detected in neurons or ICC (Costagliola et al. 2009). Costagliola et al. also report $\mathrm{K}_{\mathrm{V}} 1.2$ expression in enteroglial cells and additionally in enteric neurons. $\mathrm{K}_{\mathrm{V}}$ channel currents contribute to the electrical slow wave generation of canine colonic smooth muscle cells (Thornbury et al. 1992; Thornbury et al. 1992; Carl 1995). Molecular studies of canine GI smooth muscle have subsequently identified $\mathrm{K}_{\mathrm{V}} 1.2, \mathrm{~K}_{\mathrm{V}} 1.4, \mathrm{~K}_{\mathrm{V}} 1.5$ and $\mathrm{K}_{\mathrm{V}} 1.6$ expression (Hart et al. 1993; Epperson et al. 1999). However, murine colonic smooth muscle cells express transcripts encoding $K_{V} 1.6$, but lack $\mathrm{K}_{\mathrm{V}} 1.4$ suggesting inter-species variability (Koh et al. 1999). 


\section{Human studies}

To date, a single study has identified $\mathrm{K}_{\mathrm{V}} 1$ channel subunits in the human neuromuscular apparatus. Recordings from isolated human oesophageal smooth muscle cells have revealed voltage-gated and $\mathrm{Ca}^{2+}$-activated potassium $\left(\mathrm{K}_{\mathrm{Ca}}\right)$ currents. Molecular analysis of the same tissue identified $\mathrm{K}_{\mathrm{V}} 1.2$ and 1.5 mRNA expression in both longitudinal and circular muscle layers (the expression of other $\mathrm{K}_{\mathrm{V}} 1$ channels was not investigated). Functionally, pharmacological blockade of the muscular voltage-gated potassium current caused an increase in resting tension, suggesting a role in setting resting membrane potentials. However, the agent used in this study (high concentration 4-aminopyridine) non-specifically blocks a number of potassium channel types and so the effect cannot be solely attributed to $\mathrm{K}_{\mathrm{V}} 1$ channels (Wade et al. 1999).

\section{3. 'CLASSICAL' ANTIBODY-MEDIATED NEUROLOGICAL DISEASES}

A number of peripheral neuromuscular diseases are known to be mediated by specific autoantibodies and are collectively known as autoimmune neuromuscular diseases (AINMDs). Although they do not primarily involve the ENS, they represent models by which the role of autoimmunity in GINMDs can be critically discussed. Best elucidated are diseases in which antibodies target peripheral ligand- or voltage-gated ion channels or associated proteins involved in neuromuscular transmission leading to several diseases e.g. myasthenia gravis, Lambert Eaton myasthenic syndrome and acquired neuromyotonia (Isaac's disease) (Vincent et al. 2006). Often termed autoimmune channelopathies, these contrast with genetic channelopathies in which a loss or decrease in function is conferred by gene mutation with similar phenotypic consequences (Terwindt et al. 1998). 


\subsubsection{MYASTHENIA GRAVIS}

Myasthenia gravis (MG) is the best understood autoimmune disorder of the nervous system and provides a 'bench-mark' against which putative autoimmune disorders may be judged. It is characterised by clinical features of fatigable muscle weakness which may initially affect the ocular muscles and variously spread to facial, bulbar and respiratory muscles and may also involve the limbs (Adams et al. 1993). Antibodies targeting postsynaptic nicotinic acetylcholine receptors (nAChRs) present on the surface membrane of muscle are detectable in greater than $85 \%$ of MG patients (Almon et al. 1974; Lindstrom et al. 1976; Vincent et al. 1985).

Around $10 \%$ of MG patients have a thymoma, and in these cases nAChR antibodies are always present (Vincent et al. 2006). Muscle weakness results from the functional loss of post-synaptic nAChRs secondary to receptor cross-linking by divalent antibodies and subsequent receptor internalisation (Drachman et al. 1978; Stanley et al. 1978) and complement-mediated degradation of the postsynaptic membrane (Engel 1979; Engel et al. 1981; Richman et al. 1993). Of those in whom anti-nAChR antibodies are not detectable (seronegative MG), around $40 \%$ have antibodies to a muscle-specific receptor tyrosine kinase (MuSK) (Wolfe et al. 2008) located at the neuromuscular junction (Valenzuela et al. 1995). MuSK is known to be important in clustering of AChRs during development, and although its role in adult muscle is less clear it appears to be necessary for maintenance of the post-synaptic membrane (Kong et al. 2004).

\subsubsection{LAMBERT EATON MYASTHENIC SYNDROME}

The Lambert Eaton myasthenic syndrome (LEMS) is a condition of proximal muscle weakness with accompanying autonomic dysfunction including dry mouth, erectile dysfunction, constipation and urinary symptoms. Approximately $50-60 \%$ of patients have 
an associated small cell lung carcinoma (SCLC). The underlying pathophysiology of LEMS is impaired calcium-dependent release of acetylcholine from motor nerve terminals (Elmqvist et al. 1968). Circulating antibodies against voltage-gated calcium channels (VGCCs) are found in around 90\% of LEMS patients (Lennon et al. 1995; Motomura et al. 1997) and result in the down-regulation of functional VGCCs (Pinto et al. 1998). VGCCs are composed of a number of pharmacologically and electrophysiologically distinct subtypes. Although antibodies have been described that bind to the L-, N- and P-/Q-VGCC subtypes (Meriney et al. 1996), only those directed against the P-/Q-subtype, which is responsible for pre-synaptic neurotransmitter release at the neuromuscular junction, have been shown to be pathogenic (Pinto et al. 1998). SCLCs have been shown to express functionally active P/Q-type VGCCs at the surface and it is thought that in these patients the neurological syndrome may result from a crossreactive immune response generated by tumour antigens (Viglione et al. 1995). The aetiology in the remaining $50 \%$ of patients without a tumour is unknown.

\subsubsection{ACQUIRED NEUROMYOTONIA}

Acquired neuromyotonia (NMT) is a disorder of spontaneous and continuous muscle fibre activity characterised by muscle stiffness, fasciculations, weakness and increased sweating (Isaacs 1961). It occurs due to motor nerve hyperexcitability and is associated with autoantibodies against VGKCs (Isaacs 1961; Newsom-Davis et al. 1993). Using current assays, antibodies to the $\mathrm{K}_{\mathrm{V}} 1$ family of VGKCs are detectable in $40 \%$ of patients with NMT (Hart et al. 2002).

Acquired NMT is associated with thymoma or SCLC in approximately $20 \%$ of cases (Newsom-Davis et al. 1993). Anti-VGKCs in NMT do not appear to block channel function directly as short incubation times have no effect. However, prolonged incubation of several hours reduces $\mathrm{K}^{+}$channel current amplitudes without altering gating kinetics 
(Sonoda et al. 1996; Nagado et al. 1999). This effect is also independent of added complement, or accelerated channel turnover, and degradation may occur secondary to cross-linking of channels by divalent antibodies (Tomimitsu et al. 2004).

\subsection{EVIDENCE FOR ANTIBODY MEDIATION OF AUTOIMMUNE DISEASES}

A number of clinical and experimental findings may help indicate that a disease has an autoimmune aetiology and these are central to subsequent discussion of the evidence for antibody mediation of GINMDs. Clinical features that are suggestive include onset of disease in adulthood, a history of autoimmunity in the patient or their family, an association with specific alleles of the human leukocyte antigen (HLA) gene complex, association with neoplasia, and clinical improvement following plasma exchange and / or immunosuppressive therapy (Vincent et al. 2006) (further discussed below). Such findings are also supported by finding evidence of immune cells in the target tissue although this is not an absolute requirement (Maselli et al. 1991; Vincent et al. 1999). In experimental terms, proof of disease causation should meet three broad criteria (Table $1.2)$. 
Table 1.2: Proof of disease causation

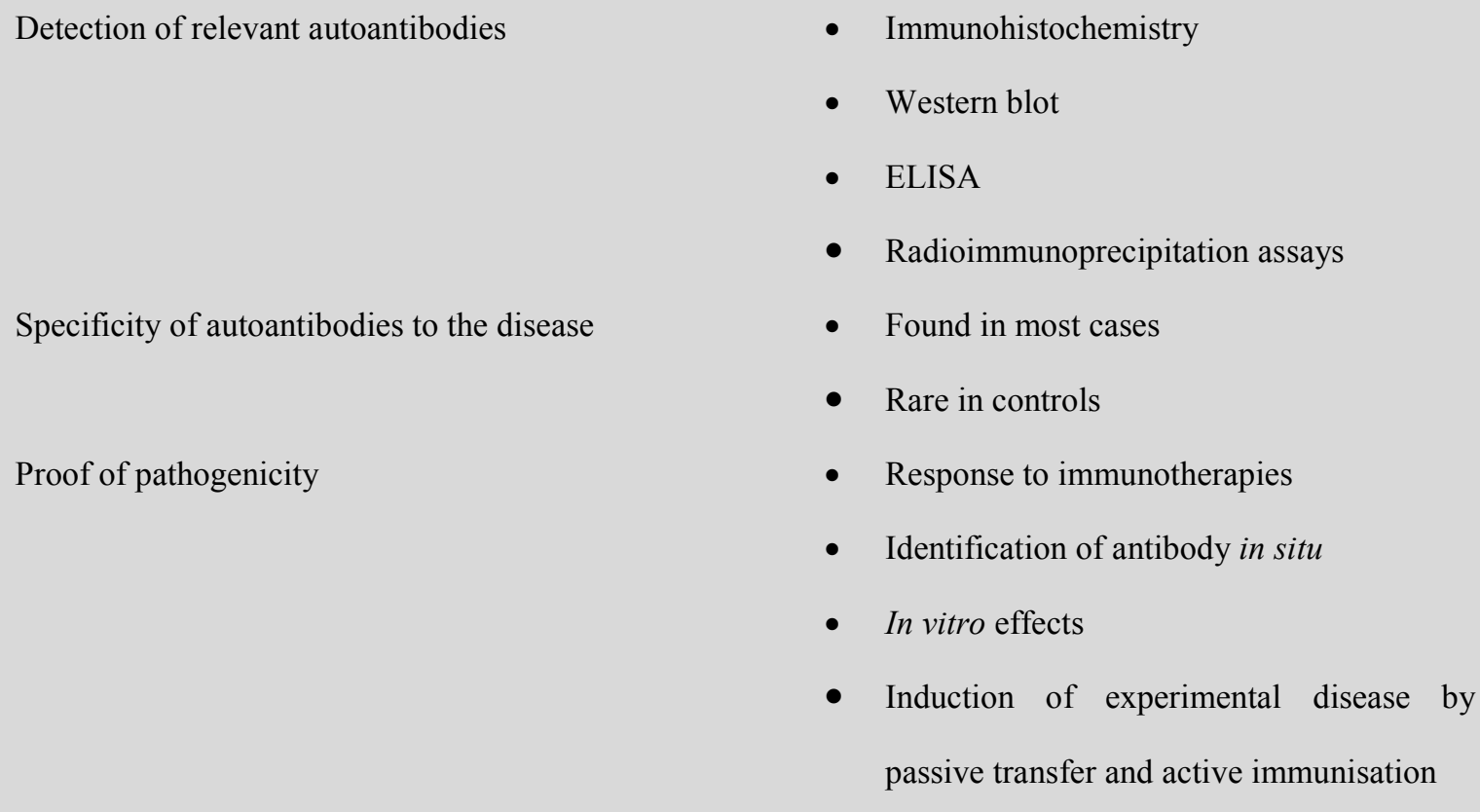

ELISA, enzyme-linked immunoadsorbent assay

\subsubsection{DETECTION OF AUTOANTIBODIES}

Autoantibodies may be detected by a number of techniques, the choice of which is often limited by the availability of specific antigens. Immunohistochemistry, western blotting, enzyme-linked immunoadsorbent assay (ELISA), radioimmunoprecipitation as well as functional assays are variously employed to measure antibodies in autoimmune neurological disorders (Tozzoli 2007). Each varies in accuracy, sensitivity and specificity and, if possible, a combination of methods should be used to confirm positive results (Vincent 2002). Although not discussed in detail here, it should especially be noted that in comparison with other methods, the sole use of ELISA or immunohistochemistry should be viewed cautiously because of the established risks of non-specific binding (Vincent 2002). Radioimmunoprecipitation assays, although acceptably specific, are 
dependent on the availability of suitable radiolabelled antigens of high specificity. The development of human cell lines (e.g. embryonic kidney fibroblasts) transfected to express channels or receptors of interest represents a means by which the binding of antibodies to the external surface of functionally active channels / receptors may be investigated (Blaes et al. 2000). This may be done by incubating cells expressing the target of interest with patient serum or by extracting the cells for fluorescent-labelled immunoprecipitation, and is perhaps now the assay of choice in detecting anti-channel antibodies, not only because functionality is strongly implied, but also because such assays may be more sensitive (Leite et al. 2008).

\subsubsection{SPECIFICITY FOR DISEASE}

Pathogenic autoantibodies would be expected to bind to the extracellular domain of a cell-surface antigen relevant to the disease in a significant number of affected individuals, and that such binding is rare in healthy and other disease controls. For instance it has already been noted that specific autoantibodies can now be demonstrated in almost all patients with MG (Chan et al. 2007), but are extremely rare in healthy controls and in patients with other autoimmune or neurological diseases.

\subsubsection{PROOF OF PATHOGENICITY}

The demonstration of circulating autoantibodies, even those specific for relevant cell surface targets does not necessarily prove a direct role in disease causation since these could have arisen secondary to tissue damage with exposure of host epitopes to the immune system (de Scheerder et al. 1989). To establish a direct pathogenic role a number of criteria must be fulfilled. These criteria originally established the role of nAChR antibodies in MG and have subsequently been applied to other putative autoimmune 
neurological disorders (for comprehensive review see (Vincent 2002). Each is discussed in the following sections:

\subsubsection{Response to immunotherapies}

Clinical improvement to plasmapheresis (plasma separation / exchange), intravenous immunoglobulin treatment or immunosuppressive therapies such as steroids and azathioprine strongly suggests a role for humoral autoimmunity. A further requisite should be that clinical response corresponds with a reduction in the titre of circulating antibodies as is seen in MG (Newsom-Davis et al. 1978) LEMS (Newsom-Davis et al. 1984) and acquired NMT (Shillito et al. 1995).

\subsubsection{Identification of antibody in situ}

Identification of autoantibodies bound at the neuromuscular junction has been demonstrated in $\mathrm{MG}$ in association with complement components and the membraneattack complex (Engel et al. 1977). The number of nAChRs was found to be inversely proportional to bound IgG confirming a role in nAChR loss (Engel et al. 1987). Following passive transfer to experimental animals, LEMS IgG has been identified at the active zones of the presynaptic motor end-plate membrane (Fukuoka et al. 1987).

\subsubsection{Modulation of antigen expression and / or functional effects in vitro}

Incubation with pathogenic IgG leads to altered tissue expression of the antigen and / or function of the antigenic target in cell lines, cell cultures and ex-vivo tissue. In LEMS, autoantibodies reduce the number of functional P/Q-type VGCCs on cell lines and cultures (Pinto et al. 1998). Similarly, muscle cells grown in the presence of MG IgG have a reduced number of functional nAChRs (Drachman et al. 1978). Serum or IgG 
from MG patients also inhibits the function of $\mathrm{nAChRs}$ on muscle cell lines by preventing the binding of ACh or other agonists (Riemersma et al. 1996). Incubation with NMT IgG results in repetitive action potentials in dorsal root ganglion cell cultures and reduced potassium currents in neuronal cell lines (Shillito et al. 1995; Nagado et al. 1999) mimicking disease electrophysiology.

\subsubsection{Passive transfer to experimental animals}

Injection of sera, plasma or immunoglobulins derived from affected patients into experimental animals results in passive transfer of the disease phenotype. Mice injected with MG IgG show muscle weakness and loss of muscle nAChRs (Toyka et al. 1977). The physiological effects on the peripheral and autonomic nervous system of LEMS can also be transferred to mice by injection of LEMS IgG (Prior et al. 1985; Lang et al. 1987; Waterman et al. 1997). Passive transfer of IgG from NMT patients results in increased quantal release of $\mathrm{ACh}$ at the neuromuscular junction and prolongation of the action potential (Shillito et al. 1995).

\subsubsection{Induction of experimental disease by immunisation}

An animal model of the disease is induced following immunisation with purified antigen. This confirmation of antibody pathogenicity was demonstrated at an early stage of the investigation of MG pathogenesis (Patrick et al. 1973). This proof has been difficult to achieve in other AINMDs due to an inability to produce relatively large quantities of pure antigen for immunisation. However, a preliminary animal model of LEMS produced by injecting mice and rats with synaptosomes containing (although not exclusively) L-, Nand P-/Q-VGCCs isolated from the electric organ of the Japanese electric ray, Narke japonica, has gone some way to addressing this problem (Kim et al. 1998). 
These criteria will now be applied to primary and secondary GINMDs. From a perspective of hierarchy of evidence, secondary GINMDs are discussed first.

\subsection{EVIDENCE FOR HUMORAL AUTOIMMUNITY IN SECONDARY GINMDS}

\subsubsection{PARANEOPLASIA}

Clinical and histological evidence suggests an autoimmune basis for GINMD-related motor dysfunction associated with neoplasms: (1) histological examination of GI tissue from patients with paraneoplastic motility disorders reveals myenteric ganglionitis / plexitis, suggestive of some form of immune-mediated process. These include lymphocytic and plasmacellular infiltration, enteroglial cell proliferation and neuronal degeneration and loss, progressing to aganglionosis in the most severe cases (Schuffler et al. 1983; Chinn et al. 1988; Schobinger-Clement et al. 1999; Lee et al. 2001); (2) patients with SCLC and GI dysmotility often have co-existing neurological disorders including peripheral neuropathies, cerebellar degeneration and limbic encephalitis, which are thought to have an autoimmune origin (Chinn et al. 1988; Lucchinetti et al. 1998; Vernino et al. 1998); (3) several potentially pathogenic 'paraneoplastic autoantibodies' reactive against antigens expressed by tumour cells and neurons have been identified, which in combination with neurological disorders, including GI symptoms, strongly suggest the presence of an underlying malignancy (Lucchinetti et al. 1998; Pande et al. 1999; Schobinger-Clement et al. 1999; Lee et al. 2001).

\subsubsection{Detection of autoantibodies}

Paraneoplastic GI dysmotility, most commonly secondary to a SCLC, is associated with seropositivity for anti-neuronal autoantibodies. Most common in this context are type 1 
anti-neuronal nuclear autoantibodies (ANNA-1, also known as anti-Hu), which recognise neuron-specific RNA binding proteins expressed by central and peripheral neurons, including enteric neurons, and SCLC tumour cells (Bell et al. 1976; Lucchinetti et al. 1998; Lee et al. 2001). Antibodies targeting cell-surface channels have been detected in smaller numbers of cases, including anti-N-type VGCCs with SCLC, retroperitoneal Bcell lymphoma, ovarian and breast carcinoma (Lee et al. 2001; Dhamija et al. 2008), antiP/Q-type VGCCs with SCLC (Pardi et al. 2002), anti-VGKCs with thymoma (Viallard et al. 2005) and anti-ganglionic $\alpha 3 \mathrm{nAChR}$ antibodies with SCLC, thymoma, bladder and rectal carcinoma (Vernino et al. 2000).

\subsubsection{Specificity for disease}

Autoantibodies are commonly found in GI dysfunction secondary to an underlying malignancy. A study of patients at the Mayo clinic with confirmed paraneoplastic GI dysfunction found 10 of 11 patients were seropositive for one or more "paraneoplastic autoantibodies, including 8 positive for anti-Hu and one positive for anti-N-type VGCC (Lee et al. 2001). In a further study of various types of GI dysmotility, anti-Hu were were always indicative of a malignancy (Dhamija et al. 2008). It is important to note that GI symptoms frequently precede diagnosis of a tumour, which may remain occult despite intensive evaluation (Lucchinetti et al. 1998).

\subsubsection{Proof of pathogenicity}

\section{Response to immunotherapies}

Paraneoplastic neurological, including gastrointestinal, symptoms may respond to immunomodulatory therapy. Plasmaphaeresis leading to a striking improvement in bowel motility and a correlating reduction in circulating antibody levels has been reported in a case of intestinal pseudo-obstruction associated with neuromyotonia and high titre 
ANNA-1 and a case of slow transit constipation associated with thymoma and antiVGKC antibodies (van Vuurden et al. 2005; Viallard et al. 2005). Intravenous immunoglobulin treatment has also achieved significant improvement in paraneoplastic GI dysmotility associated with anti- $\alpha 3$ nAChR antibodies and ANNA-1 (Dhamija et al. 2008).

Modulation of antigen expression and / or functional effects in vitro

On the basis of their intracellular antigenic target (as discussed above), ANNA-1 are unlikely to be directly pathogenic although nuclear epitopes could be exposed by prior inflammation / cell damage. A study based on a small series of patients with paraneoplastic GI dysmotility has suggested that ANNA-1 positive sera are able to trigger neuronal apoptosis in vitro suggesting a possible role in enteric neuronal degeneration (De Giorgio et al. 2003) (Figure 1.2). Anti-channel antibodies are more likely to exert a pathogenic effect due to the accessibility of the antigenic target. It is known from the study of peripheral neuromuscular disease that autoantibodies against VGKC, P/Q VGCC and $\alpha 3 \mathrm{nAChRs}$ are pathogenic. However, functional effects of these autoantibodies in the context of paraneoplasia have not been investigated. 

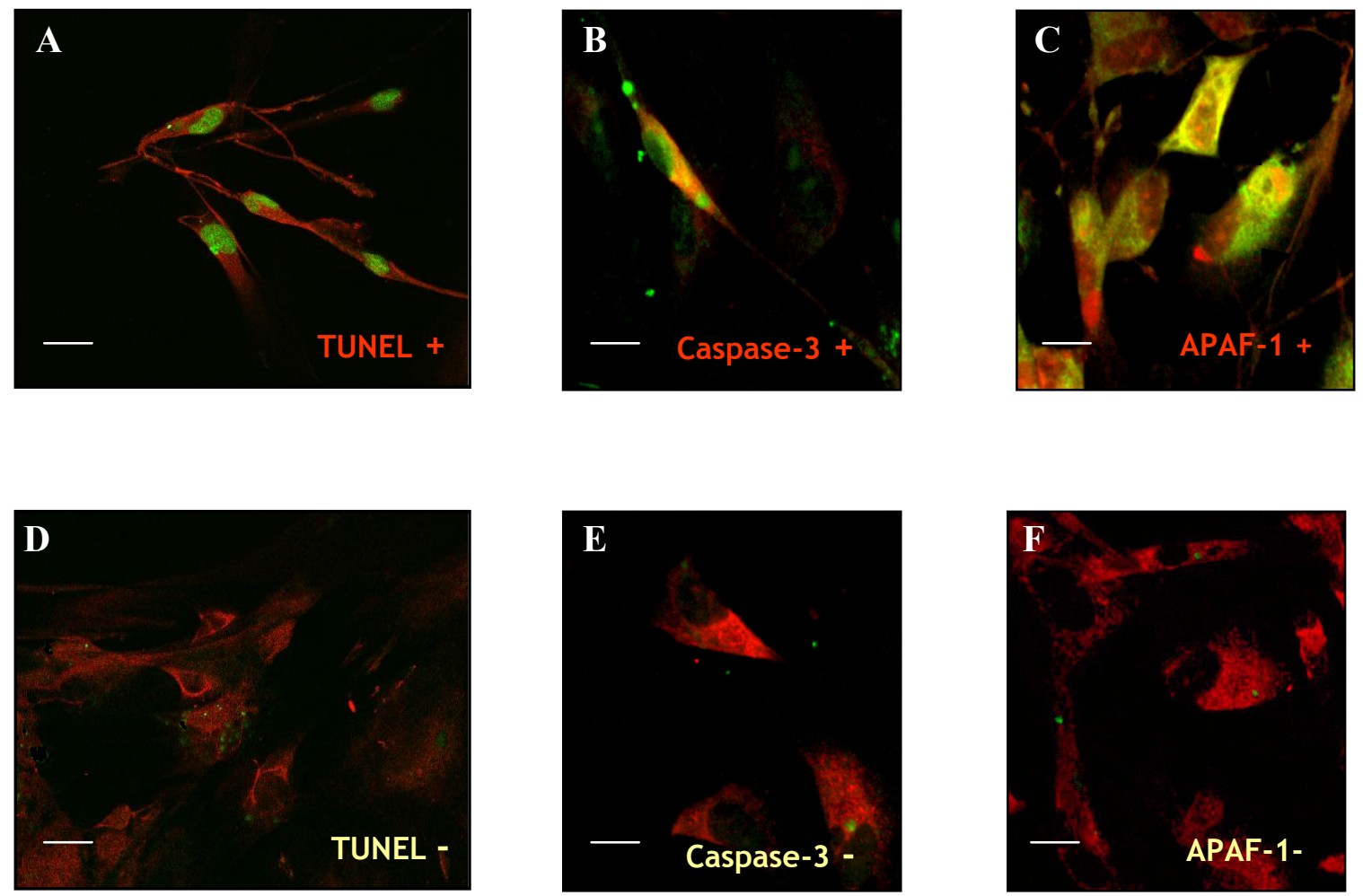

Figure 1.2: Pro-apoptotic effects of anti-Hu antibody on cultured enteric neurons.

Representative immunofluorescent, confocal double-labelling photomicrographs of NSE-labelled cultures myenteric neurons (red) and three markers of cellular apoptosis: TUNEL, caspase-3 and APAF-1 (A-F). Exposure of myenteric neurons to commercial anti-Hu antibody (48 hrs) evoked: A: an intense TUNELpositivity (green) in nuclei of NSE-labelled myenteric neurons (red), B: caspase-3 immunoreactivity (green) and C: APAF-1 (green). In contrast, TUNEL, caspase-3 and APAF-1 were absent in NSE-positive myenteric neurons (red) exposed to fetal calf serum or control (blood donor) serum (D-E, respectively). APAF-1 $=$ apoptotic protease activating factor $1, \mathrm{NSE}=$ neuron-specific enolase, TUNEL $=$ Terminal deoxynucleotidyl transferase dUTP nick end labelling. Scale bar $=20 \mu \mathrm{m}$. Courtesy of Prof. R De Giorgio; (De Giorgio et al. 2003). 


\subsubsection{CONNECTIVE TISSUE DISORDERS}

Severe bowel dysfunction occurs in a proportion of patients with connective tissue disorders and is most commonly associated with systemic sclerosis (scleroderma, SSc). Any region of the gut may be affected, although oesophageal dysmotility is most commonly recognised (Rose et al. 1998). Although in later stages of the disease smooth muscle atrophy and fibrosis are clearly evident, manometric studies suggest that a neuropathic mechanism may be the initial event (Battle et al. 1981; Rees et al. 1982).

\subsubsection{Detection of autoantibodies}

Scleroderma is associated with anti-nuclear autoantibodies and more recently has been linked with other, non-nuclear antibodies such as anti-endothelial cell, anti-fibrillin-1, anti-metalloproteinases and anti-platelet-derived growth factor receptor (Tan et al. 2000; Sato et al. 2003; Ahmed et al. 2006; Baroni et al. 2006). The sera of scleroderma patients has also been found to contain antibodies capable, in immunofluorescent assays, of binding myenteric neurons (Howe et al. 1994). Anti-muscarinic AChR (mAChR) autoantibodies have also been identified in patients with SSc and GI dysfunction (see below for further discussion) (Goldblatt et al. 2002; Kawaguchi et al. 2009).

\subsubsection{Specificity for disease}

Circulating anti-myenteric neuronal autoantibodies demonstrated by Howe et al., using indirect immunofluorescence, were found in 16 of 41 scleroderma patients and 11 of these had clinical or laboratory manifestations of GI dysfunction. However, 19 of 25 patients without anti-myenteric antibodies also had GI dysfunction, casting doubt on a direct causative role (Howe et al. 1994). IgG against mAChRs were detected by 
radioimmunoprecipitation in 7 of 9 scleroderma patients with gastrointestinal symptoms. The two negative patients had only mild or no GI symptoms (Goldblatt et al. 2002).

\subsubsection{Proof of pathogenicity}

\section{Response to immunotherapies}

Plasmaphaeresis combined with immunosuppression resulted in amelioration of severe gastrointestinal symptoms 4 of 15 patients with gastrointestinal symptoms and corresponded with a decline in anti-nuclear antibody titres (Dau et al. 1981; Akesson et al. 1988).

\section{Modulation of antigen expression and / or functional effects in vitro}

IgG purified from scleroderma sera has been shown to inhibit muscarinic cholinergic smooth muscle contraction in isolated colon providing a potential mechanism for GI dysmotility. In this in vitro study the putative target was considered to be the $\mathrm{M}_{3}$ $\mathrm{mAChR}$, as $\mathrm{IgG}$ and a specific $\mathrm{M}_{3}$-receptor antagonist inhibited, in a similar manner, evoked colonic muscle contraction. However, from this observation alone the IgG effect cannot be attributed solely to $\mathrm{M}_{3} \mathrm{mAChR}$ antagonism as the agent used to evoke the contractions, carbachol (a muscarinic receptor agonist) also activates the $\mathrm{M}_{2}$ receptor subclass. Futhermore, the authors note in their discussion that they have failed to show direct binding to $\mathrm{M}_{3} \mathrm{mAChRs}$ transfected and expressed on the surface of mammalian cells or to virally produced recombinant receptors (Goldblatt et al. 2002) (see section 1.5.4 for further description). Nevertheless, the presence of antibodies against the $\mathrm{M}_{3}$ mAChR was investigated using an ELISA to screen for binding to a synthetic peptide corresponding to the second extraellular loop domain of the human receptor. In a study of 14 scleroderma patients with GI involvement, sera from all were found to bind (Kawaguchi et al. 2009). The target autoantigen in scleroderma patients remains to be satisfactorily resolved and this area of study highlights the possible discrepencies 
between peptide-based immunoassays and more complex functional or cell-based binding assays.

Passive transfer to experimental animals

Passive transfer of purified IgG from a patient with a specific variant of SSc (CREST syndrome) and anti-neuronal antibodies has successfully produced intestinal myoelectrical disruption in a rat model providing good evidence of pathogenic circulating antibodies. However, the generalisability of this to other varients of SSc is unknown (Eaker et al. 1999).

\subsubsection{TYPE 1 DIABETES}

Measurable disturbances in GI motility, in particular delayed gastric emptying, are a common feature of diabetes mellitus (Keshavarzian et al. 1987). Such changes may result from diabetic neuropathy affecting intinsic (enteric) and / or extrinsic (autonomic) neurons or from changes in intestinal smooth muscle (for review see (Chandrasekharan et al. 2007).

\subsubsection{Detection of autoantibodies}

Autoimmune mechanisms involved in diabetic neuromuscular dysfunction have rarely been investigated. Although autoantibodies to the neuronal and islet cell antigen glutamic acid decarboxylase (GAD) are detectable in around 70\% of patients with recent onset type 1 diabetes (Seissler et al. 1993), GAD is an intracellular enzyme and would therefore not present a target in intact cells. The presence of functional antibodies in a group of patients with type 1 diabetes and gastrointestinal symptoms has been investigated pharmacologically and identified IgG against the L-type VGCC (Jackson et al. 2004). 


\subsubsection{Specificity for disease}

Using the techniques described below, 8 of 16 patients had functional anti-VGCC autoantibodies. Of these, 7 had reported symptomatic gastrointestinal dysfunction. Such findings were not found in appropriate positive and negative controls (Jackson et al. 2004).

\subsubsection{Proof of pathogenicity}

Modulation of antigen expression and / or functional effects in vitro

Diabetic IgG produced a disruption of migrating motor complexes in intact colonic segments which was similar to the effect produced by an L-type VGCC agonist. Furthermore, the IgG was shown to competitively reduce binding of the specific L-type channel antagonist dihydropyridine (Jackson et al. 2004).

\section{Passive transfer to experimental animals}

Severe disruption of the migrating motor complex was demonstrated in colons isolated from mice injected with diabetic IgG containing the putative L-type calcium channel agonistic antibodies (Jackson et al. 2004).

Thus, experimental evidence for anti-channel autoimmunity in diabetic patients with GI dysfunction exists, albeit from a single study. Whilst the microvascular and neurological consequences of poor glycaemic control are of course significant and account for diabetic complications, the finding of functional anti-channel antibodies in these patients warrants further investigation. 


\subsubsection{CHAGAS' DISEASE}

Infection by the protozoan parasite Trypanosoma cruzi causing South American Trypanosomiasis (Chagas' disease) (Chagas 1909) is a significant public health issue in the developing world, affecting 16-18 million of the Latin American population (Kirchhoff 2003). The most damaging and established sequel is that of fatal cardiomyopathy, however the digestive tract is also commonly affected with extensive destruction of ganglion cells (Ribeiro et al. 1998; da Silveira et al. 2007) leading to altered motility manifest as functional obstruction with eventual progression to hypertrophy of the smooth muscle and marked dilatation (i.e. megaoesophagus and megacolon) (Koberle 1968). Phenotypically, chronic disease manifestations vary geographically, reflecting the heterogeneity of T.cruzi strains and their pathogenic sequalae. Megaoesophagus and megacolon are prominent complications in southern countries of South America, whereas these complications are rarer in Central and northern regions (Miles et al. 2009).

The role of antibody-mediated autoimmune pathology in Chagas' disease is speculative given that there is more evidence for direct damage caused by the organism itself and by cell-mediated mechanisms (Kierszenbaum 2005). Nevertheless, some autoantibodies to both cell surface and intracellular targets have been detected (Kierszenbaum 1999).

\subsubsection{Detection of autoantibodies}

There is good evidence that circulating autoantibodies present in some patients with Chagas' disease are capable of modifying cellular events downstream of muscarinic cholinergic receptor agonism. Autoantibodies modifying muscarinic cholinergic neuromuscular activity were first identified in Chagas' disease patients with secondary dysautonomic syndrome where patient IgG caused an in vitro reduction in cardiac 
muscular tone which could be blocked by atropine (Goin et al. 1994). Immunoblotting and ELISA subsequently identified the putative antigenic target as the second extracellular loop (the main immunogenic region) of the $\mathrm{M}_{2} \mathrm{mAChR}$ (Goin et al. 1997).

\subsubsection{Specificity for disease}

The dysautonomia described in Chagasic cardiomyopathy and GI dysmotility is strongly associated with the presence of autoantibodies as detected by the anti- $\mathrm{M}_{2}$ peptide ELISA with $90 \%$ of patients with a measurable autonomic disturbance testing positive, compared with less than 30\% with no disturbance and in no healthy controls (Goin et al. 1997; Ganzinelli et al. 2009). These autoantibodies have been detected in $84 \%$ of patients with chagasic achalasia versus $21 \%$ of those with Chagas' disease but no oesophageal sequalae and 28\% in idiopathic achalasia (Goin et al. 1999). Similarly, in those with chagasic colonic dysfunction, autoantibodies were detected in $87 \%$ compared to $24 \%$ of those without megacolon (Sterin-Borda et al. 2001). However, using the same ELISA, these antibodies have been found in a high proportion of patients with other types of heart disease including $62 \%$ of patients with idiopathic cardiomyopathy, $46 \%$ with hypertensive heart disease and 48\% with rheumatic valvular disease (Fu et al. 1993; Zhang et al. 2002). The presence of the same autoantibodies in non-parasitic cardiomyopathies increases the likelihood of a secondary phenomenon arising as a result of tissue damage.

\subsubsection{Proof of pathogenicity}

\section{Response to immunotherapies}

The chronic phase of the disease may be associated with a low parasitic load suggesting a further immuno-inflammatory response could contribute to disease pathology. In a rat model of chronic disease a short treatment with infliximab (a monoclonal antibody which 
neutralises the activity of tumour necrosis factor- $\alpha$ ) reduced myocarditis severity, indicating an immuno-pathological role for this cytokine (Perez et al. 2009). However, the primary role of parasite induced tissue damage in both the acute and chronic phase of infection, which is limited by host cellular and humoral immune responses, suggests that the use of immunomodulatory therapy would be counterproductive.

Modulation of antigen expression and / or functional effects in vitro

Chagasic IgG has repeatedly been shown to have in vitro effects which are mediated via the $\mathrm{M}_{2} \mathrm{mAChR}$ signalling pathway. In oesophageal and colonic muscle preparations, IgG produced concentration-dependent increases in basal tone which were sensitive to $M_{2}$ mAChR antagonism (Goin et al. 1999; Sterin-Borda et al. 2001). It also acted as a partial agonist, mimicking the effect of the muscarinic agonists carbachol and pilocarpine in a non-competitive manner (Goin et al. 1994; Goin et al. 1997). Radioligand binding assays investigating the interaction of chagasic $\operatorname{IgG}$ with the muscarinic receptor indicated chagasic IgG acts as an allosteric antagonist of the muscarinic receptor. It decreased the number of receptors available for binding $\left(\mathrm{B}_{\max }\right)$ without an associated effect on the affinity constant $\left(\mathrm{K}_{\mathrm{D}}\right)$ of the radioligand (Goin et al. 1994; Sterin-Borda et al. 2001). Further support of receptor agonism was provided by studies examining downstream effects of muscarinic activation in the presence of IgG. Incubation of cardiac or GI smooth muscle with IgG revealed pertussis toxin-sensitive decreased tissue levels of cyclic adenosine monophosphate (cAMP) and an increase in cyclic guanosine monophosphate (cGMP). This is consistent with muscarinic receptor activation (Goin et al. 1997; Goin et al. 1999; Sterin-Borda et al. 2001).

L-type voltage-gated calcium channel (VGCC) currents in isolated ventricular myocytes are also reduced in the presence of chagasic antibodies, in the absence of other agonists (Hernandez et al. 2003). An increase in inducible nitric oxide synthase (iNOS) and cyclooxygenase-2 (COX-2) gene transcription is observed in response to chagasic $\mathrm{IgG}$ application, increasing prostaglandin E2 $\left(\mathrm{PGE}_{2}\right)$ and nitric oxide (NO) production 
(Ganzinelli et al. 2009). This has pathological relevance to the end-organ inflammation seen in chronic Chagas disease.

\section{Passive transfer to experimental animals}

To date, the transfer of disease phenotype to experimental animals by injection of immunoglobulins from affected patients has not been demonstrated.

\section{Induction of experimental disease by immunisation}

Active immunisation of mice with $\mathrm{M}_{2} \mathrm{mAChR}$-derived synthetic peptides led to the production of functional anti-peptide antibodies. In these animals basal heart rate was unaffected, however carbachol induced bradycardia was significantly increased in comparison to controls, and atropine induced tachycardia was blunted suggesting an agonistic function (Peter et al. 2005).

\subsection{EVIDENCE FOR HUMORAL AUTOIMMUNITY IN PRIMARY GINMDS}

\subsubsection{IDIOPATHIC ACHALASIA}

Idiopathic achalasia of the oesophagus is an idiopathic motility disorder characterised by aperistalsis of the oesophageal body and incomplete lower oesophageal sphincter (LOS) relaxation on swallowing leading to dysphagia, regurgitation, chest pain and weight loss (Willis 1674; Hertz 1915; Castell 1976). Myenteric denervation of the distal oesophagus extending into the stomach is characteristically seen at histological examination (Csendes et al. 1992) and is selective for inhibitory nitric oxide (Mearin et al. 1993; De Giorgio et al. 1999; Zarate et al. 2006) and vasoactive inhibitory polypeptide (Aggestrup et al. 1983; Sigala et al. 1995) releasing motor neurons resulting in unopposed excitatory activity. Familial, infectious, genetic and autoimmune mechanisms have all been postulated to contribute to the development of achalasia (Park et al. 2005). 


\subsubsection{Detection of autoantibodies}

An autoimmune pathogenesis is suggested by lymphocytic inflammatory infiltrates in the myenteric plexus (Goldblum et al. 1994; Clark et al. 2000) and disease association with specific HLA alleles (Ruiz-de-Leon et al. 2002; Latiano et al. 2006). Immunohistochemical techniques have repeatedly demonstrated anti-neuronal antibodies binding both submucosal and myenteric ganglia in the serum of achalasia patients (Storch et al. 1995; Verne et al. 1997; Moses et al. 2003). Autoantibodies against specific neuronal, glial and muscle antigens studied using radioimmunoprecipitation assays have identified nAChR, VGKC and N-type VGCC antibodies in a subset of primary achalasia patients (Kraichely et al. 2009).

\subsubsection{Specificity to disease}

Indirect immunofluorescence screening puts the prevalence of anti-neuronal antibodies at 12-64\% compared with 0-9\% of healthy controls (Storch et al. 1995; Verne et al. 1997; Ruiz-de-Leon et al. 2002; Moses et al. 2003; Bruley des Varannes et al. 2006). This proportion has been reported to rise significantly in patients with specific HLA genotypes. One hundred percent of female and $67 \%$ of male carriers of the DQA $1 * 0103$ DQB1*0603 heterodimer with achalasia were found to have antineuronal antibodies (Ruiz-de-Leon et al. 2002). Significant associations between DQB1*0602, DRB1*15 (Verne et al. 1999), DQB1*0502 and DQB1*0601 alleles (Latiano et al. 2006) and achalasia have also been reported, confirming an immunologic predisposition to the disease. However, although anti-neuronal antibodies appear specific to enteric neurons they bind both inhibitory and excitatory myenteric neurons and also submucosal ganglia with equal affinity, thus not accounting for the selective loss of inhibitory motor neurons. Regional specificity, which would also support a pathogenic role is lacking as antibodies label myenteric gangia from all regions of the GI tract (Storch et al. 1995; Verne et al. 
1997; Moses et al. 2003). Whilst reasonably disease-specific i.e. not present in control groups such as megacolon, Hirschsprung's disease, oesophageal cancer and MG (Storch et al. 1995), anti-neuronal antibodies have also been found in as many as $50 \%$ of patients with gastro-oesophageal reflux disease (GORD) casting doubt on their primary role in disease causation (Moses et al. 2003). Furthermore, no correlation has been found between patient age or clinical findings and the presence of anti-neuronal antibodies (Storch et al. 1995; Verne et al. 1997). A study of the prevalence of specific antineuronal / muscle antibodies using radioimmunoprecipitation assays (VGKC, GAD, Ntype VGCC and muscle nAChR) found a significant proportion (26\%) had one or more. Most strikingly, $21 \%$ of primary achalasia patients versus $2.5 \%$ of healthy controls had detectable anti-GAD antibodies. Only one of these had documented diabetes. The clinical significance of this finding remains to be ascertained (Kraichely et al. 2009).

\subsubsection{Proof of pathogenicity}

\section{Modulation of antigen expression and / or functional effects in vitro}

A novel approach using pre-incubation of gastric fundal tissue with sera from patients with achalasia demonstrated a subsequently altered myenteric neurochemical phenotype relevant to current understanding of disease pathogenesis (Bruley des Varannes et al. 2006). Specifically, inhibitory nitric oxide synthesising (NOS) neurons were reduced in number, whilst the proportion of excitatory choline acetyltransferase (ChAT) synthesising neurons increased, findings which in keeping with both incomplete LOS relaxation and histological findings. Achalasia sera used for both immunohistochemistry and in vitro motility studies revealed a correlation between the decrease in the proportion of NOS immunoreactive neurons and electrical field stimulation induced nitric oxide NOdependent relaxation in circular muscle strips. The decrease in NOS immunoreactive neurons may be due to an effect of the serum on neuronal NOS (nNOS) expression at mRNA and/or protein level as the changes occurred rapidly (after 16-18 hours of 
incubation). Although GORD serum did not alter neuronal chemical phenotype, suggesting a specific effect of achalasia serum, this methodology is not yet well validated and no signs of neuronal degeneration were found as seen histologically in active disease. In addition it is unknown which serum factors are responsible for this effect (Boeckxstaens 2006).

\subsubsection{SEVERE INTESTINAL DYSMOTILITY}

Severe intestinal dysmotility (SID) describes a group of disorders where the bowel fails to propel its contents in the absence of an obstructed lumen. At the most severe end of this spectrum are patients with episodic or chronic signs mimicking a mechanical obstruction: abdominal pain, distension, nausea/vomiting, and intractable constipation. These are termed chronic idiopathic intestinal pseudo-obstruction (CIPO) (Dudley et al. 1958). This diagnosis is based on a combination of the above symptoms with radiological evidence of gut dilatation and air-fluid levels during acute episodes (Gibbons et al. 1978; Hirsh et al. 1981; Gilchrist et al. 1985) and/or abnormal small bowel motility (Stanghellini et al. 1987; Di Lorenzo 1999). Enteric dysmotility describes patients with demonstrably abnormal small bowel contractility, but no history of frankly obstructive episodes. These patients may be at an early stage in the progression of CIPO, or perhaps be those in a quiescent period of a relapsing / remitting pseudo-obstriction (Wingate et al. 2002; Lindberg et al. 2009). Whilst the pathophysiology of these disorders remains poorly understood, CIPO may be classified according to recognised histopathological abnormalities of enteric smooth muscle (myopathies), ENS (neuropathies) and of ICC (mesenchymopathies). Several degenerative and inflammatory myopathic and neuropathic phenotypes have been described in children and adults on the basis of histopathology (Smith et al. 1992; Ruuska et al. 2002; Knowles et al. 2004; Tornblom et al. 2005; Knowles et al. 2009). Cases of both myopathic and neuropathic CIPO secondary to presumed autoimmune processes have been reported. Histological features 
in most cases suggest the disease may be caused by local T-cell-mediated responses directed against enteric neurons or smooth muscle, but there is also evidence for autoantibody-mediated pathogenesis.

\subsubsection{Detection of autoantibodies}

Serologically, CIPO has been associated with anti-nuclear, anti-neutrophil cytoplasmic antibody (ANCA), anti-DNA, anti-smooth muscle and anti-neuronal antibodies (ANAs) (Ruuska et al. 2002; Ghirardo et al. 2005). Anti-enteric neuronal antibodies have been described in two patients with intestinal obstruction due to acquired aganglionosis. Whilst these antibodies also bound CNS neurons in a pattern characteristic of ANNA-1, and were also indistinguishable on Western blots, immunostaining of enteric neurons displayed a quite different pattern, being mostly cytoplasmic with only a weak nuclear component. Neither patient had CNS involvement and the autoimmune injury appeared limited to the ENS (Smith et al. 1997).

\subsubsection{Specificity for disease}

Using indirect immunofluorescence techniques, the largest study to date found 6 out of 25 (24\%) patients with CIPO had anti-enteric neuronal antibodies (binding to both neuronal nuclei and cytoplasm) (de Giorgio et al. 2008). Other studies have identified specific autoantibodies in small numbers of patients, including one patient with antiVGKC and one with anti-ganglionic $\alpha 3 \mathrm{nAChR}$ antibodies. Both of these patients had histologically-proven inflammatory enteric neuropathy, although in these cases diarrhoea was the chief complaint (Tornblom et al. 2007). Using immunoprecipitation assays to identify antibodies against $\alpha 3 \mathrm{nAChRs}$ in patients with various types of dysautonomia, Vernino et al. identified antibodies in 3 of 34 patients with a diagnosis of idiopathic GI dysmotility, although at a lower titre than other seropositive patients with more severe, 
diffuse dysautonomias, suggesting these patients may have a limited form of autoimmune autonomic neuropathy (AAN) (further discussion below). (Vernino et al. 2000). Low, but positive titres of antibodies to P/Q VGCCs were also found in 4 out of 10 patients with CIPO (Knowles et al. 2002).

\subsubsection{Proof pathogenicity}

\section{Response to immunotherapies}

A number of case reports highlight remission of pseudo-obstructive episodes following immunomodulatory therapy. In these patients abdominal symptoms responded to immunosuppression with steroids and / or cyclosporin, which were started after failure of prokinetic therapy and surgical intervention and often after histological assessment revealed an inflammatory neuropathy or myopathy (Smith et al. 1997; De Giorgio et al. 2002; Ruuska et al. 2002; Dewit et al. 2008). Whilst a cell-mediated immune response is clearly present in these cases, the humoral immune system may also be activated, as evidenced by a paediatric case of intestinal pseudo-obstruction associated with antismooth muscle and anti-neutrophil cytoplasmic antibodies. In this instance a good clinical response to immunosuppressive therapy was also associated with a reduction in circulating autoantibody levels (Ruuska et al. 2002). Whilst not immunomodulatory, a patient with chronic gastropaeresis (delayed-gastric emptying) who was seropositive for anti- $\alpha 3 \mathrm{nAChR}$ antibodies reported significant improvement in GI symptoms after commencing acetylcholinesterase inhibitor therapy, indicating a pathogenic role for these antibodies. (Pasha et al. 2006).

\section{Modulation of antigen expression and / or functional effects in vitro}

A functional effect of CIPO sera containing ANAs has been suggested by de Giorgio et al (de Giorgio et al. 2008). They found evidence that sera containing ANAs stimulates neuronal autophagy (a cellular response to adverse conditions where by injured 
organelles such as mitochondria are sequestered in autophagosomes) associated with neuronal stress. This effect was reduced by removal of IgG suggesting a direct role for immunoglobulins. However, this pathway is normally cytoprotective in limiting the production of reactive oxygen species and the pathophysiologic significance in CIPO is unclear.

Passive transfer to experimental animals and induction of experimental disease by immunisation

Using active immunisation and subsequent passive transfer techniques, the pathogenicity of anti-a3 nAChR antibodies has been investigated. Rabbits immunised with a recombinant fusion protein corresponding to the nAChR $\alpha 3$ subunit produced autoantibodies reactive with ganglionic nAChRs with development of a generalised AAN with prominent gastrointestinal dysmotility including gastroparesis and intestinal pseudoobstruction (Lennon et al. 2003; Vernino et al. 2003). Severity of findings correlated with antibody levels, and immunohistochemistry revealed reduced numbers of myenteric plexus neurons and reduced immunoreactivity for nAChRs at neuronal surfaces and synapses (Vernino et al. 2003). Passive transfer of rabbit IgG containing anti- $\alpha 3 \mathrm{nAChR}$ antibodies to mice replicated active immunisation findings, confirming the role of antibodies in mediating AAN (Vernino et al. 2004). It should be noted, however, that passive transfer of sera of patients with severe autonomic neuropathy and anti- $\alpha 3$ nAChRs, even from those with highest titres of antibodies, failed to replicate these findings. The reason for this discrepancy is unclear (Vernino et al. 2004).

\subsubsection{SLOW TRANSIT CONSTIPATION}

A proportion of patients with severe constipation will have a reduction in the propulsive capacity of all or part of the colon that on the basis of transit studies is defined as slow transit constipation (STC). STC includes a clinically heterogeneous group of patients, the 
majority of whom present in childhood and who are nearly always female (Preston et al. 1986). Others present in later life with onset of symptoms de novo or often attributed to pelvic surgery or childbirth (Knowles et al. 1999; Knowles et al. 2000). A plethora of aetiologies have been proposed to underlie STC including primary neuromuscular / ICC dysfunction, absorptive, hormonal and psychological abnormalities, with inconclusive results (Knowles et al. 1999; Knowles et al. 2000). In those presenting with de novo adult-onset STC an autoimmune aetiology remains an under-explored possibility.

\subsubsection{Detection of autoantibodies}

Validated immunoprecipitation assays detected high titres of anti-VGKC antibodies in two of 11 sera tested suggesting anti-neuronal antibodies may be important in certain acquired STC patients.

\subsubsection{IDIOPATHIC MEGACOLON}

Idiopathic megacolon describes a group of patients with intractable constipation associated with rectal or colonic dilatation in the absence of mechanical obstruction or evident cause (Bodian et al. 1949). Several pathological abnormalities of nerve, ICC and smooth muscle have been reported including immune-mediated myenteric ganglionitis associated with neuronal degeneration (De Giorgio et al. 2002).

\subsubsection{Detection of autoantibodies}

In contrast to acquired megacolon associated with systemic diseases such as Chagas' disease (see above), the possibility of a humoral immune response against enteric neuromuscular elements contributing to the pathogenesis of idiopathic megacolon has not been investigated. 


\subsection{SUMMARY AND KNOWLEDGE GAPS}

The recognition of autoantibody-mediated channelopathies has opened new avenues in the investigation and management of peripheral neuromuscular disorders, whilst in cancer-associated neurological syndromes the detection of antibodies to neoplastic antigens has increased early diagnosis and treatment of the underlying malignancy. It is clear that whilst there is some evidence for autoantibody-mediated GI dysfunction in primary and secondary GINMDs, this is not strong in comparison with AINMDs such as myasthenia gravis, particularly in reference to specificity of antibody detection and proof of pathogenicity. These data are summarised in Table 1.3. In general the evidence is stronger for secondary, in particular paraneoplastic, than primary GINMDs, largely due to a paucity of information regarding the association of anti-channel antibodies with primary GINMDs. In determining a role for humoral autoimmunity in GINMDs the original paradigms generated by the investigation of peripheral neuromuscular disorders such as myasthenia gravis should be applied.

Of direct relevance to anti-channel autoimmunity contributing to GI dysfunction is the lack of data concerning channel expression in the gut. Although investigation of enteric channel expression points towards diversity equal to that of the CNS and PNS, in comparison to these systems knowledge regarding the ENS is in its infancy. This knowledge gap is especially true of the human ENS as initial investigations in small mammals are only beginning to be translated. As well as providing information regarding the functional biology of the gut, these studies will provide an expanding list of candidates for autoimmune interaction. 
Table 1.3: Evidence for humoral autoimmunity in AINMD and GINMD

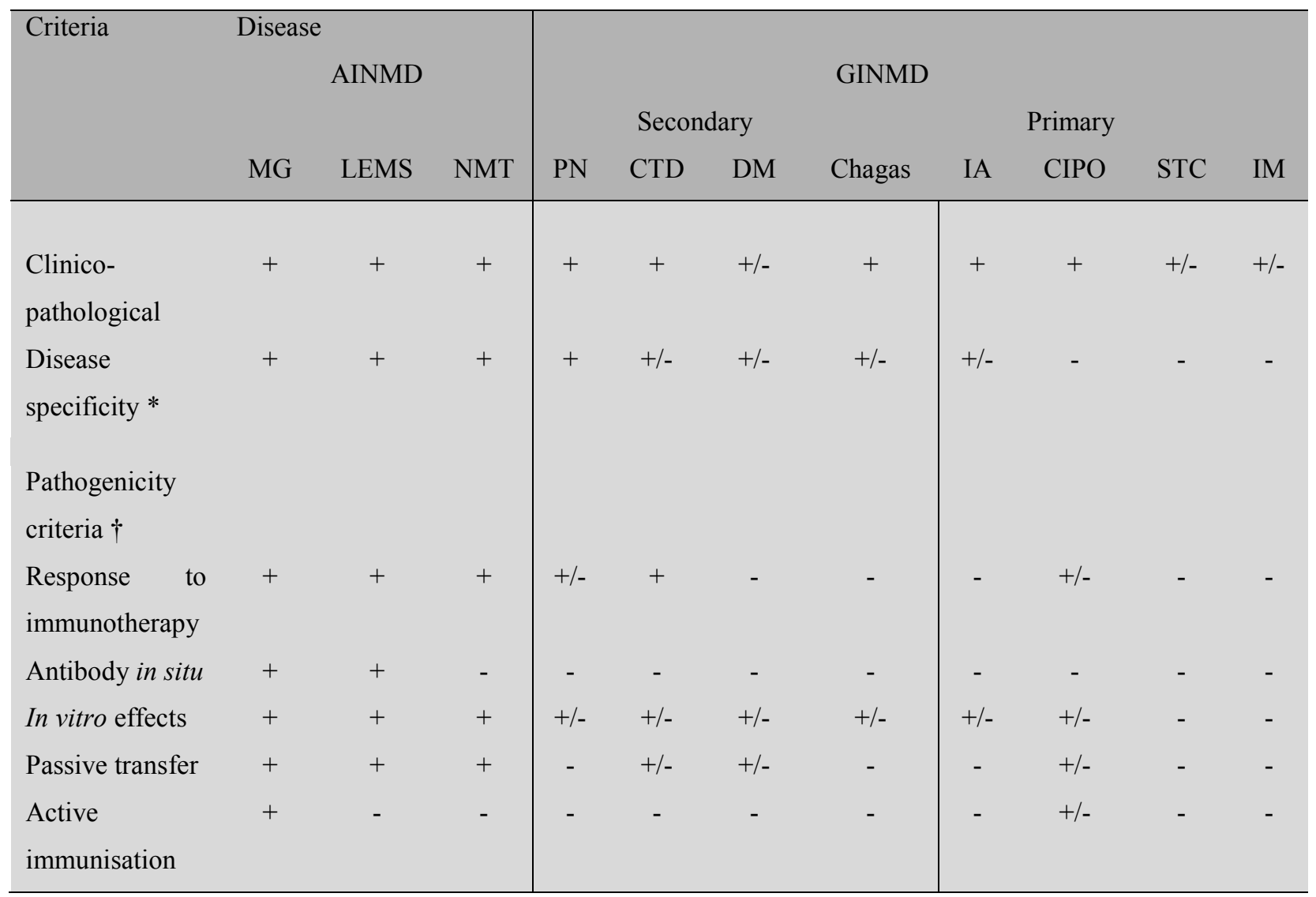

$*$ cut off of $50 \%$ disease cases with antibodies; $\uparrow$ defined on the basis of + good quality evidence from more than one study; +/- weaker evidence usually arising from only one study; - no evidence to date. MG, myasthenia gravis; LEMS, Lambert-Eaton myasthenic syndrome; NMT neuromyotonia; PN, paraneoplastic; DM, diabetes mellitus; CTD, connective tissue disorders; IA, idiopathic achalasia; CIPO, chronic idiopathic intestinal pseudo-obstruction; STC, slow transit constipation; IM, idiopathic megabowel. 


\subsection{AIMS}

The aims of this project were:

1. To investigate the presence of autoantibodies directed to enteric ion channels in patients with neuromuscular disorders of the gut leading to severe dysmotility.

2. To investigate whether autoantibodies (if present) are pathogenic.

3. To further elucidate the profile of expressed ion channels in the human enteric neuromusculature.

\subsection{HYPOTHESES}

1. Autoantibodies to one or more type of ion channel are present in a subset of patients with gastrointestinal neuromuscular disease.

2. As in the CNS and PNS, these autoantibodies are pathogenic and directly contribute to GI neuromuscular dysfunction.

3. Ion channels expressed by the CNS and PNS which are known targets of humoral autoimmune interaction are also present in the ENS. 


\section{MATERIALS AND METHODS}

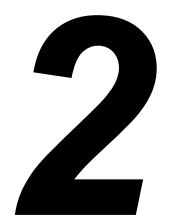

This chapter covers all specific methods used. Details of routine techniques, such as standard histochemical staining, can be found in the appendices.

\subsection{ETHICAL APPROVAL AND COLLABORATIONS}

The original application established collaborations with one international and one UK academic institution. These were the Departamento de Clinica Medica Faculdade de Medicina, University of Sao Paulo, and the Neurosciences Group, John Radcliffe Hospital, Oxford. The former facilitated collection of sera from Brazilian patients with Chagas' disease and from community controls from several clinics in Fortaleza and surrounding areas for anti-channel antibody analysis. Following the acquisition of Home Office approval, the necessary arrangements were made for the sera to be transported to the UK. The Neurosciences Group provided the facilities and technical support for screening all sera for circulating anti-channel antibodies. The collection and use of sera and tissue studies was approved by the Royal Free Hospital \& Medical School Research Ethics Committee in April 2006. Following amendments to the original application, a further site (Homerton University Hospital, London) was added for the collection of human gastrointestinal tissue. 
The work contained within this thesis is covered by the following ethics committee references:

06/Q0501/52

06/Q0501/52/AM01

06/Q0501/52/AM02

[See Appendix 1.0 for correspondence and confirmatory documents].

\subsection{DETECTION OF AUTOANTIBODIES IN PATIENTS WITH GINMDS}

\subsubsection{COLLECTION OF PATIENT SERA}

Venous whole blood $(20 \mathrm{ml})$ was taken by antecubital fossa venepuncture and centrifuged for $10 \mathrm{~min}$ at $3,500 \mathrm{rpm}$. Serum was removed and stored at $-80^{\circ} \mathrm{C}$ in Eppendorf aliquots (approximately $4 \mathrm{~mL} /$ patient) until use.

\subsubsection{Achalasia}

Twenty-one patients with achalasia (14 female, aged 26-72, median 55 years) who had undergone investigation at the Upper GI Unit at the Royal London Hospital were screened for autoantibodies. All patients had undergone manometric investigation of their oesophageal motility and were documented as having 'established' achalasia with manometric findings of abnormal peristalsis and incomplete lower oesophageal sphincter relaxation on swallowing (Figures 2.1 and 2.2) (Castell 1976; Pandolfino et al. 2005). 


\subsubsection{Severe intestinal dysmotility}

Twenty-seven patients with severe intestinal dysmotility ( 22 female, aged 18-62, median 45 years) who attended the pseudo-obstruction outpatient clinic at the Royal London Hospital were studied. The median number of years from presentation to donation of a blood sample for this study was 10 (range 3-40). Chronic idiopathic intestinal pseudoobstruction (CIPO) was diagnosed in 7 patients ( 5 female) on a basis of typical gastrointestinal symptoms (abdominal pain, severe constipation, abdominal distension, nausea and vomiting, weight loss; see Table 2.1), sub-occlusive events with radiological signs mimicking mechanical obstruction (air/fluid levels with or without dilatation on plain abdominal radiograph) and abnormal small bowel manometry (Dudley et al. 1958; Wingate et al. 2002) (Table 2.2). Twenty patients had abnormal intestinal motility but no history of sub-occlusive episodes and were classified as enteric dysmotility (Wingate et al. 2002; Lindberg et al. 2009). Abdominal pain and bloating were the most prominent features in both groups. As expected and previously reported (Lindberg et al. 2009), nutritional support was required in a greater proportion of patients with CIPO (6/7) than ED (8/20). The predominant manometric abnormality in CIPO patients was a neuropathic motor pattern (as evidenced by abnormal qualitative and quantitative measures of the migrating motor complex (Stanghellini et al. 1987). The ED group had an almost equal frequency of neuropathic and abnormal but indeterminate manometric patterns. Histological reports from full-thickness biopsies were available in all CIPO and 9 ED patients who had undergone laparoscopic biopsy (see Table 2.3). A greater number of biopsy samples were reported to be normal in the ED group than the CIPO group. An equal number (3/7) of CIPO patients had a neuropathy as had a myopathy. Alpha-actin deficiency (included by convention as a myopathy (Knowles et al. 2010) was the most common finding in the ED group, and two patients had both an alpha-actin deficiency and an inflammatory neuropathy. There was a poor correlation between histological and manometric findings. 


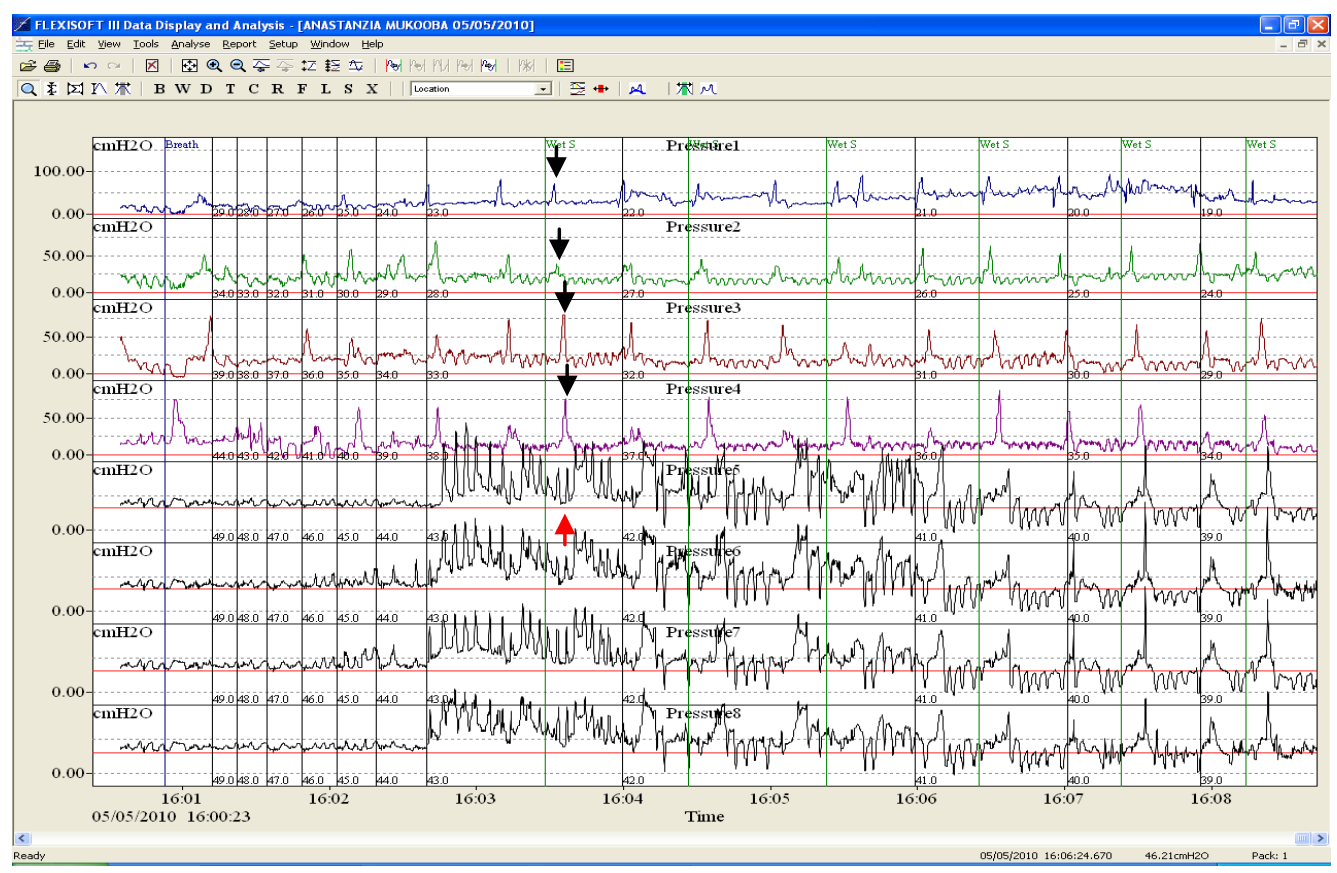

Figure 2.1: Oesophageal manometry tracing showing a normal swallow. Trace shows propagation of contraction following a wet swallow (black arrows) and relaxation of lower oesophageal sphincter (red arrow) 


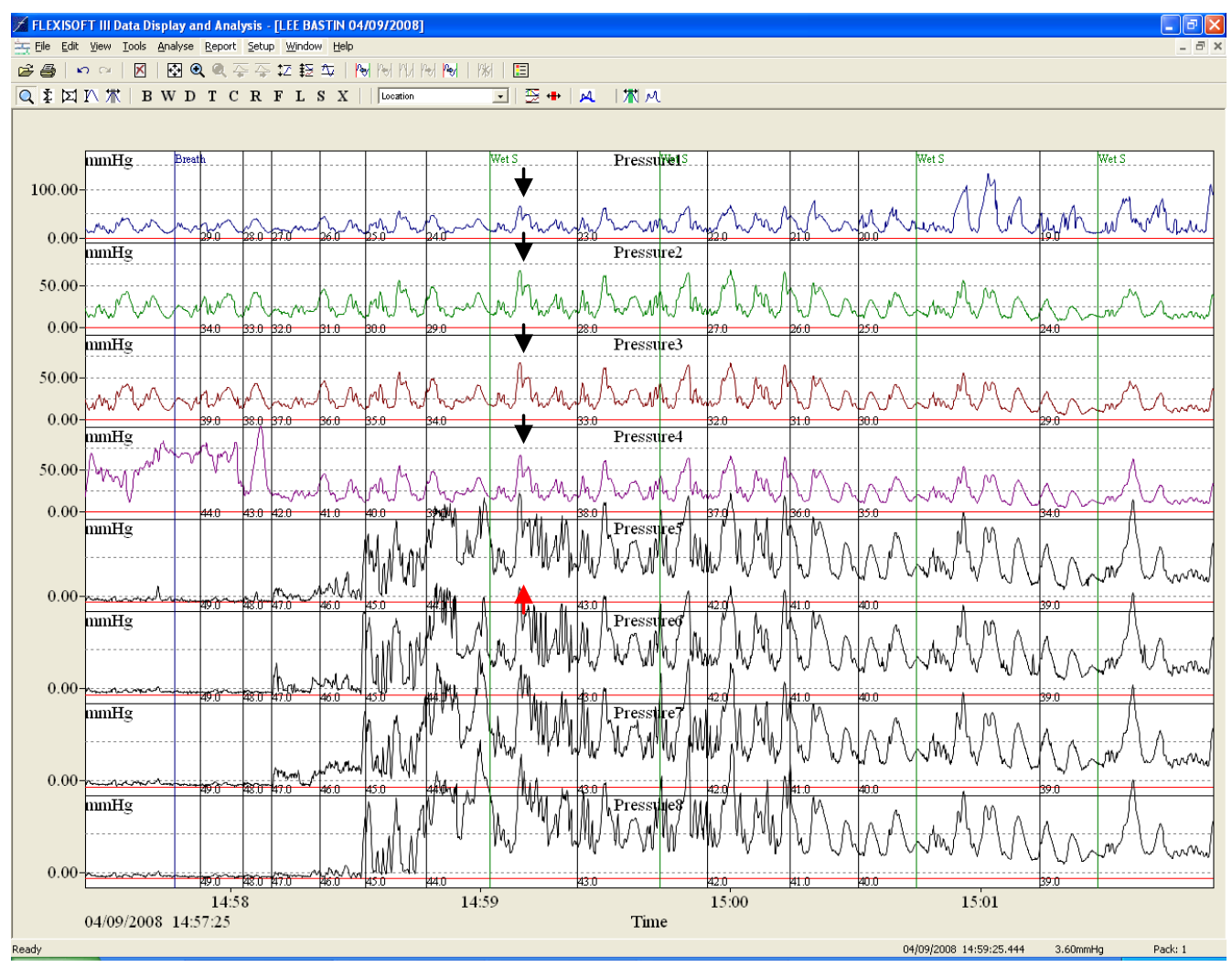

Figure 2.2: Oesophageal manometry tracing from a patient with achalasia. Trace shows simultaneous contractions following a wet swallow (black arrows) and failure of relaxation of lower oesophageal sphincter (red arrow) 
Table 2.1: Clinical features of severe intestinal dysmotility

\begin{tabular}{|c|c|c|c|c|}
\hline \multirow[t]{3}{*}{ Clinical features } & \multicolumn{4}{|c|}{$\mathbf{N}$} \\
\hline & \multicolumn{2}{|c|}{$\operatorname{CIPO}(n=7)$} & \multicolumn{2}{|c|}{$\operatorname{ED}(n=20)$} \\
\hline & Presentation & Current & Presentation & Current \\
\hline Weight loss & 6 & 3 & 8 & 3 \\
\hline Dysphagia & 0 & 0 & 1 & 2 \\
\hline Nausea & 6 & 3 & 2 & 4 \\
\hline Vomiting & 2 & 1 & 6 & 9 \\
\hline Reflux & 0 & 0 & 1 & 3 \\
\hline Bloating & 5 & 6 & 15 & 13 \\
\hline Abdominal pain & 6 & 7 & 10 & 17 \\
\hline Constipation & 5 & 2 & 7 & 6 \\
\hline Diarrhoea & 0 & 3 & 0 & 1 \\
\hline Irregular bowel habit & 0 & 0 & 0 & 4 \\
\hline Urgency & 0 & 0 & 0 & 1 \\
\hline Incontinence & 0 & 0 & 0 & 2 \\
\hline Incomplete evacuation & 0 & 0 & 0 & 1 \\
\hline \multicolumn{5}{|l|}{ Feeding requirements } \\
\hline None & \multicolumn{2}{|c|}{1} & \multicolumn{2}{|c|}{12} \\
\hline Enteral & \multicolumn{2}{|c|}{2} & \multicolumn{2}{|c|}{2} \\
\hline Parenteral & \multicolumn{2}{|c|}{4} & \multicolumn{2}{|c|}{4} \\
\hline Both & \multicolumn{2}{|c|}{0} & \multicolumn{2}{|c|}{2} \\
\hline
\end{tabular}




\section{Table 2.2: Investigation findings in patients with SID}

\begin{tabular}{|c|c|c|}
\hline \multirow{2}{*}{ Investigation findings } & \multicolumn{2}{|c|}{$\mathbf{N}$} \\
\hline & CIPO $(n=7)$ & $\operatorname{ED}(n=20)$ \\
\hline \multicolumn{3}{|c|}{ Radiography (plain radiograph $+/$ - contrast $+/$ - CT) } \\
\hline Normal & 0 & 6 \\
\hline Dilated small bowel & 7 & 0 \\
\hline Malrotated small bowel & 0 & 2 \\
\hline Dilated large bowel & $1^{*}$ & 0 \\
\hline Delayed transit & 0 & 2 \\
\hline Increased transit & 0 & 1 \\
\hline Not reported & 0 & 9 \\
\hline \multicolumn{3}{|l|}{ Small bowel manometry } \\
\hline Neuropathic & 5 & 8 \\
\hline Myopathic & 0 & 1 \\
\hline Abnormal indeterminate & 2 & 10 \\
\hline Not done & 0 & $1 \dagger$ \\
\hline
\end{tabular}

* One CIPO patient had radiological evidence of small and large bowel dilatation

$\dagger$ Small bowel manometry failed in one patient due to retrograde propulsion 
Table 2.3: Histopathological findings in full-thickness jejunal biopsies from patients with SID

\begin{tabular}{lcc}
\hline \multicolumn{1}{c}{ Histopathological abnormalities } & CIPO (n=7) & \\
& & ED (n=20) \\
\hline Normal & $\mathbf{1}$ & $\mathbf{4}$ \\
Visceral neuropathy & $\mathbf{3}$ & $\mathbf{2}$ \\
Degenerative neuropathy & 1 & 0 \\
Inflammatory neuropathy & 2 & $2^{*}$ \\
& & \\
Visceral myopathy & $\mathbf{3}$ & $\mathbf{5}$ \\
a-actin deficiency & 1 & $4^{*}$ \\
Degenerative leiomyopathy & 1 & 0 \\
Inflammatory leiomyositis & 0 & 0 \\
Vascular myopathy & 0 & 0 \\
Hollow visceral myopathy & 0 & 0 \\
Inclusion body myopathy & 1 & 1 \\
Interstitial cell abnormalities & & 0 \\
Total & 0 & $\mathbf{9}$ \\
\hline
\end{tabular}

* Two patients had both an $\alpha$-actin deficiency and an inflammatory neuropathy 


\subsubsection{Healthy controls}

Ten control samples were obtained from individuals (5 female, age 18 - 70, median 59) attending outpatient clinics at the Royal London Hospital who had no present or past history of GI, connective tissue or autoimmune disorder.

\subsubsection{Chagas' disease}

Sixty-one Brazilian patients with Chagas' disease were tested, forty-five male, aged 2886, median 50 years. All patients were recruited from clinics in and around the city of Fortaleza, in north-east Brazil (Figure 2.3). Although the strain of T.cruzi was not identified in these cases, in this region the sequel of chronic disease is most commonly cardiac damage (see Chapter 1 for discussion of phenotypic variability related to T.cruzi strain heterogeneity). However, within this cohort, each chronic manifestation is represented and eight sub-groups were defined using the following criteria: those without organ involvement; those with achalasia, colonic disease or both; those with cardiomyopathy or ECG changes, and those with heart and gut manifestations. These patients were further divided into those that had undergone previous treatment with antiT.cruzi drug therapy with Benzonidazol and those that had not (see Table 2.3). It would have been desirable to obtain more details regarding the past medical history of these patients. However, despite written requests this information was not forthcoming. 
Table 2.4: Disease-subgroups of Chagas' disease patients

\begin{tabular}{lccc}
\hline & Total & Untreated & Treated \\
Chagas' disease sub-groups & & & \\
\hline No sequalae & 29 & 16 & 13 \\
Achalasia & 9 & 7 & 2 \\
Colon disease & 4 & 1 & 3 \\
Achalasia + colon disease & 1 & 0 & 1 \\
Heart disease & 8 & 7 & 1 \\
Heart disease + achalasia & 5 & 3 & 2 \\
Heart disease + colon disease & 3 & 1 & 1 \\
Heart disease + achalasia + colon disease & 2 & 1 & \\
\cline { 2 - 4 } & $\mathbf{6 1}$ & & 2 \\
\hline
\end{tabular}

\subsubsection{Brazilian controls}

Twenty control sera samples were obtained from healthy subjects (11 female, aged 22 76 , median 39 years) from the same communities as those who provided Chagas' disease samples. 


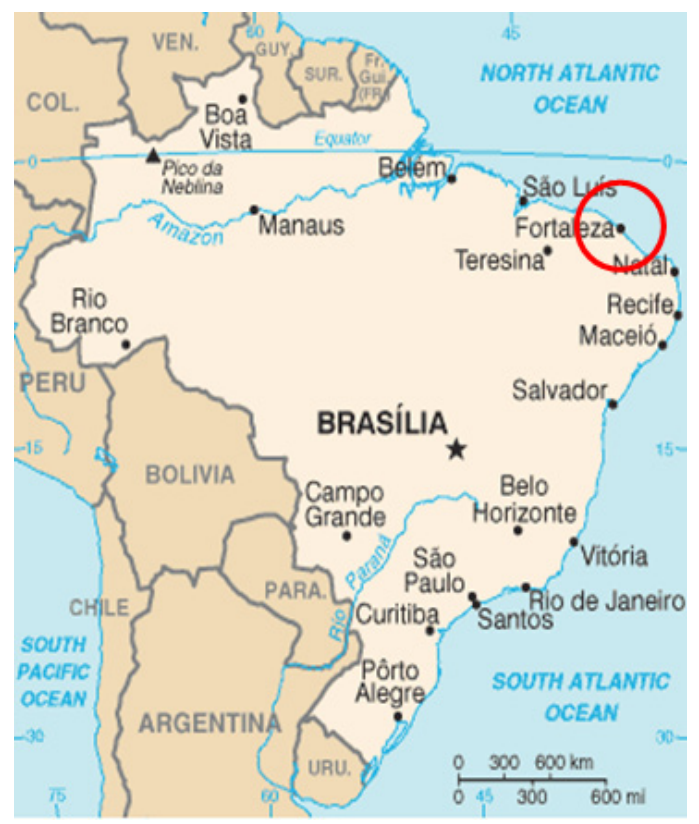

Figure 2.3: Map of Brazil. All chagasic blood samples were obtained from clinics serving Fortaleza and surrounding areas in the north-east of the country (circled).

\subsubsection{Disease controls}

Disease control sera were obtained from patients attending the John Radcliffe Hospital, Oxford. These were from patients with: autoimmune autonomic neuropathy $(n=2)$, Lambert-Eaton myasthenic syndrome (LEMS; $\mathrm{n}=2$ ) and neuromyotonia $(\mathrm{NMT} ; \mathrm{n}=2)$, containing anti-ganglionic $\alpha 3$ nicotinic acetylcholine receptor ( $\alpha 3 \mathrm{nAChR})$, anti-voltagegated calcium channel (VGCC) and anti-voltage-gated potassium channel (VGKC) antibodies respectively (see Table 2.4 for details of all subjects). 
Table 2.5: Basic clinical data and demographics for patients included in assay studies

\begin{tabular}{|c|c|c|c|c|}
\hline Study group & Description & $\mathbf{N}$ & $\operatorname{Sex} F / M$ & $\begin{array}{l}\text { Age median } \\
\text { (range) }\end{array}$ \\
\hline Achalasia & $\begin{array}{l}\text { Incomplete LOS relaxation on } \\
\text { swallowing } \\
\text { Abnormal peristalsis }\end{array}$ & 21 & $14 / 7$ & $55(26-72)$ \\
\hline $\begin{array}{l}\text { Severe intestinal } \\
\text { dysmotility }\end{array}$ & $\begin{array}{l}\text { CIPO }(n=7) \\
\text { Enteric dysmotility } \\
(\mathrm{n}=20)\end{array}$ & 27 & $22 / 5$ & $45(18-62)$ \\
\hline Chagas' disease & $\begin{array}{l}\text { No sequalae }(\mathrm{n}=29) \\
\text { Achalasia }(\mathrm{n}=17) \\
\text { Colon disease }(\mathrm{n}=9) \\
\text { Heart disease }(\mathrm{n}=18)\end{array}$ & 61 & $16 / 45$ & $50(28-86)$ \\
\hline Healthy subjects & $\begin{array}{l}\text { UK negative controls } \\
\text { Brazilian negative controls }\end{array}$ & $\begin{array}{l}10 \\
20\end{array}$ & $\begin{array}{c}5 / 5 \\
11 / 9\end{array}$ & $\begin{array}{l}59(18-70) \\
39(22-76)\end{array}$ \\
\hline $\begin{array}{l}\text { AAN } \\
\text { LEMS } \\
\text { NMT }\end{array}$ & $\begin{array}{l}\text { Positive controls: } \alpha 3-\mathrm{nAChR} \text { assay } \\
\text { positive controls: VGCC assay } \\
\text { positive controls: VGKC assay }\end{array}$ & $\begin{array}{l}2 \\
2 \\
2\end{array}$ & $\begin{array}{l}1 / 1 \\
1 / 1 \\
2 / 0\end{array}$ & $\begin{array}{l}50(31,68) \\
52(46,58) \\
42(34,50)\end{array}$ \\
\hline
\end{tabular}

$\mathrm{AAN}=$ autoimmune autonomic neuropathy, $\mathrm{ED}=$ enteric dysmotility, $\mathrm{CIPO}=$ chronic idiopathic intestinal pseudo-obstruction, LEMS $=$ Lambert-Eaton myasthenic syndrome, $\mathrm{nAChR}=$ nicotinic acetylcholine receptor, $\mathrm{NMT}=$ neuromyotonia, $\mathrm{VGCC}=$ voltage-gated calcium channel, VGKC $=$ voltage-gated potassium channel 


\subsubsection{ANTI-NEURONAL AUTOANTIBODY IMMUNOFLUORESCENT ASSAY}

\subsubsection{Background to methodology}

Seropositivity for anti-neuronal autoantibodies (ANAs) is a diagnostic marker of occult malignancy in the presentation of paraneoplastic CIPO, which usually precedes the diagnosis of a tumour (Lennon et al. 1991; Lucchinetti et al. 1998; Lee et al. 2001). To confirm the absence of such autoantibodies in our cohort of patients, all those with SID were screened for ANAs. Three types of ANAs associated with paraneoplastic neurological dysfunction are commonly screened for: Anti-Hu, anti-Yo and anti-Ri. Anti$\mathrm{Hu}$, also known as anti-neuronal nuclear antibody type 1 (ANNA-1), the most important with regards to paraneoplastic GI dysmotility, recognise a family of pan-neuronal RNA binding proteins, thus immunolabelling neuronal nuclei, and are chiefly associated with small-cell lung cancer (SCLC) (Bell et al. 1976; Graus et al. 1986; Altermatt et al. 1991). Anti-Yo, also known as anti-Purkinje cell cytoplasmic antibody (PCA) show cytoplasmic immunoreactivity of cerebellar Purkinje cells and are associated with gynaecological and breast cancer (Greenlee et al. 1983; Graus et al. 1988). Anti-Ri (ANNA-2), primarily associated with breast cancer, also bind neuron-specific RNA binding proteins but in contrast to anti-Hu, label neuronal nuclei of central neurons only (Budde-Steffen et al. 1988; Luque et al. 1991). ANAs are detected by standard indirect immunofluorescence where sera are incubated with sections of nervous tissue (typically mammalian cerebellum) and binding detected using an anti-human secondary antibody conjugated to a fluorophore (see section 2.4.1 for general immunohistochemical technical description). The pattern of immunoreactivity (nuclear vs. cytoplasmic) suggests the type of antibody present, which may then be confirmed by western blotting. 


\subsubsection{Indirect immunofluorescence}

Sera samples were diluted 1 in 50 in PBS, applied to monkey cerebellar sections (Inova Diagnostics Inc., CA, USA) and incubated for $20 \mathrm{~min}$ at room temperature (RT). Anti-Hu and anti-Yo positive control sera (Inova Diagnostics Inc.) were also applied at this time for $20 \mathrm{~min}$, at RT. Sera and control antibodies were rinsed off with PBS and sections soaked in PBS for 20 min. Fluorophore-conjugated anti-human secondary antibody was then applied and incubated for $20 \mathrm{~min}$, at RT, followed by rinsing with PBS and further soaking for $20 \mathrm{~min}$. Sections were mounted in glycerol and stored at $4^{\circ} \mathrm{C}$ in the dark until reading. Reagents and detailed methodology are found in Appendix 2.0.

Sections were viewed with a Nikon Eclipse 80i fluorescent microscope (Nikon Instruments, Amstelveen, Netherlands) and scored for anti-Hu / Ri or anti-Yo depending on staining pattern. Positive anti-Hu / Ri, weakly positive or equivocal results were sent for western blot confirmation at the Clinical Immunology Service, University of Birmingham, UK.

\subsubsection{ANTI-CHANNEL ANTIBODY RADIOIMMUNOPRECIPITATION ASSAY}

\subsubsection{Background to methodology}

Over the past 30 years, a number of specific neurotoxins from a variety of vertebrates and invertebrates have been discovered and structurally characterised. In accordance with their amino acid sequence such toxins have differential affinities to channel types and subtypes (Gasparini et al. 1998). These toxins have been an invaluable tool in the pharmacological classification of ion channels (Moczydlowski et al. 1988; Castle et al. 1989; Llinas et al. 1989). In autoimmune neurological disease the detection of circulating autoantibodies has largely depended on the availability of such target-specific 
neurotoxins which may be radiolabelled for use in radioimmunoprecipitation assays e.g. $\partial$-bungarotoxin for the labelling of $\mathrm{nAChRs}$ in the investigation of myasthenia gravis (MG) (Vincent et al. 1985), which are used in immunoprecipitation assays. The principle of radioimmunoprecipitation assays is shown in Figure 2.4.

All serum samples were screened for the following autoantibodies: anti- $\alpha 3$ nAChRs, anti$\mathrm{P} / \mathrm{Q}$ type VGCCs, anti-VGKC, and anti-glutamic acid decarboxylase (GAD). Assays were performed blind to the clinical diagnosis and at least twice by separate investigators.

\subsubsection{Preparation of channel extracts}

Rabbit brain was used for the preparation of VGKC and VGCC channels. Results have previously been shown to be comparable using human and rabbit cerebellar extracts as an antigenic source for potassium (unpublished data), and calcium channels (Motomura et al., 1997). For use in the assay, the membrane preparation was diluted 1:5 in $2 \%$ digitonin solubilisation buffer, incubated for $1 \mathrm{~h}$ at $4{ }^{\circ} \mathrm{C}$ and centrifuged at 13,000 rpm for 15 min. The supernatants were removed, stored at $-70{ }^{\circ} \mathrm{C}$ and used as the source of voltage-gated channels in the assay. Cultured neuroblastoma cells IMR32 were used for the preparation of $\mathrm{nAChR} \alpha 3$ channel subunits. See appendix 3.1 for detailed methodology. 
(a) immunoprecipitation assay

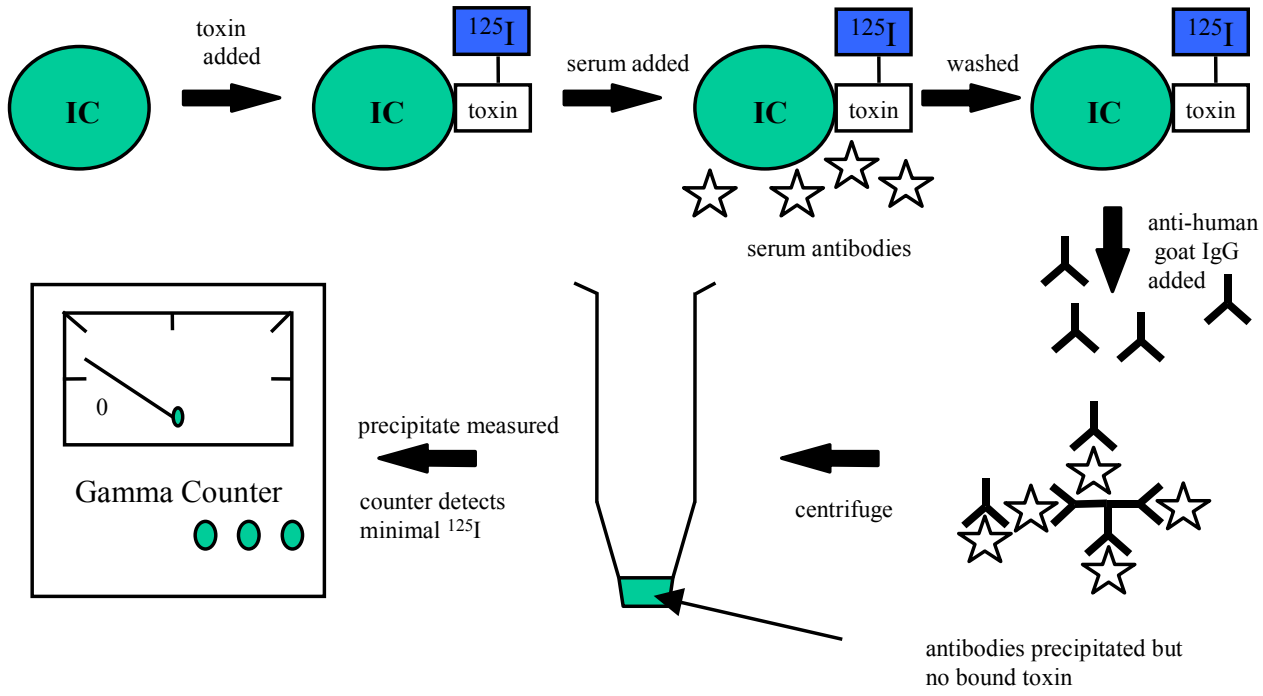

(b) immunoprecipitation assay

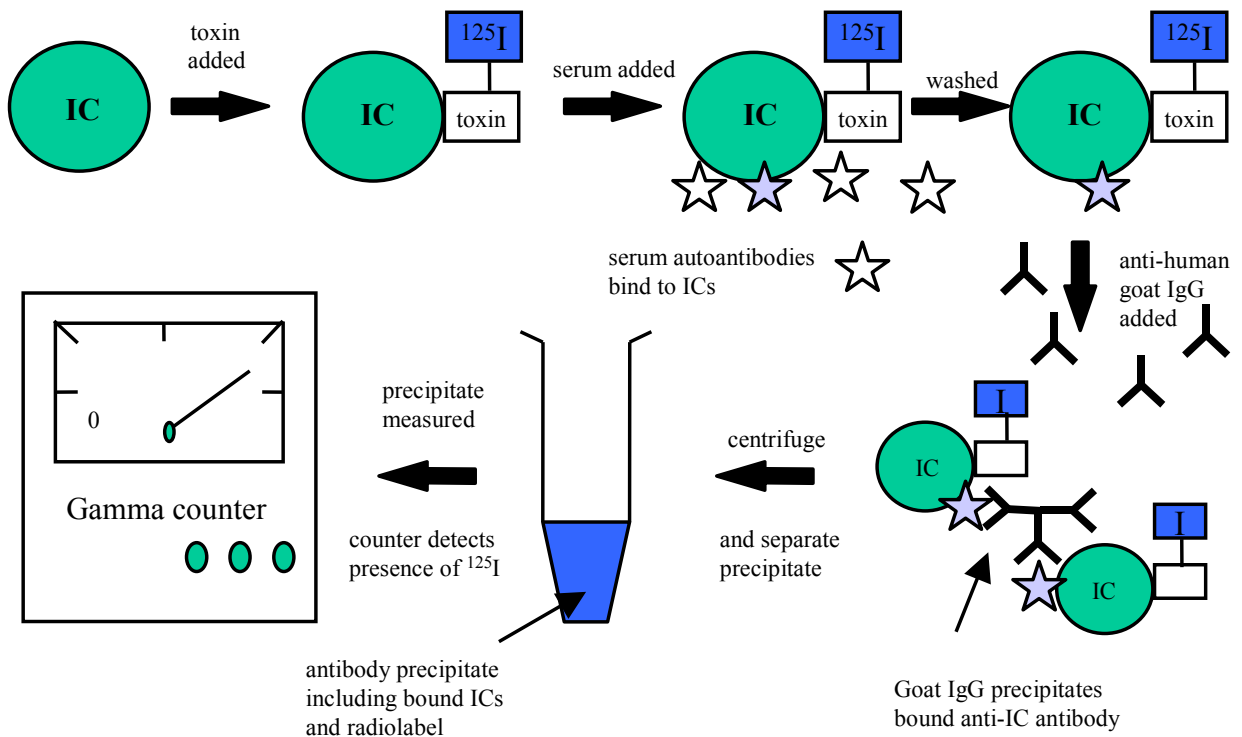

Figure 2.4: Schematic of radioimmunoprecipitation assay. Radiolabelled $\left({ }^{125} \mathrm{I}\right)$ toxin bound to extracted channel preparation is precipitated by anti-human IgG in the presence of antibody (b), but remains in solution when autoantibodies are absent (a). IC = ion channel 


\subsubsection{Immunoprecipitation assays}

1. Sera were tested for antibodies to $\alpha 3$ nAChRs using immunoprecipitation of neuroblastoma nAChRs labelled with ${ }^{125}$ I-Epibatidine $\left({ }^{125} \mathrm{I}\right.$-Epb) (Perkin Elmer, MA, USA).

2. Sera were tested for antibodies to rabbit brain P/Q-type VGCCs using immunoprecipitation of VGCCs extracted from rabbit cerebellum and labelled with ${ }^{125}$ I-labelled- $\omega$-conotoxin MVIIC ( $\left({ }^{125} \mathrm{I}-\omega\right.$-CmTx) (New England Nuclear Ltd. MA, USA).

3. Sera were tested for antibodies to rabbit brain VGKCs using immunoprecipitation of VGKCs extracted from rabbit cerebellum and labelled with ${ }^{125}$ I-labelled- $\alpha$ dendrotoxin $\left({ }^{125} \mathrm{I}-\alpha-\mathrm{DnT} \mathrm{x}\right)$ (Perkin Elmer).

4. Sera were tested for antibodies to glutamic acid decarboxylase (GAD) using a commercially available radioimmunoassay kit (RSR Ltd., Cardiff, UK).

Detailed methodology for channel radiolabelling and immunoprecipitation assays can be found in Appendix 3.2. Briefly, defrosted aliquots of extract were labelled with a saturating (5 fM) concentration of radiolabelled toxin and incubated with diluted sera overnight at $4{ }^{\circ} \mathrm{C}$. To precipitate serum antibodies excess sheep anti-human IgG serum was then added and incubated for 1 hour at RT. The precipitates were pelleted by centrifugation at 13,000 rpm for $5 \mathrm{~min}$, washed in washing buffer $(0.1 \%$ Triton X100 in $20 \mathrm{mM}$ phosphate buffer, $\mathrm{pH}$ 7.4) and counted on an automatic gamma counter (Canberra Packard Cobra II auto-gamma, Meriden ${ }^{\circledR}$, CT, USA). Results were expressed as picomoles of labelled toxin binding sites precipitated per litre of serum after subtraction of non-specific binding (calculated from control mean; see Appendix 3.3 for details). 


\subsubsection{ANTI-M, MUSCARINIC CELL-BASED ASSAY}

\subsubsection{Background to methodology}

This screening assay utilises cultured mammalian cells that have been transiently transfected to express the target autoantigen of interest (Hart et al. 1997). Sera from patients suspected to contain autoantibodies are incubated with transfected cells and any binding revealed using a fluorescently conjugated anti-human secondary antibody. Successful expression of the antigen is verified using a commercial antibody raised against the protein. This assay has a number of advantages over other techniques such as ELISA, immunoprecipitation and other immunohistochemical methods. Firstly, the entire target antigen is translated and expressed in the cell membrane which is closer to in vivo conditions. Secondly, this assay can be used to screen for binding to human antigens as opposed to rodent, which is frequently the tissue used in immunofluorescent screening assays. Thirdly, the antigen is specifically expressed at a high concentration, increasing assay sensitivity. Cell binding assays have been employed to detect autoantibodies in a variety of diseases and have been demonstrated to be more sensitive than traditional immunoprecipitation assays in screening for antibodies in well characterised disorders such as neuromyotonia (Kleopa et al. 2006; Leite et al. 2008).

A number of studies have reported autoantibodies present in Chagas' disease patients with GI disease which in ELISAs bind a synthetic peptide corresponding to a segment of the $\mathrm{M}_{2}$ muscarinic acetylcholine receptor $\left(\mathrm{M}_{2} \mathrm{mAChR}\right)$. We designed and optimised a new cell-based assay to screen for the presence of anti- $\mathrm{M}_{2} \mathrm{mAChR}$ autoantibodies in patients with Chagas' disease using human embryonic kidney cells (HEK 293) transfected to express the $\mathrm{M}_{2} \mathrm{mAChR}$. 


\subsubsection{Bacterial culture}

Escherichia coli (E. coli) DH5a were used for the preparation of plasmid DNA containing the $\mathrm{M}_{2} \mathrm{mAChR}$. All media components were purchased from Oxoid Ltd., Hampshire, UK and sterilised by autoclaving at $121^{\circ} \mathrm{C}, 15$ psi for 20 min. Lysogeny broth (LB; $10 \mathrm{~g} / \mathrm{L}$ tryptone, $5 \mathrm{~g} / \mathrm{L}$ yeast extract, $5 \mathrm{~g} / \mathrm{L}$ sodium chloride) and $\mathrm{L}$ agar were used for the routine growth and maintenance of bacteria. L agar was prepared by the addition of agar $(1.5 \% \mathrm{w} / \mathrm{v})$ to $\mathrm{LB}$ prior to autoclaving. Sterilised $\mathrm{L}$ agar was cooled to 55 ${ }^{\circ} \mathrm{C}$ and heat labile supplements were added (Ampicillin $100 \mu \mathrm{g} / \mathrm{ml}$ ), mixed and poured into $9 \mathrm{~cm}^{2}$ Sterilin ${ }^{\circledR}$ petri dishes (Sterilin Ltd., Caerphilly, UK). DH5 $\alpha$ was maintained on L agar at $4{ }^{\circ} \mathrm{C}$ and subcultured every four to six weeks by overnight growth. Bacterial stocks were stored at $-20{ }^{\circ} \mathrm{C}$ in $0.4 \%$ glucose M9 salts minimal media containing $35 \%$ sterile glycerol (see Appendix 4.1 for details of media compounds and culture methodology).

\section{Growth conditions}

E. coli cultures were grown aerobically at $37{ }^{\circ} \mathrm{C}$ in $250 \mathrm{ml}$ conical flasks, containing 50 $\mathrm{ml}$ of medium with constant shaking at $250 \mathrm{rpm}$ in an orbital shaker. The medium was inoculated with a 1:100 dilution of an overnight $(\sim 16 \mathrm{~h})$ culture generated by seeding 2.5 $\mathrm{ml}$ of medium with a single colony. Growth was monitored hourly by determining the $\mathrm{A}_{650}$ of $100 \mu \mathrm{l}$ or $1 \mathrm{ml}$ samples respectively, or dilutions thereof, using a Unicam Helios $\beta$ UV spectrophotometer (Unicam, Cambridge, UK) (see Appendix 4.1 for details).

\section{Preparation of competent cells}

Once the culture reached an optical density (at $650 \mathrm{~nm}$ ) of $0.4-0.5$, cells were harvested by centrifugation at $4,000 \mathrm{rpm}$ and $4{ }^{\circ} \mathrm{C}$ for $15 \mathrm{~min}$. For volumes of culture less than 1.5 $\mathrm{ml}$, cells were harvested by micro-centrifugation at $13,000 \mathrm{rpm}$ for $5 \mathrm{~min}$ at $4{ }^{\circ} \mathrm{C}$. Cells were re-suspended in $4 \mathrm{ml}$ of ice-cold transformation buffer-I (TFB-I). After incubation 
on ice for $15 \mathrm{~min}$, the cells were harvested and taken up in $2 \mathrm{ml}$ of ice-cold TBF-II (see Appendix 4.1 for TFB compositions). Competent cells were either used within $3 \mathrm{~h}$ or aliquoted $(0.2 \mathrm{ml})$ and stored at $-80^{\circ} \mathrm{C}$.

\subsubsection{Bacterial transformation}

\section{Plasmid}

cDNA for the full length human cholinergic receptor, muscarinic 2 (CHRM2, GenBank NM_001006630) was cloned in-frame with the mammalian cytomegalovirus promoter using the restriction sites EcoRI-XhoI in the polylinker region of pcDNA3 (Invitrogen life technologies, provided by Carl Nelson, University of Leicester). Plasmid DNA was stored at $-20^{\circ} \mathrm{C}$ in ultrapure (Promega UK Ltd., Southampton, UK).

\section{Transformation}

Various dilutions of plasmid DNA (1-50 ng in $4 \mu \mathrm{l}$ ) were added to $0.2 \mathrm{ml}$ aliquots of competent cells, gently mixed, and incubated on ice for $40 \mathrm{~min}$, followed by heat-shock at $42{ }^{\circ} \mathrm{C}$ for $1 \mathrm{~min}$ to facilitate uptake of the DNA. After cooling on ice for $5 \mathrm{~min}, 0.7 \mathrm{ml}$ of pre-warmed LB was added to the mixture and incubated at $37{ }^{\circ} \mathrm{C}$ for $30-60 \mathrm{~min}$ to allow the expression of the antibiotic resistance genes. Finally the cells were harvested by centrifugation at 13,000 rpm for $5 \mathrm{~min}$ and $0.7 \mathrm{ml}$ of supernatant removed. The cells were re-suspended in the remaining supernatant and plated out on $\mathrm{L}$ agar plates containing ampicillin and incubated at $37{ }^{\circ} \mathrm{C}$ overnight (see Appendix 4.2 for detailed methodology). 


\subsubsection{Plasmid DNA isolation}

\section{Plasmid DNA Miniprep preparations}

The QIAprep ${ }^{\circledR}$ Minipreps DNA Purification Kit (Qiagen Ltd., West Sussex, UK) was used according to the centrifugation protocol supplied by the manufacturer for plasmid DNA yields of less than $10 \mu \mathrm{g}$. Depending on the copy number of the plasmid, $1-5 \mathrm{ml}$ (high-copy number plasmids) or $10 \mathrm{ml}$ (low-copy number plasmids) overnight cultures were used (see Appendix 4.3 for detailed methodology).

\section{Plasmid DNA Maxiprep preparations}

For up to $850 \mu \mathrm{g}$ plasmid DNA, a $200 \mathrm{ml}$ overnight culture was manipulated using the PureLink $^{\mathrm{TM}}$ HiPure Plasmid (Maxi) Filter Purification Kit (Invitrogen Ltd., Paisley, UK) according to the manufacturer's instructions. The DNA pellet was re-suspended in 200$500 \mu 1 \mathrm{TE}$ Buffer (in kit) and used in downstream applications or stored at $-20{ }^{\circ} \mathrm{C}$ (see Appendix 4.3 for detailed methodology).

\subsubsection{DNA sequencing}

\section{DNA digestion with restriction endonucleases}

To confirm the presence and check the correct orientation of the CHRM2 insert, the purified plasmid was analysed by DNA digestion. DNA digestion was performed using restriction enzymes (EcoR1 and Xho1) according to the manufacturer's instructions (New England BioLabs UK Ltd., Hertfordshire, UK). Approximately 5 units of enzyme per $\mu \mathrm{g}$ of DNA were incubated at $37^{\circ} \mathrm{C}$ for $1-3 \mathrm{~h}$ (see Appendix 4.3 for detailed methodology).

\section{Agarose gel electrophoresis}

Agarose gel electrophoresis was used to separate and visualise DNA fragments, and was performed in a Bio-Rad Mini/Wide-Sub cell (Bio-Rad Labs. Ltd. Hertfordshire, UK). 
Agarose gels were $0.7-1.5 \%$, depending on the size of the DNA fragments, in $0.5 \mathrm{x}$ TBE buffer (see Appendix 4.3 for composition), containing $0.5 \mu \mathrm{g} / \mathrm{ml}$ ethidium bromide. Samples were prepared by the addition of 6x loading buffer (Appendix 4.3) and electrophoresed in gels submerged in $0.5 \mathrm{x}$ TBE buffer for $45-120 \mathrm{~min}$ at $70-100 \mathrm{~V}$ (constant). The size marker, Hyperladder I (Bioline, London, UK) was loaded alongside samples for estimating both the size and quantity of DNA following gel electrophoresis. After electrophoresis the DNA bands were visualised using a 2011 Macrovue UV transilluminator (LKB Instruments, Victoria, Australia) (see Appendix 4.3 for solutions and detailed methodology).

\section{DNA sequencing}

Final validation was achieved by sequencing the CHRM2 insert from the purified plasmid DNA to confirm that the gene was in frame. DNA sequencing was performed by the in-house service at the Weatherall Institute of Molecular Medicine (WIMM) (John Radcliffe Hospital, Oxford, UK) on a 48 capillary ABI-3730 DNA cycle sequencer (Applied Biosystems, California, USA). Template plasmid DNA ( $>10 \mathrm{ng}$ depending on the size of the insert being sequenced) was provided in solution to the WIMM sequencing service. Sequencing reactions were performed by using the T7 forward primer: 5'-TAA TAC GAC TCA CTA TAG GG-3' (3.2 pmol/ $\mu$ l) and for the reverse sequence the Sp6 reverse primer: 5'-ATT-TAG-GTG-ACA-CTA-TAG-3' (3.2 pmol/ $\mu \mathrm{l})$.

\subsubsection{Mammalian cell culture}

Human embryonic kidney (HEK293) cells

HEK 293 cells were grown in cell culture flasks containing Dulbecco's modified Eagles Medium (DMEM) (Sigma-Aldrich, Dorset, UK) containing fetal calf serum (FCS) (Sigma-Aldrich) and antibiotics (PSA: penicillin, amphotericin and streptomycin) (Invitrogen, Paisley, UK) (see Appendix 4.4 for compositions) and split at a ratio of 1:8 
every three days. On day 3 cells were harvested following removal of DMEM by aspiration, washing in PBS and trypsinising for $1 \mathrm{~min}$ at $37^{\circ} \mathrm{C}$. Cells were re-suspended in DMEM containing FCS and PSA, $10 \mu 1$ extracted, added to an equal amount of Trypan Blue (Sigma-Aldrich) and counted on a haemocytometer. The remaining cell suspension was centrifuged at $1000 \mathrm{rpm}$ for $6 \mathrm{~min}$. After removing the supernatant the remaining cell pellet was re-suspended in DMEM containing FCS and PSA. A volume containing $1.8 \mathrm{x}$ $10^{6}$ cells was added to DMEM containing FCS and PSA to make a final volume of $12 \mathrm{ml}$. The remaining suspension was split 1:8 and placed in a new flask for further growth.

\section{Transfection}

Cells were seeded onto poly-L-lysine coated cover-slips in 6-well tissue culture plates (3 coverslips / well). $2 \mathrm{ml}$ ( $3 \times 10^{5}$ cells) of cell suspension were added to each well. Cells were maintained in growth medium overnight at $37{ }^{\circ} \mathrm{C}$. Medium was changed to antibiotic-free DMEM and cells were transfected with the CHRM2-containing plasmid using the transfection agent polyethylenimine (PEI) (Sigma-Aldrich). This was subsequently changed to lipofectamine (Invitrogen) to reduce background staining in the immunofluorescence assay. Cells were incubated for $24 \mathrm{~h}$ at $37{ }^{\circ} \mathrm{C}$ with antibiotic-free DMEM containing per well: $4 \mu \mathrm{g}$ DNA and $10 \mu \mathrm{l}$ lipofectamine. Some cells were transfected with plasmids containing green fluorescent protein (GFP) (AB Vector, San Diego, CA, USA) as a transfection control (see Appendix 4.4 for detailed methodology).

\section{Indirect immunofluorescence}

The following day the medium was changed and cells were maintained for a further $24 \mathrm{~h}$ at $37^{\circ} \mathrm{C}$. Cover-slips were then transferred to 24-well culture plates washed with PBS and incubated with Chagas' disease or community control serum diluted 1:20 in DMEM containing HEPES and 1\% BSA for $1 \mathrm{~h}$ at RT. Cells stained with a monoclonal antibody against $\mathrm{M}_{2} \mathrm{mAChR}$ (1:200) (Abcam, Cambridgeshire, UK) served as a positive control. As this antibody is raised against an intracellular epitope, these cells were first fixed with 
4\% formaldehyde for $10 \mathrm{~min}$ and permeabilised with $1 \%$ Triton $\mathrm{X}$. Cells were washed three times in DMEM containing HEPES (DMEM wash) and incubated with a fluorescently-conjugated secondary antibody (anti-human for cells stained with serum, anti-mouse for control cells) (Invitrogen Ltd. Paisley, UK) diluted 1:700 for $45 \mathrm{~min}$ in the dark at RT. Cells were washed again in several changes of DMEM wash and PBS and mounted on microscope slides using fluorescent mounting medium (Dako, Cambridgeshire, UK) containing the fluorescent DNA stain 4',6-diamidino-2phenylindole (DAPI; Roche Diagnostics Ltd. Burgess Hill, West Sussex, UK). Slides were refrigerated at $4^{\circ} \mathrm{C}$ in the dark and allowed to dry over night before reading (see Appendix 4.4 for detailed methodology).

\subsection{FUNCTIONAL IN VITRO EFFECTS OF CHAGASIC SERA}

\subsubsection{IN VITRO CONTRACTILITY EXPERIMENTS}

\section{Background to methodology}

Evoking changes in smooth muscle contractility by electrical field stimulation (EFS) is an established tool for the study of contractility (Bulbring et al. 1967; Kosterlitz et al. 1970). This technique involves maintaining tissue in a physiological solution between platinum ring electrodes and applying pulses of electricity across the muscle to induce a response. Relaxations and contractions are measured by way of an isometric force-transducer to which the muscle is attached and kept under a baseline muscle tension. The muscle is also kept at a constant resting, baseline tension (Figure 2.5). The initial response to EFS may be a contraction or relaxation, depending on whether excitatory or inhibitory neurons form the majority of neurons stimulated. This initial response is usually followed by an after contraction. The pharmacology of the smooth muscle responses may be investigated by adding compounds to the organ bath solution and measuring changes to contractility. The advantages of EFS over simple contractility studies using only a resting 
tension is that EFS enables neurogenic responses (which occur during EFS) and myogenic responses to be distinguished.

\section{Chagas' disease and control sera}

The effect of sera from three patients with chagasic colonic dysfunction, three with Chagas' disease and no GI sequalae and three healthy community controls on human colonic smooth muscle responses to EFS were investigated as previous studies have shown that chagasic sera may increase the tone of resting smooth muscle.

\section{Human tissue}

Resection margin segments of human colon (ascending, transverse or sigmoid) were obtained from patients undergoing surgery for cancer at the Royal London Hospital. The segments were transferred to the research laboratories within $2 \mathrm{~h}$ after resection in icecold Kreb's solution (containing in mmol L): $\mathrm{NaCl} 121.5, \mathrm{CaCl}_{2} 2.5, \mathrm{KH}_{2} \mathrm{PO}_{4} 1.2, \mathrm{KCl}$ 4.7, $\mathrm{MgSO}_{4} 1.2, \mathrm{NaHCO}_{3} 25$, glucose 5.6) equilibrated with $5 \% \mathrm{CO}_{2}$ and $95 \% \mathrm{O}_{2}$. The segments were opened and the mucosa removed by blunt dissection and discarded. Strips $(3-5 \times 15 \mathrm{~mm})$ from the inter-taenial parts of the segment were cut parallel to the circular muscle fibres. These strips were used immediately or stored overnight at $4{ }^{\circ} \mathrm{C}$ in fresh, oxygenated Kreb's solution before use. 


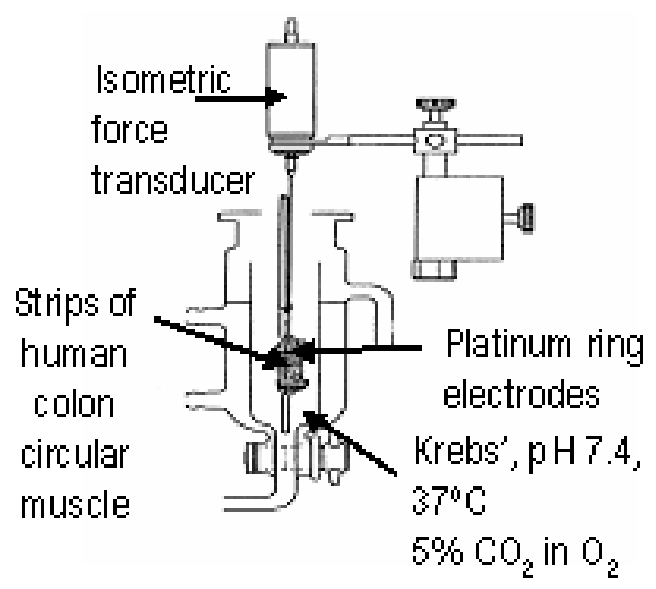

Figure 2.5: Organ bath for electrical field stimulation and measurement of smooth muscle contractility. Strips of human colonic smooth muscle maintained in a physiological solution were placed between platinum ring electrodes and electrically stimulated. Smooth muscle contractions were measured by an isometric force transducer (Courtesy of $\operatorname{Dr} \mathrm{J}$ Broad, Wingate Inst. of Neurogastroenterology, London).

In vitro contractility studies

The strips ( $\mathrm{n}=4-16$ from each patient) were mounted in tissue baths containing $5 \mathrm{ml}$ Kreb's solution at $37{ }^{\circ} \mathrm{C}$ and gassed with $5 \% \mathrm{CO}_{2}$ in $\mathrm{O}_{2}$. The change in the tension was recorded using isometric force transducers (AD Instruments, Chalgrove, UK) on a data acquisition system (Biopac Systems Inc., Goleta, CA, USA). The strips were given $2 \mathrm{~g}$ tensions and allowed to equilibrate for 45 min during which time the bath solutions were changed every $15 \mathrm{~min}$. At the end of the equilibration period the strips were stimulated via two parallel platinum ring electrodes connected to a stimulator (STG2008, Scientifica, Uckfield, UK). The stimulation parameters were $50 \mathrm{~V}(200 \mathrm{~mA}), 5 \mathrm{~Hz}, 0.5$ ms bipolar pulse duration, for $10 \mathrm{~s}$, every $1 \mathrm{~min}$. These EFS parameters in preliminary experiments were shown to elicit sub-maximal contractions. The effects of sera from patients with Chagas' disease were investigated by adding serum direct to the organ bath, using a single concentration (1\%). The effects of control sera from healthy community 
controls were also measured in each experiment. The effect of the serum in a single strip was determined from at least three EFS-induced responses. The effect of chagasic sera on cholinergic and nitrergic pathways were investigated by pre-incubating strips with atropine (a cholinergic antagonist) or N- $\omega$-nitro-L-arginine methyl ester (L-NAME; a nitric oxide synthase inhibitor) respectively. Serum effects were also determined following tissue pre-incubation with the voltage-gated sodium channel blocker tetrodotoxin (TTX). Minimum contact time for pharmacological agent was $30 \mathrm{~min}$.

\subsection{EXPRESSION AND LOCALISATION OF ENTERIC KV1 CHANNELS IN THE HUMAN GI TRACT}

\subsubsection{INDIRECT IMMUNOHISTOCHEMISTRY}

\section{Background to methodology}

Immunohistochemistry utilises labelled antibodies as reagents for the optical detection and localisation of antigenic material (usually proteins) in cells and tissues (Coons et al. 1950). As such, this method is dependent on the specificity of antibody binding to the chosen epitope. Immunohistochemistry involves a number of steps beginning with the 'unmasking' of antigens which may be hidden by the fixation process (e.g. formalin cross-links) with antigen retrieval. A number of techniques such as pressure cooking, protease treatment, and microwaving in an appropriate buffer may be employed, although the molecular mechanisms underlying the process are poorly understood (Shi et al. 2001). Following antigen retrieval, the specific primary antibody is applied and incubated with the tissue before washing to remove excess. A species-specific secondary antibody which is conjugated to a tag such as biotin or a fluorophore is applied which binds to the primary antibody. Finally, if required, a detection reagent containing a chromagen is applied that binds to the secondary conjugate and allows visualisation of the antibody complex (e.g. avidin-biotin). Primary antibody specificity should be checked using 
control tissue that is known to contain the antigen, and ideally by immunoblot. Negative controls, where the primary antibody is omitted, should also be included in each experiment (Burry 2000).

The composition of all solutions and reagents are detailed in Appendix 5.1.

\section{Clinical material}

Sections from formalin-fixed, paraffin wax-embedded tissue samples of disease-free, full thickness human oesophagus, stomach, jejunum, ileum and colon were used in this study. Stomach, small intestinal and colonic tissues were identified and retrieved from the Archive of Pathology at the Royal London Hospital (London, UK). Due to a lack of archived specimens, oesophageal tissue was obtained prospectively (full thickness surplus tissue at oesophageal cancer resections) and processed in the same manner as archived tissue (see Appendix 5.2 for routine tissue processing and section preparation). Prior to immunostaining, a $3 \mu \mathrm{m}$ section from each tissue block was cut, collected on coated slides (VWR International, Leicestershire, UK) and stained with haematoxylin and eosin (H\&E) (Appendix 5.3). These sections were then inspected using light microscopy to confirm that there were no inflammatory or other histological abnormalities. For each anatomical location studied, anti-channel immunostaining was performed on a minimum of five tissue samples, from different patients.

\section{Control tissue}

Formalin-fixed, paraffin wax-embedded murine (strain: C57 black 6) cerebella were obtained from the Blizzard Institute of Cell and Molecular Science (ICMS) Pathology Group, (Queen Mary University, London, UK) control tissue bank. KV1 channel distribution has been previously well-described in this brain region (Veh et al. 1995; Chung et al. 2001) 


\section{Indirect immunohistochemistry}

Indirect immunohistochemical staining was performed using a kit-based (Vectastain Universal Elite ABC: Vector Laboratories, Burlingame, CA, USA) avidin-biotinperoxidase complex method (see Appendix 5.4 for detailed methodology) with commercially available antibodies raised against $\mathrm{K}_{\mathrm{V}} 1$ channel $\alpha$-subunits (Santa Cruz Biotechnology Inc, CA, USA). Tissue sections were de-waxed in two changes of xylene for 2 min and dehydrated in two changes of industrial methylated spirit (IMS) for $2 \mathrm{~min}$. After washing in running tap water for 5 min thermal antigen retrieval was carried out by microwaving sections in a citrate buffer (Vector Laboratories, Burlingame, CA, USA) for $25 \mathrm{~min}$. Sections were allowed to cool for $5 \mathrm{~min}$ and washed again in running tap water for $5 \mathrm{~min}$. Endogenous peroxidase activity was blocked by placing sections in methanol containing $3 \% \mathrm{H}_{2} \mathrm{O}_{2}$ for $15 \mathrm{~min}$. After further washing in running tap water for $5 \mathrm{~min}$ and soaking in wash buffer (WB; Dako, Cambs, UK) for 2 min, sections were then incubated in normal horse serum (in kit) for $20 \mathrm{~min}$ at RT. Excess blocking serum was tipped off and sections were incubated with primary anti-human $\mathrm{K}_{\mathrm{V}} 1$ antibodies raised in goat diluted in antibody diluent (Dako, Cambs, UK) for $60 \mathrm{~min}$ at RT (see Table 2.5 for dilutions). Sections incubated with antibody diluent only served as negative controls. Primary antibodies were washed off with WB and sections incubated with secondary anti-goat biotinylated antibodies raised in horse (Vector Laboratories, Burlingame, CA, USA) diluted 1:250 in WB, for 30 min at RT. Secondary antibody was washed off with WB and sections incubated in avidin complex solution (in kit) for $30 \mathrm{~min}$ at RT, washed again with WB and incubated in diaminobenzidine solution (Bio-Genex-Laboratories, San Ramon, CA, USA) for 5 min. After further washing in running tap water for 5 min, sections were counter-stained in Gill's haematoxylin for 2 min, washed in running tap water for $5 \mathrm{~min}$, dehydrated twice in IMS for $2 \mathrm{~min}$, cleared twice in xylene for $2 \mathrm{~min}$ and mounted in Canada balsam (VWR International, Leicestershire, UK). 


\section{Light microscopy and qualitative analysis}

Sections were analysed at magnifications of $\mathrm{x} 10, \mathrm{x} 20$ and $\mathrm{x} 40$ using a light microscope (Leitz Dialux 20, Leica Microsystems UK Ltd., Milton Keynes, UK). The presence and distribution of anti-channel immunostaining was noted and a representative area for each gut region was photographed with a digital camera using the Leica IM50 Image Manager software (Leica Microsystems, UK).

Table 2.6: Anti-K 1 channel antibody dilutions used for indirect immunohistochemistry

\begin{tabular}{cc}
\hline Primary antibody & Dilution \\
\hline $\mathrm{K}_{\mathrm{V}} 1.1$ & $1: 150$ \\
$\mathrm{~K}_{\mathrm{V}} 1.2$ & $1: 75$ \\
$\mathrm{~K}_{\mathrm{V}} 1.3$ & $1: 75^{*}$ \\
$\mathrm{~K}_{\mathrm{V}} 1.4$ & $1: 75$ \\
$\mathrm{~K}_{\mathrm{V}} 1.5$ & $1: 75$ \\
$\mathrm{~K}_{\mathrm{V}} 1.6$ & $1: 75$ \\
\hline
\end{tabular}

All antibodies incubated for one hour at room temperature, except $\mathrm{K}_{\mathrm{V}} 1.3^{*}$ which was incubated overnight at $4^{\circ} \mathrm{C}$

\subsubsection{CHARACTERISATION OF ENTERIC NEURONAL KVI.4 LOCALISATION}

The distribution of $\mathrm{K}_{\mathrm{V}} 1.4$ immunostaining in enteric ganglia was distinct from other subunits (see Results sections 3.12-3.14). A similar distribution of $\mathrm{K}_{\mathrm{V}} 1.4$ containing channels has previously been demonstrated in excitatory neurons of the hippocampal formation in the brain and was localised to axonal segments and pre-synaptic termini (Cooper et al. 1998). We therefore investigated whether $\mathrm{K}_{\mathrm{V}} 1.4$ is specifically localised to these areas in enteric neurons with indirect immunofluorescent double-staining employing antibodies raised against two corresponding neuronal peptides: neurofilament 
(NF) (Diepholder et al. 1991; Luider et al. 1992) and synaptophysin (Wiedenmann et al. 1986; Stefaneanu et al. 1988). Neurofilaments belong to the intermediate filament family of proteins and are found specifically in neurons where they form a key component of the axonal and dendritic cytoskeleton (Davison et al. 1960; Schlaepfer 1987). Synaptophysin is a glycoprotein present in the membrane of pre-synaptic vesicles (Wiedenmann et al. 1985).

\section{Clinical material}

Sections from formalin-fixed, paraffin wax-embedded tissue samples of disease-free, full thickness stomach were used in this study. As in the previous immunohistochemical study, tissue was obtained from the Archive of Pathology at the Royal London Hospital (Whitechapel, London, UK). This region was chosen due to tissue availability and high levels of $\mathrm{K}_{\mathrm{V}} 1.4$ subunits exhibiting this distribution.

\section{Indirect immunofluorescence}

Tissue sections were de-waxed in two changes of xylene for 2 min and dehydrated in two changes of IMS for $2 \mathrm{~min}$. After washing in running tap water for $2 \mathrm{~min}$, thermal antigen retrieval was carried out by microwaving sections in a citrate buffer (Vector Laboratories, Peterborough, UK) for $18 \mathrm{~min}$. Sections were allowed to cool for $5 \mathrm{~min}$, were washed again in running tap water for 2 min and then incubated with a serum-free protein block (Dako, Cambs, UK) for 10 min at RT. Excess protein block was tipped off and sections were incubated with a mixture of anti- $K_{V} 1.4$ and an anti-neuronal primary antibody diluted in PBS for $24 \mathrm{~h}$ at $4{ }^{\circ} \mathrm{C}$ (see Table 2.6 for primary antibody details and dilutions). After allowing sections to reach room temperature primary antibodies were washed off by soaking in two changes of PBS for 4 min and incubated with a mixture of appropriate Alexa Fluor ${ }^{\circledR}$ fluorescently-conjugated secondary antibodies (Invitrogen Ltd. Paisley, UK) in the dark for 45 min at RT. Secondary antibodies were washed off in two changes of PBS for 4 min and sections were mounted in fluorescence mounting medium (Dako, 
Ely, Cambridgeshire, UK) containing DAPI (1:1000) for nuclear visualisation (see Appendix 5.5 for detailed methodology).

Table 2.7: Antibodies used in indirect immunofluorescence

\begin{tabular}{|c|c|c|c|}
\hline Primary antibody & Source & Dilution & Secondary antibody \\
\hline $\mathrm{K}_{\mathrm{V}} 1.4$ & $\begin{array}{l}\text { Goat polyclonal against internal } \\
\text { region of human peptide }\end{array}$ & $1: 25$ & $\begin{array}{l}\text { Alexa fluor (568) } \\
\text { donkey anti-goat }\end{array}$ \\
\hline Synaptophysin & $\begin{array}{l}\text { Mouse monoclonal against coated } \\
\text { vesicles from bovine brain }\end{array}$ & $1: 100$ & $\begin{array}{l}\text { Alexa fluor (488) } \\
\text { donkey anti-mouse }\end{array}$ \\
\hline NF-2F11 & $\begin{array}{l}\text { Mouse monoclonal against } \\
\text { isolated neurofilament from } \\
\text { human brain }\end{array}$ & $1: 100$ & $\begin{array}{l}\text { Alexa fluor (488) } \\
\text { donkey anti-mouse }\end{array}$ \\
\hline
\end{tabular}

Primary antibodies were incubated for $1 \mathrm{~h}$ at RT. All secondary antibodies were used at dilution of 1:200 and incubated for 45 min at RT. NF-2F11 = neurofilament, clone $2 \mathrm{~F} 11$.

Fluorescent microscopy and qualitative analysis

Sections were viewed with a multi-channel fluorescence microscope (Olympus BX61UCB, Olympus UK Ltd., Essex, UK). Sequential images were captured (Cell* imaging software, Soft Imaging Solutions GmbH, Münster, Germany) and overlaid using Adobe Photoshop (Version 7.0, Adobe Systems Inc., San Jose, CA, USA). 


\section{RESULTS}

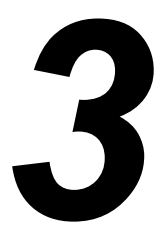

\subsection{DETECTION OF AUTOANTIBODIES}

\subsubsection{ANTI-NEURONAL ANTIBODY ASSAY}

Twenty seven samples from patients with severe intestinal dysmotility (SID) were screened for the presence of anti-neuronal antibodies, the presence of which may be indicative of a paraneoplastic process. An indirect immunofluorescence assay using monkey cerebellum as substrate was employed to determine the presence of anti-neuronal nuclear (anti-Hu / Ri), or anti-Purkinje cell cytoplasm antibodies (anti-Yo). Commercial anti-Hu and anti-Yo antibodies were used as positive staining controls. Pooled normal serum was used as negative control. Samples which produced positive nuclear staining were sent for western blot confirmation of antigen specificity, as this assay cannot distinguish between anti-Hu and $\mathrm{Ri}$ antibodies.

Anti-Hu antibodies produced strong nuclear staining of cells from all three cerebellar layers (granular, purkinje and molecular). Anti-Yo staining was clearly distinguishable from anti-Hu, revealing punctate staining of Purkinje cell cytoplasm only. PNS failed to stain any cerebellar structures. Three samples from patients with SID (1 chronic intestinal pseudo-obstruction and 2 enteric dysmotility) moderately stained cell nuclei of the granular, purkinje and molecular layers and were sent for western blot confirmation. The 
remaining 24 samples produced no staining. The three samples sent for confirmation were found to be negative (Figure 3.1). These negative findings minimise the likelihood that these cases of SID had a paraneoplastic basis.
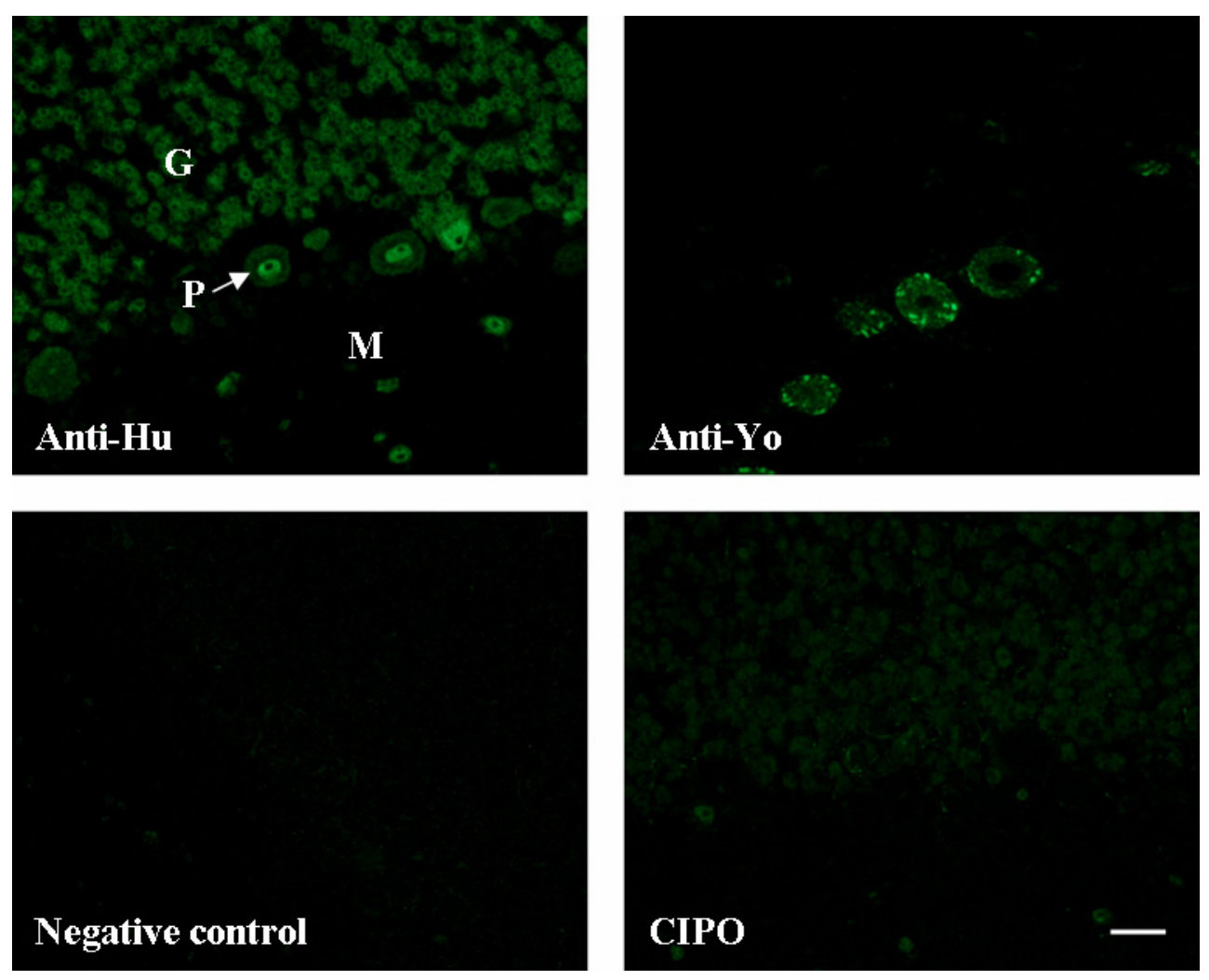

Figure 3.1: Cerebellar anti-neuronal immunostaining. Sections of cerebellum were used as substrate to screen for the presence of anti-neuronal autoantibodies. Anti-Hu positive staining control stained nuclei of granular layer cells, Purkinje cells (arrow) and cells of the molecular layer. Anti-Yo positive staining control specifically stained puncta in Purkinje cell cytoplasm only. Pooled normal serum served as the negative control and failed to stain any cerebellar cells. Sera from two patients with chronic intestinal pseudo-obstruction and 1 with enteric dysmotility moderately stained cells of all three layers and required western blot confirmation. An example from one of these cases is shown in the lower right panel. All three cases were subsequently found to be negative. $\mathrm{G}=$ granular layer, $\mathrm{P}=$ Purkinje cell, $\mathrm{M}=$ molecular layer, $\mathrm{CIPO}=$ chronic intestinal pseudo-obstruction. Scale bar $=30 \mu \mathrm{m}$. 


\subsubsection{ANTI-CHANNEL ANTIBODY RADIOIMMUNOPRECIPITATION ASSAYS}

Sera from all patient groups (21 achalasia, 27 SID and 61 Chagas' disease, 20 Brazilian healthy control and 10 UK healthy controls) were screened for the presence of antichannel antibodies using radioimmunoprecipitation assays. In each assay, with the exception of the glutamic acid decarboxylase assay, where it is considered only necessary to repeat positive results (Powell et al. 1996) each sample was tested twice by different investigators blinded to the diagnosis, to confirm the result. Due to limited quantities of chagasic serum samples, it was not possible to screen the full complement of 61 samples in every assay.

\subsubsection{Ganglionic nicotinic acetylcholine receptor: alpha 3 subunit}

Assays performed twice showed good agreement between results: mean difference between assays $=4 \mathrm{pM}(95 \% \mathrm{CI}-2$ to 9$), \mathrm{p}=0.0007$. All positive and negative control sera were positive and negative in the expected titre ranges. None of the GINMD, Chagas' disease or control sera were found to be positive (Figure 3.2).

\subsubsection{Glutamic acid decarboxylase}

Three sera from Brazil showed precipitation of the complex which was comparable to the 'low positive' calibration control. Two such low positive samples were from patients with Chagas' disease, one with cardiac complications the other with colonic disease, and the third from a healthy community control. A low positive result would suggest that these individuals are predisposed to or have Type I diabetes mellitus. However, these titres are not considered high enough to have a neurological impact (Saiz et al. 2008; Liimatainen et al. 2010). None of the GINMD or UK healthy control sera were positive Figure 3.3). 


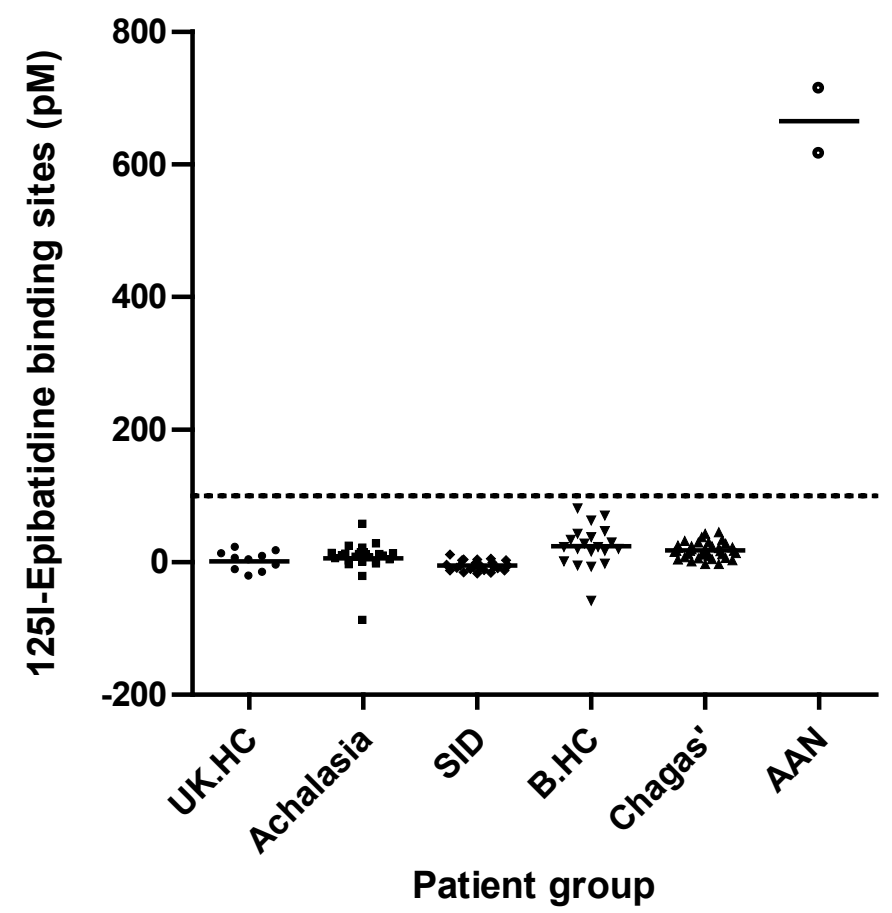

Figure 3.2: Scatter plot of results from anti- $\alpha 3 \mathrm{nAChR}$ immunoprecipitation assay.

None of the samples from any patient group were positive for anti- $\alpha 3$ antibodies. UK.HC $=$ UK healthy controls, $\mathrm{SID}=$ severe intestinal dysmotility, $\mathrm{B} \cdot \mathrm{HC}=$ Brazilian healthy controls, $\mathrm{AAN}=$ autoimmune autonomic neuropathy. Dotted line indicates 100pM, above which samples were regarded as positive. 


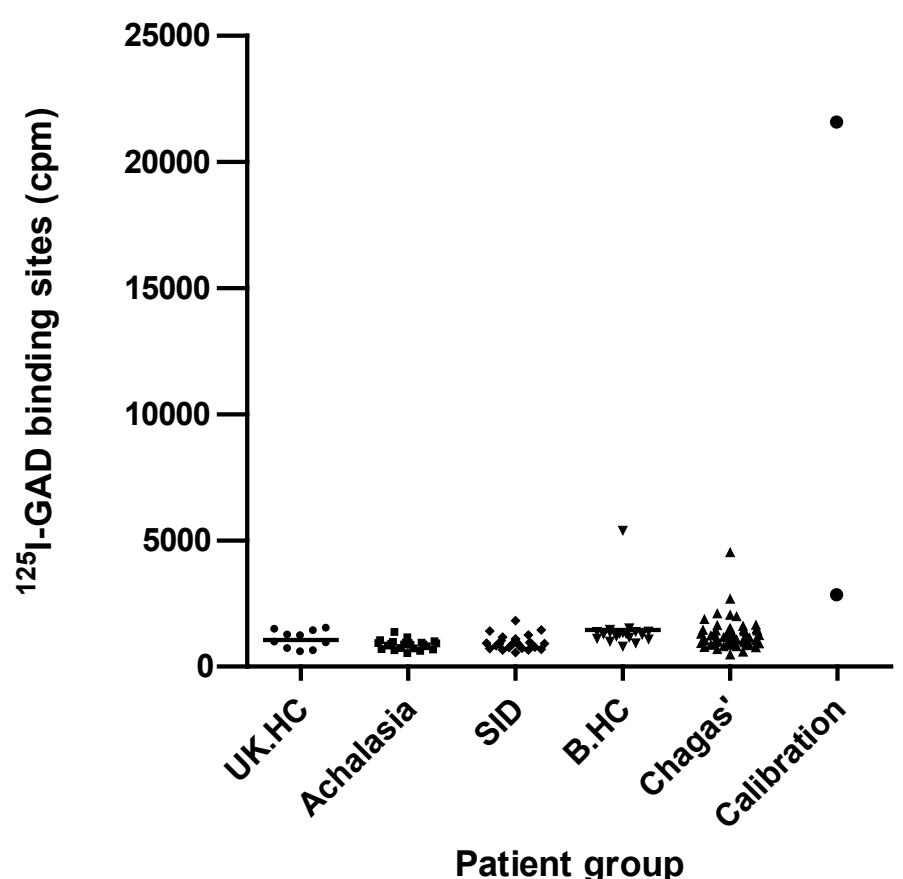

Figure 3.3: Scatter plot of results from anti-GAD immunoprecipitation assay. Two Chagas' disease samples and one Brazilian healthy control were weakly positive for anti-GAD antibodies. $\mathrm{UK} \cdot \mathrm{HC}=\mathrm{UK}$ healthy controls, SID $=$ severe intestinal dysmotility B.HC $=$ Brazilian healthy controls.

\subsubsection{Voltage-gated calcium channels (VGCCs)}

One of 57 Chagas' disease patients showed strong precipitation of the labelled channel complex in a range comparable to LEMS positive controls (246 pM of MVIIC binding sites per litre of serum). [LEMS median: 194, range 131 to $256 \mathrm{pM}$ ]. At the time of venepuncture this patient had not received anti-T.cruzi therapy and did not have secondary manifestations of Chagas' disease. No GINMD patient sample or control sample was positive (Figure 3.4). 


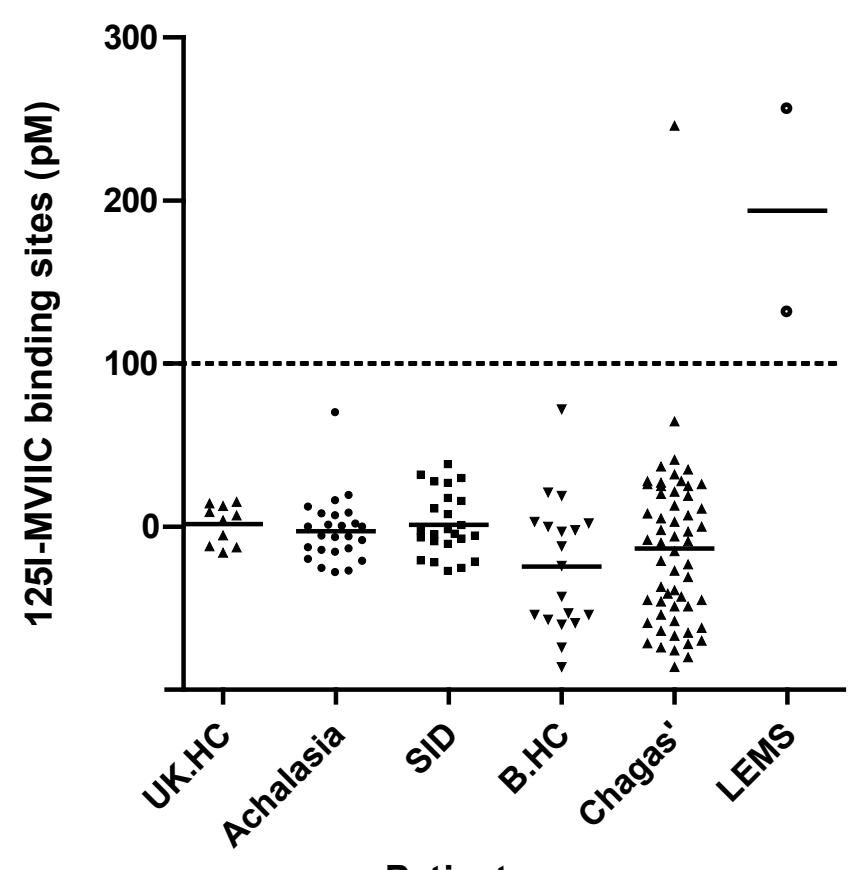

Patient group

Figure 3.4: Scatter plot of results from anti-VGCC immunoprecipitation assay. One Chagas' disease serum sample was strongly positive for anti-P/Q type VGCC antibodies. All other samples were negative. LEMS positive controls were positive in the expected titre ranges. UK.HC $=$ UK healthy controls, SID $=$ severe intestinal dysmotility, B.HC $=$ Brazilian healthy controls, LEMS $=$ Lambert-Eaton myasthenic syndrome.

\subsubsection{Voltage-gated potassium channels (VGKCs)}

Assay repeats showed very high agreement: mean difference between assays $=12 \mathrm{pM}$ (95\% CI -4 to 28), $\mathrm{p}<0.0001$. Eight of 59 sera from patients with Chagas' disease showed precipitation of the labelled complex that was greater than $100 \mathrm{pM}$ of binding sites per litre of serum. The eight positives did not however define a specific clinical phenotype: 5 were female, 5 had no heart or gut manifestations, one had achalasia and heart disease, one had achalasia only and another had colonic dysfunction only. [Whole Chagas' disease group: median 34, range -16 to 282]. Although consistently positive, 
none of the eight were in a range comparable to NMT positive controls [Chagas' disease positive median: NMT median: 1199, range 776 to $1622 \mathrm{pM}$ ). All of the samples from patients with primary GINMD were found to be negative as were all UK and Brazilian healthy controls (Figure 3.5).

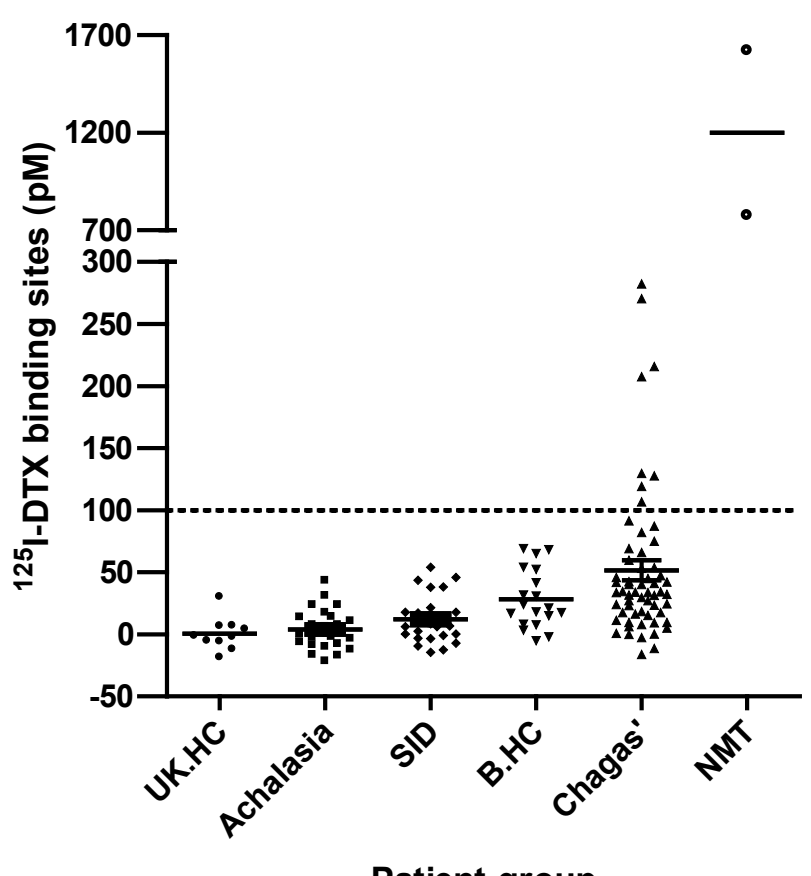

Figure 3.5: Scatter plot of results from anti-VGKC immunoprecipitation assay. Eight Chagas' disease sera were weak to moderately positive for anti-VGKC antibodies. None, however, were in the same range as the NMT positive controls. UK.HC = UK healthy controls, SID $=$ severe intestinal dysmotility, B.HC = Brazilian healthy controls, NMT = neuromyotonia. 


\subsubsection{ANTI-M2 MUSCARINIC ACETYLCHOLINE RECEPTOR CELL-BASED ASSAY}

\subsubsection{Anti-M $\mathrm{M}_{2}$ muscarinic acetylcholine receptor assay optimisation}

Cell transfection efficiency was measured by green fluorescent protein (GFP) transfection where protein expression results in cells fluorescing green. This was performed in several assays and was judged as good by the fact the majority of cells in several microscopy views were positive (Figure 3.6). The success of $\mathrm{M}_{2}$ muscarinic acetylcholine receptor $\left(\mathrm{M}_{2} \mathrm{mAChR}\right)$ transfection was confirmed using a commercially available monoclonal antibody raised against the $\mathrm{M}_{2} \mathrm{mAChR}$ in indirect immunofluorescence assays. Only antibodies raised against an internal region of the receptor peptide were available at the time of assay development. It was therefore necessary to first permeabilise transfected cells prior to antibody application in order for the antibodies to gain access to the epitope. Whilst it would have been preferable for cells to have remained intact, cells permeabilised and stained with the monoclonal anti- $\mathrm{M}_{2} \mathrm{mAChR}$ antibody had clearly circumscribed borders suggesting that the cells were not severely disrupted and the receptor was expressed in the cell membrane (Figure 3.6). This procedure was subsequently used as a positive control in every assay. Negative controls consisted of cells incubated with antibody diluent only, and gave only a low background signal.

\subsubsection{Screening of chagasic sera for anti- $M_{2} m A C h R$ autoantibodies}

None of 57 sera samples from patients with Chagas' disease stained the membranes of $\mathrm{M}_{2}$ mAChR transfected cells. Similarly, Brazilian healthy community control samples failed to produce positive staining (Figure 3.7). 

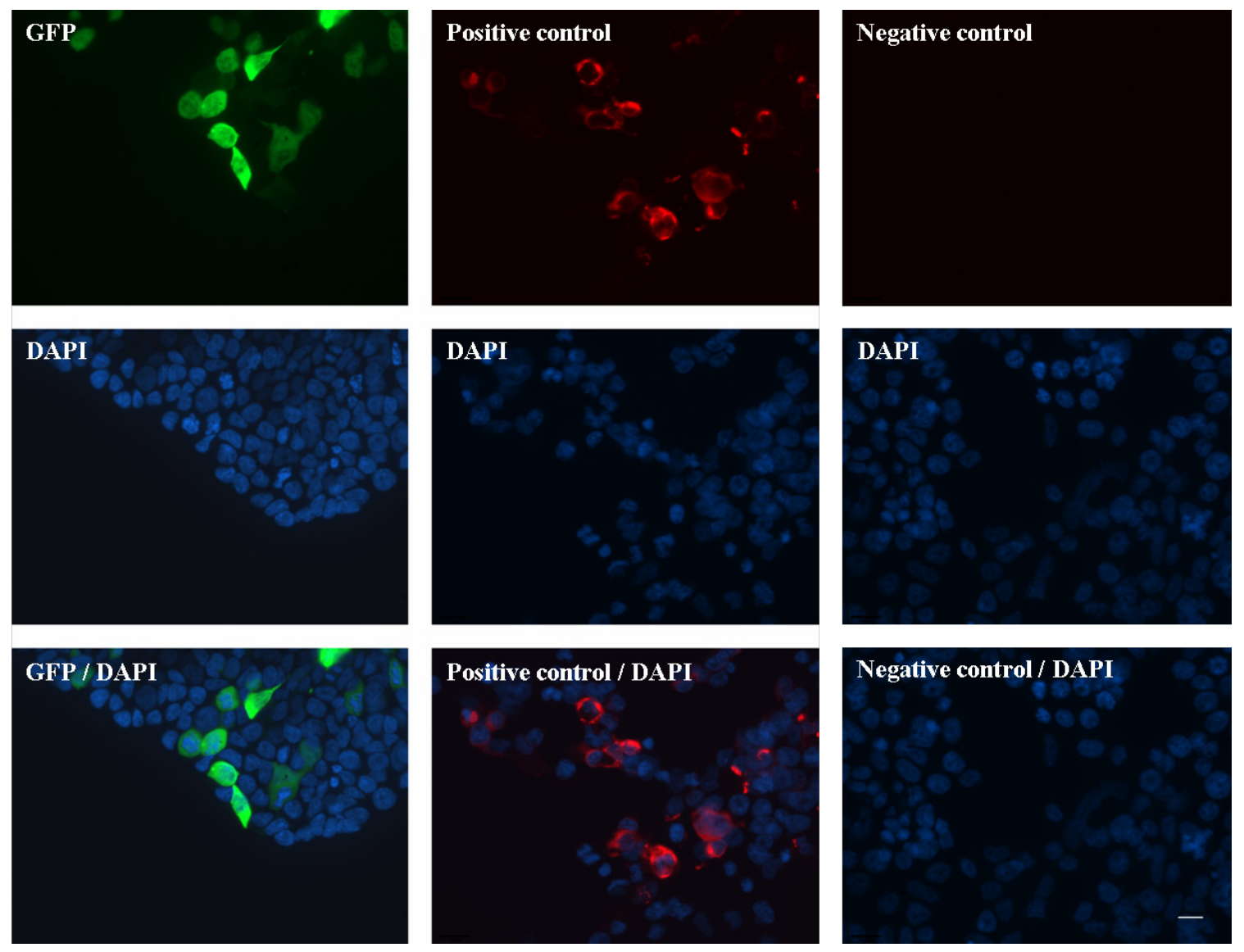

Figure 3.6: Indirect immunofluorescent staining of transfected HEK293 cells with anti-M $M_{2}$ mAChR antibody. GFP-transfected cells auto-fluoresced green demonstrating successful transfection. Cells transfected with $\mathrm{M}_{2} \mathrm{mAChR}$-containing plasmids and incubated with a monoclonal anti$\mathrm{M}_{2}$ antibody served as the positive control and revealed membranous immunostaining. Transfected cells incubated with antibody diluent only served as the negative control and produced no staining. Scale bar $=$ $10 \mu \mathrm{m}$. 

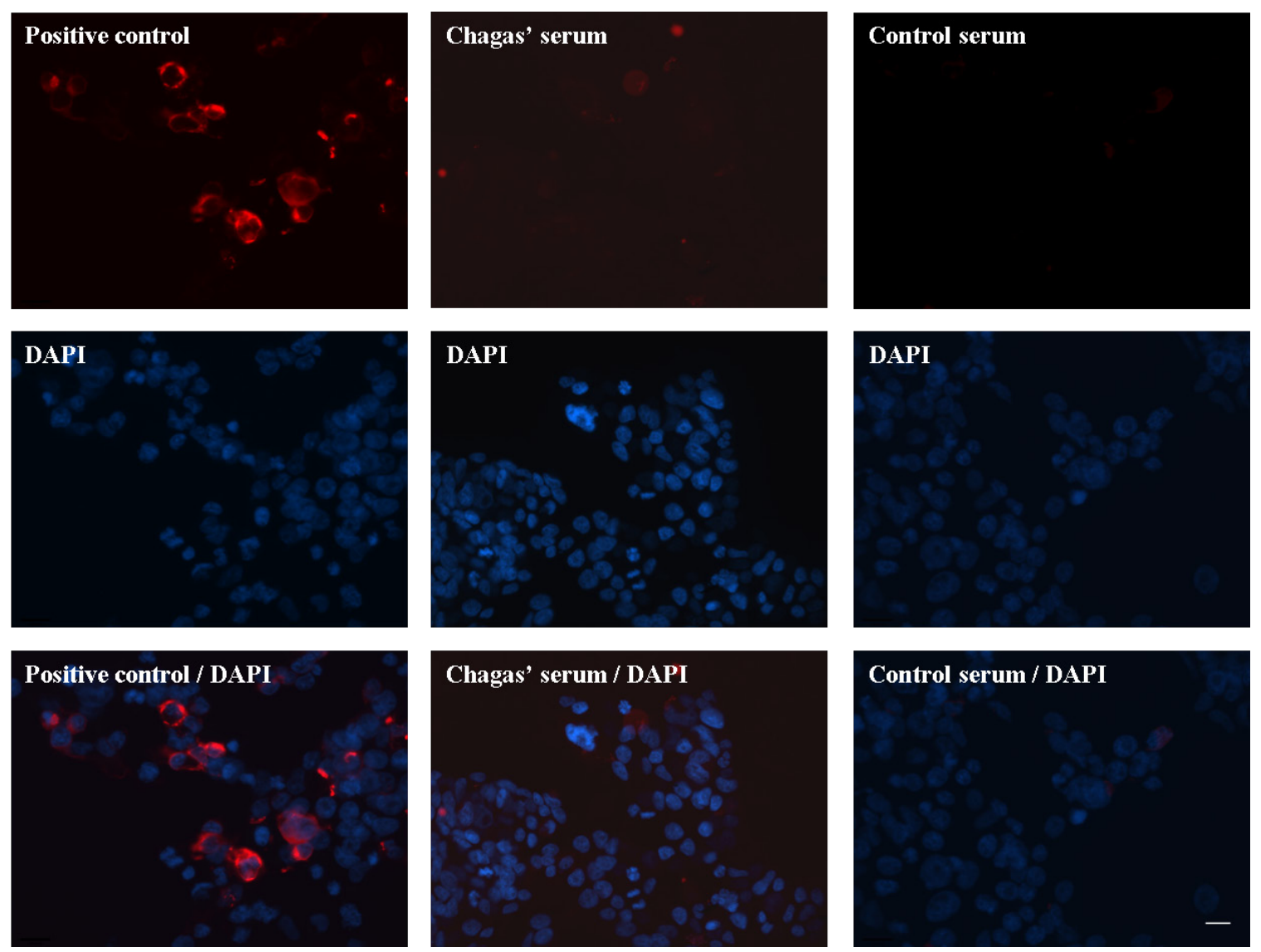

Figure 3.7: Indirect immunofluorescent staining of transfected HEK293 cells with chagasic sera. Chagasic serum samples failed to bind $\mathrm{M}_{2} \mathrm{mAChR}$-transfected cells as demonstrated by indirect immunofluorescence using an anti-human fluorescently-conjugated secondary antibody. Similarly, control serum taken from healthy individuals showed no binding. Scale bar $=10 \mu \mathrm{m}$.

\subsection{FUNCTIONAL IN VITRO EFFECTS OF CHAGASIC SERA}

Following the negativity of the $\mathrm{M}_{2} \mathrm{mAChR}$ cell-based assay we investigated the effects of chagasic sera on human colonic smooth muscle contractions. Due to time constraints the work presented here is preliminary. The effects of sera from three patients with chagasic colonic disease on colonic smooth muscle contractility were investigated. These effects were compared with those of three samples from patients with Chagas' disease and no GI disease and from three healthy community controls. 
Table 3.1: Subjects investigated for sera effects on colonic smooth muscle contractility

\begin{tabular}{ccc}
\hline Subject & Age / sex & Details \\
\hline 1 & $57 \mathrm{~F}$ & Chagasic colonic disease \\
2 & $53 \mathrm{M}$ & Chagasic colonic disease \\
3 & $46 \mathrm{M}$ & Chagasic colonic disease \\
4 & $39 \mathrm{M}$ & Chagas' disease, no sequalae \\
5 & $37 \mathrm{~F}$ & Chagas' disease, no sequalae \\
6 & $35 \mathrm{M}$ & Chagas' disease, no sequalae \\
7 & $40 \mathrm{M}$ & Healthy community control \\
8 & $45 \mathrm{~F}$ & Healthy community control \\
9 & $33 \mathrm{M}$ & Healthy community control \\
\hline
\end{tabular}

\subsubsection{Characterisation of responses of human colonic smooth muscle to electrical field stimulation, atropine and L-NAME}

Responses to electrical field stimulation (EFS) in muscle strips from the same patient were heterogeneous and consisted of contractions (55\%; 21 / 38 strips; $\mathrm{n}=9$ colons), relaxations $(32 \%)$ or no response $(13 \%)$ and were usually followed by a large aftercontraction (Figure 3.8A). Responses could be blocked by the sodium channel blocker tetrodotoxin, $1 \mu \mathrm{M}$ ( $\mathrm{n}=3$ colons), indicating that they were neuronally mediated (Figure 3.8B). The addition of atropine $1 \mu \mathrm{M}$, a mAChR antagonist, prevented contractions during EFS ( $\mathrm{n}=3$ colons) revealing, or facilitating muscle relaxation, and strongly attenuated after-contractions. L-NAME $0.3 \mathrm{mM}$, the nitric oxide synthase inhibitor, prevented muscle relaxation during EFS ( $\mathrm{n}=4$ colons) (Figure 3.8B). 
A
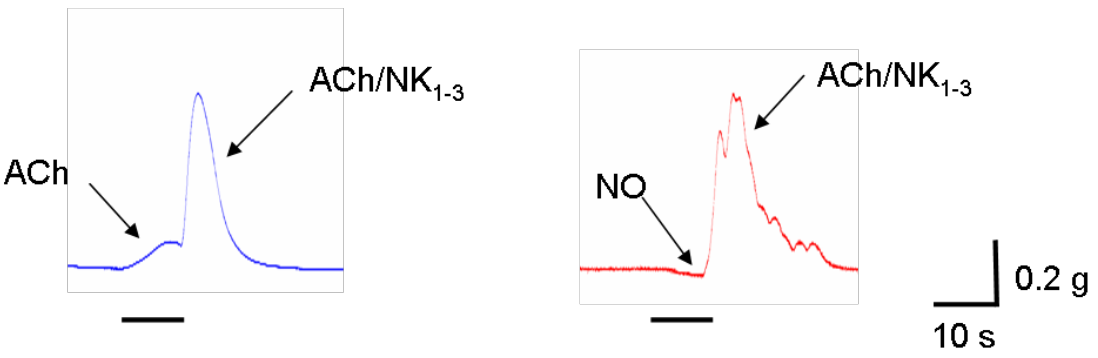

B
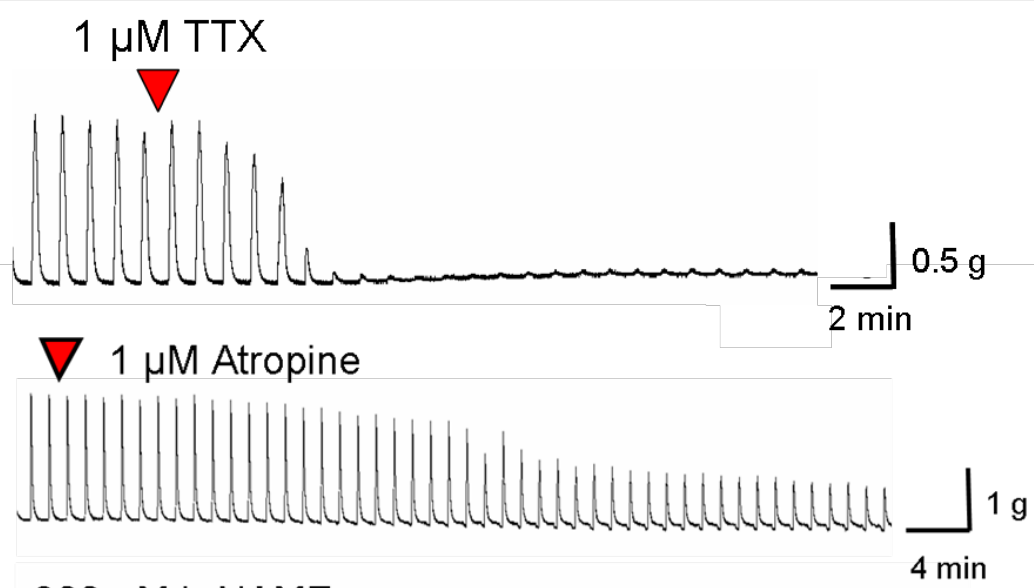
$300 \mu \mathrm{M}$ L-NAME

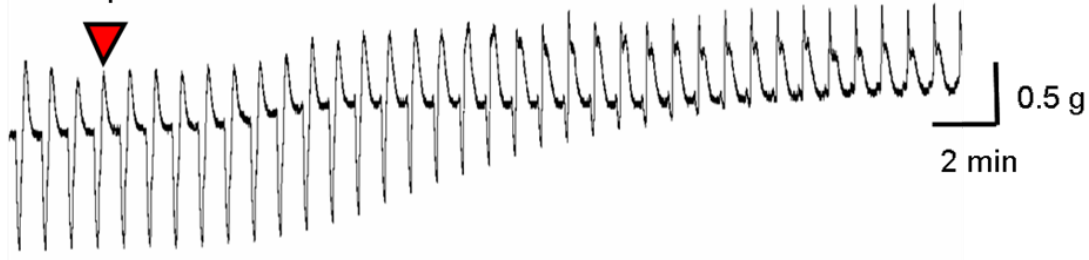

\section{Figure 3.8: Human colonic smooth muscle responses to EFS and pharmacological}

modulation of responses. A: The initial response to EFS was a contraction mediated by acetylcholine, or a relaxation mediated by nitric oxide. Each response was usually followed by a spike representing a large after-contraction. These are mediated by acetylcholine or a neurokinin. (Horizontal bar beneath traces represents the duration of EFS). B: Application of tetrodotoxin prevented EFS responses indicating that they were neuronally mediated. Atropine prevented muscle contraction during EFS, revealing a relaxation, and strongly reduced the magnitude of the after-contraction. L-NAME prevented muscle relaxation during EFS and increased basal muscle tension. $\mathrm{ACh}=$ acetylcholine, $\mathrm{NK}=$ neurokinin. 


\subsubsection{Effect of sera on human colonic smooth muscle responses to EFS}

The only consistent, measurable effect caused by addition of serum to colonic muscle stimulated by EFS was a change in baseline muscle tension. Application of $100 \mu 1$ (bath concentration $1 \%$ ) of serum from three patients with chagasic colonic disease caused a consistent reduction in baseline muscle tension ( $\mathrm{n}=4$ colons / patient; 12/12 relaxations) without a corresponding effect on the magnitude of responses to EFS (Figure 3.9). The overall reduction in muscle tension (mean $\pm \mathrm{SEM}$ ) was $36 \pm 6 \mathrm{mg}(p<0.05$, one sample $\mathrm{t}$ test; Figure 3.12). Contractions during EFS were $104 \pm 13 \%$ of magnitude of contractions before addition of serum; Figure 3.9, which persisted for at least $30 \mathrm{~min}$.

Application of $100 \mu 1$ of serum from three Chagas' disease control patients (with no GI sequalae) produced variable responses in baseline muscle tension. The mean effect on baseline muscle tension was not significant $(2 \pm 15 \mathrm{mg} ; \mathrm{n}=4$ colons / patient; 7/12 relaxations; Figures 3.10 and 3.12). The addition of three serum samples from healthy community controls also produced mixed responses, the mean of which was not significant (-7 $\pm 32 \mathrm{mg} ; 4$ colons / patient; 7/12 relaxations; Figures 3.10 and 3.12). 


\section{Subject 1}

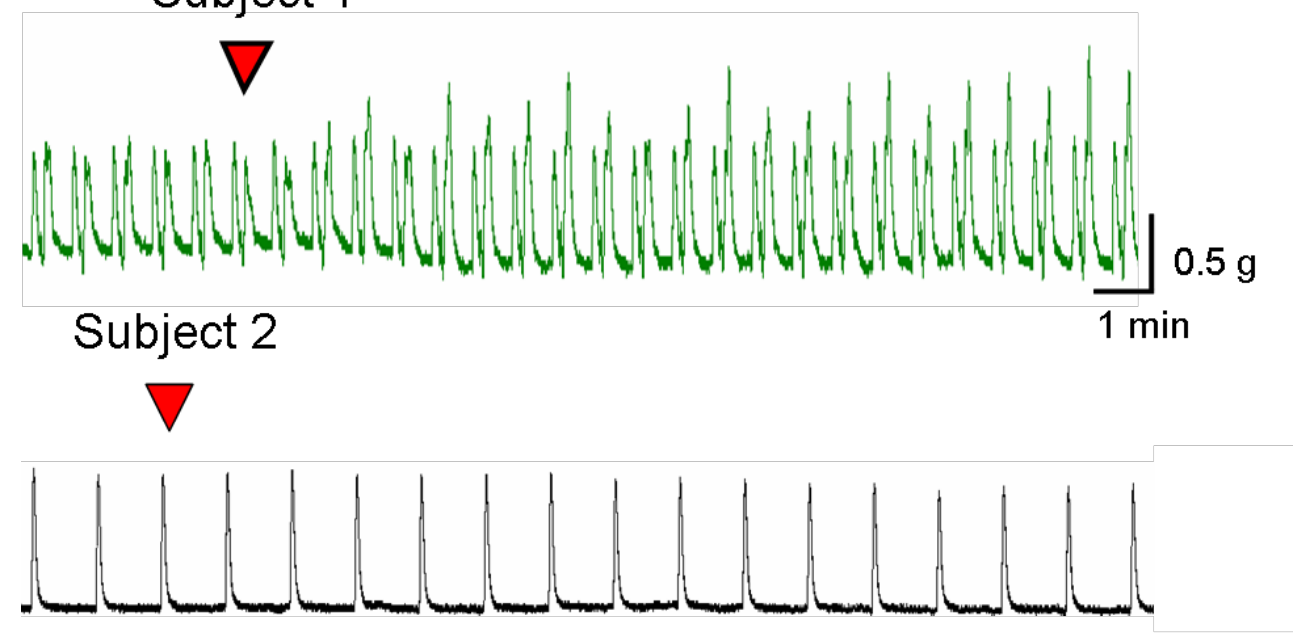

\section{Subject 3}

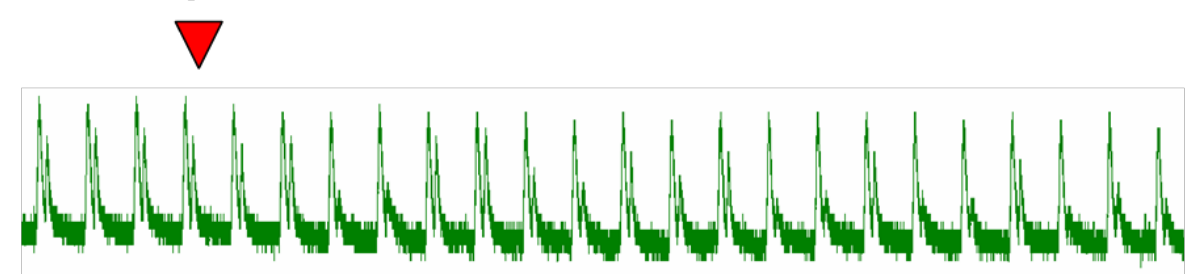

Figure 3.9: Effects of serum from patients with Chagas' colon disease on human colonic smooth muscle responses to EFS. Following addition of serum from patients with Chagas' colon disease, baseline muscle tension always decreased, although to varying degrees. The magnitude of the response during EFS was unchanged. 


\section{Chagas disease control}

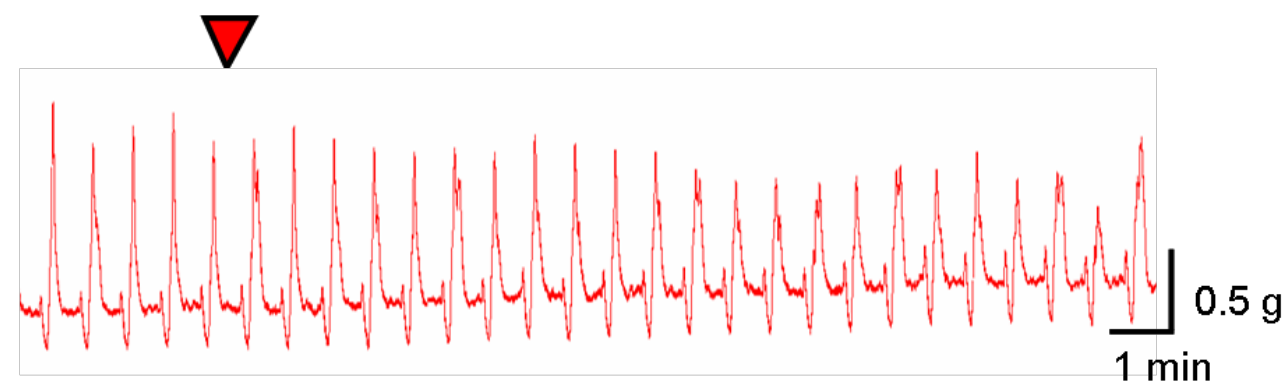

Healthy community control

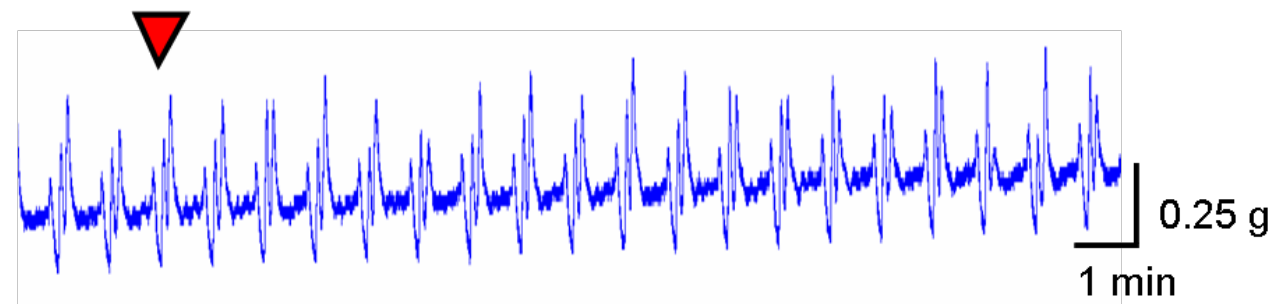

Figure 3.10: Effects of serum from Chagas' disease and healthy controls patients on human colonic smooth muscle responses to EFS. Following addition of serum from patients with Chagas' disease and no GI sequalae or from healthy community controls EFS responses were unchanged. Changes in baseline muscle tension varied, but overall were not significant.

\subsubsection{Effect of chagasic sera on EFS-induced human colonic smooth muscle contractions in the presence of atropine or L-NAME}

To investigate whether the effect of serum from Chagas' colon disease involved a mechanism dependent on $\mathrm{mAChR}$ activation, as reported in previous studies, the effects of these sera were tested in the presence of the mAChR antagonist atropine. Atropine (1 $\mu \mathrm{M})$ was applied to organ baths and allowed $30 \mathrm{~min}$ contact time with muscle strips. Serum was then applied as before. In the presence of atropine, serum continued to cause a reduction in baseline muscle tone. Although the relaxation was slightly attenuated, it was consistent, as before $(-31 \pm 11 \mathrm{mg} ; \mathrm{n}=3$ colons / patient; 9/9 relaxations; Figures 3.11 A 
and 3.12), demonstrating that the observed effect is not dependent on mAChR activation. The response was further examined by application of the nitric oxide synthase inhibitor L-NAME $0.3 \mathrm{mM}$, to investigate whether the effect was dependent on nitric oxide production. Following 30 min compound contact time, addition of serum reduced muscle tension by $54 \pm 11 \mathrm{mg}$ ( $\mathrm{n}=3$ colons / patient; 9/9 relaxations) showing that the response is independent of nitric oxide production (Figures $3.11 \mathrm{~B}$ and 3.12). In further experiments, Tetrodotoxin (TTX), which blocks voltage-gated sodium channels essential for neuronal action potential generation, was applied to muscle to block neuronal activity. Following pre-incubation with $1 \mu \mathrm{M}$ TTX, all three Chagas' colon disease serum samples produced a reduction in basal muscle tension $(-20 \pm 6 \mathrm{mg} ; \mathrm{n}=3$ colons / patient, 7/9 relaxations) which although attenuated compared to toxin free preparations, were not significantly different. This demonstrates that although the effect may perhaps in part involve neuronal mechanisms, it is not dependent on neuronal activity. 
A

\section{Subject 1}

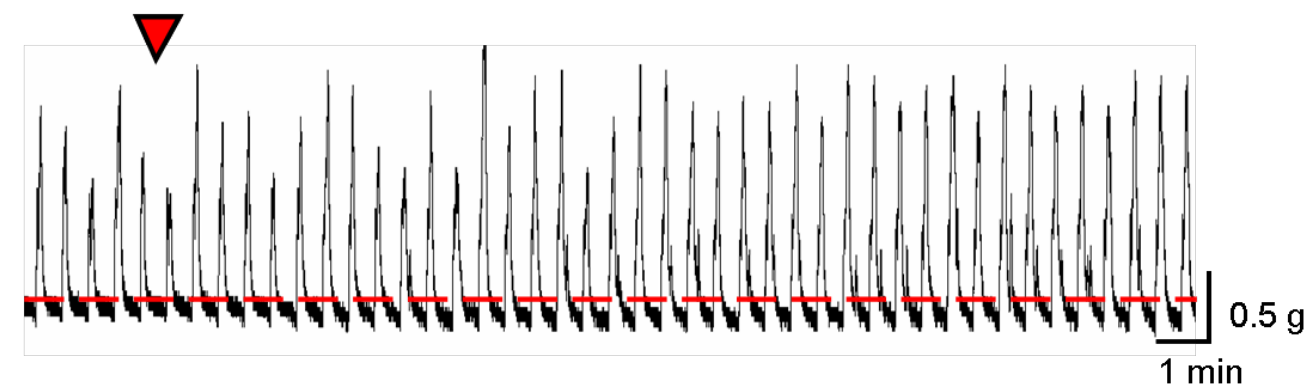

B

Subject 1

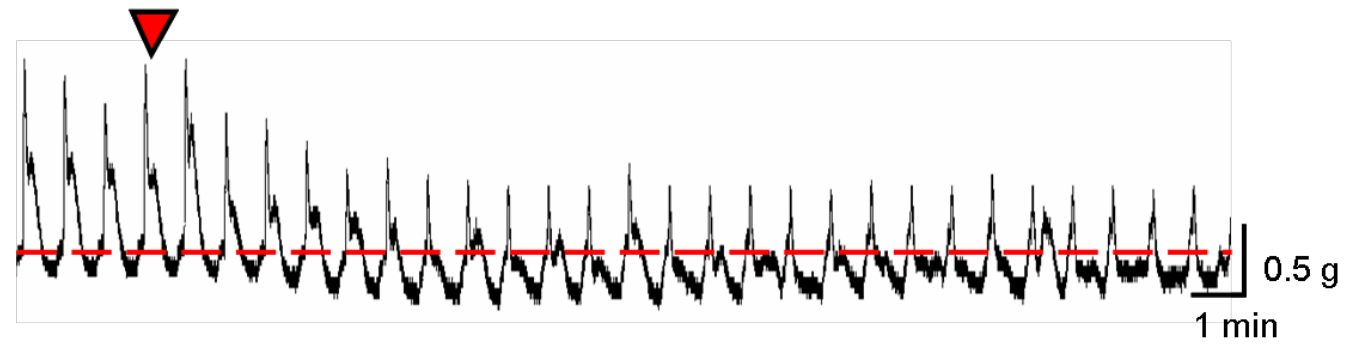

Figure 3.11: Serum effects on human colonic smooth muscle EFS responses in the presence of atropine and L-NAME. The decrease in baseline tension following the addition of serum from a patient with chagasic colonic dysfunction was maintained in the presence of atropine (A) and L-NAME (B) suggesting that circulating factors were not exerting their effects via muscarinic cholinergic receptor activation or via facilitation of nitric oxide synthesis. Red dashed line represents baseline muscle tension prior to addition of serum. 


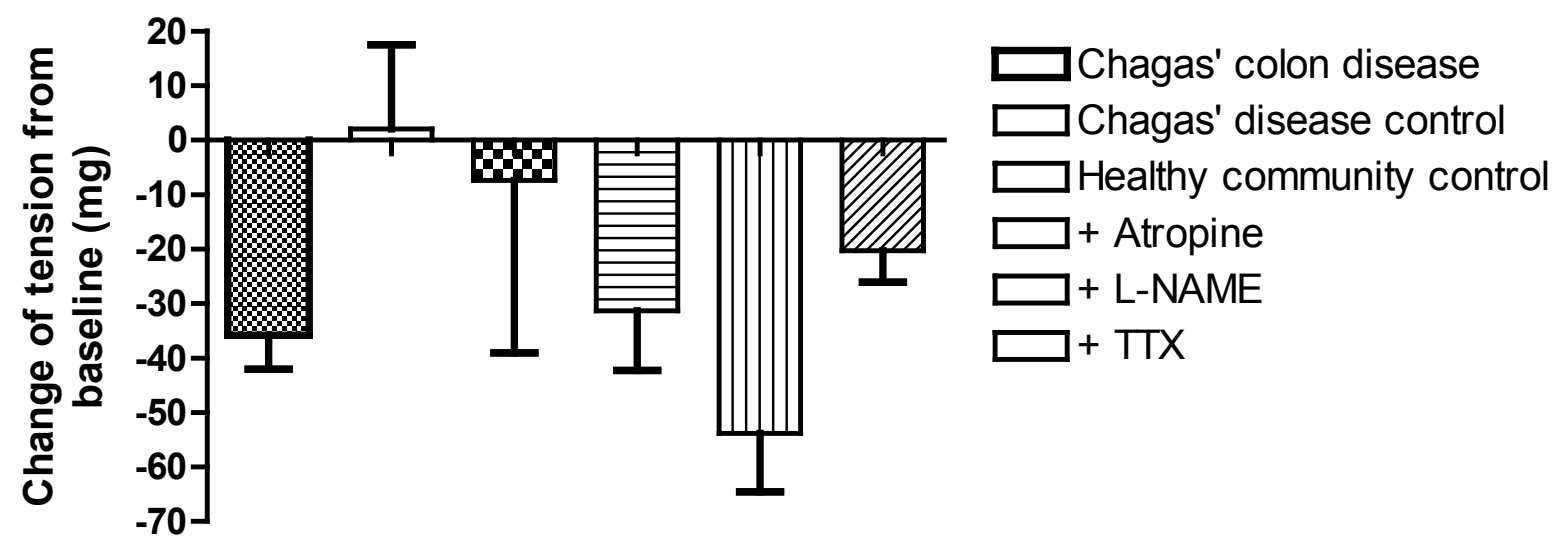

Figure 3.12: Serum effects on human colonic baseline muscle tension in the presence of atropine and L-NAME. Serum from three patients with Chagas' colon disease produced a significant reduction in colonic smooth muscle tone. Serum from three patients with Chagas' disease but no sequalae variably changed basal muscle tone, however these changes were not significant. Responses to serum from three healthy individuals from the same community were more variable, overall producing a reduction in smooth muscle tone. This effect however was not significant. In the presence of 1 $\mu \mathrm{M}$ atropine the relaxant effect on baseline muscle tension was preserved, although slightly attenuated, demonstrating the effect is not dependent on muscarinic cholinergic receptor activation. Relaxations continued in the presence of $300 \mu \mathrm{M}$ L-NAME, and appeared to be slightly facilitated, revealing that they were not the result of nitric oxide production. Tetrodotoxin (TTX; $1 \mu \mathrm{M})$ slightly attenuated but did not abolish the relaxant effect of serum, demonstrating that although this effect may involve neuronal pathways, it is not dependent on neuronal action potentials. 


\subsection{K 1 CHANNEL DISTRIBUTION}

The distribution of six members of the $\mathrm{K}_{V} 1$ VGKC subfamily was investigated immunohistochemically in the human GI tract. Antibody specificity and optimal working dilutions were first determined by immunostaining murine cerebellum.

\subsubsection{MURINE CEREBELLUM}

The distribution of $\mathrm{K}_{\mathrm{V}} 1$ channels has been previously established in rodent cerebellum and allowed us to confirm the specificity of our antibodies and determine optimal working dilutions. No staining was seen in the negative control sections where the primary anti- $\mathrm{K}_{\mathrm{V}} 1$ antibodies were omitted but the secondary detection system was used as normal, indicating areas of staining seen in test sections were not due to non-specific secondary antibody binding (Figure 3.13). The staining patterns we observed in mouse cerebellum with the anti-K $\mathrm{K}_{\mathrm{V}} 1$ antibodies (Figure 3.14) were the same as those described in previous reports (Veh et al. 1995; Chung et al. 2001). $\mathrm{K}_{\mathrm{V}} 1.1$ was found in basket cell axon terminals around Purkinje cells and also weakly in the neuropil within the granule cell layer. $\mathrm{K}_{\mathrm{V}} 1.2$ immunoreactivity also appeared in basket cell axon terminals, in greater abundance to $K_{V} 1.1$. Immunoreactivity for $K_{V} 1.3$ was found in Purkinje cells, Golgi-type II cells of the granular layer and basket cells of the molecular layer. Purkinje cell bodies were stained with anti- $\mathrm{K}_{V} 1.4,1.5$ and 1.6. $\mathrm{K}_{\mathrm{V}} 1.6$ immunoreactivity was also found in basket cells of the molecular layer and Golgi type II cells of the granular layer. No staining was seen in the negative control sections. Human and mouse $\mathrm{K}_{V} 1$ channels are homologous. Therefore, having confirmed that the anti-K $\mathrm{K}_{\mathrm{V}} 1$ antibodies bound as expected in murine cerebellum, we proceeded to immunohistochemically investigate $K_{V} 1$ distribution in the human GI tract. For the purposes of this thesis and considering the questionable value of quantitative immunohistochemistry (Polak et al. 2003), this is a descriptive study only. 


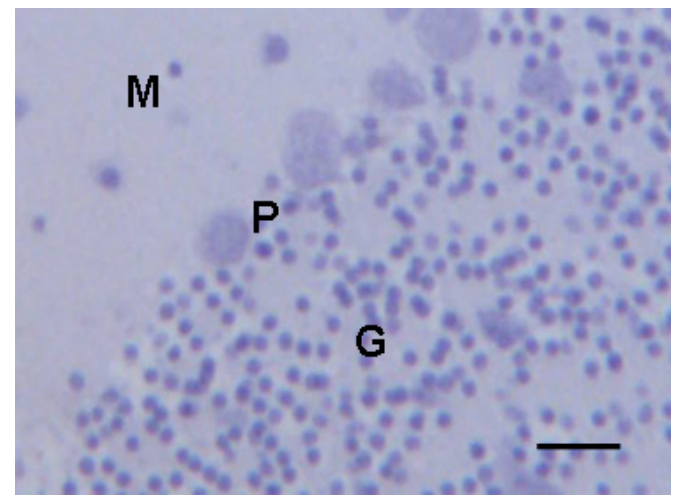

Figure 3.13: Negative immunostaining control of mouse cerebellum. Sections of mouse cerebellum incubated with antibody diluent in the absence of anti-KV1 antibodies showed no staining confirming that the secondary detection system, which was used as normal, was not responsible for any staining subsequently observed. $\mathrm{G}=$ granule cell layer, $\mathrm{M}=$ molecular layer, $\mathrm{P}=$ Purkinje cell. Scale $\mathrm{bar}=$ $25 \mu \mathrm{m}$. 

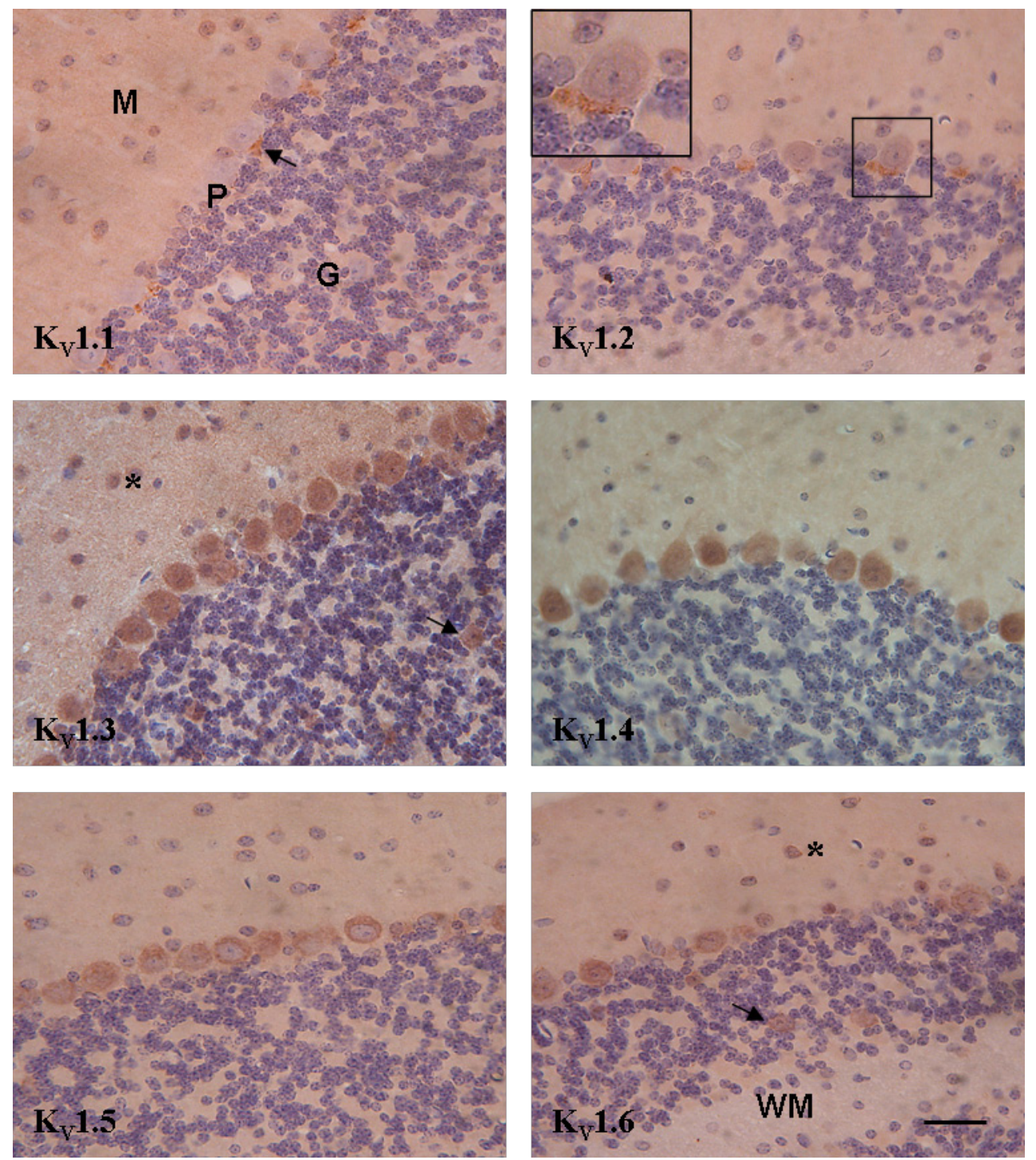

Figure 3.14: Anti- $K_{V} 1$ immunostaining of mouse cerebellum. $K_{V} 1.1$ and 1.2 localised specifically to basket cell axon terminals around Purkinje cells (arrow and inset, respectively). $\mathrm{K}_{\mathrm{V}} 1.3$ was found in Purkinje cell bodies, Golgi type II cells of the granular layer (arrow) and basket cells of the molecular layer (star). $\mathrm{K}_{\mathrm{V}} 1.4$ immunoreactivity was also found in Purkinje cell bodies. $\mathrm{K}_{\mathrm{V}} 1.5$ strongly stained Purkinje cell bodies. $\mathrm{K}_{\mathrm{V}} 1.6$ localised to Purkinje cell bodies, basket cells (star) and Golgi type II cells of the granular layer (arrow). $\mathrm{M}=$ molecular layer, $\mathrm{P}=$ Purkinje cell layer, $\mathrm{G}=$ granular layer; $\mathrm{WM}$, white matter. Scale bar $=25 \mu \mathrm{m}$. 


\subsubsection{OESOPHAGUS}

Oesophageal epithelium

The oesophageal epithelium did not show immunopositivity for any of the six $K_{V} 1$ channel subunits (Figure 3.15).

\section{Oesophageal neurons}

Anti- $K_{V} 1.1$ strongly immunostained cell bodies of the myenteric plexus. Cell bodies also exhibited moderate cytoplasmic immunoreactivity for $K_{V} 1.5$. Anti- $K_{V} 1.2$ and $K_{V} 1.4$ weakly stained myenteric cell bodies, $\mathrm{K}_{\mathrm{V}} 1.2$ exhibited a cytoplasmic distribution and $\mathrm{K}_{\mathrm{V}} 1.4$ staining cell nuclei. Anti- $\mathrm{K}_{\mathrm{V}} 1.3$ and $\mathrm{K}_{\mathrm{V}} 1.6$ immunostaining was not above background levels (Figure 3.16).

\section{Oesophageal smooth muscle}

$\mathrm{K}_{\mathrm{V}} 1.5$ was found to be localised myocytes of the oesophageal muscularis propria and arterioles. Anti- $\mathrm{K}_{\mathrm{V}} 1.5$ immunostaining identified discrete patches of punctate staining, which often appeared to be intracellular, specifically peri-nuclear, in distribution (Figure 3.17). No other channel subunit was identified in smooth muscle cells. 

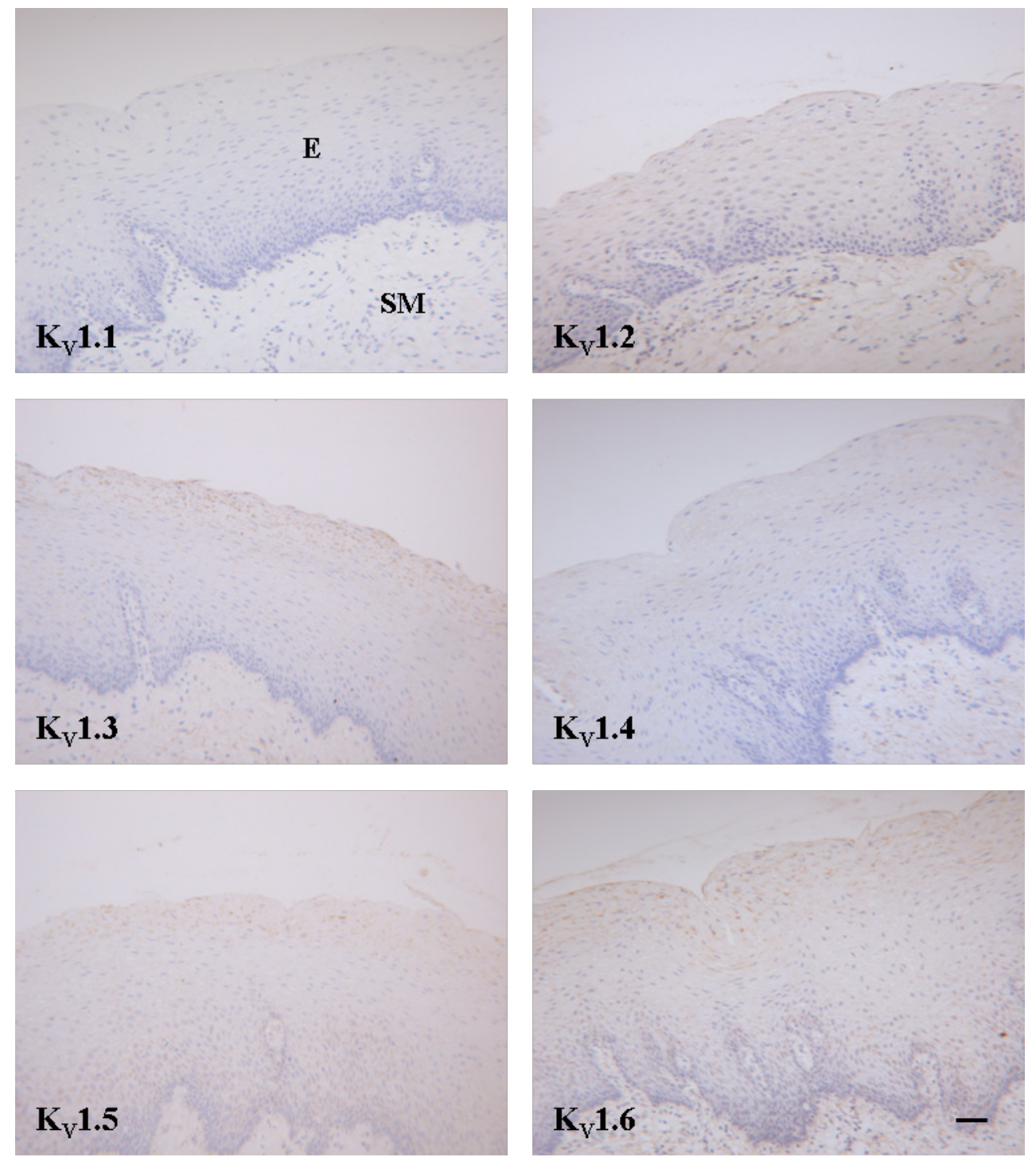

Figure 3.15: Anti-KV 1 immunostaining of oesophageal epithelium. All cells of the squamous epithelium were negative for $\mathrm{K}_{\mathrm{V}}$ 1.1-1.6 immunoreactivity. E, epithelium; SM, submucosa. Scale bar $=50 \mu \mathrm{m}$. 

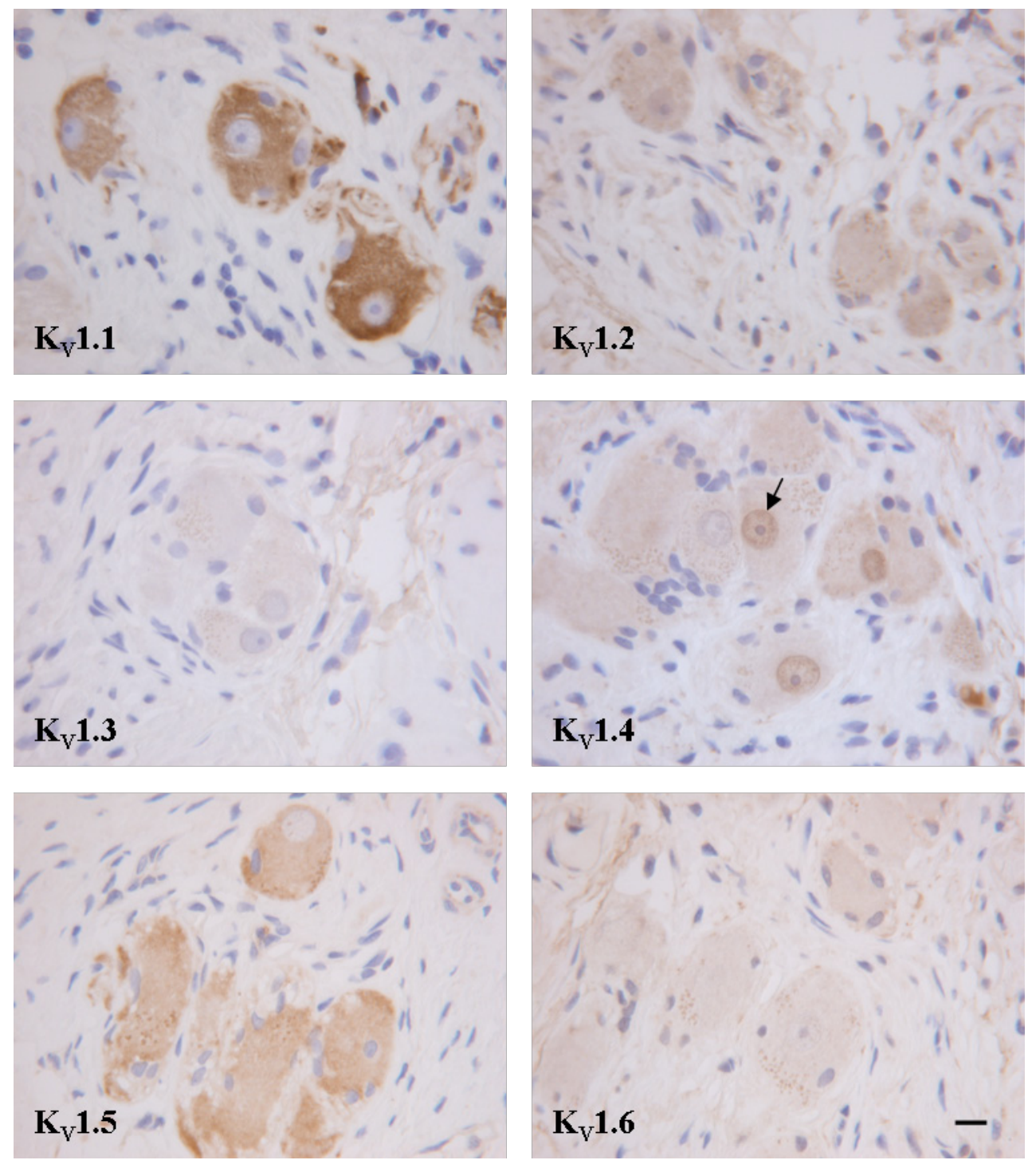

Figure 3.16: Anti-KV1 immunostaining of human oesophageal myenteric ganglia.

$\mathrm{K}_{\mathrm{V}} 1.1$ was found in neuronal cell bodies in a cytoplasmic distribution. $\mathrm{K}_{\mathrm{V}} 1.2$ immunostaining was weak in neuronal cell bodies. $\mathrm{K}_{\mathrm{V}} 1.3$ was not detected in oesophageal myenteric ganglia. Neuronal nuclei were weakly immunopositive for $\mathrm{K}_{\mathrm{V}} 1.4$ (arrow). $\mathrm{K}_{\mathrm{V}} 1.5$ was distributed in the cytoplasm of cell bodies and $\mathrm{K}_{\mathrm{V}} 1.6$ was not found to be present. Scale bar $=10 \mu \mathrm{m}$. 

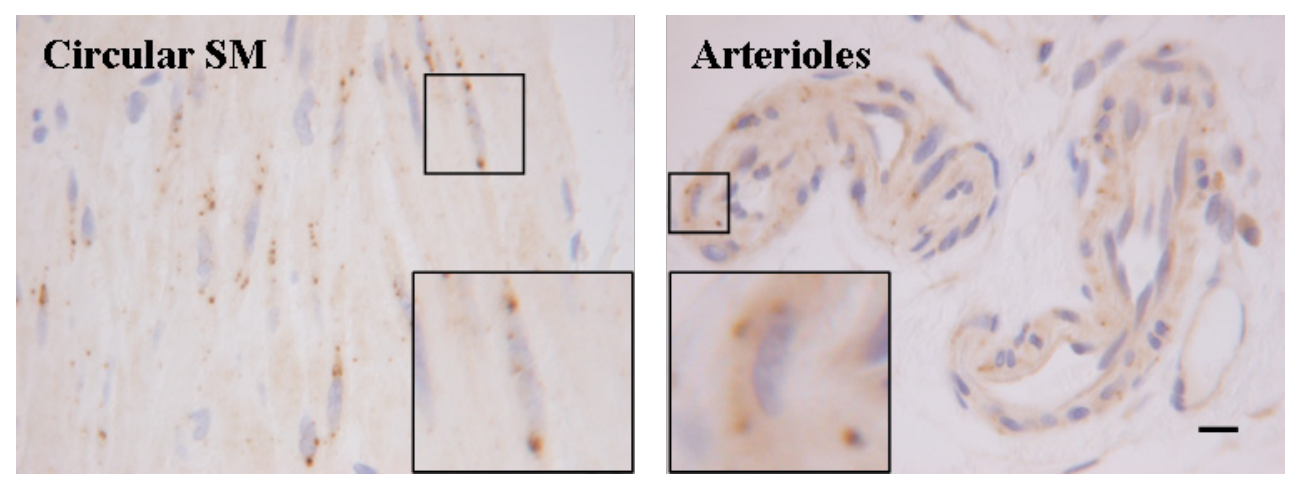

Figure 3.17: Anti-K 1.5 immunostaining of human oesophageal smooth muscle.

$\mathrm{K}_{\mathrm{V}} 1.5$ containing puncta were abundant throughout muscularis propria and in arteriolar myocytes. These puncta were often observed to be intracellular, and often peri-nuclear (inset). Scale bar $=10 \mu \mathrm{m}$.

\subsubsection{STOMACH}

\section{Gastric mucosa}

$\mathrm{K}_{\mathrm{V}} 1$ immunostaining of the gastric epithelium labelled Chief cells (as identified by H\&E appearance) where intracellular granular staining was present. Immunostaining for $\mathrm{K}_{\mathrm{V}} 1.1$, $\mathrm{K}_{\mathrm{V}} 1.2, \mathrm{~K}_{\mathrm{V}} 1.3$ and $\mathrm{K}_{\mathrm{V}} 1.4$ was strong compared with $\mathrm{K}_{\mathrm{V}} 1.5$ and $\mathrm{K}_{\mathrm{V}} 1.6$ which were moderate. Parietal cells and surface epithelial cell staining was not above background levels (Figure 3.18). The muscularis mucosa was also negative (data not shown).

\section{Gastric neurons}

$\mathrm{K}_{\mathrm{V}} 1$ subunits exhibited a selective distribution in gastric submucosal and myenteric ganglia (Figures 3.19 and 3.20, respectively). Cell bodies and the neuropil of submucosal and myenteric ganglia were weakly immunopositive for $\mathrm{K}_{\mathrm{V}} 1.1$. Cell bodies of both plexi intensely stained for $\mathrm{K}_{\mathrm{V}} 1.2$. Anti- $\mathrm{K}_{\mathrm{V}} 1.3$ immunostaining also localised to cell bodies with the additional observation of nuclear immunostaining. Anti- $\mathrm{K}_{\mathrm{V}} 1.4$ failed to stain submucosal cells bodies, however immunostaining of $\mathrm{K}_{\mathrm{V}} 1.4$ was scattered around the periphery of myenteric ganglion in a punctate distribution. At light microscopic 
magnifications it was not possible to identify these channel-rich structures. Certain myenteric cell bodies were also strongly immunopositive for $\mathrm{K}_{\mathrm{V}} 1.4$. $\mathrm{K}_{\mathrm{V}} 1.5$ immunostaining was moderate to strong in submucosal and myenteric cell bodies with. $\mathrm{K}_{\mathrm{V}} 1.6$ immunostaining was not consistently above background levels. Antibodies to $\mathrm{K}_{\mathrm{V}} 1$ subunits did not label circular or longitudinal layers of muscularis externae. 

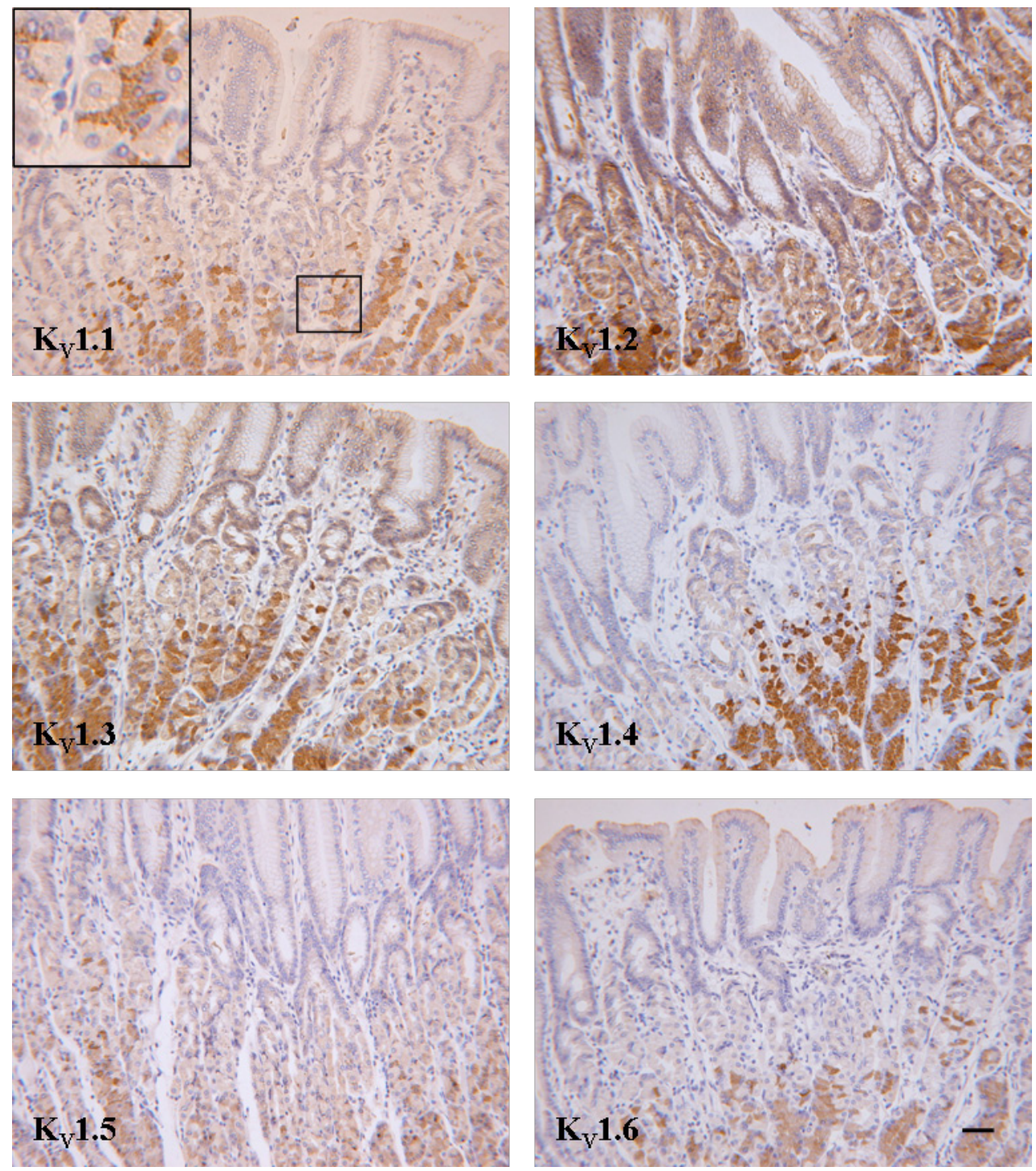

Figure 3.18: $K_{V} 1$ immunostaining of human gastric epithelium. Cells located towards the base of gastric glands identified as Chief cells were strongly stained with anti-K $\mathrm{K}_{\mathrm{V}} 1.1$ (inset; dark brown), $\mathrm{K}_{\mathrm{V}} 1.2, \mathrm{~K}_{\mathrm{V}} 1.3$ and $\mathrm{K}_{\mathrm{V}} 1.4$ antibodies. Chief cells were moderately stained with antibodies against $\mathrm{K}_{\mathrm{V}} 1.5$ and $\mathrm{K}_{\mathrm{V}}$ 1.6. Surface epithelial cells and parietal cells were negative (membranous staining of surface epithelial cells seen in $\mathrm{K}_{\mathrm{V}} 1.6$ slide was considered to be artefact). Scale bar $=50 \mu \mathrm{m}$. 

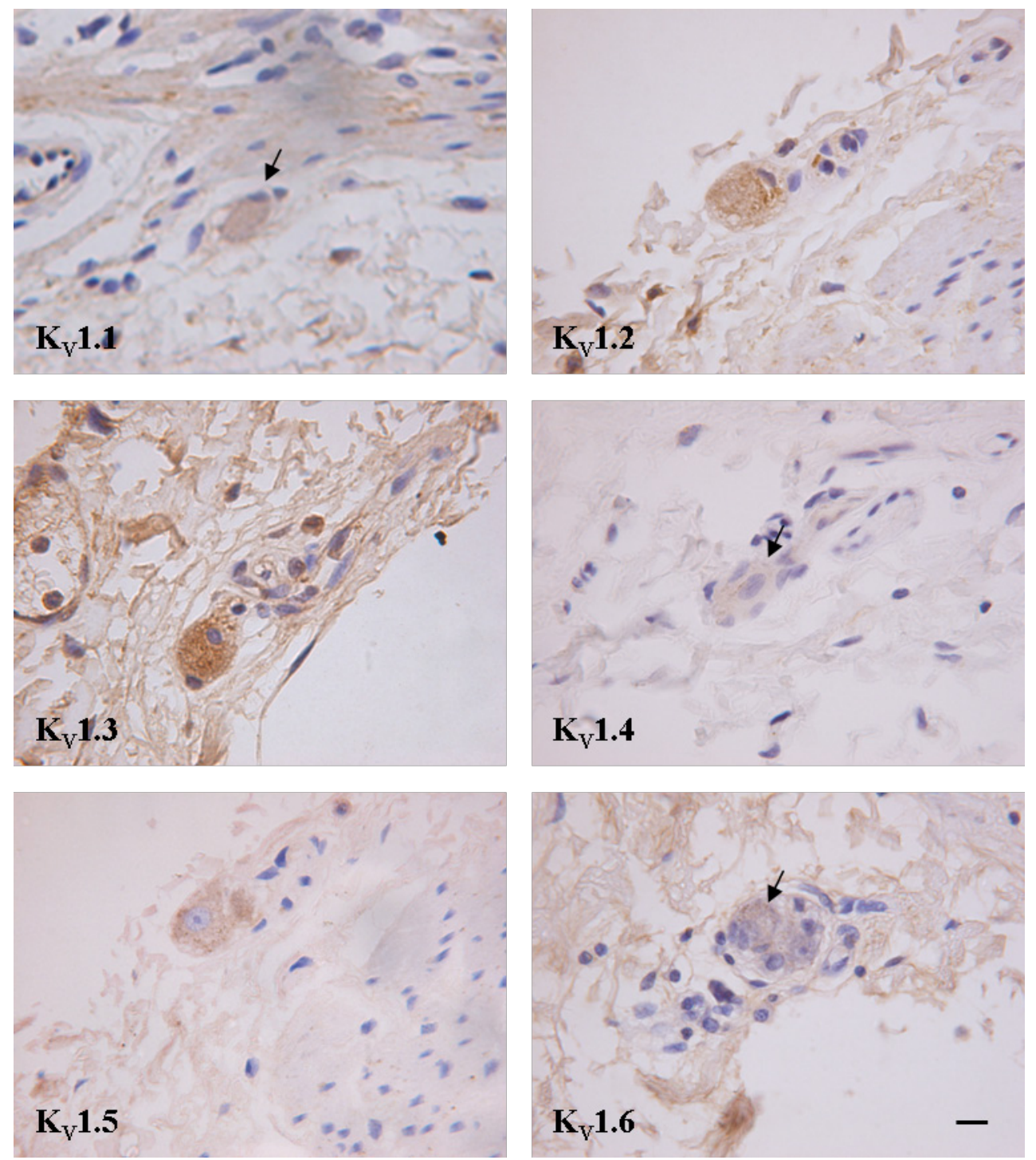

Figure 3.19: $K_{V} 1$ immunostaining of human gastric submucosal neurons. Anti- $K_{V} 1.1$

weakly stained neuronal cell bodies (arrow) whereas $\mathrm{K}_{\mathrm{V}} 1.2$ and 1.3 were strong. $\mathrm{K}_{\mathrm{V}} 1.4$ and 1.6 were not found to be expressed by gastric submucosal ganglia (arrow). Anti- $\mathrm{K}_{\mathrm{V}} 1.5$ moderately stained cell bodies. Scale bar $=10 \mu \mathrm{m}$. 

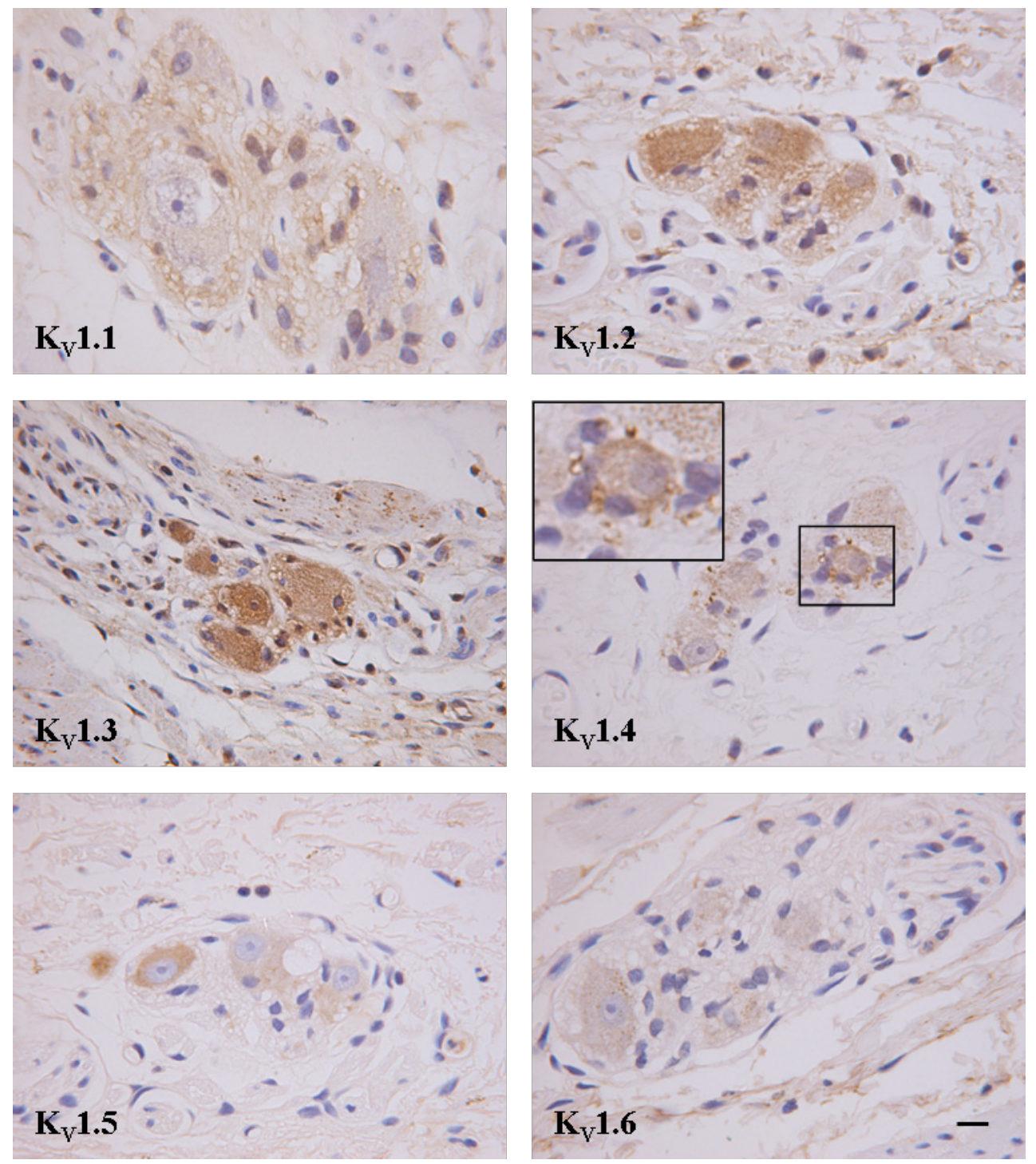

Figure 3.20: $K_{V} 1$ immunostaining of human gastric myenteric ganglia. Gastric myenteric neurons were immunopositive for $\mathrm{K}_{\mathrm{V}} 1.1-1.5$. Anti- $\mathrm{K}_{\mathrm{V}} 1.2,1.3$ and 1.5 antibodies selectively labelled neuronal cell bodies. $\mathrm{K}_{\mathrm{V}} 1.1$ was also present in the surrounding neuropil. $\mathrm{K}_{\mathrm{V}} 1.4$ exhibited a unique punctuate distribution at the periphery of ganglia and certain neuronal cell bodies (inset). $\mathrm{K}_{\mathrm{V}} 1.6$ immunostaining was not above background. Scale bar $=10 \mu \mathrm{m}$. 


\subsubsection{JEJUNUM}

\section{Jejunal mucosa}

As in the stomach, $K_{V} 1$ immunostaining was localised to jejunal epithelial cells (Figure 3.21). Both absorptive enterocytes and goblet cells were immunopositive for both cytoplasm and surface membranes, albeit moderately. Epithelial cells towards the villi tips were more strongly stained than those of the crypts. $\mathrm{K}_{\mathrm{V}} 1.2$ was the most abundant subunit in jejunal epithelium. $\mathrm{K}_{\mathrm{V}} 1.1$ and $\mathrm{K}_{\mathrm{V}} 1.3$ immunostains were moderately positive whereas anti-K $\mathrm{K}_{\mathrm{V}} 1.4$ and $\mathrm{K}_{\mathrm{V}} 1.5$ were weak. $\mathrm{K}_{\mathrm{V}} 1.6$ failed to stain above background levels. Anti- $K_{V} 1.1,1.3,1.4$ and 1.6 antibodies specifically immunolabelled a subset of cells, presumed to be enteroendocrine cells (based on their morphology) of jejunal epithelium, particularly in crypts (Figure 3.21, arrows). The muscularis mucosa was negative for all six channel subunits.

\section{Jejunal neurons}

$\mathrm{K}_{\mathrm{V}} 1.1$ immunostaining was strong in selected ganglion cell bodies of the submucosal plexus and stained the neuropil of myenteric ganglia. $\mathrm{K}_{\mathrm{V}} 1.2$ localised specifically to certain cell bodies of both plexuses, with little staining of the neuropil. Submucosal and myenteric cells, including cell nuclei, were strongly positive for $K_{V} 1.3$. No specific pattern regarding positivity of large vs. small neurons was discernable. As in the stomach, immunoreactivity for $\mathrm{K}_{\mathrm{V}} 1.4$ was scattered throughout enteric ganglia in a peripheral punctate distribution with very little staining of cell bodies. $K_{V} 1.5$ appeared very specific for cell bodies of both ganglia with no staining of the neuropil. Submucosal and myenteric neuronal cell bodies were negative for $\mathrm{K}_{V} 1.6$ (Figure 3.22 and 3.23). Smooth muscle cells of the jejunum appeared negative for all $\mathrm{K}_{\mathrm{V}} 1$ immunostaining (not shown). 

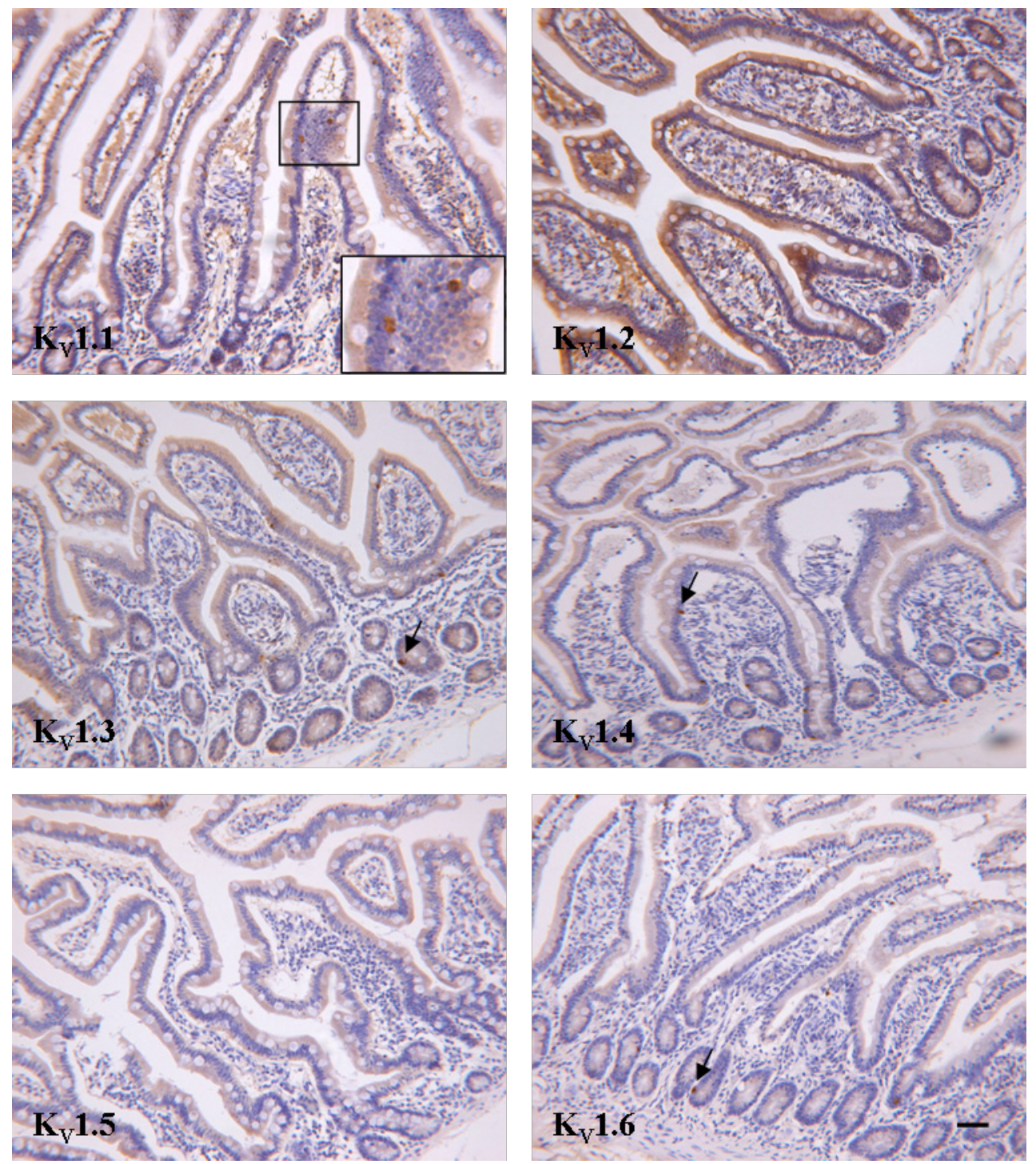

Figure 3.21: $K_{V} 1$ immunostaining of human jejunal mucosa. $K_{V} 1$ subunits were specifically observed in surface epithelial cells, $\mathrm{K}_{\mathrm{V}} 1.2$ appearing to be most abundant. Crypt epithelial cells were also stained, although at a lower intensity. $\mathrm{K}_{\mathrm{V}} 1.1$ and 1.3 immunostaining were moderate, $\mathrm{K}_{\mathrm{V}} 1.4$ and $\mathrm{K}_{\mathrm{V}} 1.5$ were weak and $\mathrm{K}_{\mathrm{V}} 1.6$ was not above background staining. A subset of cells, presumed by appearance to be enteroendocrine, were strongly stained at their basal aspect by anti- $\mathrm{K}_{\mathrm{V}} 1.1$ (inset), 1.3, 1.4 and 1.6 (arrows). Scale bar $=50 \mu \mathrm{m}$. 

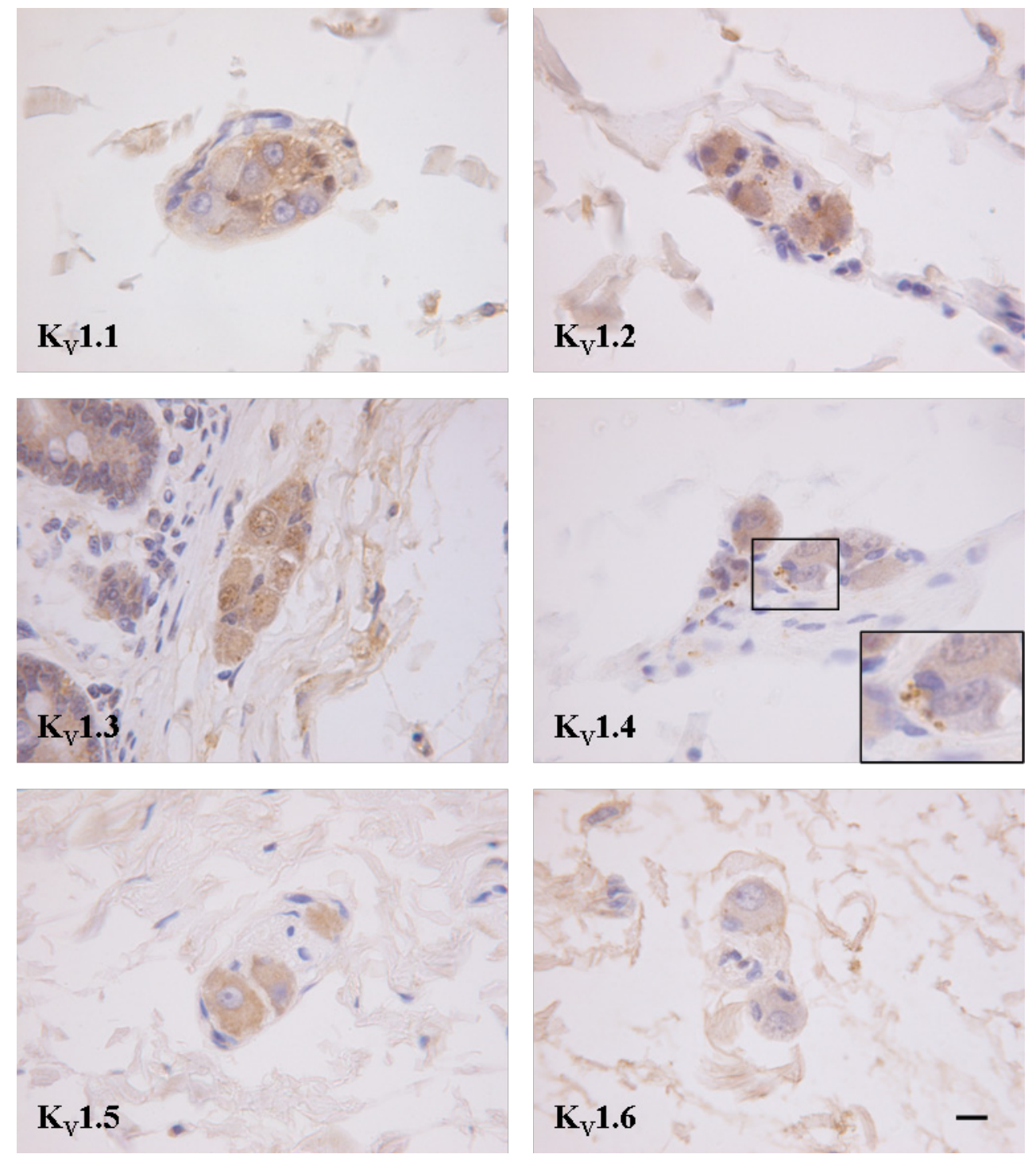

Figure 3.22: $K_{V} 1$ immunostaining of human jejunal submucosal ganglia. Jejunal submucosal neurons were moderate-strongly stained with anti- $\mathrm{K}_{\mathrm{V}} 1$ antibodies. $\mathrm{K}_{\mathrm{V}} 1.1,1.2,1.3$ and 1.5 were localised to a subset of neuronal cells bodies. In addition to cytoplasmic staining, $\mathrm{K}_{\mathrm{V}} 1.3$ also exhibited nuclear staining. $\mathrm{K}_{\mathrm{V}} 1.4$ uniquely immunostained discrete structures around ganglion cells bodies and ganglia periphery, in a punctate distribution (inset). $\mathrm{K}_{\mathrm{V}} 1.6$ failed to stain above background. Scale bar $=10$ $\mu \mathrm{m}$. 

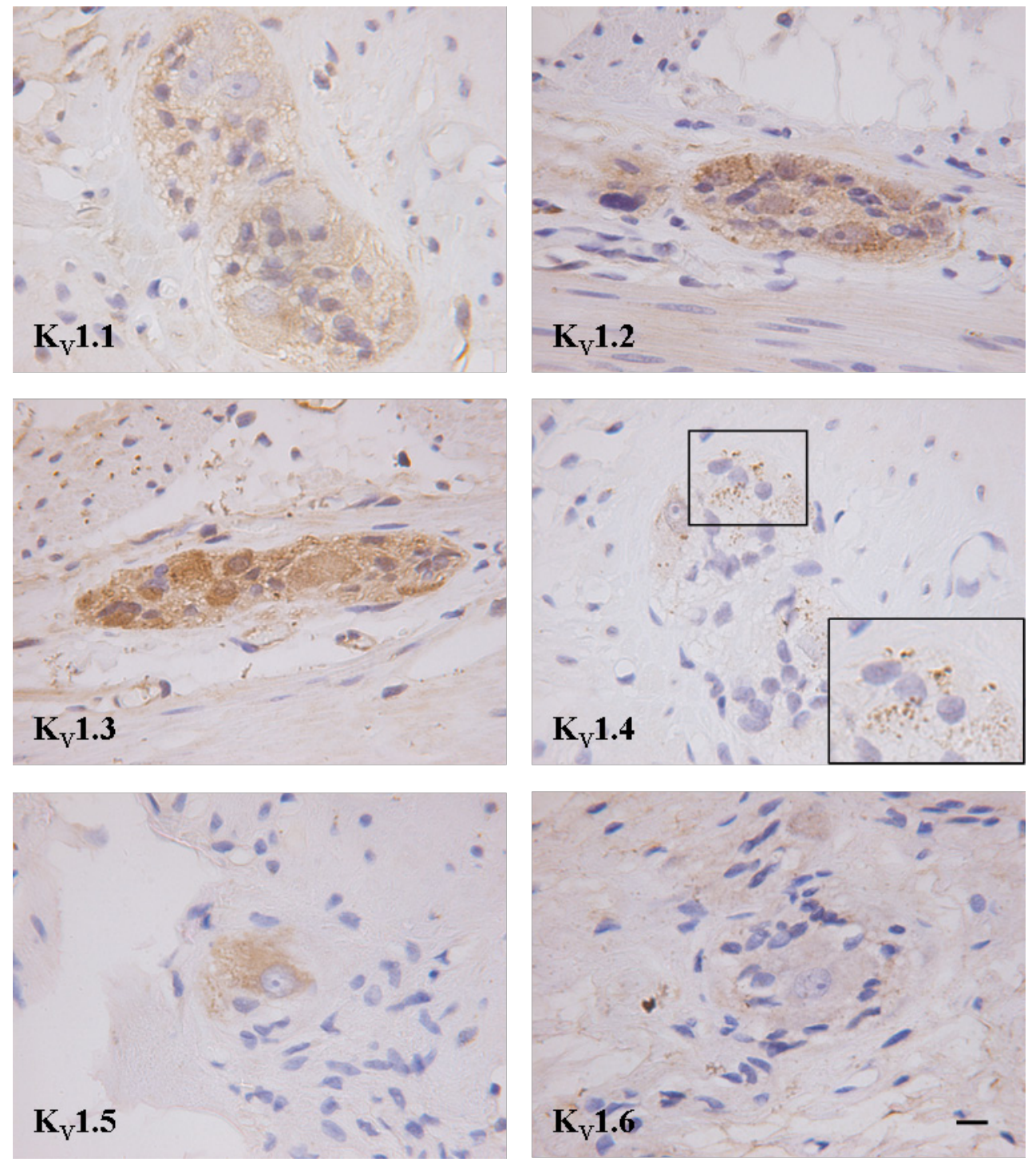

Figure 3.23: $K_{V} 1$ immunostaining of jejunal myenteric ganglia. Anti- $K_{V} 1.1$ immunostained the neuropil of myenteric ganglia, $\mathrm{K}_{\mathrm{V}} 1.2$ and 1.5 localised to neuronal cell bodies and $\mathrm{K}_{\mathrm{V}} 1.3$ immunostained both, including neuronal nuclei. $\mathrm{K}_{\mathrm{V}} 1.4$ stained discrete fine punctate patches scattered around the periphery of ganglia and certain cell bodies (inset). $K_{V} 1.6$ failed to stain ganglia. Scale bar $=10 \mu \mathrm{m}$. 


\subsubsection{ILEUM}

\section{Ileal mucosa}

The transition between immunopositive and immunonegative cells from villi to crypts was more pronounced in the ileum (Figure 3.24). Ileal enterocytes and goblet cells were strongly immunopositive for $\mathrm{K}_{\mathrm{V}} 1.2$ and $\mathrm{K}_{\mathrm{V}} 1.3 . \mathrm{K}_{\mathrm{V}} 1.1$ and $\mathrm{K}_{\mathrm{V}} 1.5$ also localised to surface epithelial cells although at a lower intensity. As in the jejunum, anti- $K_{V} 1.4$ and $K_{V} 1.6$ antibodies only weakly stained epithelial cells. Presumed enteroendocrine cells expressed a high density of $\mathrm{K}_{\mathrm{V}} 1.1,1.2,1.3,1.4$ and 1.6 in their basal regions. Cells of the lamina propria and muscularis mucosa were negative.

\section{Ileal neurons}

Immunostaining for $\mathrm{K}_{\mathrm{V}} 1.1$ was largely confined to the neuropil of myenteric and submucosal ganglia. $\mathrm{K}_{\mathrm{V}} 1.2$ was selectively present in high density in ganglion cell bodies. $\mathrm{K}_{\mathrm{V}} 1.3$ exhibited nuclear localisation, appearing highly expressed in submucosal ganglion cell bodies and moderately in myenteric neurons. As in the stomach and jejunum, $\mathrm{K}_{V} 1.4$ immunostaining was scattered throughout ganglia of both plexi in a punctate distribution, which appears unique to this subunit. Certain ileal ganglion cell bodies also appeared weakly $K_{V} 1.4$ positive. A high density of $K_{V} 1.5$ subunits was observed in submucosal ganglion cell bodies and a moderate concentration in myenteric ganglion cells. $\mathrm{K}_{\mathrm{V}} 1.6$ immunostaining was only weakly positive in submucosal cell bodies and was not present in myenteric cell bodies (Figure 3.25 and 3.26). As in the stomach and jejunum, ileal smooth muscle cells were not immunostained with anti- $\mathrm{K}_{\mathrm{V}} 1$ antibodies (not shown). 

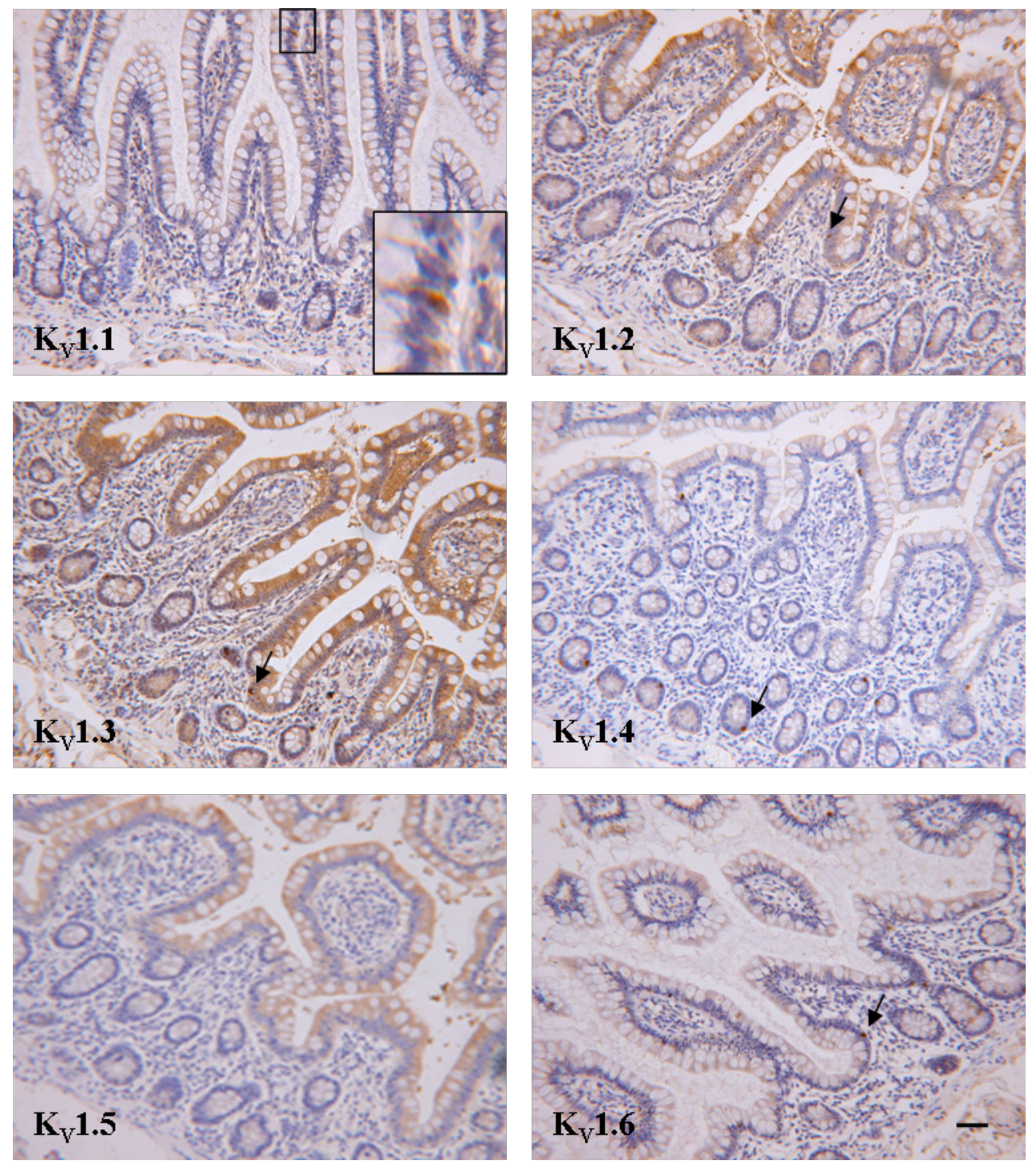

Figure 3.24: $K_{V} 1$ immunostaining of human ileal mucosa. $K_{V} 1$ subunits were localised to ileal enterocytes. Anti- $\mathrm{K}_{\mathrm{V}} 1.2$ and 1.3 antibodies strongly stained surface enterocytes and moderately stained crypt cells. $\mathrm{K}_{\mathrm{V}} 1.1$ and 1.5 appeared moderately expressed by ileal enterocytes. $\mathrm{K}_{\mathrm{V}} 1.4$ and $\mathrm{K}_{\mathrm{V}} 1.6$ appeared only weakly expressed in enterocytes. Enteroendocrine cells were intensely stained at their basal aspect by anti- $\mathrm{K}_{\mathrm{V}} 1.1$ (inset), 1.2, 1.3, 1.4 and 1.6 (arrows). Lamina propria and muscularis mucosa were negative. Scale bar $=50 \mu \mathrm{m}$. 

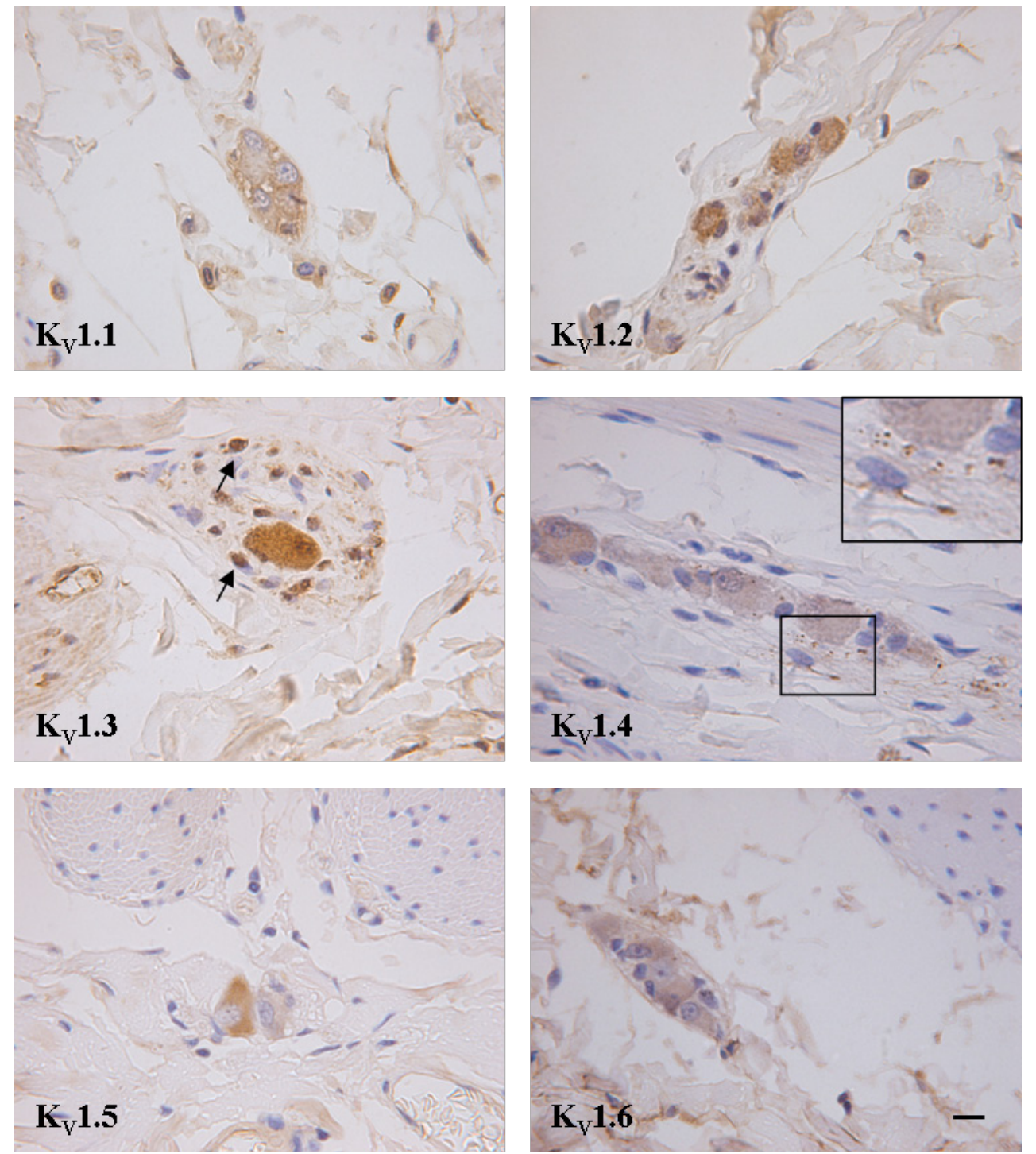

Figure 3.25: $K_{V} 1$ immunostaining of ileal submucosal ganglia. $K_{V} 1.1$ was localised to the myenteric neuropil, whereas $\mathrm{K}_{\mathrm{V}} 1.2$ was limited to cell bodies only. $\mathrm{K}_{\mathrm{V}} 1.3$ exhibited strong nuclear (arrows) and cell body cytoplasmic staining. $\mathrm{K}_{\mathrm{V}} 1.4$ was present in fine puncta adjacent to certain cell bodies (inset) and towards ganglia periphery and weakly in cell bodies. Cell bodies strongly stained for $\mathrm{K}_{\mathrm{V}} 1.5$ whereas $\mathrm{K}_{\mathrm{V}} 1.6$ was only weakly present. Scale $\mathrm{bar}=10 \mu \mathrm{m}$. 

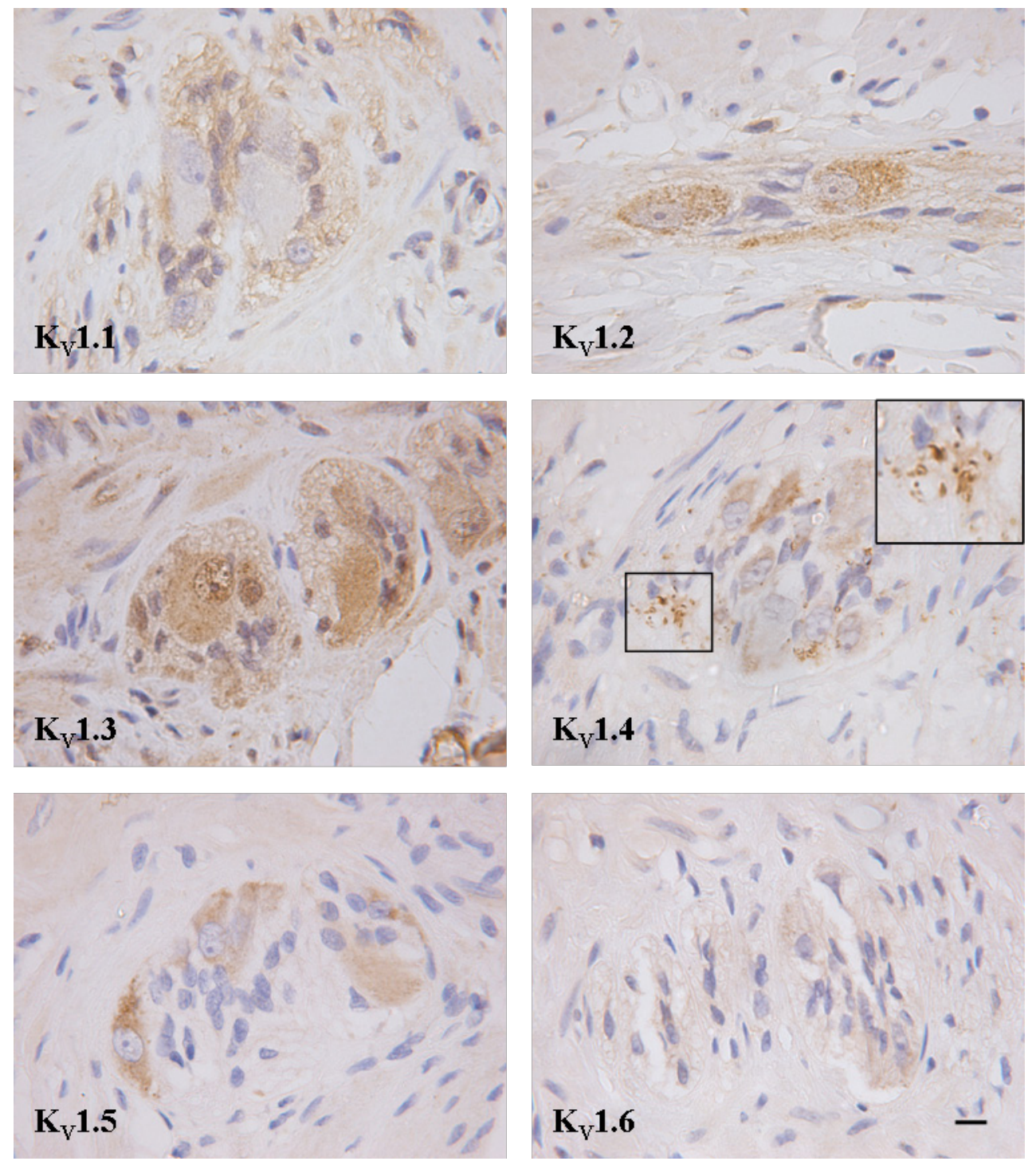

Figure 3.26: $K_{V} 1$ immunostaining of ileal myenteric ganglia. The neuropil was moderately positive for $\mathrm{K}_{\mathrm{V}} 1.1 . \mathrm{K}_{\mathrm{V}} 1.2$ appeared highly expressed by some myenteric cells bodies and moderately by others. $\mathrm{K}_{\mathrm{V}} 1.3$ exhibited nuclear staining with moderate staining of cell body cytoplasm. $\mathrm{K}_{\mathrm{V}} 1.4$ expression appeared restricted to discrete puncta (inset) with occasional weak cell body staining. $\mathrm{K}_{\mathrm{V}} 1.5$ moderately stained cell bodies. Ganglia were negative for $\mathrm{K}_{\mathrm{V}} 1.6$. Scale bar $=10 \mu \mathrm{m}$. 


\subsubsection{COLON}

\section{Colonic mucosa}

The gradient of $\mathrm{K}_{\mathrm{V}} 1$ expression in surface compared with deep epithelial cells was most pronounced in the colon (Figure 3.27). Surface enterocytes and goblet cells appeared moderately to strongly positive for all six subunits. Crypt enterocytes appeared largely negative except for enteroendocrine cells which displayed immunoreactivity for $\mathrm{K}_{\mathrm{V}} 1.1$, 1.4 and 1.6 (Figure 3.27). Lamina propria and muscularis mucosa were negative.

\section{Colonic neurons}

Submucosal ganglion cell bodies and neuropil were moderately immunostained with anti$\mathrm{K}_{\mathrm{V}}$ 1.1. In myenteric ganglia this appeared largely restricted to the neuropil where only selected cell bodies appeared positive. $\mathrm{K}_{\mathrm{V}} 1.2$ immunostaining was selectively localised to cell bodies which were moderately immunopositive in submucosal ganglia. Selected myenteric ganglion cell bodies were strongly $\mathrm{K}_{\mathrm{V}} 1.2$ immunopositive whereas others appeared negative. $\mathrm{K}_{\mathrm{V}} 1.3$ localised to neuronal cell bodies of both plexuses, more strongly in the submucosal, with the additional observation of nuclear staining. Submucosal cell bodies were moderately positive for $\mathrm{K}_{\mathrm{V}} 1.4$ whereas myenteric cells displayed only weak immunostaining. Punctate staining of $\mathrm{K}_{\mathrm{V}} 1.4$ containing structures observed in gastric, jejunal and ileal ganglia were not seen in the colon. In both plexuses $\mathrm{K}_{\mathrm{V}} 1.4$ was localised to cell bodies, moderately in submucosal and weakly in myenteric. $\mathrm{K}_{\mathrm{V}} 1.5$ immunostaining was strongly present in submucosal and selected myenteric cell bodies. $\mathrm{K}_{\mathrm{V}} 1.6$ appeared to be moderately expressed by submucosal cell bodies but only weakly expressed in myenteric cell bodies and neuropil (Figures 3.28. and 3.29, respectively). Colonic smooth muscle appeared negative for $\mathrm{K}_{\mathrm{V}} 1$ subunit expression (not shown). 

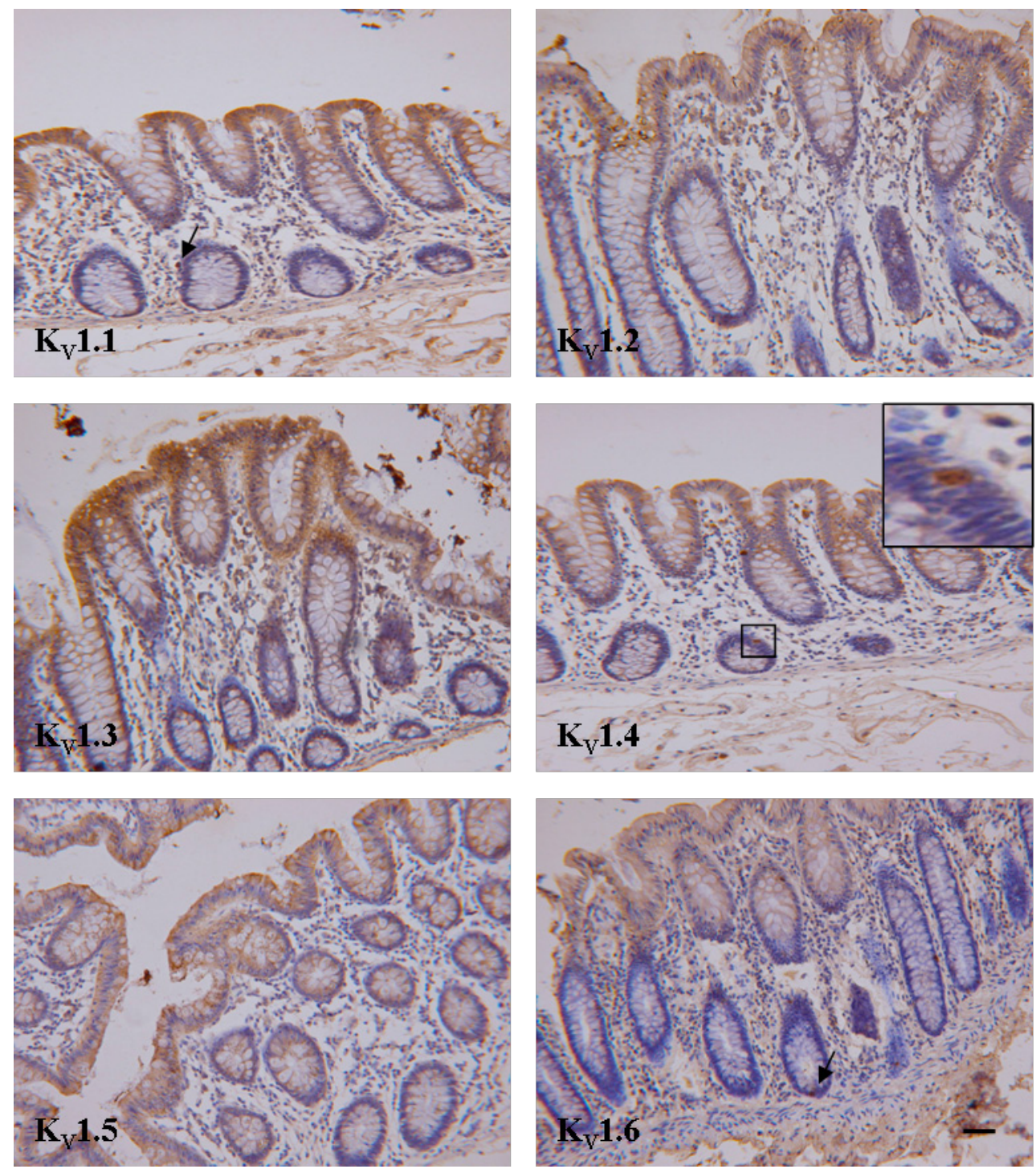

Figure 3.27: $K_{V} 1$ immunostaining of colonic mucosa. Surface enterocytes appeared positive for $\mathrm{K}_{\mathrm{V}} 1.1-1.6$ channel expression, whereas deep epithelial cells appeared negative. $\mathrm{K}_{\mathrm{V}} 1.1,1.4$ and 1.6 were also present in the basolateral membrane of a subset of cells (presumed enteroendocrine, inset and arrows). Muscularis mucosa immunostaining was not above background levels. Scale bar $=50 \mu \mathrm{m}$. 

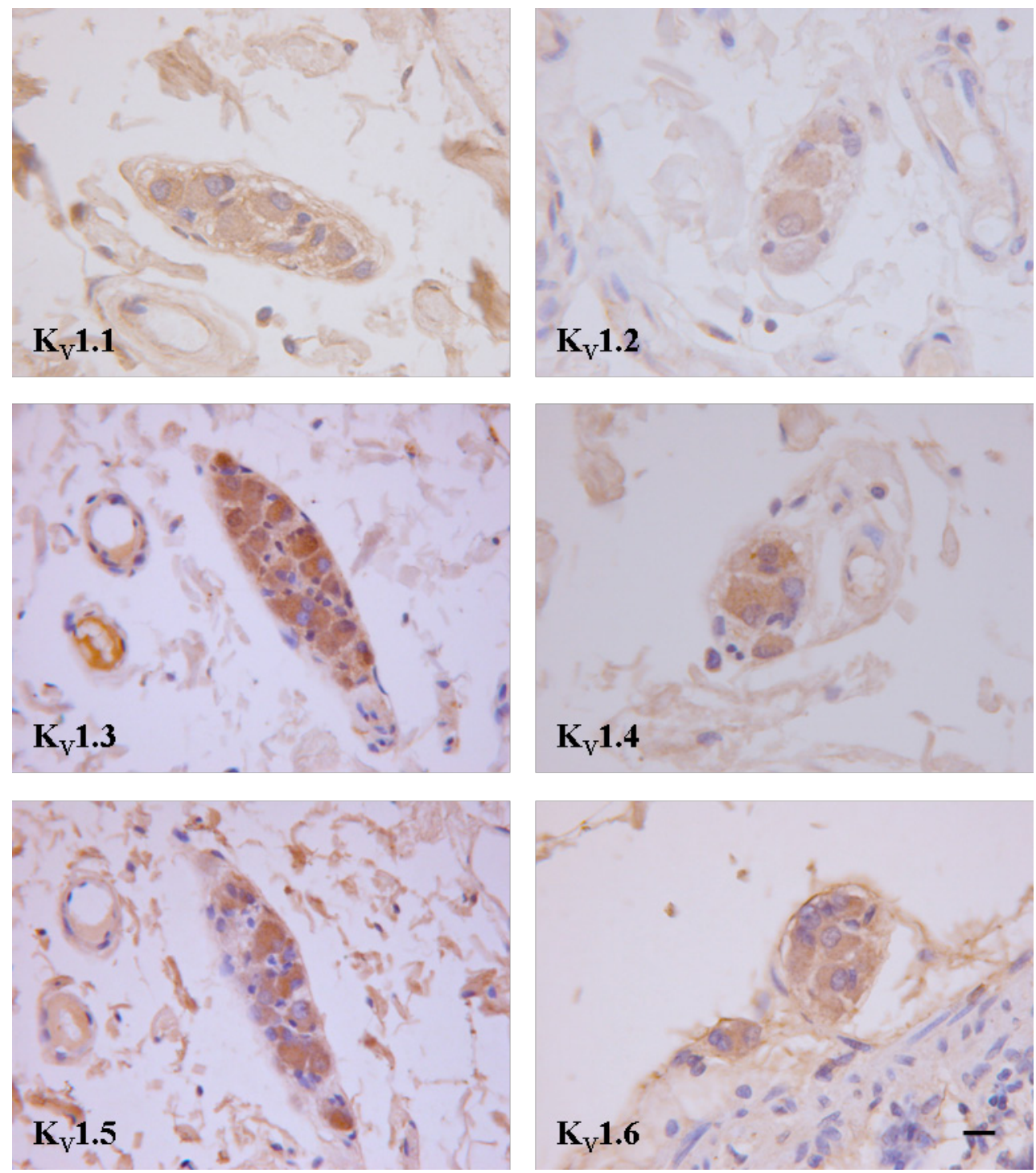

Figure 3.28: $K_{V} 1$ immunostaining of colonic submucosal ganglia. $K_{V} 1.1$ immunostaining was present in both neuronal cell bodies and the surrounding neuropil whereas $\mathrm{K}_{\mathrm{V}} 1.2$ appeared restricted to cell bodies. Neuronal cell bodies appeared to strongly stain for $\mathrm{K}_{\mathrm{V}} 1.3$, both cytoplasmic and nuclear. Immunostaining for $\mathrm{K}_{\mathrm{V}} 1.4$ and 1.6 was moderately strong in cell bodies. $\mathrm{K}_{\mathrm{V}} 1.5$ immunostaining was strong. Scale bar $=10 \mu \mathrm{m}$. 

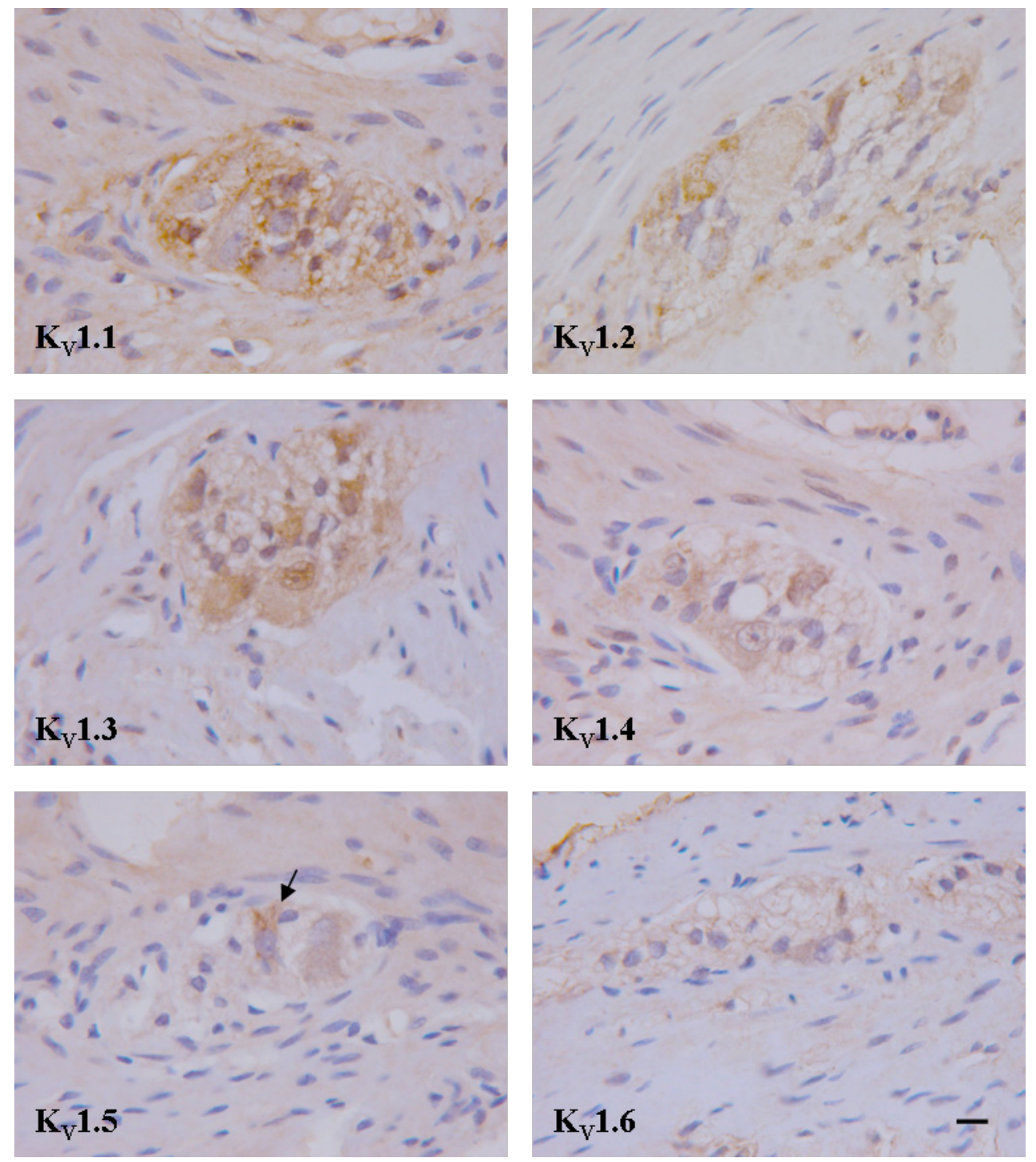

Figure 3.29: $K_{V} 1$ immunostaining of colonic myenteric ganglia. $K_{V} 1.1$ was largely confined to the neuropil. Selected neuronal cell bodies appeared to strongly stain for $\mathrm{K}_{\mathrm{V}} 1.2,1.3$ and 1.5 $\mathrm{K}_{\mathrm{V}} 1.1$ was also present in the neuropil. $\mathrm{K}_{\mathrm{V}} 1.3$ stained both cytoplasm and cell nuclei. $\mathrm{K}_{\mathrm{V}} 1.4$ and $\mathrm{K}_{\mathrm{V}} 1.6$ weakly immunostained the neuropil and cell bodies. Scale bar $=10 \mu \mathrm{m}$. 


\subsubsection{KVI.4 LOCALISATION IN HUMAN GASTRIC MYENTERIC PLEXUS}

Following the identification of a unique punctate distribution of $\mathrm{K}_{\mathrm{V}} 1.4$ in enteric ganglia, the distribution of this subunit was further investigated using double-labelling immunofluorescent staining in the gastric myenteric plexus.

\section{$K_{V} 1.4$ immunofluorescent staining}

In the same manner as that observed with DAB immunohistochemistry, tissue immunofluorescently stained for $\mathrm{K}_{\mathrm{V}} 1.4$ exhibited multiple fine puncta throughout ganglia, often clustered around the ganglion cell bodies and ganglia periphery. Immunofluorescent staining revealed more $\mathrm{K}_{\mathrm{V}} 1.4$ containing structures than the DAB method, which, in addition to simple puncta also defined small vesicular structures (Figure 3.30).

\section{Neurofilament and $K_{V} 1.4$}

Neurofilament immunostaining was dense throughout the myenteric plexus, identifying axons and some cell bodies of myenteric ganglia. Where axons were cut transversely, NF staining appeared punctuate, although as larger patches than $\mathrm{K}_{\mathrm{V}} 1.4$ puncta. $\mathrm{K}_{\mathrm{V}} 1.4$ immunoreactivity was often closely associated with NF and occasionally localised to the periphery of NF-positive structures suggesting this subunit may be localised to a subpopulation of axons (Figure 3.30).

Synaptophysin and $K_{V} 1.4$

Synaptophysin immunoreactivity was present throughout ganglia, concentrated at ganglia peripheries and surrounding cell bodies. Double-labelling with anti- $\mathrm{K}_{\mathrm{V}} 1.4$ revealed frequent but not universal overlap with $\mathrm{K}_{\mathrm{V}} 1.4$ puncta, suggesting channels are located at a subpopulation of synaptic terminals (Figure 3.30). 

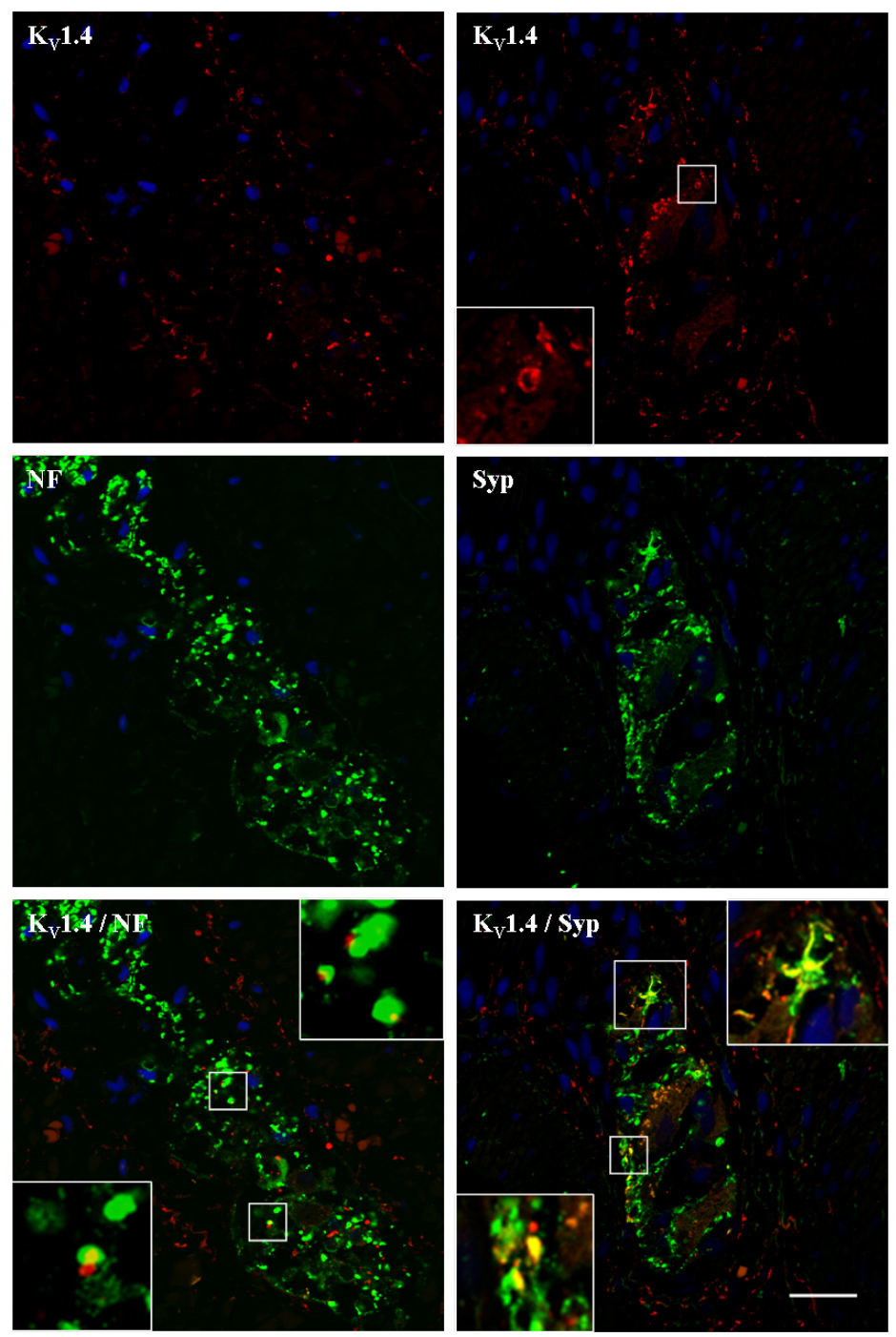

Figure 3.30: Co-localisation of $K_{V} 1.4$ and neuronal markers in the gastric myenteric

plexus. $\mathrm{K}_{\mathrm{V}} 1.4$ exhibited punctate distribution throughout myenteric ganglia and also occasional structures which appeared vesicular. Left column: NF immunostaining revealed multiple large puncta within ganglia. Occasional overlap was seen between $\mathrm{K}_{\mathrm{V}} 1.4$ and NF puncta (inset), suggesting localisation to a subpopulation of axons and / or dendrites. Right column: Synaptophysin immunoreactivity was also punctate, surrounding cell bodies, and also defined larger, branching areas, presumably representing the divergence of axonal terminals. $\mathrm{K}_{\mathrm{V}} 1.4$ and synaptophysin immunostaining frequently overlapped (inset), suggesting localisation to a subpopulation of synaptic terminals. Syp = synaptophysin, NF $=$ neurofilament. Scale bar $=20 \mu \mathrm{m}$. 


\section{DISCUSSION}

\section{4}

\subsection{ORIGINAL PROJECT AIMS}

4. To investigate the presence of autoantibodies directed to enteric ion channels in patients with neuromuscular disorders of the gut leading to severe dysmotility.

5. To investigate whether autoantibodies (if present) are pathogenic.

6. To further elucidate the profile of expressed ion channels in the human enteric neuromusculature.

\subsection{SUMMARY OF MAIN RESULTS}

The main results of each section of this thesis will be highlighted and discussed in relation to existing studies relevant to this field.

Finding 1: Humoral autoimmunity against currently recognised neuronal antigens is not a common cause of primary GINMD

The presence of circulating autoantibodies against a number of neuronal antigens was determined in 21 patients with primary achalasia and 27 patients with severe intestinal dysmotility (SID) using well validated radioimmunoprecipitation assays. The targets of interest were voltage- and ligand-gated ion channels and a neuronal enzyme which are 
known antigens of pathogenic autoantibodies in autoimmune peripheral and central neurological diseases. These were group 1 voltage-gated potassium channels (VGKC / $\mathrm{K}_{\mathrm{V}} 1$; acquired neuromyotonia, and limbic encephalitis), voltage-gated calcium channels (VGCC; Lambert-Eaton myasthenic syndrome), ganglionic $\alpha 3$ nicotinic acetylcholine receptors ( $\alpha 3 \mathrm{nAChR}$; autoimmune autonomic neuropathy) and glutamic acid decarboxylase (GAD; epilepsy and cerebellar ataxia). Sera from patients with SID were also screened for the presence of anti-neuronal antibodies (ANAs) which are frequently present in dysmotility secondary to neoplasia. All patients were negative for anti-channel and anti-neuronal antibodies.

\section{Study limitations}

It is possible the negativity of our screening could be due to inadequate assay sensitivity or specificity. This is unlikely as the radioimmunoprecipitation assays employed are wellvalidated (Apiwattanakul et al. 2010) and are routinely used in clinical diagnosis. Methodological error is also an unlikely cause of our negative findings as disease control samples included in each assay were consistently positive in the expected titre ranges and each assay was repeated at least twice to confirm results. It is more likely that the converse is true, i.e. the approach taken was too specific and that we might, with hindsight, have used a broader although less specific screening method before embarking on the detailed assays used (see below). Thus, a limitation of our study was the small number of targets screened for. We included antigens which are proven pathogenic targets in peripheral neuromuscular and central nervous system (CNS) diseases, autoantibodies against which have also been previously identified in patients with a secondary GINMD, particularly in association with cancer, and in small numbers of patients with a primary GINMD. Although we included almost all of these antigens, due to time constraints, some autoantibodies were not screened. These included N-type VGCCs, which have been found in primary achalasia, delayed gastric emptying and slow transit constipation (Dhamija et al. 2008; Kraichely et al. 2009). It is important to point 
out that the number of targets for autoantibodies is continually growing, an example being previously 'seronegative' cases of myasthenia gravis now recognised as positive for autoantibodies against a muscle-specific receptor tyrosine kinase (MuSK) (Hoch et al. 2001; Wolfe et al. 2008). The number of assays available is far behind the number of potential targets, being limited, in the case of radioimmunoprecipitation assays, by the availability of specific toxins to label the channel of interest.

A screening tool which has been used extensively in the investigation of humoral autoimmunity in achalasia is indirect immunofluorescent staining of the target tissue using patient serum (Storch et al. 1995; Verne et al. 1997; Moses et al. 2003). Whilst a positive result does not provide limited information regarding the specific autoantigen, it is a simple means by which evidence for or against 'anti-gut' autoantibodies can be gathered before proceeding to more complex assays to determine the antigen identity. An advantage of this method is that the specificity of the IgG for the gut and gut region may be determined. However, caution should be applied in interpreting results. False-positive results could arise due to non-specific binding, which without a specific antigen in mind is difficult to control for. False-negative results may occur as consequence variable antigen availability following tissue processing and due to temporal or sub-regional variation in antigen expression. There is also a precedent in the study of achalasia for HLA-typing. Certain alleles have been shown to be significantly associated with achalasia and also in this regard with the presence of anti-neuronal antibodies (Ruiz-deLeon et al. 2002), although a correlation with antibodies may not be true for every population (Latiano et al. 2006). Nevertheless, HLA-typing of our achalasia patients may have allowed us to better select those in whom the presence of anti-neuronal antibodies is more likely. Identification of a specific HLA allelic association in pseudo-obstruction and enteric dysmotility would lend further support to an autoimmune mechanism, but to date there is no precedent for this. 


\section{Significance and interpretations}

Primary GINMD are relatively rare disorders and findings of anti-neuronal or antichannel autoantibodies have previously been restricted to case-reports or small cohorts. The number of cases screened in our study ( 21 achalasia and 27 SID) represents one of the largest cohorts of primary GINMD studied in this manner to date. A similar study (Dhamija et al. 2008) screened 29 patients with GINMD affecting one or more levels of the gut. This study was designed to investigate the profile of autoantibodies useful in the diagnosis of suspected 'autoimmune gastrointestinal dysmotility' (AGID) as all patients had been selected due to the proven existence of one or more autoantibody. AGID represents a group of patients who have a limited form of dysautonomia with prominent GI symptoms of hyper- or hypo-motility and one or more autoantibodies against cellsurface cation channels and includes GI dysmotility secondary to neoplasia, diabetes mellitus and hypothyroidism. It may also occur idiopathically as a manifestation of autoimmune autonomic neuropathies (Lennon et al. 1991; Smith et al. 1997; Vernino et al. 2000). Although the study by Dhamija et al. screened 29 cases, only 8 of these could be considered as primary GINMD, the others having neoplasia and / or other disease. Anti-P/Q-type VGCC antibodies were found in a case of primary achalasia, anti-N-type VGCC in a patient with slow small intestinal transit, anti-muscle nAChR in another case of primary achalasia, anti-ganglionic $\alpha 3 \mathrm{nAChR}$ in two patients with delayed gastric emptying and in a patient with delayed small intestinal and colonic transit, who also had anti-VGKC antibodies, and finally anti-VGKC antibodies in a patient with delayed colonic transit. Whilst useful in defining which autoantibodies are indicative of AGID, the selectivity bias and heterogeneity of findings means that the results of this study cannot be generalised to unexplained GINMD. All of our patients were selected on the basis of a confirmed primary GINMD and had not previously been investigated for antichannel autoimmunity. All patients with SID were subsequently confirmed to be negative for anti-neuronal antibodies (ANAs) commonly associated with neoplasia (anti-Hu, Yo and Ri). In light of the results of Dhamija et al. it is surprising that none of our patients 
had detectable autoantibodies. An important reason for this could be the chronicity of the disorders in our patient groups. Dhamija et al. do not provide information regarding symptom duration in their patients, however the median duration of follow-up was only 25 months (range 2-127). Two studies have previously screened unselected patients with primary GI dysmotility. The first of these detected high titres of anti-VGKC antibodies in two of eleven patients with colonic slow transit constipation, one gave a 6 year history, the other had had symptoms for only 1 year (Knowles et al. 2002). The second study screened 33 patients. The median duration of dysmotility symptoms for this group was 18 years (range 5-60). Two patients with a 9 and 12 year history of diarrhoea-predominant IBS were seropositive for anti-VGKC and anti- $\alpha 3 \mathrm{nAChR}$ antibodies respectively. In both of these cases a full-thickness jejuna biopsy revealed an inflammatory neuropathic process. Interestingly, none of 4 patients with enteric dysmotility, 8 patients with intestinal pseudo-obstruction and 9 patients with slow transit constipation had detectable autoantibodies (Tornblom et al. 2007). The median duration of symptoms of the SID patients of our study, 10 years (range 3-40 years) was similar to that of Tornblom et al. It is possible then that autoantibodies in primary GINMD are an early phenomenon which subsequently resolve but lead to chronic disease due to activation of lymphocytes, macrophages/killer cells producing permanent damage. In practise this is difficult to study as the best characterised autoimmune disorders are treated by immunomodulation. However, there is further evidence in support of this. Anti-VGKC antibodies have retrospectively been observed to be monophasic with levels falling over time without immunomodulatory therapy (Buckley et al. 2001). Anti-VGKC, -GAD and - N-methylD-aspartate receptor (NMDAR) antibodies associated with non-paraneoplastic limbic encephalitis appear to be highest during the early stage of disease and early treatments appear to produce optimal clinical outcomes (Irani et al. 2010) Rassmussen's encephalitis which has been associated with anti-glutamate receptor antibodies also responds well to immunotherapy in the early course of the disease, but as the disease progresses this is less effective (Bethan Lang, personal correspondence). Furthermore, in anti-GAD antibody 
associated epilepsy, antibody titres are much higher at presentation than seen in chronic disease (Bethan Lang, unpublished observation).

Regarding primary achalasia, a study of a large group (70) of unselected patients revealed a remarkably high prevalence of anti-neuronal antibodies, $25.7 \%$ having one or more of anti-skeletal muscle AChR or striational, neuronal voltage-gated cation channel (potassium channel or N-type calcium channel) or GAD antibodies. In addition, 21\% had anti-GAD antibodies. The mean number of years from diagnosis to sera collection in this group was only 5 years (Kraichely et al. 2009). The mean number of years from diagnosis to sera collection in our achalasia group was 6 years. However, this does not necessarily correlate with disease duration as some of our patients, even at first investigation, were noted to have a manometric pattern characteristic of established achalasia. Data regarding stage of disease at diagnosis in the study of Kraichely et al. are not provided. It should be mentioned that none of the positive anti-GAD sera, which were detected using an indirect immunofluorescence assay, were confirmed using the radioimmunoprecipitation assay to confirm specificity (Saiz et al. 2008).

Interpreting our results in the light of these previous studies we may tentatively speculate that the failure to detect anti-channel autoantibodies in our GINMD patients may be due to chronicity where antibodies which may present in early disease 'burn-out', leaving a more chronic, cell-mediated immune process. To test this hypothesis it would be desirable to study patients in the early stages of disease and follow-up with a longitudinal study. Dhamija et al. report a moderate to dramatic improvement in gastrointestinal symptoms in 4 out of 4 seropositive patients treated with immunotherapy. 


\section{Summary box: Humoral autoimmunity in primary GINMD}

Previous knowledge

- Non-specific anti-neuronal autoantibodies present in significant number

- Anti-channel (VGKC, VGCC, nAChR) antibodies detected in a few cases and may represent a limited form of dysautonomia (AGID)

- Anti-GAD present in significant number of primary achalasia

This study

- Anti-channel antibodies not associated with chronic primary GINMD

- Anti-GAD antibodies not detected in neurologically significant titres in primary achalasia

\section{Finding 2: Autoantibodies which directly bind the $M_{2}$ muscarinic acetylcholine receptor were not detected in chagasic patients.}

A large body of data from in vitro pharmacological experiments supports the existence of circulating autoantibodies in the sera of patients with Chagas' disease and GI involvement, which, in GI smooth muscle, produce effects mediated by activation of $\mathrm{M}_{2}$ mAChRs. However, the only evidence for direct binding to the receptor previously came from ELISA assays which employ a synthetic peptide representing only a part of the receptor. We designed a cell-based assay to screen for the presence of anti- $\mathrm{M}_{2} \mathrm{mAChR}$ autoantibodies to clarify whether such autoantibodies are able to directly bind to the receptor. We did not detect IgG in the sera of 57 chagasic patients capable of directly binding the $\mathrm{M}_{2} \mathrm{mAChR}$. Thus anti- $\mathrm{M}_{2} \mathrm{mAChR}$ autoantibodies, if present in this cohort of patients, do not exert their effects by direct receptor binding. 


\section{Study limitations}

The negativity of our assay could be explained by the fact it was a newly designed assay and was not sufficiently optimised to detect autoantibodies. Control measures were taken however to ensure viability. These controls have previously been employed in the successful optimisation of other cell-based assays used for this purpose. Firstly, transfection efficiency was checked using the well validated method of GFP transfection to produce auto-fluorescent cells (Modarressi et al. 2004). This confirmed our method of transfection was robust. Secondly, successful expression of $\mathrm{M}_{2} \mathrm{mAChR}$ was confirmed by immunofluorescently staining transfected cells with a commercial anti- $\mathrm{M}_{2} \mathrm{mAChR}$ monoclonal antibody (Leite et al. 2008). It should be noted that only commercial antibodies raised against intracellular epitopes of the receptor were available at this time and as such it was necessary to permeabilise the transfected cells prior to antibody incubation. Whilst not directly comparable to subsequent staining using patient serum on intact cell membranes, this control demonstrated that the receptor protein was successfully expressed by transfected cells and the pattern of staining, despite the permeabilisation process appeared to delineate a circumscribed cell border, suggesting the receptor was reaching the cell membrane. With these conditions satisfied one may feel confident in using the assay to screen sera for antibodies against the target in question. Indeed, this assay is increasingly becoming the assay of choice in detecting autoantibodies as it has been shown to be more sensitive than traditional radioimmunoprecipitation assays and is closer to the in vivo conditions where autoantibodies meet membrane bound, fully processed channels and receptors (Leite et al. 2008).

\section{Significance and interpretations}

The sufficiency of ELISA using a short, linear portion of a receptor to detect autoantibodies has been called into question (Vincent et al. 2010). Indeed this issue is highlighted by a similar study of GI dysmotility secondary to scleroderma (Goldblatt et 
al. 2002). Circulating autoantibodies which modify in vitro events associated with muscarinic antagonism have been detected in such patients, and have been postulated, as a result of indirect pharmacological evidence, to exert a functional effect, via the $\mathrm{M}_{3}$ mAChR. Furthermore, autoantibodies in the sera of patients with scleroderma and GI involvement have been shown to bind a synthetic $\mathrm{M}_{3} \mathrm{mAChR}$ peptide in ELISA. However, the authors of the in vitro study note in their discussion that functional these autoantibodies failed to bind mammalian cells transfected with the $\mathrm{M}_{3} \mathrm{mAChR}$ calling into question direct interaction with the receptor itself. Whilst the pharmacological evidence for $\mathrm{M}_{2} \mathrm{mAChR}$ activation in the presence of chagasic autoantibodies is stronger than for $\mathrm{M}_{3} \mathrm{mAChR}$ antagonism in scleroderma, the results of our cell-based assay suggest that anti-muscarinic autoantibodies capable of directly binding the $\mathrm{M}_{2} \mathrm{mAChR}$ are not always present in a significant number of patients with Chagas' disease and GI involvement, as currently claimed.

An important consideration in interpreting our results is the origin of our cohort of serum samples which came from individuals living in the north-east of Brazil. All previous studies relevant to this subject have examined the effects of sera collected from patients residing in Argentina (specifically in the Beunos Aires area). This is significant as clinical manifestations of chronic Chagas' disease are distinct in different geographical regions, megaviscera being more prominent in the southern countries of South America. It is now known that Trypanosoma cruzi (T.cruzi) is a heterogeneous organism, consisting of several strains (Miles et al. 2009), and whilst host factors must also play a role, differences in infecting genotypes appear to govern chronic disease sequalae (Figure 4.1). It is then likely that the patients in the present cohort were infected by a genotypically different organism to those studied previously, and although patients studied here and previously had GI involvement, the failure to detect anti- $\mathrm{M}_{2} \mathrm{mAChR}$ autoantibodies in our cohort may be due to a different immune response which is consequent of a dissimilar infective event. To help answer the question of whether the 
present cohort have autoantibodies which activate muscarinic pathways without binding receptors directly or have another mechanism of action, we proceeded to investigate the functional effects of the sera from our cohort on GI smooth muscle.
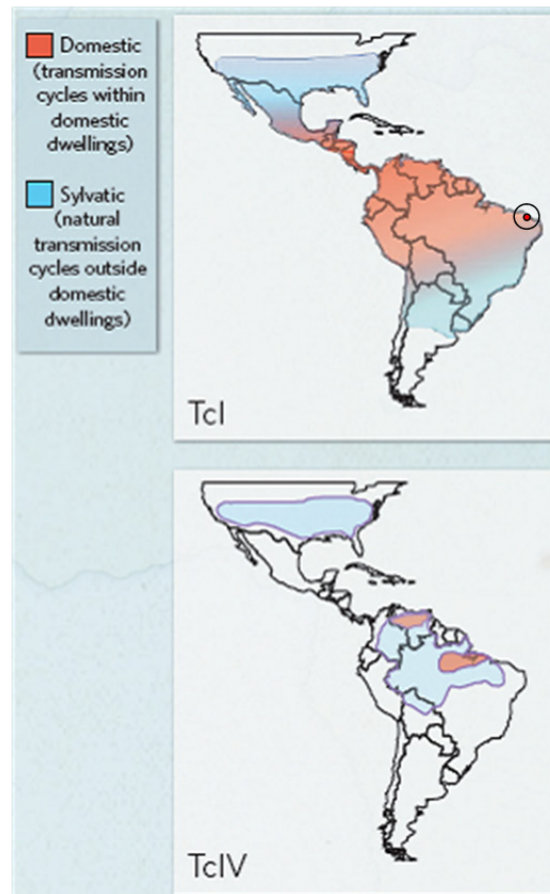
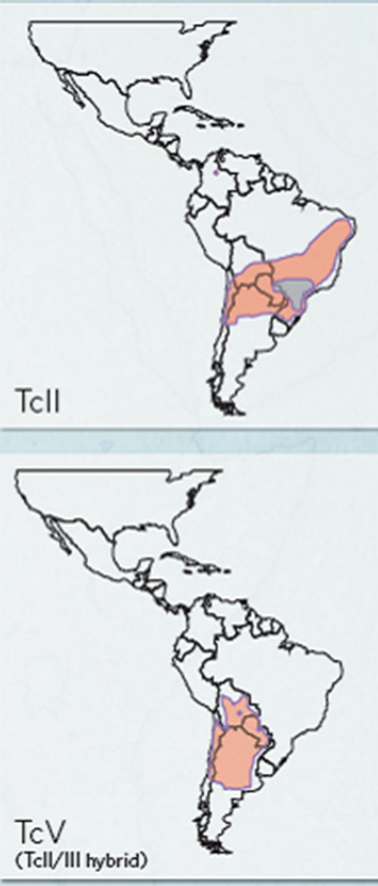
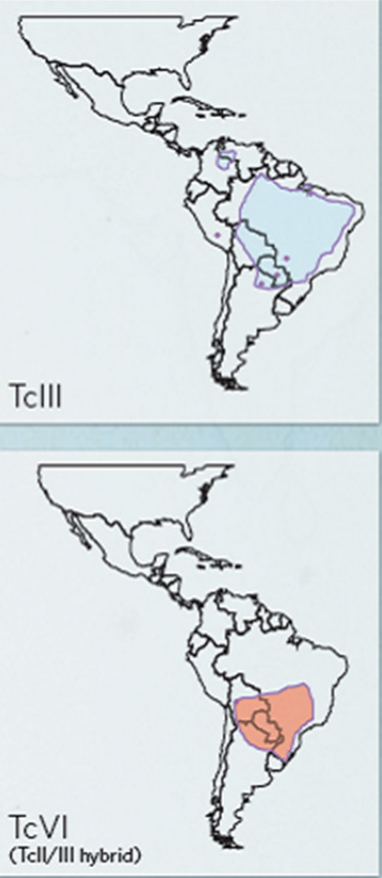

Figure 4.1: Geographical distributions of T.cruzi lineages (Te I - VI). Sera analysed in this thesis were taken from patients residing in and around the city of Fortaleza, in the north-east of Brazil (indicated in the upper left panel). It is possible that individuals in this cohort were infected with any of four strains of T.cruzi (Tc I - IV), which may be transmitted via domestic or sylvatic triatomines (haematophagous insect vectors, a subfamily of the genus Reduvius). Adapted by permission from Macmillan Publishers Ltd: Nature (465, S8-9), copyright (2010). 


\section{Summary box: Anti-muscarinic humoral autoimmunity in Chagas' disease}

Previous knowledge

- Autoantibodies present in high proportion of individuals with Chagas' disease are capable of modulating muscarinic GI neuromuscular activity

- Autoantibodies bind synthetic $\mathrm{M}_{2}$ peptide in ELISA suggesting direct receptor modulation

This study

- Autoantibodies capable of directly binding $\mathrm{M}_{2} \mathrm{mAChR}$ expressed by mammalian cells were not detected in patients with Chagas' disease suggesting an alternative mechanism of receptor activation or absence of anti- $\mathrm{M}_{2} \mathrm{mAChR}$ antibodies

Finding 3: Circulating factors present in patients with Chagas' disease modulate colonic smooth muscle contractility through non-cholinergic / non-nitrergic pathways

A number of studies have implicated chagasic IgG in activation of the $\mathrm{M}_{2}$ muscarinic cholinergic signalling pathway with subsequent end organ effects (Goin et al. 1994; Goin et al. 1994; Goin et al. 1997; Sterin-Borda et al. 1997; Hernandez et al. 2008; Ganzinelli et al. 2009) including an increase in GI smooth muscle tone (Goin et al. 1999; SterinBorda et al. 2001). It should be noted that the majority of these studies originate from a single research group, and all studies have used the same ELISA to detect anti- $\mathrm{M}_{2}$ $\mathrm{mAChR}$ autoantibodies. Regarding the investigation of serum effects on colonic smooth muscle tone, in vitro experiments have used muscle taken from either normal rat colon or human chagasic megacolon samples, reporting very similar responses to incubation with chagasic serum in both tissues. This result is curious as inter-species variability in colonic muscarinic receptor profile appears to exist (Gomez et al. 1992) and chagasic megacolon is characterised by extensive damage to the neuromuscular apparatus including 
significant inflammatory enteric neuronal degeneration, muscular inflammatory cell infiltration and muscular fibrosis (Corbett et al. 2001; da Silveira et al. 2007). Our cellbased assay designed to detect anti- $\mathrm{M}_{2} \mathrm{mAChR} \operatorname{IgG}$ failed to detect these autoantibodies in a cohort of Chagas' disease patients. We therefore undertook the investigation of the effect of chagasic serum on colonic smooth muscle taken from patients without Chagas' disease.

The overall observed effect was a decrease in baseline smooth muscle tension. This effect was maintained in the presence of the muscarinic antagonist atropine and the nitric oxide synthase inhibitor L-NAME, demonstrating that it was not dependent on muscarinic cholinergic receptor activation or nitric oxide production. This finding represents a novel chagasic serum effect on colonic smooth muscle contractility.

\section{Study limitations}

The most important limitation of our findings is the fact that we have investigated the effect of serum from only three patients with chagasic GI disease. For this reason we cannot generalise this finding. Work is on-going to characterise more samples. We did however investigate the effects of disease control serum (from patients with Chagas' disease but no GI involvement) and community healthy control serum (from other individuals living in north-eastern Brazil without Chagas' disease), neither of which showed a comparable effect on smooth muscle tone. It would have also been desirable to purify IgG from the sera to determine if this effect was antibody mediated or due to other circulating factors. However, previous work has shown almost identical effects of whole serum and purified IgG on cholinergic muscle responses (Goin et al. 1994). As this was only a preliminary study, we used a single concentration of sera. Work is also on-going to determine a concentration-dependent response which would add weight to the evidence for a direct pathogenic role. 


\section{Significance and interpretations}

Previous studies reported that the addition of $\operatorname{IgG}$ autoantibodies from patients with Chagas' disease to in vitro preparations of GI smooth muscle resulted in $\mathrm{M}_{2} \mathrm{mAChR}$ agonism. Initially the IgG were found to non-competitively replace the binding of the cholinergic agonist carbachol, producing partial receptor agonism in an allosteric manner (resulting from binding at a site other than the primary, orthosteric binding site). This effect was also blocked by the muscarinic antagonist atropine. More recent studies report that the same autoantibodies may mediate their effect by positive modulation of acetylcholine activity at the $\mathrm{M}_{2} \mathrm{mAChR}$ (Hernandez et al. 2008). Whatever the proposed mechanism of action, with the exception of the dominant excitatory activity in the lower oesophageal sphincter in achalasia, the pathologic relevance of this effect in chagasic GI disease, which is characterised by inflammatory neuro-degeneration and pathological dilatation, remains unclear.

Our findings are novel in three respects. Firstly, we have shown an effect of chagasic sera on non-infected human colon. Secondly, we have identified a relaxant effect of chagasic sera on colonic smooth muscle which may represent early stages in the development of a megacolon. Thirdly, we have shown that this effect is not dependent on muscarinic cholinergic receptor activation.

Previous studies only looked at smooth muscle under a resting tension which does not provide information regarding whether activity is mediated by an alteration in neuronal or muscular activity. We examined the effect of chagasic sera on GI smooth muscle under electrical field stimulation (EFS) which allows neuronally mediated changes in muscle tension to be observed whilst also monitoring the changes in baseline muscle tone. We found that muscle responses during EFS were unchanged following addition of chagasic sera suggesting that the reduction in tone was not secondary to an alteration in neuronal activity. This evidence was strengthened by the observation that the effect was not 
prevented by pre-incubation of muscle preparations with the voltage-gated sodium channel blocker tetrodotoxin, which prevents action potential generation (Narahashi et al. 1960; Kao 1964). Furthermore, we found that the effect was not dependent on production of nitric oxide, the principle neurotransmitter of inhibitory motor neurons, or mAChR activation. The decrease in baseline muscle tension is therefore likely to be the result of a direct effect on the muscle. A candidate target for mediation of such an effect is the Ltype voltage-gated calcium channel (VGCC), which is the predominant channel responsible for calcium entry into intestinal smooth muscle cells necessary for contraction (Farrugia et al. 1995; Liu et al. 2001). The L-type VGCC antagonist, nifedipine, has been shown to reduce the basal tone of human colonic circular muscle in vitro (Ghia et al. 2004). Furthermore, in isolated cardiac muscle cells, L-type $\mathrm{Ca}^{2+}$ current inhibition was observed in the presence of chagasic antibodies (Hernandez et al. 2003). Smooth muscle relaxation in response to serum factors may represent an early stage in the development of dysmotility which may progress to megacolon.

\section{Finding 4: Humoral anti-neuronal autoimmunity is present in some individuals with Chagas' disease}

Anti-channel autoantibodies were identified in patients with Chagas' disease. AntiVGCC antibodies were identified in one of 57 patients, although this patient had no secondary disease manifestations, antibody titre was comparable to LEMS disease control. Eight chagasic patients were found to have moderate titres of circulating antiVGKC antibodies. Of these, five had no chronic disease sequalae, one had achalasia and heart disease, one had achalasia only and one had colonic dysfunction. Low titre antiGAD antibodies were found in one patient with cardiac complications, although as previously stated, these are unlikely to have neurological significance 


\section{Significance and interpretations}

The finding of anti-VGCC and VGKC antibodies in Chagas' disease was unexpected and is the first time this has been described. Whilst the presence of these autoantibodies may occasionally be found in individuals with no overt neurological disease, the presence of anti-VGKC antibodies in $8(13.6 \%)$ of chagasic individuals is notable. The majority of these cases $(5 / 8)$ at the time of venepuncture had no sequalae of their infection. Of interest to this thesis the remaining three patients had GI diseae of oesophagus and / or colon. In the classical anti-VGKC-mediated diseases, such as neuromyotonia, GI features have not been especially reported (Isaacs 1961; Benatar 2000). However, anti-VGKC disorders are being increasingly recognised and three previous studies have described VGKC antibodies in relation to gut dysfunction. The first associated anti-VGKC autoantibodies with slow transit constipation arising de novo in adults (an uncommon presentation of a disorder which usually presents in childhood) (Knowles et al. 2002). Another describes VGKC antibodies occurring in a case of paraneoplastic (thymoma) associated intestinal pseudo-obstruction. Importantly, improvement in gastrointestinal symptoms following plasma exchange correlated with a decrease in VGKC antibody levels, suggesting for the first time a real pathological significance (Viallard et al. 2005). A study of 80 patients who were VGKC autoantibody positive found gastrointestinal symptoms in at least $12(17 \%)$ (the true figure may have been higher because some medical records studied retrospectively were incomplete) (Rueff et al. 2008). All patients included were identified on the basis of previous investigation for a suspected autoimmune neurological disorder, possibly paraneoplastic. It is not known whether any of these patients were initially referred because of isolated GI symptoms and what proportion were subsequently found to have an underlying neoplasm. However, this study further establishes an association between VGKC antibodies and GI symptoms.

Damage to the enteric nervous system is an established feature of chagasic GI disorders, and is characterised by neuronal degeneration and loss, connective tissue fibrosis, 
inflammatory cell infiltration, decreased numbers of interstial cells of Cajal and decreased enteric glia (Koberle 1968; Hagger et al. 2000; Iantorno et al. 2007). The production of autoantibodies in Chagas' disease has previously been attributed to molecular mimicry, where a cross-reactive immune response to a parasitic epitope and native antigen occurs, or due to a secondary immune response to tissue damage where 'cryptic' antigens which are normally hidden from the immune system are revealed (Kierszenbaum 2005). Our findings of anti-VGKC autoantibodies in patients with Chagas' disease, which is known to specifically lead to enteric neuronal pathology, together with our findings of $\mathrm{K}_{\mathrm{V}} 1$ channel expression in the gut would fit the hypothesis that these autoantibodies may occur as a result of tissue damage exposing antigens that are normally hidden to the immune system. In support of this was the identification of anti-VGKC antibodies in a patient with diarrhoea-predominant irritable bowel syndrome (D-IBS) which had begun after infectious enteritis. This patient was also found to have inflammatory cell infiltration (lymphocytic ganglionitis) and signs of enteric neuropathy (Tornblom et al. 2007). Against this is the fact that the majority of the patients in which we detected anti- $\mathrm{K}_{\mathrm{V}} 1$ channel antibodies had no disease sequalae, and others had only heart disease. However, even those without overt secondary disease would almost certainly have some parasite induced tissue damage that was perhaps at that time sub clinical (see Finding 5 for further discussion).

\section{Summary box: Anti-channel humoral autoimmunity in Chagas' disease}

Previous knowledge

- No previous description of anti-channel autoantibodies in Chagas' disease

This study

- $8 / 59(13.6 \%)$ patients positive for anti-VGKC autoantibodies but variable clinical phenotype 
Finding 5: Shaker-related voltage-gated potassium channels are expressed by the human gastrointestinal neuromuscular apparatus.

Due to the increasing evidence of anti-VGKC autoimmunity in certain types of GI dysfunction we set out to investigate the presence of these channels in the GI neuromuscular apparatus. The confirmation of their expression in the human GI tract has potential physiological significance and in the context of this and other studies, pathogenic implications.

Due to the specificity of $\alpha$-dendrotoxin, anti-VGKC radioimmunoprecipitation assays screen for autoantibodies against $\mathrm{K}_{\mathrm{V}} 1.1,1.2$ and 1.6 channel subunits (Stuhmer et al. 1989; Grissmer et al. 1994). However, due to the assembly of heteromultimeric channels (MacKinnon 1991; Parcej et al. 1992) and the heterogeny of diseases associated with anti-VGKC autoimmunity (Rueff et al. 2008), it is likely that other members of this subfamily also have pathogenic importance. Therefore, we investigated the expression of six members of the $K_{V} 1$ subfamily $\left(K_{V} 1.1-1.6\right)$ in all layers of the human oesophagus and gastrointestinal tract immunohistochemically. The results showed that $\mathrm{K}_{\mathrm{V}} 1$ channels are abundant throughout the human gastrointestinal tract and are expressed by epithelial cells and enteric neurons of the myenteric and submucosal plexi in a regionally specific pattern.

Epithelial $\mathrm{K}_{V} 1$ channel subunit expression varied according to the region studied. Oesophageal squamous epithelial cells were not immunopositive for any of the six $K_{V} 1$ channel subunit. In gastric epithelium $\mathrm{K}_{\mathrm{V}} 1$ expression was restricted to chief cells which are responsible for production and secretion of pepsinogen. Jejunal, ileal and colonic enterocytes expressed $K_{V} 1$ subunits to varying degrees, the concentration of channels appearing to be greatest in mature enterocytes towards villi tips and colonic surface epithelium with relatively little expression in the crypts. In addition, cells identified by 
H\&E appearance as enteroendocrine expressed a high abundance of channels in their basal aspect.

Within the enteric nervous system each $\mathrm{K}_{\mathrm{V}} 1$ channel subunit exhibited a stereotypical pattern of distribution, localising specifically to neuronal cell bodies and / or ganglia neuropil, and these patterns were largely consistent throughout the gut. Of the six subunits studied, $\mathrm{K}_{\mathrm{V}} 1.4$ was especially notable for its distinctive punctate distribution within enteric ganglia and this was further investigated using double-labelled immunofluorescent staining (see Finding 6).

\section{Study limitations}

This study was dependent on antibody specificity and, as with any immunohistochemical investigation, results could be due to non-specific tissue binding. This possibility is unlikely as we first confirmed that our antibodies stained cerebellar tissue in previously described patterns (Veh et al. 1995; Chung et al. 2001). These measures confirmed that the antibodies used were specific for these channels. The expression of cell surface proteins may be temporal, altering according to internal or external circumstances, and may also differ between individuals. It is therefore necessary to confirm findings in a number of different cases in order to describe 'normality'. For each gut region we studied $\mathrm{K}_{\mathrm{V}} 1$ channel distribution in at least five different individuals and found our results to be consistent. A limitation of this however was the source of tissue samples. The availability of disease-free tissue is, for obvious reasons, limited. The majority of tissue was obtained from cancer resection margins. Whilst all sections were histologically normal, with no evidence of cancer or inflammation, the possibility that channel expression is altered secondary to such disease processes cannot be ruled out. However, the difficulties inherent in studying normal human microanatomy mean that these results represent a significant step forward in mapping ion channel distribution in the human gut. 


\section{Significance and interpretations}

VGKC channels are involved in numerous processes in both excitable and non-excitable cells, but are best known in relation to setting resting membrane potentials, regulating cellular excitability and synaptic transmission. The $\mathrm{K}_{\mathrm{V}} 1$ or Shaker-related subfamily is the most extensively studied and the distribution of these channels in the CNS and periphery in relation to their role in normal function and disease states is fairly well elucidated (Beckh et al. 1990; Veh et al. 1995; Coleman et al. 1999; Chung et al. 2001; Glazebrook et al. 2002). In comparison, knowledge regarding $\mathrm{K}_{\mathrm{V}} 1$ channel expression in the GI tract was limited. Studies of small mammals had begun to identify $\mathrm{K}_{V}$ (including $\mathrm{K}_{\mathrm{V}} 1$ ) currents associated with epithelial cell maturation, migration and nutrient absorption (McDaniel et al. 2001; Rao et al. 2002). Cells from the IEC-6 cell line derived from rat intestinal enterocytes express $\mathrm{K}_{\mathrm{V}} 1.1,1.2,1.4,1.5$ and $1.6 \mathrm{mRNA}$ (McDaniel et al. 2001). Furthermore, as these cells migrate and differentiate towards a mature phenotype the expression of $\mathrm{K}_{\mathrm{V}} 1.1$ and 1.5 increases (Rao et al. 2002). Our finding of $\mathrm{K}_{\mathrm{V}} 1$ channel expression in mature, differentiated enterocytes of the human GI tract is in agreement with these findings and further suggests that increased $\mathrm{K}_{\mathrm{V}} 1$ channel expression occurs with human enterocyte maturation.

Modulation of enteric motor nerve excitability (Suarez-Kurtz et al. 1999; Vianna-Jorge et al. 2000) and generation of smooth muscle 'slow-wave' contractions (Thornbury et al. 1992; Thornbury et al. 1992; Carl 1995) have been suggested to involve $\mathrm{K}_{\mathrm{V}} 1$ channel activity. Previous work has also elucidated $\mathrm{K}_{\mathrm{V}}$ current contributions in extrinsic sensory neurons innervating the oesophagus, stomach, ileum and colon (Yoshimura et al. 1996; Glazebrook et al. 2002; Dang et al. 2004). Examining the intrinsic innervation of the gut, Hatton and co-workers reported $\mathrm{K}_{\mathrm{V}} 1.1$ expression in murine, guinea pig and canine myenteric ganglia and ICC (Hatton et al. 2001). However, others describe murine $\mathrm{K}_{\mathrm{V}} 1.1$ expression limited to enteroglial cells, with no expression detected in neurons or ICC (Costagliola et al. 2009). Enteroglial cells and enteric neurons may also express $\mathrm{K}_{\mathrm{V}} 1.2$ 
channel subunits (Costagliola et al. 2009). These points have yet to be resolved. In the human gut we did not find evidence of $\mathrm{K}_{V} 1$ channel protein expression in enteroglial cells or cells resembling ICC. Other currents have been shown to contribute to the activity of these cells, for example ether-a-go-go-related (ERG) K channel and VGCCs in ICC (Huizinga et al. 2004). Our study suggests that $\mathrm{K}_{\mathrm{V}} 1$ channels are widely expressed by enteric neurons of both the myenteric and submucosal plexi and that they have an overlapping yet differential subcellular distribution. This would be expected from heteromultimeric assembly and has been previously shown in the CNS, where $\mathrm{K}_{\mathrm{V}} 1$ channel subunits have overlapping but also unique patterns of subcellular distribution, depending on the region and also neuronal subtype studied. This produces a highly complex arrangement, presumably mirroring functional diversity (Wang et al. 1994; Veh et al. 1995). In contrast to the CNS, $\mathrm{K}_{\mathrm{V}} 1$ subunits in the human ENS appear to be stereotypically directed to subcellular locations and this was consistent between submucosal and myenteric neurons and throughout the gut (with the exception of some subunits in the oesophagus and colon). As the specific expression of $\mathrm{K}_{\mathrm{V}} 1$ subunits in subcellular compartments would help determine the unique firing pattern and thus function of a neuron it is perhaps not surprising that CNS neurons, which are highly diverse, do not exhibit stereotypical patterns of channel expression. Whilst the gut has a large population of neurons (comparable to that of the spinal cord) (Furness 2000), the number of functions and thus complexity is considerably less than that of the CNS. Whilst further studies of $K_{V} 1$ expression by specific neuronal subtypes are required, the stereotypical distribution of $\mathrm{K}_{\mathrm{V}} 1$ channel subunits in the human ENS is at least suggestive of specific functionality.

In canine colonic smooth muscle molecular studies have identified $\mathrm{K}_{\mathrm{V}} 1.2, \mathrm{~K}_{\mathrm{V}} 1.4, \mathrm{~K}_{\mathrm{V}} 1.5$ and $\mathrm{K}_{\mathrm{V}} 1.6$ mRNA (Hart et al. 1993; Epperson et al. 1999). Murine colonic smooth muscle cells express transcripts encoding $K_{V} 1.6$, but lack $K_{V} 1.4$ suggesting there may be inter-species variability (Koh et al. 1999). A single study has previously identified $\mathrm{K}_{\mathrm{V}} 1$ 
channel subunits in the human neuromuscular apparatus. Recordings from isolated human oesophageal smooth muscle cells have revealed a voltage-dependent potassium current and $\mathrm{K}_{\mathrm{V}} 1.2$ and 1.5 mRNA expression in both longitudinal and circular muscle layers were detected (the expression of other $\mathrm{K}_{\mathrm{V}}$ channels was not investigated). Functionally, pharmacological blockade of the muscular voltage-gated potassium current caused an increase in resting tension, suggesting a role in regulation of muscular contraction. However, the agent used in this study (high concentration 4-aminopyridine) non-specifically blocks a number of potassium channel types and so the effect cannot be attributed to $\mathrm{K}_{\mathrm{V}} 1$ channels alone (Wade et al. 1999). Our results demonstrate human GI smooth muscle to be largely devoid of $\mathrm{K}_{\mathrm{V}} 1$ channel expression further suggesting interspecies variability in $\mathrm{GI} \mathrm{K}_{\mathrm{V}} 1$ channel expression. The exception to this and in agreement with the findings of Wade et al. was the finding of $\mathrm{K}_{\mathrm{V}} 1.5$ expression in oesophageal myocytes. The expression of $\mathrm{K}_{\mathrm{V}} 1.5$ in GI smooth muscle was peculiar to the oesophagus. Here, channel expression was mainly, although not exclusively, perinuclear in distribution. In some myocytes, $\mathrm{K}_{\mathrm{V}} 1.5$ was also present in the cell cytoplasm.

The observation that $\mathrm{K}_{\mathrm{V}} 1.5$ is expressed by oesophageal smooth muscle is interesting in relation to our finding of anti-VGKC autoantibodies in patients with Chagas' disease (see Finding 4). Chronic Chagas' disease primarily affects the heart and the gut, specifically regarding the latter, the oesophagus and colon. Why these organs are specifically the focus of disease pathology remains unknown. It is known however, that $\mathrm{K}_{\mathrm{V}} 1.5$ is also expressed by human cardiac muscle (Snyders et al. 1993; Wang et al. 1993), where it is responsible for the ultra-rapid delayed-rectifier current essential for atrial repolarisation (Feng et al. 1997). $\mathrm{K}_{\mathrm{V}} 1.5$ is therefore a channel subunit common to both the heart and the oesophagus. One may tentatively speculate that circulating anti-VGKC autoantibodies, which were present in $14 \%$ of our cohort and are known to impair $\mathrm{K}_{\mathrm{V}} 1$ channel function, may contribute to chagasic oesophageal and heart disease by impairing $\mathrm{K}_{\mathrm{V}} 1.5$ channel function. In support of this hypothesis is the fact that down-regulation of expression of 
this channel has been found to be associated with arrhythmia (Van Wagoner et al. 1997; Lai et al. 1999; Brundel et al. 2001) which is a common feature of chagasic heart disease (Elizari 2002). Due to their function of regulating cellular excitability, impairment of $\mathrm{K}_{\mathrm{V}} 1.5$ oesophageal smooth muscle would be expected to interfere with oesophageal contractility, and this may be relevant to the loss of normal peristalsis and failure of smooth muscle relaxation characteristic of oesophageal achalasia. We did not observe $\mathrm{K}_{\mathrm{V}} 1.5$ immunostaining in colonic smooth muscle. We did however observe $\mathrm{K}_{\mathrm{V}} 1.5$ in colonic submucosal and myenteric neurons. Whilst these findings are interesting and deserve further investigation, the inflammatory fibrotic reaction which damages the cardiac muscle and conduction network and the enteric nervous system remains the most striking feature of this disease. Not all infected individuals, progress to the chronic, symptomatic stage of the disease. About two-thirds of patients remain in an asymptomatic 'indeterminate' phase and no determinants of progression to the symptomatic phase have been identified (Lescure et al. 2010) As discussed in Finding 4, five of the eight patients in which we detected anti-VGKC autoantibodies were asymptomatic. It would be of interest to follow these patients to see if they go on to develop symptomatic disease, as perhaps in some individuals anti-VGKC autoantibodies represent a marker of disease progression, either as a pathological factor, or as a marker of more severe tissue damage which reveals these antigens to the immune system. In either case, the finding would be of significance in guiding a treatment regime which currently lacks definition (Lescure et al. 2010).

Having now demonstrated the presence of $\mathrm{K}_{\mathrm{V}} 1$ channels throughout the human enteric nervous system, the finding of anti-VGKC autoantibodies in patients with primary and secondary GINMD (Knowles et al. 2002; Viallard et al. 2005; Tornblom et al. 2007; Dhamija et al. 2008) takes on new relevance. Whilst not common, the detection of these potentially pathogenic antibodies in patients with GI dysmotility coupled with the knowledge that the human enteric nervous system possesses the antigenic targets could in 
future help guide treatment, particularly in the direction of immunomodulation. The reason for GI involvement in a minority of individuals with anti-VGKC antibodies is unclear, but as discussed in relation to their detection in patients with Chagas' disease (Finding 4), it is likely that an accompanying event such as infection, or neoplasia (Viallard et al. 2005) is necessary. Further investigation of pathogenesis should include a comparative study of enteric $\mathrm{K}_{\mathrm{V}} 1$ channels in disease.

\section{Summary Box: Enteric $K_{V} 1$ channel protein expression}

\section{Previous knowledge}

- $\mathrm{K}_{\mathrm{V}} 1.1$ identified in murine*, guinea pig and canine myenteric neurons and ICC

- $\mathrm{K}_{\mathrm{V}} 1.1$ expressed by murine enteroglia*

- $\mathrm{K}_{\mathrm{V}} 1.2$ expressed by murine myenteric neurons and enteroglia

- $\mathrm{K}_{\mathrm{V}} 1$ subunits expressed by murine $\left(\mathrm{K}_{\mathrm{V}} 1.6\right)$, canine $(1.2,1.4,1.5,1.6)$ and human GI smooth muscle (1.2 and 1.5)

This study

- $\mathrm{K}_{\mathrm{V}}$ 1.1-1.6 expressed in epithelial cells and/or enteric neurons in all gut regions in humans

- Epithelial expression limited to gastric chief cells, surface enterocytes and enteroendocrine cells

- Each subunit exhibits a stereotypical subcellular distribution in enteric neurons

- $\mathrm{K}_{\mathrm{V}} 1.4$ exhibited a unique punctate distribution in gastric and small bowel ganglia

- No expression evident in ICC or enteroglia

- Oesophageal exclusively expresses $\mathrm{K}_{\mathrm{V}} 1$ channels: $\mathrm{K}_{\mathrm{V}} 1.5$

*conflicting results regarding $\mathrm{K}_{\mathrm{V}} 1.1$ distribution in murine myenteric plexus 
Finding 6: $K_{V} 1.4$ is specifically localised to synaptic and axonal locations in enteric ganglia

Of particular interest was the finding of discrete puncta of $\mathrm{K}_{\mathrm{V}} 1.4$ immunoreactivity in ganglia neuropil. $\mathrm{K}_{\mathrm{V}} 1.4$ containing channels are unique amongst $\mathrm{K}_{\mathrm{V}} 1$ channels due to properties of fast-inactivation followed by a slow recovery from inactivation which enable the integration of brief depolarisations over tens of seconds (Pardo et al. 1992; Rasmusson et al. 1995). It is therefore of interest to determine the subcellular distribution of these channels in relation to neuronal functioning. In the central nervous system, particularly the hippocampus, $\mathrm{K}_{\mathrm{V}} 1.4$ has been localised to axons and nerve terminals (Sheng et al. 1993; Cooper et al. 1998). We investigated $K_{V} 1.4$ localisation to enteric synapses and axonal segments using double-labeling immunofluorescent staining for synaptophysin (a synaptic vesicle glycoprotein) and neurofilament (an intermediate filament which is a major component of the axonal cytoskeleton) respectively. In common with hippocampal findings we observed areas of $\mathrm{K}_{\mathrm{V}} 1.4$ and synaptophysin colocalisation. We also saw occasional overlap of $\mathrm{K}_{\mathrm{V}} 1.4$ and neurofilament. Taken together these findings demonstrate that in the human enteric nervous system $\mathrm{K}_{\mathrm{V}} 1.4$ is located at some pre-synatic termini and a subset of axons, consistent with the hippocampal findings (Cooper et al. 1998). The finding of $\mathrm{K}_{\mathrm{V}} 1.4$ immunoreactivity at synaptic termini suggests that $\mathrm{K}_{\mathrm{V}} 1.4$ may contribute to the process of neurotransmitter release (Sheng et al. 1993). Axonal $K_{V} 1$ channels located at or near para-nodal regions are responsible for action potential repolarisation (Hodgkin et al. 1952; Stolinski et al. 1981; Chiu et al. 1982; Wang et al. 1993). As $\mathrm{K}_{\mathrm{V}}$ 1.4-containing channels inactivate quickly and are slow to recover, action potentials propagated along axons bearing $\mathrm{K}_{\mathrm{V}} 1.4$ broaden (Bean 2007). A phenomenon known as activity-dependent spike broadening occurs during high frequency stimulation (Berry 1972; Carew et al. 1977) due to cumulative inactivation of these channels. When this occurs the number of $\mathrm{Ca}^{2+}$ ions entering the pre-synaptic terminal per action potential rises, increasing neurotransmitter release with consequent 
post-synaptic effects (Ma et al. 1995; Geiger et al. 2000; Bischofberger et al. 2002). The finding of this channel in human enteric neurons has potentially important implications regarding the temporal coding of enteric neurotransmission and merits further investigation.

\section{Summary box: Enteric neuronal $K_{V} 1.4$ expression in humans}

Previous knowledge

- $\mathrm{K}_{\mathrm{V}} 1.4$ expression not previously investigated in enteric nervous system

This study

- $\mathrm{K}_{\mathrm{V}} 1.4$ exhibits discrete punctate distribution in enteric ganglia of stomach and small bowel

- Located at synapses and axonal processes

\subsection{CONCLUDING REMARKS}

Despite increased knowledge regarding the pathophysiology of GINMD, the underlying aetiology of these disorders remains largely unknown. Due to phenotypic similarities between GINMD and certain neuromuscular disorders of the periphery which are known to occur as a result of pathogenic autoantibodies against ion channels, and the previous identification of anti-channel autoantibodies in small numbers of patients with GINMD, this thesis investigated ion channel expression in the gut and the presence of anti-channel autoantibodies in idiopathic and infective examples of GI dysfunction. 
The headline findings are:

1. Humoral autoimmunity against currently recognised neuronal antigens is not commonly associated with chronic primary gastrointestinal neuromuscular diseases.

2. Circulating factors present in patients with Chagas' disease modulate colonic smooth muscle contractility through non-cholinergic / non-nitrergic-dependent pathways.

3. Humoral anti-neuronal autoimmunity, particularly in the form of anti-voltagegated potassium channel antibodies, is present in some individuals with Chagas' disease

4. Shaker-related voltage-gated potassium channels are expressed by the human gastrointestinal neuromuscular apparatus

5. $\mathrm{K}_{\mathrm{V}} 1.4$ is located in pre-synaptic termini and axons of a subset of enteric neurons

The results presented in this thesis demonstrate that the human enteric neuromusculature expresses some of the same ion channels that are targets for autoantibodies in the peripheral neuromusculature, but suggest that autoantibodies against these and other channels are not commonly detectable in chronic primary GINMDs. To what extent antichannel antibodies are present in early GINMD remains to be elucidated but is an important question, as if present, early detection and immunomodulatory therapy could in theory prevent or limit deterioration in gut function. Certain phenotypes may increase the likelihood of finding anti-channel antibodies. Dhamija et al., in their investigation of autoimmune gastrointestinal dysmotility conclude: 'Factors that raise the clinical suspicion of AGID were noted in several patients of this report: an antecedent event, subacute onset, evidence of multi-level GI dysmotility, co-existing organ-specific autoimmune disorder (including subtle autonomic/neurological abnormalities, such as postural orthostatism or somatic neuropathy) (Dhamija et al. 2008). A relevant antedecent event could provide an antigenic stimulus. This is clear in paraneoplastic 
cases where a cross-reactive immune response to neural antigens expressed by a tumour leads to neuronal dysfunction. In idiopathic cases it is possible that an occult tumour may have been eliminated by immune surveillance mechanisms that subsequently generate a neurological syndrome (Irani et al. 2010) or even that a tumour may not be detectable until much later: anti-NMDAR encephalitis may precede by years the detection of an ovarian teratoma ((Iizuka et al. 2008). A preceding infection may also provoke an antibody-mediated autoimmune response as demonstrated in patients with Guillain Barré syndrome (Willison et al. 2002). Post-infectious IBS associated with altered cellmediated immunity is now a commonly recognised phenotype (Gwee 2010) and it is interesting that the two patients in whom antibodies (anti-VGKC and $-\alpha 3 \mathrm{nAChR}$ ) were detected in the study of Tornblom et al. had onset of disease after probable acute infectious enteritis. Our finding of anti-VGKC antibodies in patients with Chagas' disease lends further support to the hypothesis that these antibodies can often be a postinfectious phenomenon.

Autoimmune channelopathies are being increasingly identified, a pertinent example being the recent identification of autoantibodies binding aquaporin 4 (a water channel) in patients with neuromyelitis optica (Lennon et al. 2005), a severe inflammatory demyelinating disease of the optic nerves and spinal cord. This discovery has caused a dramatic paradigm shift in the identification and early treatment of this previously poorly understood condition. Further identification of autoimmune channelopathies may open new avenues in the study of gut dysfunction. For now careful selection of GINMD patients in whom to look for autoantibodies based on the conclusions of Dhamija et al. and following the findings of this study, will help to clarify whether or not AGID is a significant aetiological entity. 


\section{Appendices}

\subsection{CONFIRMATORY DOCUMENTS}

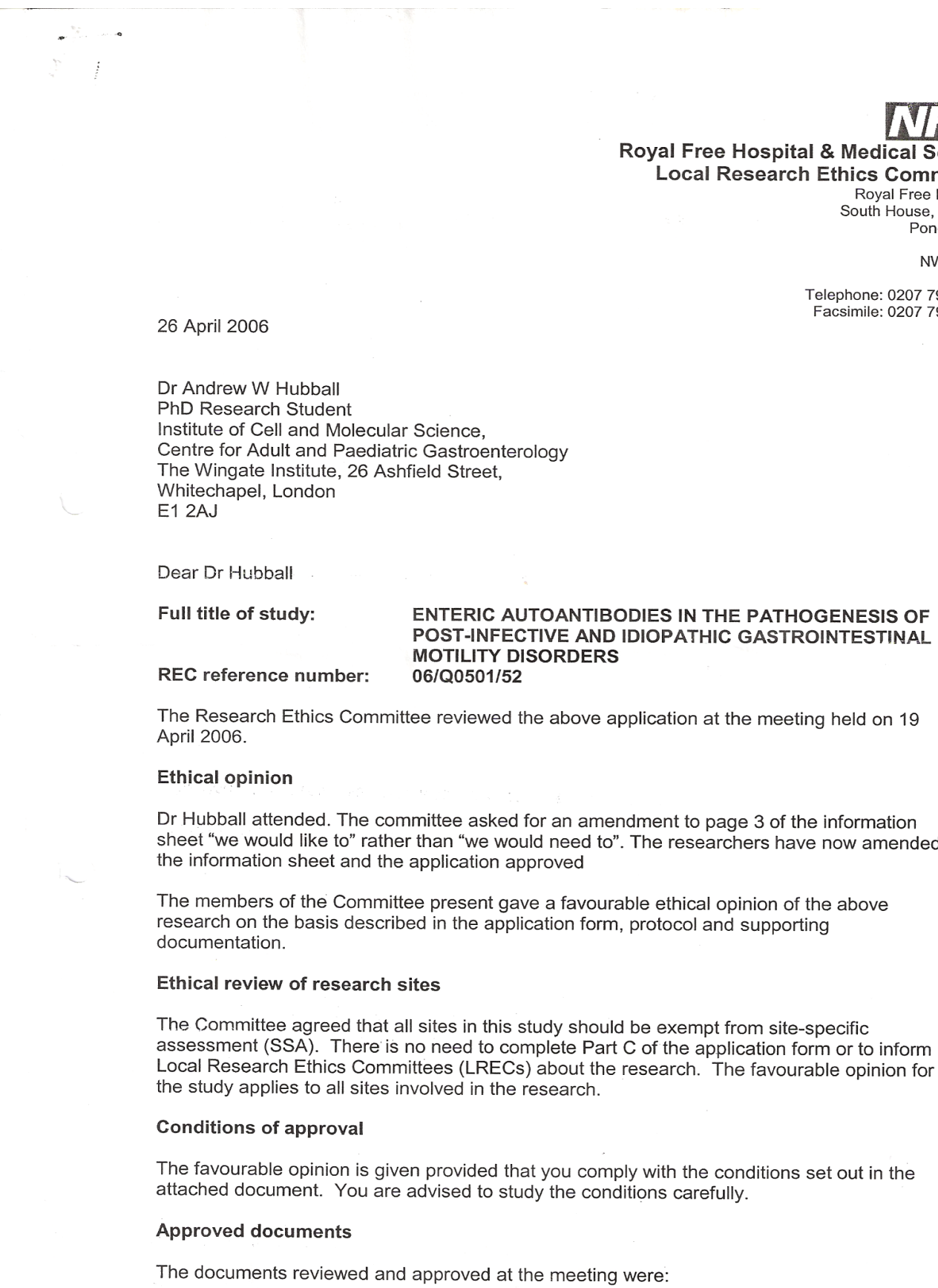

The Research Ethics Committee reviewed the above application at the meeting held on 19 April 2006.

Ethical opinion

Dr Hubball attended. The committee asked for an amendment to page 3 of the information sheet "we would like to" rather than "we would need to". The researchers have now amended the information sheet and the application approved

The members of the Committee present gave a favourable ethical opinion of the above research on the basis described in the application form, protocol and supporting documentation.

Ethical review of research sites

The Committee agreed that all sites in this study should be exempt from site-specific assessment (SSA). There is no need to complete Part $\mathrm{C}$ of the application form or to inform Local Research Ethics Committees (LRECs) about the research. The favourable opinion for the study applies to all sites involved in the research.

\section{Conditions of approval}

The favourable opinion is given provided that you comply with the conditions set out in the attached document. You are advised to study the conditions carefully.

Approved documents

The documents reviewed and approved at the meeting were: 


\begin{tabular}{|l|l|l|}
\hline Document & Version & Date \\
\hline Application & 5.1 & 24 March 2006 \\
\hline Investigator CV & & 20 February 2006 \\
\hline Protocol & 7 & 20 February 2006 \\
\hline Covering Letter & & 24 March 2006 \\
\hline Letter from Sponsor & & 16 February 2006 \\
\hline Peer Review & & \\
\hline Compensation Arrangements & & 16 February 2006 \\
\hline Participant Information Sheet & $1,2,3$ & 20 February 2006 \\
\hline Participant Consent Form & $1.2,3$ & 20 February 2006 \\
\hline Letter from funder & & \\
\hline
\end{tabular}

\section{Research governance approval}

You should arrange for the R\&D Department at all relevant NHS care organisations to be notified that the research will be taking place, and provide a copy of the REC application, the protocol and this letter.

All researchers and research collaborators who will be participating in the research at a NHS site must obtain final research governance approval before commencing any research procedures. Where a substantive contract is not held with the care organisation, it may be necessary for an honorary contract to be issued before approval for the research can be given.

\section{Membership of the Committee}

The members of the Ethics Committee who were present at the meeting are listed on the attached sheet.

\section{Statement of compliance}

The Committee is constituted in accordance with the Governance Arrangements for Research Ethics Committees (July 2001) and complies fully with the Standard Operating Procedures for Research Ethics Committees in the UK.

\section{6/Q0501/52 Please quote this number on all correspondence}

With the Committee's best wishes for the success of this project

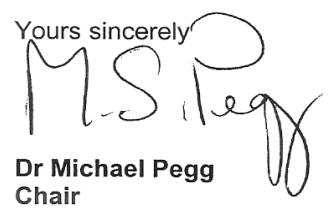

Email: rosemary.brown@royalfree.nhs.uk

Enclosures:

List of names and professions of members who were present at the meeting and those who submitted written comments

Standard approval conditions [SL-AC1 for CTIMPS, SL-AC2 for other studies] 


\section{Royal Free Hospital \& Medical School} Research Ethics Committee Royal Free Hospital South House, Block A Pond Street NW3 2QG

Dr Andrew W Hubball PhD Research Student

Centre for Adult and Paediatric Gastroenterology

The Wingate Institute, 26 Ashfield Street,

Whitechapel, London

E1 2AJ

Dear Dr Hubball

Study title:

ENTERIC AUTOANTIBODIES IN THE PATHOGENESIS OF POST-INFECTIVE AND IDIOPATHIC GASTROINTESTINAL MOTILITY DISORDERS

REC reference: $06 / \mathrm{Q} 050 \cdot 1 / 52$

Amendment number: $\quad$ 06/Q0501/52/AM02

Amendment date:

21 February 2007

The above amendment was reviewed at the meeting of the Sub-Committee of the REC held on 21 March 2007.

Ethical opinion

The members of the Committee present gave a favourable ethical opinion of the amendment on the basis described in the notice of amendment form and supporting documentation.

Approved documents

The documents reviewed and approved at the meeting were:

\begin{tabular}{|l|l|l|}
\hline Document & Version & Date \\
\hline Protocol & 9 & 21 February 2007 \\
\hline Participant Information Sheet & 3.2 & 21 February 2007 \\
\hline Farticipant information Sheet & 2.2 & 2 í Feubruary 2007 \\
\hline Participant Information Sheet & 1.1 & 21 February 2007 \\
\hline Participant Consent Form & 3.2 & 21 February 2007 \\
\hline
\end{tabular}




\begin{tabular}{|l|l|l|}
\hline Participant Consent Form & 2.2 & 21 February 2007 \\
\hline Participant Consent Form & 1.1 & 21 February 2007 \\
\hline $\begin{array}{l}\text { Homerton Hospital R\&D } \\
\text { Approval }\end{array}$ & & 20 February 2007 \\
\hline Amended Protocol Extract & & 21 February 2007 \\
\hline $\begin{array}{l}\text { Amended Patient Information } \\
\text { Sheet Extracts }\end{array}$ & 21 February 2007 \\
\hline $\begin{array}{l}\text { Notice of Substantial } \\
\text { Amendment (non-CTIMPs) }\end{array}$ & 21 February 2007 \\
\hline
\end{tabular}

Membership of the Committee

The members of the Committee who were present at the meeting are listed on the attached sheet.

\section{R\&D approval}

All investigators and research collaborators in the NHS should notify the R\&D office for the relevant NHS care organisation of this amendment and check whether it affects R\&D approval of the research.

\section{Statement of compliance}

The Committee is constituted in accordance with the Governance Arrangements for Research Ethics Committees (July 2001) and complies fully with the Standard Operating Procedures for Research Ethics Committees in the UK.

06/Q0501/52: Please quote this number on all correspondence

Yours sincerely

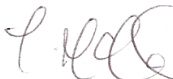

Thomas McQuillan

Committee Co-ordinator

E-mail: Thomas.mcquillan@royalfree.nhs.uk

Enclosures List of names and professions of members who were present at the meeting and those who submitted written comments

Copy to:

Research and Development Dept. Barts and The London NHS Trust I QMUL 


\section{Homerton University Hospital WTS}

NHS Foundation Trust

Research \& Development Committee Chair: Professor Kate Costelo

Kate Costeloe

Director of Research \& Development

Kate.costeloe@homerton.nhs.uk

$\mathrm{Mr}$ Charles Knowles

Department of Surgery

Homerton University Hospital NHS Foundation Trust

Homerton Row

London E9 6SR

Dear Mr Knorviles Clowtes

Re:

Enteric autoantibodies in the pathogenesis of post-infective and idiopathic gastrointestinal motility disorders

REC Ref Number: 06/Q05051/52

Thank you for sending confirmation of your approval from the Research Ethics Committee. I am happy to inform you that the Trust will indemnify against negligent harm that might occur during the course of this project. Should any untoward events occur it is essential that you contact the R\&D Office immediately. If patients or staff are involved in an incident you must also contact the Risk Manager on 02085107649.

Please note that all NHS and social care research is subject to the DoH Research Governance Framework. If you are unfamiliar with the standards contained in this document, you may obtain details from the Trust R\&D Office or from the DoH website (www.dh.gov.uk)

As part of research governance, all investigators accessing individually identifiable personal information are required to comply with current data protection requirements. The Healthcare Records Department will not release patient notes for research purposes without the production of this letter and appropriate identification.

Please inform us if your project is amended and you need to re-submit it to the LREC/MREC or if your project terminates early/requires an extension. This is necessary to ensure that your indemnity cover is valid and also helps the office to maintain up-to-date records for submission to the National Research

Register. Once your study has ended, you will be required to fill out a Project Outcomes form. A copy of any publications arising from the research should be sent to the R\&D Office for use in the R\&D Annual Report. Please be reminded that this hospital should be acknowledged in any publication.

If you believe that protectable intellectual property has arisen from your research, please contact the R\&D Manager who will advise you on the proper course of action.

Please do not hesitate to contact me if you have any further questions.

Yours sincerely

$$
\text { K.h-costule }
$$

Professor Kate Costeloe

Director of Research \& Development

The Homerton The Hospital for Hackney 


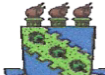 \\ UNIVERSIDADE FEDERAL DO CEARÁ \\ DEPARTAMENTO DE MEDICINA CLÍNICA}

\section{CUSTOMS INVOICE}

Date: 01 DE OUTUBRO DE 2008

HAWB: 608170

Shipper: Universidade Federal do Ceará -HUWC

Prof. Miguel Ângelo Nobre e Souza

Rua: Capital Francisco Pedro, 1290

Bairro: Rodolfo Teófilo

Fone: $55853366.8101-3366.8170$

Fortaleza - CE

Consignee: $\quad$ Mr C.H Knowles BChir PhD FRCS (Gen Surg)

Senior Lecturer and Hon Consultant Colorectal Surgeon,

Centre for Academic Surgery,

3rd Floor Alexandra Wing,

Royal London Hospital,

Whitechapel,

London E1 1BB.

02073777449

07866586766

Contents:

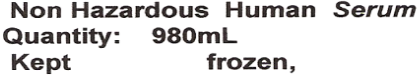

Human material that was neither inoculated with nor exposed to infectious agents of agricultural concern, including zoonotic agents.

Human material containing no animal material and not of tissue culture origin.

Obtained directly from humans, not recombinant, not cultured.

No Commercial Value - For Customs Purposes Only \$10 USD

$\begin{array}{lr}\text { GROSS WEIGHT: } & 16 \mathrm{Kg} \\ \text { NET WEIGHT: } & \mathbf{9 8 0} \\ \text { NUMER OF PIECES: } & -\quad 01\end{array}$

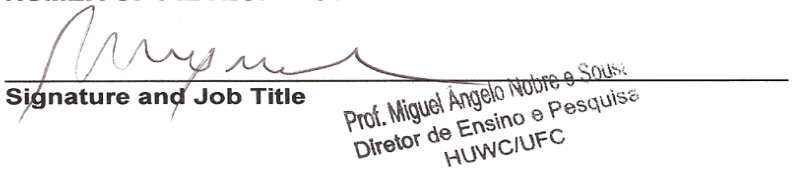




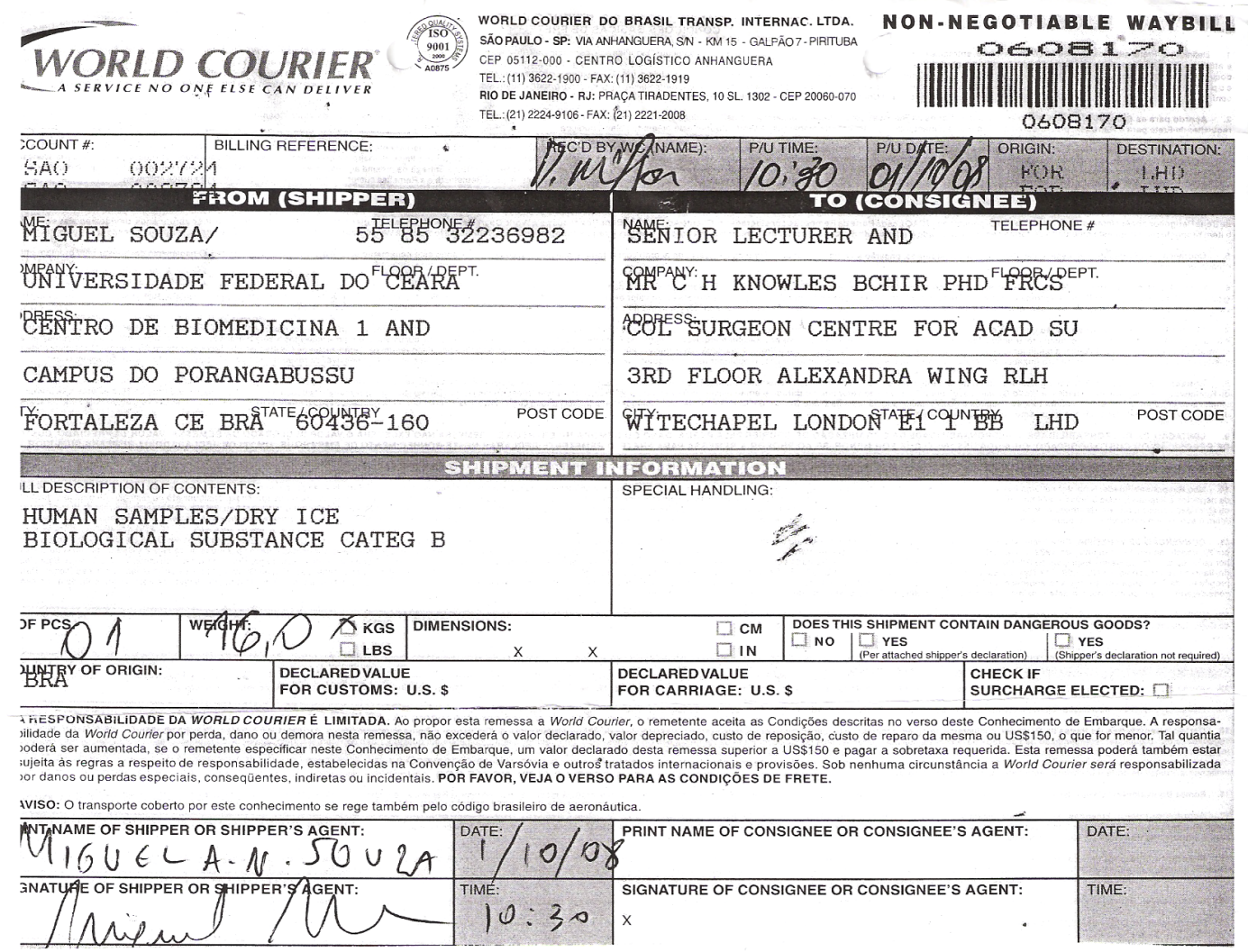

\subsection{ANTI-NEURONAL IMMUNOFLUORESCENT ASSAY}

\section{SOLUTIONS AND REAGENTS}

Solutions and reagents were supplied by Instrumentation Laboratory (UK) Ltd., Warrington, Cheshire, UK (IL), VWR International, Leicestershire, UK (VWR) or Binding Site, Birmingham, UK (Binding Site).

Phosphate buffered saline (PBS) (VWR):

Mounting media (prepared weekly):

$12.5 \mathrm{ml}$ PBS filtered through $0.2 \mathrm{~mm}$ 37.5 glycerol (VWR)

\section{SUBSTRATE}

Monkey cerebellum, 5 well slides (IL) 


\section{INDIRECT-IMMUNOFLUORESCENT STAINING PROTOCOL}

1. Apply $30 \mu 1$ of sera diluted 1:50 to slide well and incubate for $20 \mathrm{~min}$ RT

2. Apply ready-to-use positive controls $(\mathrm{Hu} / \mathrm{Ri}$ and $\mathrm{Yo})$ and incubate as above

3. Rinse off sera with PBS, and soak in PBS for 20 min

4. Dilute FITC-conjugated secondary antibody 1:40 in PBS and microcentrifuge at $13,000 \mathrm{rpm}$ for $5 \mathrm{~min}$.

5. Wipe around sections and apply secondary antibody for 20 min at RT

6. Rinse secondary antibody off with PBS and soak in PBS for 20 min

7. Blot off excess PBS and mount with mounting media

8. Store at $4^{\circ} \mathrm{C}$ until reading

\subsection{RADIOIMMUNOPRECIPITATION ASSAYS}

\section{SOLUTIONS AND REAGENTS}

Solutions and reagents were supplied by Sigma-Aldrich, Poole, Dorset, UK (not stated), Calbiochem-Novabiochem, San Diago, CA, USA (Calbiochem), Binding Site, Birmingham, UK (Binding Site), RSR, Cardiff, UK (RSR), New England Nuclear, Boston, MA, USA (New England) or Perkin Elmer, Waltham, MA, USA (Perkin Elmer)

\subsection{PREPARATION OF CHANNEL EXTRACTS}

Solubilisation buffer (SB), 1 litre: $\quad 25 \mathrm{mM}$ Tris-HCl $\quad 2.15 \mathrm{~g}$

$\left(\mathrm{pH} 7.4\right.$, stored at $-20^{\circ} \mathrm{C}$ until use) $\quad 5 \mathrm{mM}$ HEPES $\quad 1.19 \mathrm{~g}$

$0.32 \mathrm{M}$ Sucrose $\quad 0.32 \mathrm{~g}$

Protease inhibitors:

$\begin{array}{ll}\text { Pepstatin A } & \text { final concentration } 1 \mu \mathrm{M}\end{array}$ 
Leupeptin

Phenylmethylsulphonylflouride (PMSF) final concentration $2 \mu \mathrm{M}$

final concentration $0.1 \mu \mathrm{M}$

Digitonin (50\% pure) (Calbiochem)

2\% Digitonin in solubilisation buffer for VGKC extraction only (DTX):

$20 \mathrm{mM}$ Tris

$100 \mathrm{mM} \mathrm{NaCl}$

$5 \mathrm{mM} \mathrm{KCl}$

Natural $\mathrm{pH} \cong 10.0$, therefore add $\mathrm{HCl}$ to $\mathrm{pH} 7.12$

1. To $2 \mathrm{ml}$ (will be $2.5 \mathrm{ml}$ when $500 \mu \mathrm{l}$ membranes added), add digitonin $0.05 \mathrm{~g}(50 \%)$ to give final concentration of $2 \%$ (active concentration: $1 \%$ ).

2. Incubate in water bath at $37^{\circ} \mathrm{C}$ for $15 \mathrm{~min}$.

3. Just before use, add protease inhibitor: PMSF: $5 \mu$ l of $0.1 \mathrm{M}$ in propan-2-ol (final concentration $0.2 \mathrm{mM}$ ).

\section{Preparation of brain membranes}

1. Remove tissue as soon as possible post-mortem, cut into small pieces and drop into liquid nitrogen. Store at $-70^{\circ} \mathrm{C}$ until use.

2. Take ' $\mathrm{x}$ ' gram of cerebellum, and make up with ice cold SB (with protease inhibitors added immediately before use) to $6-8 \mathrm{x}$ volume of ' $\mathrm{x}$ '.

3. Homogenise using a Polytron Homogeniser (PT10-35, Kinematica GmbH, Littau, Switzerland), full speed 3 x $10 \mathrm{sec}$, resting on ice in-between.

4. Spin $1000 \mathrm{rpm}$ for $10 \mathrm{~min}$

5. Harvest supernatant 
6. Spin $13,000 \mathrm{rpm}$ for $10 \mathrm{~min}$

7. Re-suspend pellet in a volume of SB equivalent to original brain tissue weight

8. Aliquot in $500 \mu \mathrm{l}$ amounts and store at $-70^{\circ} \mathrm{C}$ until use

\section{Voltage-gated potassium channel (VGKC) extract preparation}

(made fresh for immediate use)

$1 \mathrm{M} \mathrm{NaCl}$ in $\mathrm{SB}$ :

$5.84 \mathrm{~g} \mathrm{NaCl}$ in $100 \mathrm{ml} \mathrm{SB}$

1. Defrost aliquots of rabbit brain cortex-membrane preparation.

2. Add stepwise $(2 \times 250 \mu \mathrm{l})$ to $2 \mathrm{ml}$ DTX (with fresh protease inhibitor), mix gently with pipette ( $1: 5$ dilution) (total volume $2.5 \mathrm{ml}$ : see above).

3. Incubate $37^{\circ} \mathrm{C}$ for $15 \mathrm{~min}$.

4. Spin 13,000rpm for 15 min (bench-top Eppendorf centrifuge).

5. Harvest the supernatant (VGKC extract) and store on wet ice until use.

\section{Voltage-gated calcium channel (VGCC) extract preparation}

(made in batches for storage before subsequent use)

4\% digitonin in SB:

Digitonin purchased is $50 \%$ pure. Therefore the solution is made up to $8 \%: 1.6 \mathrm{~g}$ digitonin in $20 \mathrm{ml} \mathrm{SB}$, heated to $60^{\circ} \mathrm{C}$, until dissolved and stored at $4^{\circ} \mathrm{C}$ before use. There is a lot of insoluble material remaining; before use in the assay an aliquot must be centrifuged: 13,000rpm for 5 min and clear supernatant removed for subsequent use.

1. Defrost aliquots of rabbit cerebellum membrane preparation 
2. Add $700 \mu \mathrm{l} \mathrm{SB}$ (with fresh protease inhibitors), vortex, spin 13,000rpm for $5 \mathrm{~min}$ (bench-top Eppendorf centrifuge), discard supernatant.

3. Add $1 \mathrm{ml} \mathrm{SB}$, vortex, spin 13,000rpm for $5 \mathrm{~min}$, discard supernatant.

To the pellet (approx. $200 \mu \mathrm{l}$ ), add: $\quad 100 \mu 1 \mathrm{M} \mathrm{NaCl}$ in $\mathrm{SB}$

$200 \mu 1 \mathrm{SB}$

$500 \mu 14 \%$ digitonin in SB

(therefore the final solution will be $2 \%$ digitonin, $0.1 \mathrm{M} \mathrm{NaCl}$ )

4. Rotate $1 \mathrm{~h}, 4^{\circ} \mathrm{C}$.

5. Spin 13,000rpm for $15 \mathrm{~min}$

6. Remove supernatant, aliquot and store at $-70^{\circ} \mathrm{C}$ as source of VGCCs.

\section{Ganglionic a3 nAChR extract preparation}

1. Grow neuroblastoma cells IMR32 to confluence $\left(5 \% \mathrm{CO}_{2}, 37^{\circ} \mathrm{C}\right)$.

2. Trypsinise cells from three flasks and spin 1000rpm for 5 min

3. Combine the pellets and re-spin 13,000rpm for $5 \mathrm{~min}$

4. Remove the supernatant and freeze at $-80^{\circ} \mathrm{C}$ until use

\section{Detergents}

Triton X100 (t-octylphenoxypolyethoxyethanol)

\section{Radiolabelled toxins and ligands}

The toxins are received as lyophilised powder and are reconstituted with distilled water at a concentration of $0.1 \mu \mathrm{Ci} / 10 \mu \mathrm{l}$, aliquotted into $10 \mu \mathrm{Ci}$ amounts and 
stored $70^{\circ} \mathrm{c}$ until use.

Potassium channels

${ }^{125} \mathrm{I}-\alpha$-dendrotoxin $\left({ }^{125} \mathrm{I}-\alpha-\mathrm{DnTx}\right)$ (Perkin Elmer),

Calcium channels

${ }^{125} \mathrm{I}-\omega$-conotoxin MVIIC $\left({ }^{125} \mathrm{I}-\omega-\mathrm{CmTx}\right), 5 \mu \mathrm{Ci}, \mathrm{S} . \mathrm{A} .>2000 \mathrm{Ci} / \mathrm{mmol}$ (New England)

Ganglionic AChR- $\alpha-3$ channels

${ }^{125}$ I-Epibatidine $\left({ }^{125} \mathrm{I}\right.$-Epi) (Perkin Elmer)

Glutamic acid decarboxylase tracer

${ }^{125}$ I-recombinant $\mathrm{GAD}_{65}$ radioimmunoassay kit (RSR)

\subsection{RADIOIMMUNOPRECIPITATION ASSAYS}

Sheep anti-human IgG (Binding Site)

Washing buffer (PTX): $\quad 0.1 \%$ Triton X100 in 20mM phosphate buffer

$\left(\mathrm{pH} 7.4\right.$, stored at $\left.4^{\circ} \mathrm{C}\right)$

VGKC assay

Radio-labelling using ${ }^{125} I-\alpha$-dendrotoxin $\left({ }^{125} I-\alpha\right.$-DNTX)

1. Defrost $1 \mu \mathrm{Ci}$ of ${ }^{125} \mathrm{I}-\mathrm{DNTX}$ and add $3.5-4 \mathrm{ml}$ of VGKC extract

2. Count on automatic gamma counter (Canberra Packard Cobra II auto-gamma, Meriden ${ }^{\circledR}, \mathrm{CT}$, USA) and adjust to $50,000 \mathrm{cpm} / 50 \mu 1$

3. Incubate at $4^{\circ} \mathrm{C}$ until use 
4. Calculate total amount of labelled extract required $(50 \mu 1 \times$ number of samples), and mix gently with pipette: x $\mu \mathrm{l} \mathrm{VGKC} \mathrm{extract}$ $\mathrm{x} \mu 1 \mathrm{PTX}$ x / 10 125I- $\alpha$-DnTx

5. Transfer to Eppendorfs, and microcentrifuge 13,000rpm for $5 \mathrm{~min}$ before use to remove particulate matter.

\section{Anti-VGKC radioimmunoprecipitation assay}

1. Dilute $10 \mu 1$ patient sera 1:10 with PTX, vortex, spin 13,000rpm for 5 min.

2. Add $50 \mu 1$ of supernatant from each sample of diluted serum to $50 \mu$ l of labelled extract, vortex, and incubate overnight at $4^{\circ} \mathrm{C}$.

3. Add $50 \mu 1$ sheep anti-human IgG to each sample (not microcentrifuged)

4. Incubate $1 \mathrm{~h}$ at room temp.

5. Add $500 \mu 1 \mathrm{PTX}$, vortex, spin at 13,000rpm for $5 \mathrm{~min}$

6. Remove supernatant by suction, wash pellet twice in $500 \mu 1$ PTX.

[N.B if large number of samples, perform steps 12 and 13 stepwise in sets to prevent time dependent dissociation of ${ }^{125}$ I-DNTX-complexes]

7. Remove excess liquid. Count pellet on automatic gamma counter (Canberra Packard Cobra II auto-gamma, Meriden ${ }^{\circledR}$, CT, USA).

\section{VGCC assay}

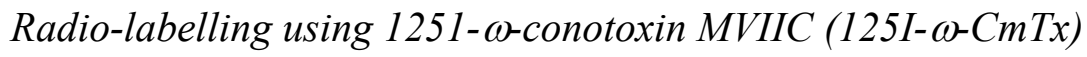

Incubation buffer (IB) $\mathrm{pH} 7.4$, stored at $4^{\circ} \mathrm{C}$ : $\quad 500 \mu$ digitonin $4 \%$

$17.5 \mathrm{ml} \mathrm{dH} 20$

$2 \mathrm{ml} 0.2 \mathrm{M}$ phosphate 
1. Defrost extracts on wet ice

2. In a $7 \mathrm{ml}$ bijou, add $25 \mu 1 \mathrm{IB}$ to $5 \mu 1$ cerebellar extract, i.e. up to a total of $30 \mu 1$ per sample, multiplied by the total number of samples to be tested in assay.

e.g. cerebellar extract for 50 samples $=250 \mu 1$ extract $+1.25 \mathrm{ml} \mathrm{IB}$

3. Defrost $1.0 \mu \mathrm{Ci}$ aliquots of ${ }^{125} \mathrm{I}-\omega$-conotoxin, make up each aliquot with $500 \mu \mathrm{IB}$, spin 13,000rpm for $5 \mathrm{~min}$.

4. Add 5 fmol of ${ }^{125} \mathrm{I}-\omega$-conotoxin per sample, usually 6-10 $\mu 1 /$ sample: see Appendix 3.3 for calculation.

5. Make up total volume / sample to $50 \mu 1$ with IB. i.e. $30 \mu 1+6-10 \mu 1$ (above) +20 $(6-10) \mu 1$. This is the labelled extract preparation.

6. Incubate $2 \mathrm{~h}$ at $4^{\circ} \mathrm{C}$ on wet ice.

\section{Anti-VGCC radioimmunoprecipitation assay}

1. Dilute $10 \mu 1$ test sera diluted 1:10 with IB, vortex, spin 13,000rpm for $5 \mathrm{~min}$.

2. Add $25 \mu 1$ of supernatant from each sample of diluted serum (equivalent to $2.5 \mathrm{ul}$ serum) to $50 \mu 1$ of labelled extract, vortex, and incubate overnight at $4{ }^{\circ} \mathrm{C}$.

3. Add $25 \mu 1$ sheep anti-human IgG to each sample (not microfuged)

4. Incubate $1 \mathrm{~h}$ at $4^{\circ} \mathrm{C}$.

5. Add $1 \mathrm{ml}$ PTX, vortex, spin at 13,000rpm for $5 \mathrm{~min}$

6. Remove supernatant by suction, wash pellet twice in $1 \mathrm{ml}$ PTX.

[N.B if large number of samples, perform steps 12 and 13 stepwise in sets to prevent time dependent dissociation of ${ }^{125} \mathrm{I}-\omega$-conotoxin-complexes].

7. Remove excess liquid. Count pellet on automatic gamma counter (Canberra Packard Cobra II auto-gamma, Meriden ${ }^{\circledR}$, CT, USA).

8. Calculate final binding of labelled extract in pM / 1 using algorithm (Appendix 3.3) 


\section{Ganglionic $\alpha 3-A C h R$ assay}

\section{Radio-labelling using ${ }^{125}$ I-Epibatidine}

1. Defrost IMR32 pellets and wash once in $0.05 \mathrm{M}$ Tris $\mathrm{pH} 7.2$

2. Spin 13,000rpm for $5 \mathrm{~min}$ and remove the supernatant

3. Re-suspend the pellet in $400 \mu 10.05 \mathrm{M}$ Tris $\mathrm{pH} 7.2$ and add $400 \mu 14 \%$ Triton X in $0.05 \mathrm{M}$ Tris $\mathrm{pH} 7.2$ and $10 \mu 1 \mathrm{PMFS}$

4. Vortex the pellet until homogenous and rotate at $4^{\circ} \mathrm{C}$ for $1 \mathrm{~h}$

5. Spin $13,000 \mathrm{rpm}$ for $15 \mathrm{~min}$ and remove the supernatant

6. Determine the amount of extract required by titration by setting out $0,5,10,20$, $30 \mu 1$ made up to $50 \mu 1$ with PTX

7. Defrost $1.0 \mu \mathrm{Ci}$ aliquots of ${ }^{125}$ I-Epibatidine, make up each aliquot with $500 \mu 1$ PTX, spin 13,000rpm for 5 min

8. Aliquot $10 \mu 1$ and count on automatic gamma counter (Canberra Packard Cobra II auto-gamma, Meriden ${ }^{\circledR}, \mathrm{CT}$, USA).

9. Add $50,000 \mathrm{cpm}$ to each titration tube and incubate for $2 \mathrm{~h}$ at $4^{\circ} \mathrm{C}$

\section{Anti- $\alpha 3 n$ AChR radioimmunoprecipitation assay}

1. Dilute $10 \mu 1$ patient sera 1:10 with PTX, vortex, centrifuge $13,000 \mathrm{rpm}$ for $5 \mathrm{~min}$.

2. Add $50 \mu 1$ of supernatant from each sample of diluted serum to $50 \mu 1$ of labelled extract, vortex, and incubate overnight at $4^{\circ} \mathrm{C}$.

3. Add $50 \mu 1$ sheep anti-human IgG to each sample (not microcentrifuged)

4. Incubate $1 \mathrm{~h}$ at room temp.

5. Add $500 \mu 1 \mathrm{PTX}$, vortex, spin at 13,000rpm for $5 \mathrm{~min}$

6. Remove supernatant by suction, wash pellet twice in $500 \mu 1$ PTX.

7. Remove excess liquid. Count pellet on automatic gamma counter (Canberra Packard Cobra II auto-gamma, Meriden $\left.{ }^{\circledR}, \mathrm{CT}, \mathrm{USA}\right)$. 
Glutamic acid decarboxylase assay using ${ }^{125}$ I-GAD 65

1. Dilute $10 \mu \mathrm{l}$ patient sera $1 / 10$ with PTX, vortex, spin 13,000rpm for 5 min.

2. Add $50 \mu \mathrm{l}$ of supernatant from each sample of diluted serum to $50 \mu \mathrm{l}^{125} \mathrm{I}$-labelled human recombinant GAD 65 tracer, vortex, and incubate overnight at $4^{\circ} \mathrm{C}$.

3. Add $50 \mu 1$ sheep anti-human IgG to each sample (not microcentrifuged)

4. Incubate $1 \mathrm{~h}$ at room temp.

5. Add $500 \mu 1 \mathrm{PTX}$, vortex, spin at 13,000rpm for $5 \mathrm{~min}$

6. Remove supernatant by suction, wash pellet twice in $500 \mu 1$ PTX.

[N.B if large number of samples, perform steps 12 and 13 stepwise in sets to prevent time dependent dissociation of complexes of ${ }^{125}$ I-labelled human recombinant GAD 65 tracer ]

7. Remove excess liquid. Count pellet on automatic gamma counter (Canberra Packard Cobra II auto-gamma, Meriden ${ }^{\circledR}$, CT, USA).

\subsection{CONVERSION OF OBSERVED COUNTS TO CONCENTRATIONS}

Final concentrations of antibody are expressed as pmol / litre of serum (i.e. pM).

\section{$V G K C$ and $A C h R$ assays}

Conversion factor $=0.1$ (not isotope date dependent). Therefore final concentration $=$ (observed cpm - mean control cpm) .0.1 


\section{VGCC assays}

Conversion is dependent on isotope decay. The conversion factor $(\kappa)$ may be calculated from a further variable $(\lambda)$ which is date dependent, and taken from the decay table (below), and the volume of serum used ( $2.5 \mu$ for all assays), where:

$\kappa=\lambda(\mathrm{fmol} \mathrm{cpm}) / 2.5 \mu \mathrm{l}$

The final concentrations of antibody are then calculated, where:

$[\mathrm{ab}]=($ observed $\mathrm{cpm}-$ mean control $\mathrm{cpm}) \cdot \kappa$

e.g. on day -15

$\left.\kappa=0.000243(\mathrm{fmol} \mathrm{cpm})^{-1}\right) / 2.5 \mu \mathrm{l}$

$\kappa=0.243\left(\mathrm{pmol} \mathrm{cpm}^{-1}\right) / 2.51$

$\kappa=0.0972\left(\mathrm{pmol} \mathrm{cpm}^{-1} \mathrm{l}^{-1}\right)$

and: $\quad[\mathrm{ab}]=($ observed $\mathrm{cpm}-$ mean control cpm $) .0 .0972$

Total and non-specific binding curves (perform this every 2 months):

Calculate a quantitative precipitation curve for known strongly positive (LEMS) and known negative (healthy control) sera. Total binding is calculated by using increasing serum concentrations in the presence of labelled toxin only, and non-specific binding, by repeating the assay at each serum concentration in the presence of excess unlabelled toxin. The specific binding is calculated at each point by subtracting the non-specific binding from the total binding.

\section{Calculation of volume equivalent of 5 fmol $^{125} \mathrm{I}-\omega$-conotoxin}

Count $10 \mu$ l of diluted ${ }^{125} \mathrm{I}-\omega$-conotoxin $(\mathrm{cpm})$. 
Find "expected count" using decay table (see below) and knowledge of the time period since preparation by the manufacturer. N.B "minus" days are a nuance of the decay curve and manufacture.

Calculate the volume (x) equal to $5 \mathrm{fmol}$ by using the equation:

actual $\mathrm{cpm} / 10 \mu \mathrm{l} \quad=\quad$ expected $\mathrm{cpm} / \mathrm{x}$

i.e. on day -23 , if the observed count for $10 \mu \mathrm{l}$ is $37,000 \mathrm{cpm}$, then:

$37,000 / 10=21,960 / \mathrm{x}$

and $\mathrm{x}=6 \mu \mathrm{l}(1 \mathrm{s.f})$

\section{GAD assay}

The GAD kit contains a calibration kit containing sera with known levels of GAD antibodies (0-300 international units (IU) /ml). All test samples were assessed in an assay containing GAD standards. 


\section{${ }^{125}$ I-w-conotoxin decay table}

\begin{tabular}{|c|c|c|c|c|c|c|c|}
\hline & S.A. $(\mathrm{Ci} / \mathrm{mmol}$ & $1 \mathrm{cpm}-\mathrm{fmol}$ & $5 \mathrm{fmol}-\mathrm{cpm}$ & $\overline{D A Y S}$ & S.A.(Ci/mmol & $1 \mathrm{cpm}-\mathrm{fmol}$ & $5 \mathrm{fmol}-\mathrm{cpm}$ \\
\hline-29 & 2572 & 0.000219 & 22839 & 21 & 1567 & 0.000359 & 13915 \\
\hline-28 & 2556 & 0.000220 & 22697 & 22 & 1548 & 0.000364 & 13746 \\
\hline-27 & 2539 & 0.000222 & 22546 & 23 & 1530 & 0.000368 & 13586 \\
\hline-26 & 2522 & 0.000223 & 22395 & 24 & 1512 & 0.000372 & 13427 \\
\hline-25 & 2505 & 0.000225 & 22244 & 25 & 1495 & $0.000377_{i}$ & 13276 \\
\hline-24 & 2488 & 0.000226 & 22093 & 26 & 1478 & 0.000381 & 13125 \\
\hline-23 & 2473 & 0.000228 & 21960 & 27 & 1461 & 0.000385 & 12974 \\
\hline-22 & 2458 & 0.000229 & 21827 & 28. & 1444 & 0.000390 & 12823 \\
\hline-21 & 2433 & 0.000231 & 21605 & 29 & 1428 & 0.000394 & 12681 \\
\hline-20 & 2414 & 0.000233 & 21436 & 30 & 1412 & 0.000399 & 12539 \\
\hline-19 & 2396 & 0.000235 & 21276 & 31 & 1395 & 0.000404 & 12388 \\
\hline-18 & 2378 & 0.000237 & 21117 & 32 & 1378 & 0.000409 & 12237 \\
\hline-17 & 2359 & 0.000239 & 20948 & 33 & 1362 & 0.000413 & 12095 \\
\hline-16 & 2340 & 0.000241 & 20779 & 34. & 1346 & 0.000418 & 11952 \\
\hline-15 & 2320 & 0.000243 & 20602 & 35 & 1331 & 0.000423 & 11819 \\
\hline-14 & 2300 & 0.000245 & 20424 & 36 & 1316 & 0.000428 & 11686 \\
\hline-13 & 2280 & 0.000247 & 20246 & 37 & 1301 & 0.000433 & 11553 \\
\hline-12 & 2260 & 0.000249 & 20069 & 38 & 1286 & 0.000438 & 11420 \\
\hline-11 & 2240 & 0.000251 & 19891 & 39 & 1271 & 0.000443 & 11286 \\
\hline-10 & 2220 & 0.000254 & 19714 & 40 & 1256 & 0.000448 & 11153 \\
\hline-9 & 2199 & 0.000256 & 19527 & 41 & 1242 & 0.000453 & 11029 \\
\hline-8 & 2178 & 0.000259 & 19341 & 42 & 1228 & 0.004590 & 10905 \\
\hline-7 & 2156 & 0.000261 & 19145 & 43 & 1214 & 0.000464 & 10780 \\
\hline-6 & 2134 & 0.000264 & 18950 & 44 & 1200 & 0.000469 & 10656 \\
\hline-5 & 2112 & 0.000267 & 18755 & 45 & 1186 & 0.000475 & 10532 \\
\hline-4 & 2090 & 0.000269 & 18559 & 46 & 1172 & 0.000480 & 10407 \\
\hline-3 & 2068 & 0.000272 & 18364 & 47 & 1158 & 0.000486 & 10283 \\
\hline--2 & 2046 & 0.000275 & 18168 & 48 & 1144 & 0.000492 & 10159 \\
\hline-1 & 2023 & 0.000278 & 17964 & 49 & 1131 & 0.000498 & 10043 \\
\hline 0 & 2000 & 0.000281 & 17760 & 50 & 1118 & 0.000504 & 9928 \\
\hline 1 & 1977 & 0.000285 & 17556 & 51 & 1105 & 0.000510 & 9812 \\
\hline 2 & 1954 & 0.000288 & 17352 & 52 & 1092 & 0.000516 & 9697 \\
\hline 3 & 1932 & 0.000291 & 17156 & 53 & 1080 & 0.000521 & 9590 \\
\hline 4 & 1910 & 0.000295 & 16961 & 54 & 1068 & 0.000527 & 9484 \\
\hline 5 & 1888 & 0.000298 & 16765 & 55 & 1055 & 0.000534 & 9368 \\
\hline 6 & 1866 & 0.000302 & 16570 & 56 & 1042 & 0.000540 & 9253 \\
\hline 7 & 1844 & 0.000305 & 16375 & 57. & 1030 & 0.000547 & 9146 \\
\hline 8 & 1822 & 0.000309 & 16179 & 58 & 1018 & 0.000553 & 9040 \\
\hline 9 & 1801 & 0.000313 & 15993 & 59 & 1007 & 0.000559 & 8942 \\
\hline 10 & 1780 & 0.000315 & 15806 & 60 & 996 & 0.000565 & 8844 \\
\hline 11 & 1760 & 0.000320 & 15629 & 61 & 984 & 0.000572 & 8738 \\
\hline 12 & 1740 & 0.000324 & 15451 & 62 & 972 & 0.000579 & 8631 \\
\hline 13 & 1720 & 0.000327 & 15274 & 63 & 961 & 0.000586 & 8534 \\
\hline 14 & 1700 & 0.000331 & 15096 & 64 & 950 & 0.000593 & 8436 \\
\hline 15 & 1680 & 0.000335 & 14918 & 65 & 939 & 0.000600 & 8338 \\
\hline 16 & 1660 & 0.000339 & 14741 & 66 & 928 & 0.000607 & 8241 \\
\hline 17 & 1641 & 0.000343 & 14572 & 67 & 918 & 0.000613 & 8152 \\
\hline 18 & 1622 & 0.000347 & 14403 & 68 & 908 & 0.000620 & 8063 \\
\hline 19 & 1604 & 0.000351 & 14244 & 69 & 897 & 0.000628 & 7965 \\
\hline 201 & 1586 & 0.000355 & 14084 & 70 & 886 & 0.000636 & 7868 \\
\hline
\end{tabular}




\subsection{CELL-BASED ASSAY}

\subsection{BACTERIAL CULTURE}

All media components were purchased from Oxoid Ltd., Hampshire, UK

Bacteria: $\quad$ Escherichia coli, strain DH5 $\quad$ a

Lysogeny broth (LB): $10 \mathrm{~g}$ Tryptone

$5 \mathrm{~g}$ Yeasy extract

$5 \mathrm{~g} \mathrm{NaCl}$

$1 \mathrm{~L} \mathrm{dH}_{2} \mathrm{O}$

Transformation buffer-I (TF-I):/L $12 \mathrm{~g} \mathrm{RbCl}$

30ml 1M (pH7.5) K acetate

$1.5 \mathrm{~g}$ calcium chloride dihydrate

150 g glycerol

Make up TF-I to a final volume of $950 \mathrm{ml}$, adjust to $\mathrm{pH} 6.4$ with $0.2 \mathrm{M}$ acetic acid, and divide into $95 \mathrm{ml}$ aliquots. Autoclave, allow to cool, and then add $5 \mathrm{ml}$ of $1 \mathrm{M}$ manganese chloride tetrahydrate.

Transformation buffer-II (TF-II): /L $1.2 \mathrm{~g} \mathrm{RbCl}$

$11 \mathrm{~g}$ calcium chloride dihydrate

$150 \mathrm{~g}$ glycerol

Make up TF-II to a final volume of $980 \mathrm{ml}$ and divide into $98 \mathrm{ml}$ aliquots. Autoclave, allow to cool, and then add $0.5 \mathrm{M} 3$-[N-morpholino]propanesulfonic acid. 


\section{Culture growth and collection}

1. Set up overnight pre-culture(s) by inoculating $2.5 \mathrm{ml} \mathrm{LB}$ in $15 \mathrm{ml}$ test tube

2. In $250 \mathrm{ml}$ conical flask, inoculate $50 \mathrm{ml} \mathrm{LB}$ with $0.5 \mathrm{ml}$ pre-culture

3. Measure optical density (OD) at $650 \mathrm{~nm}$ of culture every hour using a Unicam Helios $\beta$ UV spectrophotometer (Unicam, Cambridge, UK)

4. Plot log OD650nm vs. time

5. When OD is predicted to rise above 0.1 , remove $7 \mathrm{ml}$ and place in ice cold bottle

6. Measure OD of $1 \mathrm{ml}$ and transfer exactly $0.5 \mathrm{OD}$ units (0.5/OD observed) into fresh chilled tube

7. Centrifuge $13,000 \mathrm{rpm}, 4^{\circ} \mathrm{C}$ for $5 \mathrm{~min}$ and remove supernatant by aspiration

8. Continue to collect $0.5 \mathrm{OD}$ units every hour until growth is complete

$\mathrm{OD}=0.5-1.0 \quad$ remove $2.5 \mathrm{ml}$, use $1 \mathrm{ml}$ for OD measurement and rest to take sample

1.0-2.0 remove $1 \mathrm{ml}$ and mix with $1 \mathrm{ml}$ ice cold saline, use $0.9 \mathrm{ml}$ for OD measurement and rest to take sample

2.0-3.0 remove $1 \mathrm{ml}$ and mix with $2 \mathrm{ml}$ ice cold saline, use $1 \mathrm{ml}$ for OD measurement and rest to take sample

3.0-4.0 remove $1 \mathrm{ml}$ and mix with $3 \mathrm{ml}$ ice cold saline, use $1 \mathrm{ml}$ for OD measurement and rest to take sample

\section{Preparation of competent cells}

Use sterile technique throughout

1. Inoculate $2.5 \mathrm{ml}$ of $\mathrm{LB}$ and grow overnight at $37^{\circ} \mathrm{C}$ with moderate agitation $(250$ rpm) 
2. Inoculate $50 \mathrm{ml} \mathrm{LB}$ with $0.5 \mathrm{ml}$ of overnight culture

3. Grow at $37^{\circ} \mathrm{C}, 250 \mathrm{rpm}$ until OD is $0.4-0.5$

4. Centrifuge $4000 \mathrm{rpm}$ for $15 \mathrm{~min}$ at $4^{\circ} \mathrm{C}$

5. Remove supernatant and re-suspend in $4 \mathrm{ml}$ of TF-I

6. Place on ice for $15 \mathrm{~min}$

7. Centrifuge $2,500 \mathrm{rpm}$ for $15 \mathrm{~min}$ at $4^{\circ} \mathrm{C}$

8. Resuspend in $2 \mathrm{ml} \mathrm{TF-2}$

9. Keep cells on ice until use, or aliquot into $0.2 \mathrm{ml}$ and store at $-80^{\circ} \mathrm{C}$

\subsection{BACTERIAL TRANSFORMATION}

Plasmid: cDNA encoding full length muscarinic 2 human cholinergic receptor (GenBank NM_001006630) cloned in- frame using the restriction sites EcoRI-XhoI in the polylinker region of pcDNA3 (Invitrogen Ltd., Paisley, UK). Provided by Dr Carl Nelson, University of Leicester, UK.

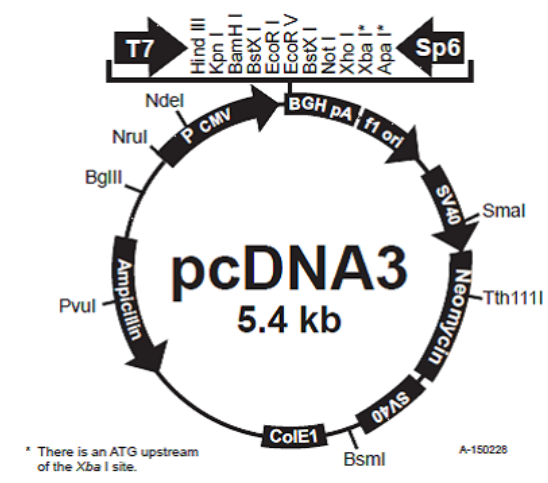

1. Add various dilutions of plasmid DNA (1-50 $\mathrm{ng}$ in $4 \mu \mathrm{l})$ to $0.2 \mathrm{ml}$ aliquots of competent cells

2. Mix gently and incubate on ice for $40 \mathrm{~min}$

3. Heat shock at $42^{\circ} \mathrm{C}$ for $1 \mathrm{~min}$

4. Place on ice for $5 \mathrm{~min}$ 
5. Add $0.7 \mathrm{ml}$ of pre-warmed LB and incubate at $37^{\circ} \mathrm{C}$ for $30-60 \mathrm{~min}$

6. Centrifuge $13,000 \mathrm{rpm}$ for $5 \mathrm{~min}$ and remove $0.7 \mathrm{ml}$ of supernatant

7. Resuspend cells in remainder of supernatant and plate out onto plates containing L agar and ampicillin

8. Allow to dry, cover and incubate overnight at $37^{\circ} \mathrm{C}$

\subsection{DNA ISOLATION AND SEQUENCING}

\section{DNA purification}

Miniprep: for plasmid yields of less than $10 \mu \mathrm{g}$

QIAprep ${ }^{\circledR}$ Spin Miniprep kit (Qiagen Ltd., West Sussex, UK)

1. Centrifuge overnight cultre at $4,000 \mathrm{rpm}, 4{ }^{\circ} \mathrm{C}$ for $5 \mathrm{~min}$

2. Re-suspend pelleted cells in $250 \mu$ Buffer P1 (in kit) and transfer to a microcentrifuge tube

3. Add $250 \mu 1$ Buffer P2 (in kit) and gently invert the tube 4-6 times to mix

4. continue inverting the tube until the solution becomes viscous and slightly clear. Do not allow the lysis reaction to proceed for more than $5 \mathrm{~min}$

5. Add $350 \mu \mathrm{l}$ Buffer N3 (in kit) and invert the tube immediately but gently 4-6 times

6. Centrifuge for $10 \mathrm{~min}$ at $13,000 \mathrm{rpm}$

7. Remove supernatant and apply to the QIAprep spin column (in kit) by decanting or pipetting

8. Centrifuge for 30-60 s. Discard the flow-through

9. (Optional): Wash the QIAprep spin column by adding $0.5 \mathrm{ml}$ Buffer PB (in kit) and centrifuging for 30-60 s. Discard the flow-through. 
10. Wash QIAprep spin column by adding $0.75 \mathrm{ml}$ Buffer PE and centrifuging for $30-60 \mathrm{~s}$

Maxiprep: for plasmid yields greater than $10 \mu \mathrm{g}$

Purelink $^{\mathrm{TM}}$ HiPure Plasmid (Maxi) Filter Purification Kit (Invitrogen Ltd., Paisley, UK)

1. Place the Maxi Column on the PureLink ${ }^{\mathrm{TM}}$ Nucleic Acid Purification Rack (in kit)

2. Apply $30 \mathrm{~mL}$ EQ1 buffer (in kit) directly into the Filtration Cartridge, which is inserted into the Maxi Column

3. Allow the solution in the HiPure Filter Maxi Column to drain by gravity flow

4. Prepare the cell lysate (see below) while the HiPure Filter Maxi Column is equilibrating.

5. For high copy number plasmids, use $100-200 \mathrm{~mL}$ of an overnight LB culture per sample.

6. For low copy number plasmids, harvest $250-500 \mathrm{~mL}$ of an overnight LB culture per sample.

7. Harvest the cells by centrifuging the overnight LB culture at 4,000 rpm for 10 minutes. Remove supernatant

8. Add $10 \mathrm{~mL} \mathrm{R3}$ buffer (in kit) with RNase A to the pellet and resuspend the cells until homogeneous

9. Add $10 \mathrm{~mL} \mathrm{L7}$ buffer (in kit) and mix gently by inverting the capped tube until the lysate mixture is thoroughly homogeneous. Do not vortex.

10. Incubate at room temperature for 5 minutes

11. Add $10 \mathrm{~mL} \mathrm{~N} 3$ buffer (in kit) and mix immediately by inverting the tube until the mixture is thoroughly homogeneous. Do not vortex.

12. Transfer the precipitated lysate including all the precipitated material into the equilibrated HiPure Filter Maxi Column 
13. Let the lysate run through the filter by gravity flow until the flow stops $(10-15$ minutes) or becomes very slow ( $<1$ drop per 10 seconds)

14. Discard the flow through

15. Optional: The final DNA yield may be increased by washing the residual bacterial lysate in the HiPure Filter Maxi Column with $10 \mathrm{~mL}$ W8 buffer (in kit).

16. Let the buffer flow through the HiPure Filter Maxi Column by gravity flow until the flow stops or dripping becomes very slow

17. Immediately after the HiPure Filter Maxi Column has stopped dripping, remove the inner Filtration Cartridge from the column and discard.

18. Wash the Maxi column with $50 \mathrm{~mL}$ of W8 buffer. Allow the solution in the column to drain by gravity flow and discard the flow-through.

\section{Eluting DNA}

1. Place a sterile $50-\mathrm{mL}$ centrifuge tube (elution tube) under the HiPure Filter Maxi column.

2. Add $15 \mathrm{~mL}$ E4 buffer (in kit) to the Maxi column and allow the solution to drain by gravity

3. Discard the HiPure Filter Maxi column.

\section{DNA precipitation}

1. Add $10.5 \mathrm{~mL}$ isopropanol to the DNA to the elution tube and mix well.

2. Centrifuge the tube at $10,000 \mathrm{rpm}$ for $30 \mathrm{~min}$ at $4{ }^{\circ} \mathrm{C}$

3. Remove and discard the supernatant

4. Add $5 \mathrm{~mL} 70 \%$ ethanol to resuspend the DNA pellet.

5. Centrifuge the tube at $10,000 \mathrm{rpm}$ for $5 \mathrm{~min}$ at $4^{\circ} \mathrm{C}$

6. Remove and discard the supernatant.

7. Air-dry the pellet for $10 \mathrm{~min}$

8. Resuspend the DNA pellet in $500 \mu \mathrm{L}$ TE Buffer (in kit) 
9. For low copy number plasmids, use $200 \mu \mathrm{L}$ TE

10. Transfer the supernatant (DNA sample) into a fresh tube.

11. Store the purified DNA at $-20{ }^{\circ} \mathrm{C}$, or store at $4{ }^{\circ} \mathrm{C}$ and proceed to desired downstream application.

\section{DNA digestion}

Restrictions enzymes (New England BioLabs UK Ltd., Hertfordshire, UK):

EcoR1. Recognition site: $\quad 5$ '...G ${ }^{\S}$ AATTC ...3'

$$
\text { 3'.... } \text { TTAA }^{\sqrt{3}} \mathrm{G} \ldots .5 \text {, }
$$

NEBuffer EcoR1 (supplied with enzyme):

$100 \mathrm{mM}$ Tris- $\mathrm{HCl}$

$50 \mathrm{mM} \mathrm{NaCl}$

$10 \mathrm{mM} \mathrm{MgCl} 2$

$0.025 \%$ Triton X-100

Reaction conditions:

Incubate 5 units of enzyme per $\mu \mathrm{g}$ of DNA for $1 \mathrm{~h}$ at $37^{\circ} \mathrm{C}$

Xho1. Recognition site: $\quad$ 5'.... ${ }^{\natural}$ TCGAG...3'

$$
\text { 3'...GAGCT }{ }^{\natural} \mathrm{C} \ldots 5^{\prime}
$$

NEBuffer 4 (suuplied with enzyme):

$20 \mathrm{mM}$ Tris-acetate

$50 \mathrm{mM}$ potassium acetate

$10 \mathrm{mM}$ magnesium acetate

$1 \mathrm{mM}$ dithiothreitol 
Reaction conditions:

Incubate 5 units of enzyme supplemented with $100 \mu \mathrm{g} / \mathrm{ml}$ bovine serum albumin for $1 \mathrm{~h}$ at $37^{\circ} \mathrm{C}$

DNA sequencing

Agarose gel electrophoresis

5x TBE buffer: $\quad 54 \mathrm{~g}$ Tris

$27.5 \mathrm{~g}$ Borate (orthoboric acid)

$20 \mathrm{ml} 0.5 \mathrm{M}$ EDTA (pH8)

$980 \mathrm{ml} \mathrm{dH_{2 } \mathrm { O }}$

Mix Tris, Borate and $\mathrm{dH}_{2} \mathrm{O}$, then add EDTA. Make to final volume of $1 \mathrm{~L}$.

0.5 M EDTA (pH8): 18.6 g EDTA

$175 \mathrm{ml} \mathrm{dH} \mathrm{H}_{2} \mathrm{O}$

$2 \mathrm{~g} \mathrm{NaOH}$

Increase final volume to $200 \mathrm{ml}$, check $\mathrm{pH}$ and autoclave.

1\% agarose gel: $\quad 1 \mathrm{~g}$ agarose

$10 \mathrm{ml} 5 \mathrm{x}$ TBE

$90 \mathrm{ml} \mathrm{H}_{2} \mathrm{O}$

Melt in microwave, cool under tap and add ethidium bromide to final concentration of 25 $\mu \mathrm{g} / \mathrm{ml}$.

6x gel loading buffer: $2.5 \mathrm{ml}$ bromophenol blue

$2.5 \mathrm{ml}$ xylene cyanol FF

$4.0 \mathrm{ml}$ Ficoll in $\mathrm{H}_{2} \mathrm{O}$

Store at room temperature 


\subsection{MAMMALIAN CELL CULTURE}

Tissue culture plastic-wear, all from Appleton Woods Ltd. Birmingham, UK:

$75 \mathrm{~cm}^{2}$ Corning tissue culture flasks

6 and 24-well plates

\section{Media and solutions:}

Dulbecco's Modified Eagles Medium (DMEM) containing 4500 mg/l glucose, Lglutamine and sodium pyruvate (Sigma-Aldrich, Poole, Dorset, UK)

Antibiotic - antimycotic solution, containing / $\mathrm{ml}$, in 0.85 saline: 10,000 units of penicillin, 10,000 $\mu \mathrm{g}$ streptomycin, $25 \mu \mathrm{g}$ amphotericin (Invitrogen Ltd., Paisley, UK)

DMEM+ab: DMEM containing antibiotic - antimycotic solution

DMEM wash: DMEM containing 4.6 g/l HEPES

DMEM diluent: DMEM containing $4.6 \mathrm{~g} / 1$ HEPES and $1 \%$ BSA

Poly-L lysine (Sigma-Aldrich)

Poly-L lysine solution: $\quad 1 \mathrm{ml}$ poly-lysine

$100 \mathrm{ml}$ PBS

Trypsin-EDTA solution (Invitrogen) 


\section{Harvesting and counting of HEK cells}

1. Aspirate off medium

2. Wash cells with $5 \mathrm{ml} \mathrm{PBS}$ and aspirate off

3. Detach cells by adding $1-2 \mathrm{ml}$ of trypsin and incubating at $37^{\circ} \mathrm{C}$ for $1 \mathrm{~min}$

4. Resuspend cells in $10 \mathrm{ml}$ of DMEM $+\mathrm{ab}$

5. Transfer to $50 \mathrm{ml}$ Universal tube and take off $10 \mu 1$ to count using a haemocytometer

6. Centrifuge for $6 \mathrm{~min}$ at $1000 \mathrm{rpm}$

\section{Seeding of HEK cells}

1. In a flow cabinet, place 3 coverslips / well into a six well tray

2. Add $2 \mathrm{ml}$ of poly-L lysine solution into each well

3. Leave for $20 \mathrm{~min}$

4. Aspirate off poly-L lysine solution and allow to dry for $2 \mathrm{~h}$

5. Make a $12 \mathrm{ml}$ cell suspension with DMEM+ab+FCS containing $1.8 \times 10^{6}$ cells

6. Add $2 \mathrm{ml}$ of cell suspension to each well and incubate overnight

7. Transfect the following day

\section{HEK cell transfection}

1. Make up transfection solution according to number of wells:

Mix in two separate tubes (per well):

$4 \mu \mathrm{g}$ DNA

250 - (x $\mu 1$ DNA) ab-free DMEM: incubate for $5 \mathrm{~min}$ 
$10 \mu 1$ lipofectamine

$240 \mu \mathrm{ab}$-free DMEM

2. Mix the contents of the two tubes and incubate for $15 \mathrm{~min}$ RT

3. Change medium of wells to ab-free DMEM ( $2 \mathrm{ml} /$ well) and add $500 \mu \mathrm{l}$ of transfection solution to each well

4. Incubate overnight at $37^{\circ} \mathrm{C}$

5. Change medium to ab+DMEM

6. Incubate overnight at $37^{\circ} \mathrm{C}$

7. The following day cells are ready for immunostaining

\section{Immunofluorescent staining of transfected HEK cells}

1. Transfer coverslips to a mew 24 - well plate containing / well $250 \mu 1 \mathrm{PBS}$

2. Wash with PBS x 2 aspirating each time

3. Fix cells with $4 \%$ formaldehyde for $10 \mathrm{~min}$

4. Aspirate off formaldehyde and wash with PBS x 3

5. Permeabilise cells to be stained with anti-M2 antibody by adding $250 \mu 1$ Triton $\mathrm{X}$ for $10 \mathrm{~min}$

6. Aspirate off Triton $\mathrm{X}$ and wash with DMEM wash $\mathrm{x} 3$

7. Dilute each serum sample, 1:20 in DMEM diluent

8. Dilute anti- $\mathrm{M}_{2}$ primary antibody 1:200 in DMEM diluent

9. Add $250 \mu \mathrm{l}$ of serum or anti-M $\mathrm{M}_{2}$ antibody solution / per well

10. Incubate for $1 \mathrm{~h}$ at $37^{\circ} \mathrm{C}$

11. Remove by aspiration

12. Wash coverslips with $250 \mu 1$ DMEM wash $\mathrm{x} 2$, then PBS $\mathrm{x}$, pipetting and aspirating each time

13. Aspirate off formaldehyde and wash 3 times with PBS 
14. Permeabilise cells

15. Add $250 \mu \mathrm{l}$ of secondary, fluorescently conjugated anti-human antibody (Diluted 1:700 in DMEM diluent) to each well and incubate for 45 min in the dark at RT

16. Aspirate off secondary antibody and wash with DMEM wash x 3 and PBS x 2

17. Transfer coverslips to microscope slides, mounting $3-4$ per slide using fluorescent mounting medium containing DAPI, 1:1000

18. Store at $4{ }^{\circ} \mathrm{C}$ before reading

\subsection{IMMUNOHISTOCHEMISTRY}

\subsection{SOLUTIONS AND REAGENTS}

All solution and reagents were supplied by VWR International, Lutterworth, Leicestershire, UK (not stated), Dako, Ely, Cambridgeshire, UK (Dako), Bio-Genex Laboratories, San Ramon, CA, USA (Bio-Genex), or Sigma Aldrich, Poole, Dorset, UK (Sigma). R.T.U Vectastain Universal Elite ABC kit was supplied by Vector Laboratories Inc., Burlingame, CA, USA (Vector) (Cat. No. PK-7200).

$\mathrm{IMS}=100 \%$ industrial methylated spirits.

Endogenous peroxidase blocking $\quad 6 \mathrm{ml} \mathrm{H}_{2} \mathrm{O}_{2}$

solution

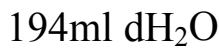

Acid Alcohol

$300 \mathrm{ml} \mathrm{dH} \mathrm{H}_{2} \mathrm{O}$

$700 \mathrm{ml}$ IMS

$10 \mathrm{ml}$ conc. $\mathrm{HCl}$

(added to $990 \mathrm{ml}$ of the above)

Gill's Haematoxylin

$1 \mathrm{~L} \mathrm{dH_{2 }} \mathrm{O}$

$625 \mathrm{ml}$ ethylene glycol, 


\begin{tabular}{|c|c|}
\hline & $10 \mathrm{~g}$ haematoxylin \\
\hline & $1 \mathrm{~g}$ sodium iodate \\
\hline & $176 \mathrm{~g}$ aluminium sulphate \\
\hline & $50 \mathrm{ml}$ glacial acetic acid \\
\hline & 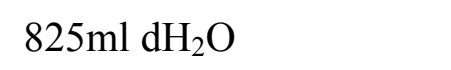 \\
\hline $1 \%$ Eosin & $1 \mathrm{~g}$ eosin $\mathrm{Y}$ \\
\hline
\end{tabular}

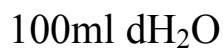

Immunohistochemistry

Endogenous peroxidase $\quad 3 \mathrm{ml} 30 \% \mathrm{H}_{2} \mathrm{O}_{2}$

block (3\%) $\quad 97 \mathrm{ml} \mathrm{100 \%} \mathrm{methanol}$

REAL ${ }^{\text {TM }}$ Antibody diluent (DAKO)

Wash buffer (DAKO)

$0.05 \mathrm{~mol} / \mathrm{L}$ Tris $\mathrm{HCl}$

$0.15 \mathrm{~mol} / \mathrm{L} \mathrm{NaCl}$

$0.05 \%$ Tween 20

Vectastain $\mathrm{ABC}$ Elite reagent (Vector)

$\begin{array}{ll}\text { Diaminobenzidine } & 1 \mathrm{ml} \text { substrate buffer } \\ \text { tetrachloride (DAB) solution } & 1 \text { drop DAB chromagen } \\ \text { (Bio-Genex) } & \end{array}$

Phosphate buffered saline (PBS)

Mounting medium for immunohistochemistry: Canada balsam 
Fluorescence mounting medium (Dako)

\section{Antigen retrieval}

Citrate buffer solution(Vector) Citrate buffer stock 100ml

$\mathrm{dH}_{2} \mathrm{O} 900 \mathrm{ml}$

\subsection{FIXATION AND PROCESSING OF TISSUE}

1. Fix tissue for at least $24 \mathrm{~h}$ in formal saline and embed in paraffin wax.

2. Cut $3 \mu \mathrm{m}$ thick sections and float in a warm water bath

3. Pick up sections on negatively charged microscope slides (VWR International, Leicestershire, UK) and allow water to drain off

4. Dry sections overnight at $37^{\circ} \mathrm{C}$

\subsection{HAEMATOXLYIN AND EOSIN STAINING}

1. Dewax sections in xylene $(2 \times 2 \mathrm{~min})$

2. Remove xylene with IMS (2x 2 min)

3. Wash in running tap water $5 \mathrm{~min}$

4. Stain in Gill's haematoxylin for $20 \mathrm{~min}$

5. Wash in running tap water $5 \mathrm{~min}$

6. Differentiate by dipping in $1 \%$ acid alcohol

7. Wash in running tap water 5 min

8. Stain in eosin for $3 \mathrm{~min}$

9. Dip in tap water and blot

10. Dehydrate rapidly in IMS (2x $2 \mathrm{~min})$

11. Clear in xylene $(2 \times 2 \mathrm{~min})$ 
12. Mount in Canada balsam

\subsection{INDIRECT-IMMUNOHISTOCHEMICAL STAINING PROTOCOL}

1. Dewax sections in xylene $(2 \times 2 \mathrm{~min})$

2. Remove xylene with IMS (2x $2 \mathrm{~min})$

3. Wash in running tap water 5 min

4. Antigen retrieval (see below)

5. Wash in running tap water 5 min

6. Incubate sections in endogenous peroxidase blocking solution for $15 \mathrm{~min}$

7. Wash in running tap water for $5 \mathrm{~min}$

8. Soak in wash buffer (WB) $2 \mathrm{~min}$

9. Dry carefully around sections and encircle with ImmEdge pen (Vector Labs)

10. Apply blocking serum for $20 \mathrm{~min}$ : $25 \%$ normal horse serum (in kit)

11. Tip off blocking serum.

12. Apply primary antibody at the appropriate dilution in antibody diluent for $60 \mathrm{~min}$ RT (antibody diluent only to negative control sections)

13. Rinse sections with WB and soak in WB 2x 5 min

14. Wipe around sections and apply biotinylated secondary antibody diluted 1:250 in wash buffer for $30 \mathrm{~min}$ at RT

15. Apply Vectastain Elite ABC reagent (from kit) for 30 min RT

16. Rinse sections with WB and soak in WB 2x 4 min

17. Make up DAB solution and apply for 5 min (examine positive control slide for progress of development)

18. Wash in running tap water for $5 \mathrm{~min}$

19. Counterstain in Gill's Haematoxylin for $2 \mathrm{~min}$

20. Wash in running tap water for 5 min 
21. Differentiate by dipping two to three times in $1 \%$ acid alcohol

22. Wash in running tap water for $5 \mathrm{~min}$

23. Dehydrate in IMS

24. Clear in xylene

25. Mount in Canada balsam

\subsection{INDIRECT-IMMUNOFLUORESCENT STAINING PROTOCOL}

1. Dewax sections in xylene $(2 \times 2 \mathrm{~min})$

2. Rehydrate with IMS (2x $2 \mathrm{~min})$

3. Wash in running tap water 5 min

4. Antigen retrieval (see below)

5. Wash in running tap water $5 \mathrm{~min}$

6. Soak in PBS $2 \times 5$ min

7. Dry carefully around sections and encircle with ImmEdge pen (Vector Labs)

8. Apply serum-free protein-block for $10 \mathrm{~min}$ :

9. Tip off blocking serum.

10. Apply both primary antibodies in single solution at the appropriate dilution in PBS for 60 min RT (antibody PBS only to negative control sections)

11. Soak slides in PBS 2x 5 min

12. Wipe around sections and apply Alexa-fluor ${ }^{\mathrm{TM}}$ secondary antibodies diluted in PBS (1:200), in a single dilution (finally 1:100) in PBS for 45 min at RT

13. Soak slides in PBS 2x 5 min

14. Mount in fluorescence mounting medium containing DAPI diluted 1:1000

15. Store at $4^{\circ} \mathrm{C}$ until viewing 


\section{Antigen retrieval}

1. Place sections into plastic staining rack leaving a gap between the slides

2. Place rack into a large glass staining trough containing exactly $1000 \mathrm{ml}$ of the antigen retrieval buffer

3. Cover the trough with cling film and make several holes in the cling film

4. Place the trough in the microwave and run at full power for 25 minutes

5. With care, remove trough from microwave

6. Carefully remove cling film

7. Leave sections to stand in the hot buffer for 5 minutes

8. Return to original technique 


\section{REFERENCES}

Adams, R. D. and M. Victor (1993). Myasthenia Gravis and Episodic Forms of Muscular Weakness. Principles of Neurology. W. J. Lamsback and M. Navrozov. New York, McGraw-Hill: 1252-1270.

Aggestrup, S., R. Uddman, F. Sundler, J. Fahrenkrug, R. Hakanson, H. R. Sorensen and G. Hambraeus (1983). Lack of vasoactive intestinal polypeptide nerves in esophageal achalasia. Gastroenterology 84(5 Pt 1): 924-7.

Ahmed, S. S., F. K. Tan, F. C. Arnett, L. Jin and Y. J. Geng (2006). Induction of apoptosis and fibrillin 1 expression in human dermal endothelial cells by scleroderma sera containing anti-endothelial cell antibodies. Arthritis Rheum 54(7): 2250-62.

Akesson, A., F. A. Wollheim, H. Thysell, T. Gustafson, L. Forsberg, O. Pahlm, P. Wollmer and B. Akesson (1988). Visceral improvement following combined plasmapheresis and immunosuppressive drug therapy in progressive systemic sclerosis. Scand J Rheumatol 17(5): 313-23.

Almon, R. R., C. G. Andrew and S. H. Appel (1974). Serum globulin in myasthenia gravis: inhibition of alpha-bungarotoxin binding to acetylcholine receptors. Science 186(4158): 55-7.

Altermatt, H. J., M. Rodriguez, B. W. Scheithauer and V. A. Lennon (1991). Paraneoplastic anti-Purkinje and type I anti-neuronal nuclear autoantibodies bind selectively to central, peripheral, and autonomic nervous system cells. Lab Invest 65(4): 412-20.

Apiwattanakul, M., A. McKeon, S. J. Pittock, T. J. Kryzer and V. A. Lennon (2010). Eliminating false-positive results in serum tests for neuromuscular autoimmunity. Muscle Nerve 41(5): 702-4.

Barbara, G., V. Stanghellini, R. De Giorgio, C. Cremon, G. S. Cottrell, D. Santini, G. Pasquinelli, A. M. Morselli-Labate, E. F. Grady, N. W. Bunnett, S. M. Collins and R. Corinaldesi (2004). Activated mast cells in proximity to colonic nerves correlate with abdominal pain in irritable bowel syndrome. Gastroenterology 126(3): 693-702.

Barbara, G., B. A. Vallance and S. M. Collins (1997). Persistent intestinal neuromuscular dysfunction after acute nematode infection in mice. Gastroenterology 113(4): 1224-32.

Baroni, S. S., M. Santillo, F. Bevilacqua, M. Luchetti, T. Spadoni, M. Mancini, P. Fraticelli, P. Sambo, A. Funaro, A. Kazlauskas, E. V. Avvedimento and A. Gabrielli (2006). Stimulatory autoantibodies to the PDGF receptor in systemic sclerosis. N Engl J Med 354(25): 2667-76.

Battle, W. M., W. J. Snape, Jr., S. Wright, M. A. Sullivan, S. Cohen, A. Meyers and R. Tuthill (1981). Abnormal colonic motility in progressive systemic sclerosis. Ann Intern Med 94(6): 749-52.

Bean, B. P. (2007). The action potential in mammalian central neurons. Nat Rev Neurosci 8(6): 451-65. 
Beckh, S. and O. Pongs (1990). Members of the RCK potassium channel family are differentially expressed in the rat nervous system. EMBO J 9(3): 777-82.

Bell, C. E., Jr., S. Seetharam and R. C. McDaniel (1976). Endodermally-derived and neural crest-derived differentiation antigens expressed by a human lung tumor. $J$ Immunol 116(5): 1236-43.

Benatar, M. (2000). Neurological potassium channelopathies. QJM 93(12): 787-97.

Berry, M. S. (1972). A system of electrically coupled small cells in the buccal ganglia of the pond snail Planorbis corneus. J Exp Biol 56(3): 621-37.

Bischofberger, J., J. R. Geiger and P. Jonas (2002). Timing and efficacy of Ca2+ channel activation in hippocampal mossy fiber boutons. J Neurosci 22(24): 10593-602.

Blaes, F., D. Beeson, P. Plested, B. Lang and A. Vincent (2000). IgG from "seronegative" myasthenia gravis patients binds to a muscle cell line, TE671, but not to human acetylcholine receptor. Ann Neurol 47(4): 504-10.

Bodian, M., F. D. Stephens and B. C. Ward (1949). Hirschsprung's disease and idiopathic megacolon. Lancet 1(6540): 6-11.

Boeckxstaens, G. E. (2006). Novel mechanism for impaired nitrergic relaxation in achalasia. Gut 55(3): 304-5.

Bruley des Varannes, S., J. Chevalier, S. Pimont, J. C. Le Neel, M. Klotz, K. H. Schafer, J. P. Galmiche and M. Neunlist (2006). Serum from achalasia patients alters neurochemical coding in the myenteric plexus and nitric oxide mediated motor response in normal human fundus. Gut 55(3): 319-26.

Brundel, B. J., I. C. Van Gelder, R. H. Henning, A. E. Tuinenburg, M. Wietses, J. G. Grandjean, A. A. Wilde, W. H. Van Gilst and H. J. Crijns (2001). Alterations in potassium channel gene expression in atria of patients with persistent and paroxysmal atrial fibrillation: differential regulation of protein and mRNA levels for $\mathrm{K}+$ channels. $\mathrm{J}$ Am Coll Cardiol 37(3): 926-32.

Buckley, C., J. Oger, L. Clover, E. Tuzun, K. Carpenter, M. Jackson and A. Vincent (2001). Potassium channel antibodies in two patients with reversible limbic encephalitis. Ann Neurol 50(1): 73-8.

Budde-Steffen, C., N. E. Anderson, M. K. Rosenblum, F. Graus, D. Ford, B. J. Synek, S. H. Wray and J. B. Posner (1988). An antineuronal autoantibody in paraneoplastic opsoclonus. Ann Neurol 23(5): 528-31.

Bulbring, E. and T. Tomita (1967). Properties of the inhibitory potential of smooth muscle as observed in the response to field stimulation of the guinea-pig taenia coli. $J$ Physiol 189(2): 299-315.

Burry, R. W. (2000). Specificity controls for immunocytochemical methods. J Histochem Cytochem 48(2): 163-6.

Carew, T. J. and E. R. Kandel (1977). Inking in Aplysia californica. II. Central program for inking. J Neurophysiol 40(3): 708-20. 
Carl, A. (1995). Multiple components of delayed rectifier $\mathrm{K}+$ current in canine colonic smooth muscle. J Physiol 484 ( Pt 2): 339-53.

Castell, D. O. (1976). Achalasia and diffuse esophageal spasm. Arch Intern Med 136(5): 571-9.

Castle, N. A., D. G. Haylett and D. H. Jenkinson (1989). Toxins in the characterization of potassium channels. Trends Neurosci 12(2): 59-65.

Cervero, F. and J. M. Laird (2003). Role of ion channels in mechanisms controlling gastrointestinal pain pathways. Curr Opin Pharmacol 3(6): 608-12.

Chadwick, V. S., W. Chen, D. Shu, B. Paulus, P. Bethwaite, A. Tie and I. Wilson (2002). Activation of the mucosal immune system in irritable bowel syndrome. Gastroenterology 122(7): 1778-83.

Chagas, C. (1909). Nova tripanosomiaze humana. Mem Inst Oswaldo Cruz 1: 159-218.

Chan, K. H., D. H. Lachance, C. M. Harper and V. A. Lennon (2007). Frequency of seronegativity in adult-acquired generalized myasthenia gravis. Muscle Nerve 36(5): 6518 .

Chandrasekharan, B. and S. Srinivasan (2007). Diabetes and the enteric nervous system. Neurogastroenterol Motil 19(12): 951-60.

Chinn, J. S. and M. D. Schuffler (1988). Paraneoplastic visceral neuropathy as a cause of severe gastrointestinal motor dysfunction. Gastroenterology 95(5): 1279-86.

Chiu, S. Y. and J. M. Ritchie (1982). Evidence for the presence of potassium channels in the internode of frog myelinated nerve fibres. J Physiol 322: 485-501.

Christie, M. J., R. A. North, P. B. Osborne, J. Douglass and J. P. Adelman (1990). Heteropolymeric potassium channels expressed in Xenopus oocytes from cloned subunits. Neuron 4(3): 405-11.

Chung, Y. H., C. Shin, M. J. Kim, B. K. Lee and C. I. Cha (2001). Immunohistochemical study on the distribution of six members of the Kvl channel subunits in the rat cerebellum. Brain Res 895(1-2): 173-7.

Clark, S. B., T. W. Rice, R. R. Tubbs, J. E. Richter and J. R. Goldblum (2000). The nature of the myenteric infiltrate in achalasia: an immunohistochemical analysis. Am J Surg Pathol 24(8): 1153-8.

Cohen, S., R. Fisher, W. Lipshutz, R. Turner, A. Myers and R. Schumacher (1972). The pathogenesis of esophageal dysfunction in scleroderma and Raynaud's disease. J Clin Invest 51(10): 2663-8.

Coleman, S. K., J. Newcombe, J. Pryke and J. O. Dolly (1999). Subunit composition of Kv1 channels in human CNS. J Neurochem 73(2): 849-58.

Collins, S. M. (1996). The immunomodulation of enteric neuromuscular function: implications for motility and inflammatory disorders. Gastroenterology 111(6): 1683-99. 
Coons, A. H. and M. H. Kaplan (1950). Localization of antigen in tissue cells; improvements in a method for the detection of antigen by means of fluorescent antibody. J Exp Med 91(1): 1-13.

Cooper, E. C., A. Milroy, Y. N. Jan, L. Y. Jan and D. H. Lowenstein (1998). Presynaptic localization of Kv1.4-containing A-type potassium channels near excitatory synapses in the hippocampus. J Neurosci 18(3): 965-74.

Corbett, C. E., U. Ribeiro, Jr., M. G. Prianti, A. Habr-Gama, M. Okumura and J. GamaRodrigues (2001). Cell-mediated immune response in megacolon from patients with chronic Chagas' disease. Dis Colon Rectum 44(7): 993-8.

Costagliola, A., L. Van Nassauw, D. Snyders, D. Adriaensen and J. P. Timmermans (2009). Voltage-gated delayed rectifier K v 1-subunits may serve as distinctive markers for enteroglial cells with different phenotypes in the murine ileum. Neurosci Lett 461(2): $80-4$.

Csendes, A., G. Smok, I. Braghetto, P. Gonzalez, A. Henriquez, P. Csendes and D. Pizurno (1992). Histological studies of Auerbach's plexuses of the oesophagus, stomach, jejunum, and colon in patients with achalasia of the oesophagus: correlation with gastric acid secretion, presence of parietal cells and gastric emptying of solids. Gut 33(2): 150-4.

da Silveira, A. B., D. D'Avila Reis, E. C. de Oliveira, S. G. Neto, A. O. Luquetti, D. Poole, R. Correa-Oliveira and J. B. Furness (2007). Neurochemical coding of the enteric nervous system in chagasic patients with megacolon. Dig Dis Sci 52(10): 2877-83.

da Silveira, A. B., E. M. Lemos, S. J. Adad, R. Correa-Oliveira, J. B. Furness and D. D'Avila Reis (2007). Megacolon in Chagas disease: a study of inflammatory cells, enteric nerves, and glial cells. Hum Pathol 38(8): 1256-64.

Dang, K., K. Bielefeldt and G. F. Gebhart (2004). Gastric ulcers reduce A-type potassium currents in rat gastric sensory ganglion neurons. Am J Physiol Gastrointest Liver Physiol 286(4): G573-9.

Dau, P. C., M. B. Kahaleh and R. W. Sagebiel (1981). Plasmapheresis and immunosuppressive drug therapy in scleroderma. Arthritis Rheum 24(9): 1128-36.

Davison, P. F. and E. W. Taylor (1960). Physical-chemical studies of proteins of squid nerve axoplasm, with special reference to the axon fibrous protein. J Gen Physiol 43: 801-23.

De Giorgio, R., G. Barbara, V. Stanghellini, F. De Ponti, B. Salvioli, M. Tonini, P. Velio, G. Bassotti and R. Corinaldesi (2002). Clinical and morphofunctional features of idiopathic myenteric ganglionitis underlying severe intestinal motor dysfunction: a study of three cases. Am J Gastroenterol 97(9): 2454-9.

De Giorgio, R., M. Bovara, G. Barbara, M. Canossa, G. Sarnelli, F. De Ponti, V. Stanghellini, M. Tonini, S. Cappello, E. Pagnotta, E. Nobile-Orazio and R. Corinaldesi (2003). Anti-HuD-induced neuronal apoptosis underlying paraneoplastic gut dysmotility. Gastroenterology 125(1): 70-9. 
De Giorgio, R., M. P. Di Simone, V. Stanghellini, G. Barbara, M. Tonini, B. Salvioli, S. Mattioli and R. Corinaldesi (1999). Esophageal and gastric nitric oxide synthesizing innervation in primary achalasia. Am J Gastroenterol 94(9): 2357-62.

de Giorgio, R., U. Volta, V. Stanghellini, R. F. Cogliandro, G. Barbara, R. Corinaldesi, R. Towns, C. Guo, S. Hong and J. W. Wiley (2008). Neurogenic chronic intestinal pseudo-obstruction: antineuronal antibody-mediated activation of autophagy via Fas. Gastroenterology 135(2): 601-9.

de Scheerder, I. K., M. L. de Buyzere, J. R. Delanghe, D. L. Clement and R. J. Wieme (1989). Anti-myosin humoral immune response following cardiac injury. Autoimmunity 4(1-2): 51-8.

Dewit, S., G. de Hertogh, K. Geboes and J. Tack (2008). Chronic intestinal pseudoobstruction caused by an intestinal inflammatory myopathy: case report and review of the literature. Neurogastroenterol Motil 20(4): 343-8.

Dhamija, R., K. M. Tan, S. J. Pittock, A. Foxx-Orenstein, E. Benarroch and V. A. Lennon (2008). Serologic profiles aiding the diagnosis of autoimmune gastrointestinal dysmotility. Clin Gastroenterol Hepatol 6(9): 988-92.

Di Lorenzo, C. (1999). Pseudo-obstruction: current approaches. Gastroenterology 116(4): 980-7.

Diepholder, H. M., K. Schwechheimer, M. Mohadjer, R. Knoth and B. Volk (1991). A clinicopathologic and immunomorphologic study of 13 cases of ganglioglioma. Cancer 68(10): 2192-201.

Drachman, D. B., C. W. Angus, R. N. Adams, J. D. Michelson and G. J. Hoffman (1978). Myasthenic antibodies cross-link acetylcholine receptors to accelerate degradation. N Engl J Med 298(20): 1116-22.

Dudley, H. A., I. S. Sinclair, L. I. Mc, N. T. Mc and J. E. Newsam (1958). Intestinal pseudo-obstruction. J R Coll Surg Edinb 3(3): 206-17.

Eaker, E. Y., J. G. Kuldau, G. N. Verne, S. O. Ross and J. E. Sallustio (1999). Myenteric neuronal antibodies in scleroderma: passive transfer evokes alterations in intestinal myoelectric activity in a rat model. J Lab Clin Med 133(6): 551-6.

Elizari, M. V. (2002). Arrhythmias associated with Chagas' heart disease. Card Electrophysiol Rev 6(1-2): 115-9.

Elmqvist, D. and E. H. Lambert (1968). Detailed analysis of neuromuscular transmission in a patient with the myasthenic syndrome sometimes associated with bronchogenic carcinoma. Mayo Clin Proc 43(10): 689-713.

Engel, A. G. (1979). The immunopathological basis of acetylcholine receptor deficiency in myasthenia gravis. Prog Brain Res 49: 423-34.

Engel, A. G. and K. Arahata (1987). The membrane attack complex of complement at the endplate in myasthenia gravis. Ann N Y Acad Sci 505: 326-32. 
Engel, A. G., E. H. Lambert and F. M. Howard (1977). Immune complexes (IgG and C3) at the motor end-plate in myasthenia gravis: ultrastructural and light microscopic localization and electrophysiologic correlations. Mayo Clin Proc 52(5): 267-80.

Engel, A. G., K. Sahashi and G. Fumagalli (1981). The immunopathology of acquired myasthenia gravis. Ann N Y Acad Sci 377: 158-74.

Epperson, A., H. P. Bonner, S. M. Ward, W. J. Hatton, K. K. Bradley, M. E. Bradley, J. S. Trimmer and B. Horowitz (1999). Molecular diversity of K(V) alpha- and beta-subunit expression in canine gastrointestinal smooth muscles. Am J Physiol 277(1 Pt 1): G12736.

Farrugia, G. (2008). Interstitial cells of Cajal in health and disease. Neurogastroenterol Motil 20 Suppl 1: 54-63.

Farrugia, G., A. Rich, J. L. Rae, M. G. Sarr and J. H. Szurszewski (1995). Calcium currents in human and canine jejunal circular smooth muscle cells. Gastroenterology 109(3): 707-17.

Feng, J., B. Wible, G. R. Li, Z. Wang and S. Nattel (1997). Antisense oligodeoxynucleotides directed against Kv1.5 mRNA specifically inhibit ultrarapid delayed rectifier $\mathrm{K}+$ current in cultured adult human atrial myocytes. Circ Res 80(4): 572-9.

Ferreira-Santos, R. (1961). Aperistalsis of the esophagus and colon (megaesophagus and megacolon) etiologically related to Chagas' disease. Am J Dig Dis 6: 700-26.

Franklin, J. L. and A. L. Willard (1993). Voltage-dependent sodium and calcium currents of rat myenteric neurons in cell culture. J Neurophysiol 69(4): 1264-75.

Fu, L. X., Y. Magnusson, C. H. Bergh, J. A. Liljeqvist, F. Waagstein, A. Hjalmarson and J. Hoebeke (1993). Localization of a functional autoimmune epitope on the muscarinic acetylcholine receptor-2 in patients with idiopathic dilated cardiomyopathy. J Clin Invest 91(5): 1964-8.

Fukuoka, T., A. G. Engel, B. Lang, J. Newsom-Davis, C. Prior and D. W. Wray (1987). Lambert-Eaton myasthenic syndrome: I. Early morphological effects of $\operatorname{IgG}$ on the presynaptic membrane active zones. Ann Neurol 22(2): 193-9.

Furness, J. B. (2000). Types of neurons in the enteric nervous system. J Auton Nerv Syst 81(1-3): 87-96.

Galligan, J. J. (2002). Ligand-gated ion channels in the enteric nervous system. Neurogastroenterol Motil 14(6): 611-23.

Ganzinelli, S., E. Borda, L. Joensen and L. Sterin-Borda (2009). Chagasic antibodies induce cardiac COX-2/iNOS mRNA expression with PGE2/NO production. Int J Cardiol 134(2): 212-23.

Gasparini, S., J. M. Danse, A. Lecoq, S. Pinkasfeld, S. Zinn-Justin, L. C. Young, C. C. de Medeiros, E. G. Rowan, A. L. Harvey and A. Menez (1998). Delineation of the functional site of alpha-dendrotoxin. The functional topographies of dendrotoxins are 
different but share a conserved core with those of other Kv1 potassium channel-blocking toxins. J Biol Chem 273(39): 25393-403.

Geiger, J. R. and P. Jonas (2000). Dynamic control of presynaptic $\mathrm{Ca}(2+)$ inflow by fastinactivating $\mathrm{K}(+)$ channels in hippocampal mossy fiber boutons. Neuron 28(3): 927-39.

Gerl, A., M. Storck, A. Schalhorn, J. Muller-Hocker, K. W. Jauch, F. W. Schildberg and W. Wilmanns (1992). Paraneoplastic chronic intestinal pseudoobstruction as a rare complication of bronchial carcinoid. Gut 33(7): 1000-3.

Gershon, M. D. and S. M. Erde (1981). The nervous system of the gut. Gastroenterology 80(6): 1571-94.

Ghia, J. E., F. Crenner, S. Rohr, C. Meyer, M. H. Metz-Boutigue, D. Aunis and F. Angel (2004). A role for chromogranin A (4-16), a vasostatin-derived peptide, on human colonic motility. An in vitro study. Regul Pept 121(1-3): 31-9.

Ghirardo, S., B. Sauter, G. Levy, I. M. Fiel, T. Schiano and G. Gondolesi (2005). Primary intestinal autoimmune disease as a cause of chronic intestinal pseudo-obstruction. Gut 54(8): 1206-7.

Gibbons, J. C. and J. F. Sullivan (1978). Chronic idiopathic pseudo-obstructive bowel disease. Am J Gastroenterol 70(3): 306-13.

Gilchrist, A. M., J. O. Mills and C. G. Russell (1985). Acute large-bowel pseudoobstruction. Clin Radiol 36(4): 401-4.

Glazebrook, P. A., A. N. Ramirez, J. H. Schild, C. C. Shieh, T. Doan, B. A. Wible and D. L. Kunze (2002). Potassium channels Kv1.1, Kv1.2 and Kv1.6 influence excitability of rat visceral sensory neurons. J Physiol 541(Pt 2): 467-82.

Goin, J. C., E. Borda, C. P. Leiros, R. Storino and L. Sterin-Borda (1994). Identification of antibodies with muscarinic cholinergic activity in human Chagas' disease: pathological implications. J Auton Nerv Syst 47(1-2): 45-52.

Goin, J. C., C. P. Leiros, E. Borda and L. Sterin-Borda (1997). Interaction of human chagasic IgG with the second extracellular loop of the human heart muscarinic acetylcholine receptor: functional and pathological implications. FASEB J 11(1): 77-83.

Goin, J. C., C. Perez Leiros, E. Borda and L. Sterin-Borda (1994). Modification of cholinergic-mediated cellular transmembrane signals by the interaction of human chagasic IgG with cardiac muscarinic receptors. Neuroimmunomodulation 1(5): 284-91.

Goin, J. C., L. Sterin-Borda, C. R. Bilder, L. M. Varrica, G. Iantorno, M. C. Rios and E. Borda (1999). Functional implications of circulating muscarinic cholinergic receptor autoantibodies in chagasic patients with achalasia. Gastroenterology 117(4): 798-805.

Goldblatt, F., T. P. Gordon and S. A. Waterman (2002). Antibody-mediated gastrointestinal dysmotility in scleroderma. Gastroenterology 123(4): 1144-50.

Goldblum, J. R., R. I. Whyte, M. B. Orringer and H. D. Appelman (1994). Achalasia. A morphologic study of 42 resected specimens. Am J Surg Pathol 18(4): 327-37. 
Gomez, A., F. Martos, I. Bellido, E. Marquez, A. J. Garcia, J. Pavia and F. Sanchez de la Cuesta (1992). Muscarinic receptor subtypes in human and rat colon smooth muscle. Biochem Pharmacol 43(11): 2413-9.

Gonsalkorale, W. M., C. Perrey, V. Pravica, P. J. Whorwell and I. V. Hutchinson (2003). Interleukin 10 genotypes in irritable bowel syndrome: evidence for an inflammatory component? Gut 52(1): 91-3.

Graus, F., K. B. Elkon, C. Cordon-Cardo and J. B. Posner (1986). Sensory neuronopathy and small cell lung cancer. Antineuronal antibody that also reacts with the tumor. Am J Med 80(1): 45-52.

Graus, F., O. G. Segurado and E. Tolosa (1988). Selective concentration of anti-Purkinje cell antibody in the CSF of two patients with paraneoplastic cerebellar degeneration. Acta Neurol Scand 78(3): 210-3.

Greenlee, J. E. and H. R. Brashear (1983). Antibodies to cerebellar Purkinje cells in patients with paraneoplastic cerebellar degeneration and ovarian carcinoma. Ann Neurol 14(6): 609-13.

Grissmer, S., A. N. Nguyen, J. Aiyar, D. C. Hanson, R. J. Mather, G. A. Gutman, M. J. Karmilowicz, D. D. Auperin and K. G. Chandy (1994). Pharmacological characterization of five cloned voltage-gated $\mathrm{K}+$ channels, types $\mathrm{Kv1} 1.1,1.2,1.3,1.5$, and 3.1 , stably expressed in mammalian cell lines. Mol Pharmacol 45(6): 1227-34.

Gutman, G. A., K. G. Chandy, S. Grissmer, M. Lazdunski, D. McKinnon, L. A. Pardo, G. A. Robertson, B. Rudy, M. C. Sanguinetti, W. Stuhmer and X. Wang (2005). International Union of Pharmacology. LIII. Nomenclature and molecular relationships of voltage-gated potassium channels. Pharmacol Rev 57(4): 473-508.

Gwee, K. A. (2010). Post-Infectious Irritable Bowel Syndrome, an InflammationImmunological Model with Relevance for Other IBS and Functional Dyspepsia. J Neurogastroenterol Motil 16(1): 30-4.

Haas, S., L. Bindl and H. P. Fischer (2005). Autoimmune enteric leiomyositis: a rare cause of chronic intestinal pseudo-obstruction with specific morphological features. Hum Pathol 36(5): 576-80.

Hagger, R., C. Finlayson, F. Kahn, R. De Oliveira, L. Chimelli and D. Kumar (2000). A deficiency of interstitial cells of Cajal in Chagasic megacolon. J Auton Nerv Syst 80(12): 108-11.

Hart, I. K., P. Maddison, J. Newsom-Davis, A. Vincent and K. R. Mills (2002). Phenotypic variants of autoimmune peripheral nerve hyperexcitability. Brain 125(Pt 8): 1887-95.

Hart, I. K., C. Waters, A. Vincent, C. Newland, D. Beeson, O. Pongs, C. Morris and J. Newsom-Davis (1997). Autoantibodies detected to expressed $\mathrm{K}+$ channels are implicated in neuromyotonia. Ann Neurol 41(2): 238-46.

Hart, P. J., K. E. Overturf, S. N. Russell, A. Carl, J. R. Hume, K. M. Sanders and B. Horowitz (1993). Cloning and expression of a Kv1.2 class delayed rectifier K+ channel from canine colonic smooth muscle. Proc Natl Acad Sci U S A 90(20): 9659-63. 
Hatton, W. J., H. S. Mason, A. Carl, P. Doherty, M. J. Latten, J. L. Kenyon, K. M. Sanders and B. Horowitz (2001). Functional and molecular expression of a voltagedependent $\mathrm{K}(+)$ channel (Kv1.1) in interstitial cells of Cajal. J Physiol 533(Pt 2): 315-27.

He, C. L., L. Burgart, L. Wang, J. Pemberton, T. Young-Fadok, J. Szurszewski and G. Farrugia (2000). Decreased interstitial cell of cajal volume in patients with slow-transit constipation. Gastroenterology 118(1): 14-21.

Heanue, T. A. and V. Pachnis (2007). Enteric nervous system development and Hirschsprung's disease: advances in genetic and stem cell studies. Nat Rev Neurosci 8(6): 466-79.

Hernandez, C. C., L. C. Barcellos, L. E. Gimenez, R. A. Cabarcas, S. Garcia, R. C. Pedrosa, J. H. Nascimento, E. Kurtenbach, M. O. Masuda and A. C. Campos de Carvalho (2003). Human chagasic IgGs bind to cardiac muscarinic receptors and impair L-type $\mathrm{Ca} 2+$ currents. Cardiovasc Res 58(1): 55-65.

Hernandez, C. C., J. H. Nascimento, E. A. Chaves, P. C. Costa, M. O. Masuda, E. Kurtenbach, D. E. C. A. C. Campos and L. E. Gimenez (2008). Autoantibodies enhance agonist action and binding to cardiac muscarinic receptors in chronic Chagas' disease. $\mathrm{J}$ Recept Signal Transduct Res 28(4): 375-401.

Hertz, A. F. (1915). Achalasia of the Cardia (so-called Cardio-spasm). Proc R Soc Med 8(Clin Sect): 22-25.

Hille, B. (2001). Ion Channels of Excitable Membranes. Sunderland, Sinauer Associates.

Hirsh, E. H., D. Brandenburg, T. Hersh and W. S. Brooks, Jr. (1981). Chronic intestinal pseudo-obstruction. J Clin Gastroenterol 3(3): 247-54.

Hoch, W., J. McConville, S. Helms, J. Newsom-Davis, A. Melms and A. Vincent (2001). Auto-antibodies to the receptor tyrosine kinase MuSK in patients with myasthenia gravis without acetylcholine receptor antibodies. Nat Med 7(3): 365-8.

Hodgkin, A. L. and A. F. Huxley (1952). A quantitative description of membrane current and its application to conduction and excitation in nerve. J Physiol 117(4): 500-44.

Howe, S., E. Y. Eaker, J. E. Sallustio, C. Peebles, E. M. Tan and R. C. Williams, Jr. (1994). Antimyenteric neuronal antibodies in scleroderma. J Clin Invest 94(2): 761-70.

Huizinga, J. D. (1998). Neural injury, repair, and adaptation in the GI tract. IV. Pathophysiology of GI motility related to interstitial cells of Cajal. Am J Physiol 275(3 Pt 1): G381-6.

Huizinga, J. D., C. M. Golden, Y. Zhu and E. J. White (2004). Ion channels in interstitial cells of Cajal as targets for neurotransmitter action. Neurogastroenterol Motil 16 Suppl 1: 106-11.

Iantorno, G., G. Bassotti, Z. Kogan, C. M. Lumi, A. M. Cabanne, S. Fisogni, L. M. Varrica, C. R. Bilder, J. P. Munoz, B. Liserre, A. Morelli and V. Villanacci (2007). The enteric nervous system in chagasic and idiopathic megacolon. Am J Surg Pathol 31(3): 460-8. 
Iizuka, T., F. Sakai, T. Ide, T. Monzen, S. Yoshii, M. Iigaya, K. Suzuki, D. R. Lynch, N. Suzuki, T. Hata and J. Dalmau (2008). Anti-NMDA receptor encephalitis in Japan: longterm outcome without tumor removal. Neurology 70(7): 504-11.

Irani, S. R., K. Bera, P. Waters, L. Zuliani, S. Maxwell, M. S. Zandi, M. A. Friese, I. Galea, D. M. Kullmann, D. Beeson, B. Lang, C. G. Bien and A. Vincent (2010). Nmethyl-D-aspartate antibody encephalitis: temporal progression of clinical and paraclinical observations in a predominantly non-paraneoplastic disorder of both sexes. Brain 133(Pt 6): 1655-67.

Isaacs, H. (1961). A syndrome of continuous muscle fibre activity. J Neurol Neurosurg Psychiatry 24: 319-325.

Jackson, M. W., T. P. Gordon and S. A. Waterman (2004). Disruption of intestinal motility by a calcium channel-stimulating autoantibody in type 1 diabetes. Gastroenterology 126(3): 819-28.

Kao, C. Y. (1964). Tetrodotoxin: Mechanism of Action. Science 144: 319.

Kawaguchi, Y., Y. Nakamura, I. Matsumoto, E. Nishimagi, T. Satoh, M. Kuwana, T. Sumida and M. Hara (2009). Muscarinic-3 acetylcholine receptor autoantibody in patients with systemic sclerosis: contribution to severe gastrointestinal tract dysmotility. Ann Rheum Dis 68(5): 710-4.

Keshavarzian, A., F. L. Iber and J. Vaeth (1987). Gastric emptying in patients with insulin-requiring diabetes mellitus. Am J Gastroenterol 82(1): 29-35.

Kierszenbaum, F. (1999). Chagas' disease and the autoimmunity hypothesis. Clin Microbiol Rev 12(2): 210-23.

Kierszenbaum, F. (2005). Where do we stand on the autoimmunity hypothesis of Chagas disease? Trends Parasitol 21(11): 513-6.

Kim, Y. I., E. H. Middlekauff, M. P. Viglione, J. Okutsu, Y. Satoh, N. Hirashima and Y. Kirino (1998). An autoimmune animal model of the Lambert-Eaton syndrome. Ann N Y Acad Sci 841: 670-6.

Kirchhoff, L. V. (2003). Changing Epidemiology and Approaches to Therapy for Chagas Disease. Curr Infect Dis Rep 5(1): 59-65.

Kleopa, K. A., L. B. Elman, B. Lang, A. Vincent and S. S. Scherer (2006). Neuromyotonia and limbic encephalitis sera target mature Shaker-type K+ channels: subunit specificity correlates with clinical manifestations. Brain 129(Pt 6): 1570-84.

Knowles, C. H., R. De Giorgio, R. P. Kapur, E. Bruder, G. Farrugia, K. Geboes, M. D. Gershon, J. Hutson, G. Lindberg, J. E. Martin, W. A. Meier-Ruge, P. J. Milla, V. V. Smith, J. M. Vandervinden, B. Veress and T. Wedel (2009). Gastrointestinal neuromuscular pathology: guidelines for histological techniques and reporting on behalf of the Gastro 2009 International Working Group. Acta Neuropathol 118(2): 271-301.

Knowles, C. H., R. De Giorgio, R. P. Kapur, E. Bruder, G. Farrugia, K. Geboes, G. Lindberg, J. E. Martin, W. A. Meier-Ruge, P. J. Milla, V. V. Smith, J. M. Vandervinden, B. Veress and T. Wedel (2010). The London Classification of gastrointestinal 
neuromuscular pathology: report on behalf of the Gastro 2009 International Working Group. Gut 59(7): 882-7.

Knowles, C. H., B. Lang, L. Clover, S. M. Scott, C. Gotti, A. Vincent and J. E. Martin (2002). A role for autoantibodies in some cases of acquired non-paraneoplastic gut dysmotility. Scand J Gastroenterol 37(2): 166-70.

Knowles, C. H. and J. E. Martin (2000). Slow transit constipation: a model of human gut dysmotility. Review of possible aetiologies. Neurogastroenterol Motil 12(2): 181-96.

Knowles, C. H., S. M. Scott, A. Wellmer, V. P. Misra, M. A. Pilot, N. S. Williams and P. Anand (1999). Sensory and autonomic neuropathy in patients with idiopathic slow-transit constipation. Br J Surg 86(1): 54-60.

Knowles, C. H., D. B. Silk, A. Darzi, B. Veress, R. Feakins, A. H. Raimundo, T. Crompton, E. C. Browning, G. Lindberg and J. E. Martin (2004). Deranged smooth muscle alpha-actin as a biomarker of intestinal pseudo-obstruction: a controlled multinational case series. Gut 53(11): 1583-9.

Knowles, C. H., B. Veress, H. Tornblom, S. Wallace, P. Paraskeva, A. Darzi, J. E. Martin, B. Nyberg and G. Lindberg (2008). Safety and diagnostic yield of laparoscopically assisted full-thickness bowel biospy. Neurogastroenterol Motil.

Koberle, F. (1968). Chagas' disease and Chagas' syndromes: the pathology of American trypanosomiasis. Adv Parasitol 6: 63-116.

Koh, S. D., S. M. Ward, G. M. Dick, A. Epperson, H. P. Bonner, K. M. Sanders, B. Horowitz and J. L. Kenyon (1999). Contribution of delayed rectifier potassium currents to the electrical activity of murine colonic smooth muscle. J Physiol 515 ( Pt 2): 475-87.

Kong, X. C., P. Barzaghi and M. A. Ruegg (2004). Inhibition of synapse assembly in mammalian muscle in vivo by RNA interference. EMBO Rep 5(2): 183-8.

Kosterlitz, H. W., R. J. Lydon and A. J. Watt (1970). The effects of adrenaline, noradrenaline and isoprenaline on inhibitory alpha- and beta-adrenoceptors in the longitudinal muscle of the guinea-pig ileum. Br J Pharmacol 39(2): 398-413.

Kraichely, R. E., G. Farrugia, S. J. Pittock, D. O. Castell and V. A. Lennon (2009). Neural Autoantibody Profile of Primary Achalasia. Dig Dis Sci.

Lai, L. P., M. J. Su, J. L. Lin, F. Y. Lin, C. H. Tsai, Y. S. Chen, Y. Z. Tseng, W. P. Lien and S. K. Huang (1999). Changes in the mRNA levels of delayed rectifier potassium channels in human atrial fibrillation. Cardiology 92(4): 248-55.

Lang, B., R. C. Dale and A. Vincent (2003). New autoantibody mediated disorders of the central nervous system. Curr Opin Neurol 16(3): 351-7.

Lang, B., J. Newsom-Davis, C. Peers, C. Prior and D. W. Wray (1987). The effect of myasthenic syndrome antibody on presynaptic calcium channels in the mouse. J Physiol 390: $257-70$.

Latiano, A., R. De Giorgio, U. Volta, O. Palmieri, C. Zagaria, V. Stanghellini, G. Barbara, A. Mangia, A. Andriulli, R. Corinaldesi and V. Annese (2006). HLA and enteric antineuronal antibodies in patients with achalasia. Neurogastroenterol Motil 18(7): 520-5. 
Lee, H. R., V. A. Lennon, M. Camilleri and C. M. Prather (2001). Paraneoplastic gastrointestinal motor dysfunction: clinical and laboratory characteristics. Am J Gastroenterol 96(2): 373-9.

Legge, D. A., E. E. Wollaeger and H. C. Carlson (1970). Intestinal pseudo-obstruction in systemic amyloidosis. Gut 11(9): 764-7.

Leite, M. I., S. Jacob, S. Viegas, J. Cossins, L. Clover, B. P. Morgan, D. Beeson, N. Willcox and A. Vincent (2008). IgG1 antibodies to acetylcholine receptors in 'seronegative' myasthenia gravis. Brain 131(Pt 7): 1940-52.

Lennon, V. A., L. G. Ermilov, J. H. Szurszewski and S. Vernino (2003). Immunization with neuronal nicotinic acetylcholine receptor induces neurological autoimmune disease. J Clin Invest 111(6): 907-13.

Lennon, V. A., T. J. Kryzer, G. E. Griesmann, P. E. O'Suilleabhain, A. J. Windebank, A. Woppmann, G. P. Miljanich and E. H. Lambert (1995). Calcium-channel antibodies in the Lambert-Eaton syndrome and other paraneoplastic syndromes. N Engl J Med 332(22): 1467-74.

Lennon, V. A., T. J. Kryzer, S. J. Pittock, A. S. Verkman and S. R. Hinson (2005). IgG marker of optic-spinal multiple sclerosis binds to the aquaporin-4 water channel. J Exp Med 202(4): 473-7.

Lennon, V. A., D. F. Sas, M. F. Busk, B. Scheithauer, J. R. Malagelada, M. Camilleri and L. J. Miller (1991). Enteric neuronal autoantibodies in pseudoobstruction with small-cell lung carcinoma. Gastroenterology 100(1): 137-42.

Lescure, F. X., G. Le Loup, H. Freilij, M. Develoux, L. Paris, L. Brutus and G. Pialoux (2010). Chagas disease: changes in knowledge and management. Lancet Infect Dis 10(8): $556-570$.

Liimatainen, S., M. Peltola, L. Sabater, M. Fallah, E. Kharazmi, A. M. Haapala, P. Dastidar, M. Knip, A. Saiz and J. Peltola (2010). Clinical significance of glutamic acid decarboxylase antibodies in patients with epilepsy. Epilepsia 51(5): 760-7.

Lindberg, G., M. Iwarzon and H. Tornblom (2009). Clinical features and long-term survival in chronic intestinal pseudo-obstruction and enteric dysmotility. Scand $\mathbf{J}$ Gastroenterol 44(6): 692-9.

Lindberg, G., H. Tornblom, M. Iwarzon, B. Nyberg, J. E. Martin and B. Veress (2009). Full-thickness biopsy findings in chronic intestinal pseudo-obstruction and enteric dysmotility. Gut 58(8): 1084-90.

Lindstrom, J. M., M. E. Seybold, V. A. Lennon, S. Whittingham and D. D. Duane (1976). Antibody to acetylcholine receptor in myasthenia gravis. Prevalence, clinical correlates, and diagnostic value. Neurology 26(11): 1054-9.

Liu, X., N. J. Rusch, J. Striessnig and S. K. Sarna (2001). Down-regulation of L-type calcium channels in inflamed circular smooth muscle cells of the canine colon. Gastroenterology 120(2): 480-9. 
Llinas, R., M. Sugimori, J. W. Lin and B. Cherksey (1989). Blocking and isolation of a calcium channel from neurons in mammals and cephalopods utilizing a toxin fraction (FTX) from funnel-web spider poison. Proc Natl Acad Sci U S A 86(5): 1689-93.

Lucchinetti, C. F., D. W. Kimmel and V. A. Lennon (1998). Paraneoplastic and oncologic profiles of patients seropositive for type 1 antineuronal nuclear autoantibodies. Neurology 50(3): 652-7.

Luider, T. M., M. W. van Dommelen, D. Tibboel, J. H. Meijers, F. J. Ten Kate, J. Q. Trojanowski and J. C. Molenaar (1992). Differences in phosphorylation state of neurofilament proteins in ganglionic and aganglionic bowel segments of children with Hirschsprung's disease. J Pediatr Surg 27(7): 815-9.

Luque, F. A., H. M. Furneaux, R. Ferziger, M. K. Rosenblum, S. H. Wray, S. C. Schold, Jr., M. J. Glantz, K. A. Jaeckle, H. Biran, M. Lesser and et al. (1991). Anti-Ri: an antibody associated with paraneoplastic opsoclonus and breast cancer. Ann Neurol 29(3): 241-51.

Ma, M. and J. Koester (1995). Consequences and mechanisms of spike broadening of R20 cells in Aplysia californica. J Neurosci 15(10): 6720-34.

MacKinnon, R. (1991). Determination of the subunit stoichiometry of a voltage-activated potassium channel. Nature 350(6315): 232-5.

Martin, A., A. Messineo, P. Lionetti, U. Volta, V. Stanghellini, G. Barbara, R. Cogliandro, R. Corinaldesi and R. De Giorgio (2008). A case of paraneoplastic inflammatory neuropathy of the gastrointestinal tract related to an underlying neuroblastoma: successful management with immunosuppressive therapy. J Pediatr Gastroenterol Nutr 46(4): 457-60.

Maselli, R. A., D. P. Richman and R. L. Wollmann (1991). Inflammation at the neuromuscular junction in myasthenia gravis. Neurology 41(9): 1497-504.

McDaniel, S. S., O. Platoshyn, Y. Yu, M. Sweeney, V. A. Miriel, V. A. Golovina, S. Krick, B. R. Lapp, J. Y. Wang and J. X. Yuan (2001). Anorexic effect of K+ channel blockade in mesenteric arterial smooth muscle and intestinal epithelial cells. J Appl Physiol 91(5): 2322-33.

Mearin, F., M. Mourelle, F. Guarner, A. Salas, V. Riveros-Moreno, S. Moncada and J. R. Malagelada (1993). Patients with achalasia lack nitric oxide synthase in the gastrooesophageal junction. Eur J Clin Invest 23(11): 724-8.

Meriney, S. D., S. C. Hulsizer, V. A. Lennon and A. D. Grinnell (1996). Lambert-Eaton myasthenic syndrome immunoglobulins react with multiple types of calcium channels in small-cell lung carcinoma. Ann Neurol 40(5): 739-49.

Miles, M. A., M. S. Llewellyn, M. D. Lewis, M. Yeo, R. Baleela, S. Fitzpatrick, M. W. Gaunt and I. L. Mauricio (2009). The molecular epidemiology and phylogeography of Trypanosoma cruzi and parallel research on Leishmania: looking back and to the future. Parasitology 136(12): 1509-28.

Moczydlowski, E., K. Lucchesi and A. Ravindran (1988). An emerging pharmacology of peptide toxins targeted against potassium channels. J Membr Biol 105(2): 95-111. 
Modarressi, M. H., B. Behnam, M. Cheng, K. E. Taylor, J. Wolfe and F. A. van der Hoorn (2004). Tsga10 encodes a 65-kilodalton protein that is processed to the 27kilodalton fibrous sheath protein. Biol Reprod 70(3): 608-15.

Moses, P. L., L. M. Ellis, M. R. Anees, W. Ho, R. I. Rothstein, J. B. Meddings, K. A. Sharkey and G. M. Mawe (2003). Antineuronal antibodies in idiopathic achalasia and gastro-oesophageal reflux disease. Gut 52(5): 629-36.

Motomura, M., B. Lang, I. Johnston, J. Palace, A. Vincent and J. Newsom-Davis (1997). Incidence of serum anti-P/O-type and anti-N-type calcium channel autoantibodies in the Lambert-Eaton myasthenic syndrome. J Neurol Sci 147(1): 35-42.

Nagado, T., K. Arimura, Y. Sonoda, A. Kurono, Y. Horikiri, A. Kameyama, M. Kameyama, O. Pongs and M. Osame (1999). Potassium current suppression in patients with peripheral nerve hyperexcitability. Brain 122 ( Pt 11): 2057-66.

Narahashi, T., T. Deguchi, N. Urakawa and Y. Ohkubo (1960). Stabilization and rectification of muscle fiber membrane by tetrodotoxin. Am J Physiol 198: 934-8.

Newsom-Davis, J. and K. R. Mills (1993). Immunological associations of acquired neuromyotonia (Isaacs' syndrome). Report of five cases and literature review. Brain 116 ( Pt 2): 453-69.

Newsom-Davis, J. and N. M. Murray (1984). Plasma exchange and immunosuppressive drug treatment in the Lambert-Eaton myasthenic syndrome. Neurology 34(4): 480-5.

Newsom-Davis, J., A. J. Pinching, A. Vincent and S. G. Wilson (1978). Function of circulating antibody to acetylcholine receptor in myasthenia gravis: investigation by plasma exchange. Neurology 28(3): 266-72.

Newsom-Davis, J. and A. Vincent (1991). Antibody-mediated neurological disease. Curr Opin Neurobiol 1(3): 430-5.

North, R. A., B. E. Slack and A. Surprenant (1985). Muscarinic M1 and M2 receptors mediate depolarization and presynaptic inhibition in guinea-pig enteric nervous system. J Physiol 368: 435-52.

O'Mahony, L., J. McCarthy, P. Kelly, G. Hurley, F. Luo, K. Chen, G. C. O'Sullivan, B. Kiely, J. K. Collins, F. Shanahan and E. M. Quigley (2005). Lactobacillus and bifidobacterium in irritable bowel syndrome: symptom responses and relationship to cytokine profiles. Gastroenterology 128(3): 541-51.

Pan, H., H. Y. Wang, E. Friedman and M. D. Gershon (1997). Mediation by protein kinases $\mathrm{C}$ and $\mathrm{A}$ of Go-linked slow responses of enteric neurons to 5-HT. J Neurosci 17(3): 1011-24.

Pande, R. and A. A. Leis (1999). Myasthenia gravis, thymoma, intestinal pseudoobstruction, and neuronal nicotinic acetylcholine receptor antibody. Muscle Nerve 22(11): 1600-2.

Pandolfino, J. E. and P. J. Kahrilas (2005). AGA technical review on the clinical use of esophageal manometry. Gastroenterology 128(1): 209-24. 
Parcej, D. N., V. E. Scott and J. O. Dolly (1992). Oligomeric properties of alphadendrotoxin-sensitive potassium ion channels purified from bovine brain. Biochemistry 31(45): 11084-8.

Pardi, D. S., S. M. Miller, D. L. Miller, L. J. Burgart, J. H. Szurszewski, V. A. Lennon and G. Farrugia (2002). Paraneoplastic dysmotility: loss of interstitial cells of Cajal. Am J Gastroenterol 97(7): 1828-33.

Pardo, L. A., S. H. Heinemann, H. Terlau, U. Ludewig, C. Lorra, O. Pongs and W. Stuhmer (1992). Extracellular K+ specifically modulates a rat brain $\mathrm{K}+$ channel. Proc Natl Acad Sci U S A 89(6): 2466-70.

Park, W. and M. F. Vaezi (2005). Etiology and pathogenesis of achalasia: the current understanding. Am J Gastroenterol 100(6): 1404-14.

Pasha, S. F., T. N. Lunsford and V. A. Lennon (2006). Autoimmune gastrointestinal dysmotility treated successfully with pyridostigmine. Gastroenterology 131(5): 1592-6.

Patrick, J. and J. Lindstrom (1973). Autoimmune response to acetylcholine receptor. Science 180(88): 871-2.

Perez, A. R., G. H. Fontanella, A. L. Nocito, S. Revelli and O. A. Bottasso (2009). Short treatment with the tumour necrosis factor-alpha blocker infliximab diminishes chronic chagasic myocarditis in rats without evidence of Trypanosoma cruzi reactivation. Clin Exp Immunol 157(2): 291-9.

Peter, J. C., J. Tugler, P. Eftekhari, D. Maurice, J. Hoebeke and J. C. Roegel (2005). Effects on heart rate of an anti-M2 acetylcholine receptor immune response in mice. FASEB J 19(8): 943-9.

Pinto, A., S. Gillard, F. Moss, K. Whyte, P. Brust, M. Williams, K. Stauderman, M. Harpold, B. Lang, J. Newsom-Davis, D. Bleakman, D. Lodge and J. Boot (1998). Human autoantibodies specific for the alpha1A calcium channel subunit reduce both P-type and Q-type calcium currents in cerebellar neurons. Proc Natl Acad Sci U S A 95(14): 832833.

Polak, J. M. and S. Van Noorden (2003). Applications of immunohistochemistry. Oxford, BIOS Scientific Publishers Ltd.

Pongs, O. (1992). Molecular biology of voltage-dependent potassium channels. Physiol Rev 72(4 Suppl): S69-88.

Portbury, A. L., J. B. Furness, H. M. Young, B. R. Southwell and S. R. Vigna (1996). Localisation of NK1 receptor immunoreactivity to neurons and interstitial cells of the guinea-pig gastrointestinal tract. J Comp Neurol 367(3): 342-51.

Powell, M., L. Prentice, T. Asawa, R. Kato, J. Sawicka, H. Tanaka, V. Petersen, A. Munkley, S. Morgan, B. Rees Smith and J. Furmaniak (1996). Glutamic acid decarboxylase autoantibody assay using 125I-labelled recombinant GAD65 produced in yeast. Clin Chim Acta 256(2): 175-88.

Preston, D. M. and J. E. Lennard-Jones (1986). Severe chronic constipation of young women: 'idiopathic slow transit constipation'. Gut 27(1): 41-8. 
Prior, C., B. Lang, D. Wray and J. Newsom-Davis (1985). Action of Lambert-Eaton myasthenic syndrome IgG at mouse motor nerve terminals. Ann Neurol 17(6): 587-92.

Rao, J. N., O. Platoshyn, L. Li, X. Guo, V. A. Golovina, J. X. Yuan and J. Y. Wang (2002). Activation of $\mathrm{K}(+)$ channels and increased migration of differentiated intestinal epithelial cells after wounding. Am J Physiol Cell Physiol 282(4): C885-98.

Rasmusson, R. L., M. J. Morales, R. C. Castellino, Y. Zhang, D. L. Campbell and H. C. Strauss (1995). C-type inactivation controls recovery in a fast inactivating cardiac $\mathrm{K}+$ channel (Kv1.4) expressed in Xenopus oocytes. J Physiol 489 ( Pt 3): 709-21.

Rees, W. D., R. J. Leigh, N. D. Christofides, S. R. Bloom and L. A. Turnberg (1982). Interdigestive motor activity in patients with systemic sclerosis. Gastroenterology 83(3): 575-80.

Ribeiro, U., Jr., A. V. Safatle-Ribeiro, A. Habr-Gama, J. J. Gama-Rodrigues, J. Sohn and J. C. Reynolds (1998). Effect of Chagas' disease on nitric oxide-containing neurons in severely affected and unaffected intestine. Dis Colon Rectum 41(11): 1411-7.

Richman, D. P., R. L. Wollmann, R. A. Maselli, C. M. Gomez, A. L. Corey, M. A. Agius and R. H. Fairclough (1993). Effector mechanisms of myasthenic antibodies. Ann N Y Acad Sci 681: 264-73.

Riemersma, S., A. Vincent, D. Beeson, C. Newland, S. Hawke, B. Vernet-der Garabedian, B. Eymard and J. Newsom-Davis (1996). Association of arthrogryposis multiplex congenita with maternal antibodies inhibiting fetal acetylcholine receptor function. J Clin Invest 98(10): 2358-63.

Rose, S., M. A. Young and J. C. Reynolds (1998). Gastrointestinal manifestations of scleroderma. Gastroenterol Clin North Am 27(3): 563-94.

Rueff, L., J. J. Graber, M. Bernbaum and R. I. Kuzniecky (2008). Voltage-gated potassium channel antibody-mediated syndromes: a spectrum of clinical manifestations. Rev Neurol Dis 5(2): 65-72.

Ruiz-de-Leon, A., J. Mendoza, C. Sevilla-Mantilla, A. M. Fernandez, J. Perez-de-laSerna, V. A. Gonzalez, E. Rey, A. Figueredo, M. Diaz-Rubio and E. G. De-la-Concha (2002). Myenteric antiplexus antibodies and class II HLA in achalasia. Dig Dis Sci 47(1): $15-9$.

Ruuska, T. H., R. Karikoski, V. V. Smith and P. J. Milla (2002). Acquired myopathic intestinal pseudo-obstruction may be due to autoimmune enteric leiomyositis. Gastroenterology 122(4): 1133-9.

Saiz, A., Y. Blanco, L. Sabater, F. Gonzalez, L. Bataller, R. Casamitjana, L. RamioTorrenta and F. Graus (2008). Spectrum of neurological syndromes associated with glutamic acid decarboxylase antibodies: diagnostic clues for this association. Brain 131(Pt 10): 2553-63.

Sato, S., I. Hayakawa, M. Hasegawa, M. Fujimoto and K. Takehara (2003). Function blocking autoantibodies against matrix metalloproteinase-1 in patients with systemic sclerosis. J Invest Dermatol 120(4): 542-7. 
Schaufele, N. and M. Diener (2005). Pharmacological characterisation of voltagedependent $\mathrm{Ca} 2+$ channels in isolated ganglia from the myenteric plexus. Life Sci 77(20): 2489-99.

Scheibner, J., A. U. Trendelenburg, L. Hein, K. Starke and C. Blandizzi (2002). Alpha 2adrenoceptors in the enteric nervous system: a study in alpha 2A-adrenoceptor-deficient mice. Br J Pharmacol 135(3): 697-704.

Schlaepfer, W. W. (1987). Neurofilaments: structure, metabolism and implications in disease. J Neuropathol Exp Neurol 46(2): 117-29.

Schobinger-Clement, S., H. A. Gerber and T. Stallmach (1999). Autoaggressive inflammation of the myenteric plexus resulting in intestinal pseudoobstruction. Am J Surg Pathol 23(5): 602-6.

Schuffler, M. D., H. W. Baird, C. R. Fleming, C. E. Bell, T. W. Bouldin, J. R. Malagelada, D. B. McGill, S. M. LeBauer, M. Abrams and J. Love (1983). Intestinal pseudo-obstruction as the presenting manifestation of small-cell carcinoma of the lung. A paraneoplastic neuropathy of the gastrointestinal tract. Ann Intern Med 98(2): 129-34.

Schuffler, M. D. and Z. Jonak (1982). Chronic idiopathic intestinal pseudo-obstruction caused by a degenerative disorder of the myenteric plexus: the use of Smith's method to define the neuropathology. Gastroenterology 82(3): 476-86.

Scott, V. E., Z. M. Muniz, S. Sewing, R. Lichtinghagen, D. N. Parcej, O. Pongs and J. O. Dolly (1994). Antibodies specific for distinct Kv subunits unveil a heterooligomeric basis for subtypes of alpha-dendrotoxin-sensitive $\mathrm{K}+$ channels in bovine brain. Biochemistry 33(7): 1617-23.

Seissler, J., J. Amann, L. Mauch, H. Haubruck, S. Wolfahrt, S. Bieg, W. Richter, R. Holl, E. Heinze, W. Northemann and et al. (1993). Prevalence of autoantibodies to the 65- and $67-\mathrm{kD}$ isoforms of glutamate decarboxylase in insulin-dependent diabetes mellitus. J Clin Invest 92(3): 1394-9.

Shellito, P. C. and A. L. Warshaw (1984). Idiopathic intermittent gastroparesis and its surgical alleviation. Am J Surg 148(3): 408-12.

Sheng, M., Y. J. Liao, Y. N. Jan and L. Y. Jan (1993). Presynaptic A-current based on heteromultimeric $\mathrm{K}+$ channels detected in vivo. Nature 365(6441): 72-5.

Shi, S. R., R. J. Cote and C. R. Taylor (2001). Antigen retrieval techniques: current perspectives. J Histochem Cytochem 49(8): 931-7.

Shillito, P., P. C. Molenaar, A. Vincent, K. Leys, W. Zheng, R. J. van den Berg, J. J. Plomp, G. T. van Kempen, G. Chauplannaz, A. R. Wintzen and et al. (1995). Acquired neuromyotonia: evidence for autoantibodies directed against $\mathrm{K}+$ channels of peripheral nerves. Ann Neurol 38(5): 714-22.

Sigala, S., G. Missale, C. Missale, V. Villanacci, R. Cestari, P. G. Grigolato, L. Lojacono and P. F. Spano (1995). Different neurotransmitter systems are involved in the development of esophageal achalasia. Life Sci 56(16): 1311-20. 
Smith, V. V., N. Gregson, L. Foggensteiner, G. Neale and P. J. Milla (1997). Acquired intestinal aganglionosis and circulating autoantibodies without neoplasia or other neural involvement. Gastroenterology 112(4): 1366-71.

Smith, V. V., B. D. Lake, M. A. Kamm and R. J. Nicholls (1992). Intestinal pseudoobstruction with deficient smooth muscle alpha-actin. Histopathology 21(6): 535-42.

Smith, V. V. and P. J. Milla (1997). Histological phenotypes of enteric smooth muscle disease causing functional intestinal obstruction in childhood. Histopathology 31(2): 11222.

Snyders, D. J., M. M. Tamkun and P. B. Bennett (1993). A rapidly activating and slowly inactivating potassium channel cloned from human heart. Functional analysis after stable mammalian cell culture expression. J Gen Physiol 101(4): 513-43.

Sodhi, N., M. Camilleri, J. K. Camoriano, P. A. Low, R. D. Fealey and M. C. Perry (1989). Autonomic function and motility in intestinal pseudoobstruction caused by paraneoplastic syndrome. Dig Dis Sci 34(12): 1937-42.

Sonoda, Y., K. Arimura, A. Kurono, M. Suehara, M. Kameyama, S. Minato, A. Hayashi and M. Osame (1996). Serum of Isaacs' syndrome suppresses potassium channels in PC12 cell lines. Muscle Nerve 19(11): 1439-46.

Spiller, R. C., D. Jenkins, J. P. Thornley, J. M. Hebden, T. Wright, M. Skinner and K. R. Neal (2000). Increased rectal mucosal enteroendocrine cells, $\mathrm{T}$ lymphocytes, and increased gut permeability following acute Campylobacter enteritis and in postdysenteric irritable bowel syndrome. Gut 47(6): 804-11.

Stanghellini, V., M. Camilleri and J. R. Malagelada (1987). Chronic idiopathic intestinal pseudo-obstruction: clinical and intestinal manometric findings. Gut 28(1): 5-12.

Stanley, E. F. and D. B. Drachman (1978). Effect of myasthenic immunoglobulin on acetylcholine receptors of intact mammalian neuromuscular junctions. Science 200(4347): 1285-7.

Starodub, A. M. and J. D. Wood (1999). Selectivity of omega-CgTx-MVIIC toxin from Conus magus on calcium currents in enteric neurons. Life Sci 64(26): PL305-10.

Stefaneanu, L., N. Ryan and K. Kovacs (1988). Immunocytochemical localization of synaptophysin in human hypophyses and pituitary adenomas. Arch Pathol Lab Med 112(8): 801-4.

Sterin-Borda, L., J. C. Goin, C. R. Bilder, G. Iantorno, A. C. Hernando and E. Borda (2001). Interaction of human chagasic IgG with human colon muscarinic acetylcholine receptor: molecular and functional evidence. Gut 49(5): 699-705.

Sterin-Borda, L., C. P. Leiros, J. C. Goin, G. Cremaschi, A. Genaro, A. V. Echague and E. Borda (1997). Participation of nitric oxide signaling system in the cardiac muscarinic cholinergic effect of human chagasic IgG. J Mol Cell Cardiol 29(7): 1851-65.

Stolinski, C., A. S. Breathnach, B. Martin, P. K. Thomas, R. H. King and G. Gabriel (1981). Associated particle aggregates in juxtaparanodal axolemma and adaxonal Schwann cell membrane of rat peripheral nerve. J Neurocytol 10(4): 679-91. 
Storch, W. B., V. F. Eckardt, M. Wienbeck, T. Eberl, P. G. Auer, A. Hecker, T. Junginger and H. Bosseckert (1995). Autoantibodies to Auerbach's plexus in achalasia. Cell Mol Biol (Noisy-le-grand) 41(8): 1033-8.

Stuhmer, W., J. P. Ruppersberg, K. H. Schroter, B. Sakmann, M. Stocker, K. P. Giese, A. Perschke, A. Baumann and O. Pongs (1989). Molecular basis of functional diversity of voltage-gated potassium channels in mammalian brain. EMBO J 8(11): 3235-44.

Suarez-Kurtz, G., R. Vianna-Jorge, B. F. Pereira, M. L. Garcia and G. J. Kaczorowski (1999). Peptidyl inhibitors of shaker-type Kv1 channels elicit twitches in guinea pig ileum by blocking kv1.1 at enteric nervous system and enhancing acetylcholine release. J Pharmacol Exp Ther 289(3): 1517-22.

Tan, F. K., F. C. Arnett, J. D. Reveille, C. Ahn, S. Antohi, T. Sasaki, K. Nishioka and C. A. Bona (2000). Autoantibodies to fibrillin 1 in systemic sclerosis: ethnic differences in antigen recognition and lack of correlation with specific clinical features or HLA alleles. Arthritis Rheum 43(11): 2464-71.

Terwindt, G. M., R. A. Ophoff, J. Haan, L. A. Sandkuijl, R. R. Frants and M. D. Ferrari (1998). Migraine, ataxia and epilepsy: a challenging spectrum of genetically determined calcium channelopathies. Dutch Migraine Genetics Research Group. Eur J Hum Genet 6(4): 297-307.

Thornbury, K. D., S. M. Ward and K. M. Sanders (1992). Outward currents in longitudinal colonic muscle cells contribute to spiking electrical behavior. Am J Physiol 263(1 Pt 1): C237-45.

Thornbury, K. D., S. M. Ward and K. M. Sanders (1992). Participation of fast-activating, voltage-dependent $\mathrm{K}$ currents in electrical slow waves of colonic circular muscle. Am J Physiol 263(1 Pt 1): C226-36.

Tomimitsu, H., K. Arimura, T. Nagado, O. Watanabe, R. Otsuka, A. Kurono, Y. Sonoda, M. Osame and M. Kameyama (2004). Mechanism of action of voltage-gated $\mathrm{K}+$ channel antibodies in acquired neuromyotonia. Ann Neurol 56(3): 440-4.

Tornblom, H., H. Abrahamsson, G. Barbara, P. M. Hellstrom, G. Lindberg, H. Nyhlin, B. Ohlsson, M. Simren, K. Sjolund, H. Sjovall, P. T. Schmidt and L. Ohman (2005). Inflammation as a cause of functional bowel disorders. Scand J Gastroenterol 40(10): 1140-8.

Tornblom, H., B. Lang, L. Clover, C. H. Knowles, A. Vincent and G. Lindberg (2007). Autoantibodies in patients with gut motility disorders and enteric neuropathy. Scand $\mathrm{J}$ Gastroenterol 42(11): 1289-93.

Tornblom, H., G. Lindberg, B. Nyberg and B. Veress (2002). Full-thickness biopsy of the jejunum reveals inflammation and enteric neuropathy in irritable bowel syndrome. Gastroenterology 123(6): 1972-9.

Toyka, K. V., D. B. Drachman, D. E. Griffin, A. Pestronk, J. A. Winkelstein, K. H. Fishbeck and I. Kao (1977). Myasthenia gravis. Study of humoral immune mechanisms by passive transfer to mice. N Engl J Med 296(3): 125-31. 
Tozzoli, R. (2007). Recent advances in diagnostic technologies and their impact in autoimmune diseases. Autoimmun Rev 6(6): 334-40.

Treacy, W. L., A. H. Baggenstoss, C. H. Slocumb and C. F. Code (1963). Scleroderma of the oesophagus. A correlation of histologic and physiologic findings. Ann Intern Med 59: 351-6.

Valenzuela, D. M., T. N. Stitt, P. S. DiStefano, E. Rojas, K. Mattsson, D. L. Compton, L. Nunez, J. S. Park, J. L. Stark, D. R. Gies and et al. (1995). Receptor tyrosine kinase specific for the skeletal muscle lineage: expression in embryonic muscle, at the neuromuscular junction, and after injury. Neuron 15(3): 573-84.

van Vuurden, D. G., F. B. Plotz, M. de Jong, A. Bokenkamp and J. A. van Wijk (2005). Therapeutic total plasma exchange in a child with neuroblastoma-related anti-Hu syndrome. Pediatr Nephrol 20(11): 1655-6.

Van Wagoner, D. R., A. L. Pond, P. M. McCarthy, J. S. Trimmer and J. M. Nerbonne (1997). Outward $\mathrm{K}+$ current densities and Kv1.5 expression are reduced in chronic human atrial fibrillation. Circ Res 80(6): 772-81.

Veh, R. W., R. Lichtinghagen, S. Sewing, F. Wunder, I. M. Grumbach and O. Pongs (1995). Immunohistochemical localization of five members of the Kv1 channel subunits: contrasting subcellular locations and neuron-specific co-localizations in rat brain. Eur J Neurosci 7(11): 2189-205.

Verne, G. N., A. B. Hahn, B. C. Pineau, B. J. Hoffman, B. W. Wojciechowski and W. C. $\mathrm{Wu}$ (1999). Association of HLA-DR and -DQ alleles with idiopathic achalasia. Gastroenterology 117(1): 26-31.

Verne, G. N., J. E. Sallustio and E. Y. Eaker (1997). Anti-myenteric neuronal antibodies in patients with achalasia. A prospective study. Dig Dis Sci 42(2): 307-13.

Vernino, S., J. Adamski, T. J. Kryzer, R. D. Fealey and V. A. Lennon (1998). Neuronal nicotinic ACh receptor antibody in subacute autonomic neuropathy and cancer-related syndromes. Neurology 50(6): 1806-13.

Vernino, S., L. G. Ermilov, L. Sha, J. H. Szurszewski, P. A. Low and V. A. Lennon (2004). Passive transfer of autoimmune autonomic neuropathy to mice. J Neurosci 24(32): 7037-42.

Vernino, S., P. A. Low, R. D. Fealey, J. D. Stewart, G. Farrugia and V. A. Lennon (2000). Autoantibodies to ganglionic acetylcholine receptors in autoimmune autonomic neuropathies. N Engl J Med 343(12): 847-55.

Vernino, S., P. A. Low and V. A. Lennon (2003). Experimental autoimmune autonomic neuropathy. J Neurophysiol 90(3): 2053-9.

Viallard, J. F., A. Vincent, J. F. Moreau, M. Parrens, J. L. Pellegrin and E. Ellie (2005). Thymoma-associated neuromyotonia with antibodies against voltage-gated potassium channels presenting as chronic intestinal pseudo-obstruction. Eur Neurol 53(2): 60-3.

Vianna-Jorge, R., C. F. Oliveira, M. L. Garcia, G. J. Kaczorowski and G. Suarez-Kurtz (2000). Correolide, a nor-triterpenoid blocker of Shaker-type Kv1 channels elicits 
twitches in guinea-pig ileum by stimulating the enteric nervous system and enhancing neurotransmitter release. Br J Pharmacol 131(4): 772-8.

Viglione, M. P., T. J. O'Shaughnessy and Y. I. Kim (1995). Inhibition of calcium currents and exocytosis by Lambert-Eaton syndrome antibodies in human lung cancer cells. J Physiol 488 ( Pt 2): 303-17.

Vincent, A. (2002). Measuring and evaluating the significance of autoantibodies in neurological disorders. Clinical and Applied Immunology Reviews 3(3): 127-151.

Vincent, A., S. R. Irani and B. Lang (2010). The growing recognition of immunotherapyresponsive seizure disorders with autoantibodies to specific neuronal proteins. Curr Opin Neurol 23(2): 144-50.

Vincent, A., B. Lang and K. A. Kleopa (2006). Autoimmune channelopathies and related neurological disorders. Neuron 52(1): 123-38.

Vincent, A. and J. Newsom-Davis (1985). Acetylcholine receptor antibody as a diagnostic test for myasthenia gravis: results in 153 validated cases and 2967 diagnostic assays. J Neurol Neurosurg Psychiatry 48(12): 1246-52.

Vincent, A. and N. Willcox (1999). The role of T-cells in the initiation of autoantibody responses in thymoma patients. Pathol Res Pract 195(8): 535-40.

Wade, G. R., L. G. Laurier, H. G. Preiksaitis and S. M. Sims (1999). Delayed rectifier and $\mathrm{Ca}(2+)$-dependent $\mathrm{K}(+)$ currents in human esophagus: roles in regulating muscle contraction. Am J Physiol 277(4 Pt 1): G885-95.

Wang, H., D. D. Kunkel, T. M. Martin, P. A. Schwartzkroin and B. L. Tempel (1993). Heteromultimeric $\mathrm{K}+$ channels in terminal and juxtaparanodal regions of neurons. Nature 365(6441): 75-9.

Wang, H., D. D. Kunkel, P. A. Schwartzkroin and B. L. Tempel (1994). Localization of Kv1.1 and Kv1.2, two K channel proteins, to synaptic terminals, somata, and dendrites in the mouse brain. J Neurosci 14(8): 4588-99.

Wang, L. H., X. C. Fang and G. Z. Pan (2004). Bacillary dysentery as a causative factor of irritable bowel syndrome and its pathogenesis. Gut 53(8): 1096-101.

Wang, Z., B. Fermini and S. Nattel (1993). Sustained depolarization-induced outward current in human atrial myocytes. Evidence for a novel delayed rectifier $\mathrm{K}+$ current similar to Kv1.5 cloned channel currents. Circ Res 73(6): 1061-76.

Waterman, S. A., B. Lang and J. Newsom-Davis (1997). Effect of Lambert-Eaton myasthenic syndrome antibodies on autonomic neurons in the mouse. Ann Neurol 42(2): 147-56.

Werth, B., B. Meyer-Wyss, G. A. Spinas, J. Drewe and C. Beglinger (1992). Noninvasive assessment of gastrointestinal motility disorders in diabetic patients with and without cardiovascular signs of autonomic neuropathy. Gut 33(9): 1199-203.

Wiedenmann, B. and W. W. Franke (1985). Identification and localization of synaptophysin, an integral membrane glycoprotein of $\mathrm{Mr} 38,000$ characteristic of presynaptic vesicles. Cell 41(3): 1017-28. 
Wiedenmann, B., W. W. Franke, C. Kuhn, R. Moll and V. E. Gould (1986). Synaptophysin: a marker protein for neuroendocrine cells and neoplasms. Proc Natl Acad Sci U S A 83(10): 3500-4.

Willis, T. (1674). Pharmaceutice Rationalis Sive Diatribe Medicamentorum Operationibus In Human Corpore. Hagae Comitis, London.

Willison, H. J. and N. Yuki (2002). Peripheral neuropathies and anti-glycolipid antibodies. Brain 125(Pt 12): 2591-625.

Wingate, D., M. Hongo, J. Kellow, G. Lindberg and A. Smout (2002). Disorders of gastrointestinal motility: towards a new classification. J Gastroenterol Hepatol 17 Suppl: S1-14.

Wolfe, G. I. and S. J. Oh (2008). Clinical phenotype of muscle-specific tyrosine kinaseantibody-positive myasthenia gravis. Ann N Y Acad Sci 1132: 71-5.

Yoshimura, N. and W. C. De Groat (1996). Characterization of voltage-sensitive $\mathrm{Na}+$ and $\mathrm{K}+$ currents recorded from acutely dissociated pelvic ganglion neurons of the adult rat. $\mathrm{J}$ Neurophysiol 76(4): 2508-21.

Yoshimura, N., G. White, F. F. Weight and W. C. de Groat (1996). Different types of $\mathrm{Na}+$ and A-type $\mathrm{K}+$ currents in dorsal root ganglion neurones innervating the rat urinary bladder. J Physiol 494 ( Pt 1): 1-16.

Zarate, N., X. Y. Wang, G. Tougas, M. Anvari, D. Birch, F. Mearin, J. R. Malagelada and J. D. Huizinga (2006). Intramuscular interstitial cells of Cajal associated with mast cells survive nitrergic nerves in achalasia. Neurogastroenterol Motil 18(7): 556-68.

Zhang, L., D. Hu, J. Li, Y. Wu, X. Liu and X. Yang (2002). Autoantibodies against the myocardial betal-adrenergic and M2-muscarinic receptors in patients with congestive heart failure. Chin Med J (Engl) 115(8): 1127-31.

Zholos, A. V., L. V. Baidan, A. M. Starodub and J. D. Wood (1999). Potassium channels of myenteric neurons in guinea-pig small intestine. Neuroscience 89(2): 603-18. 